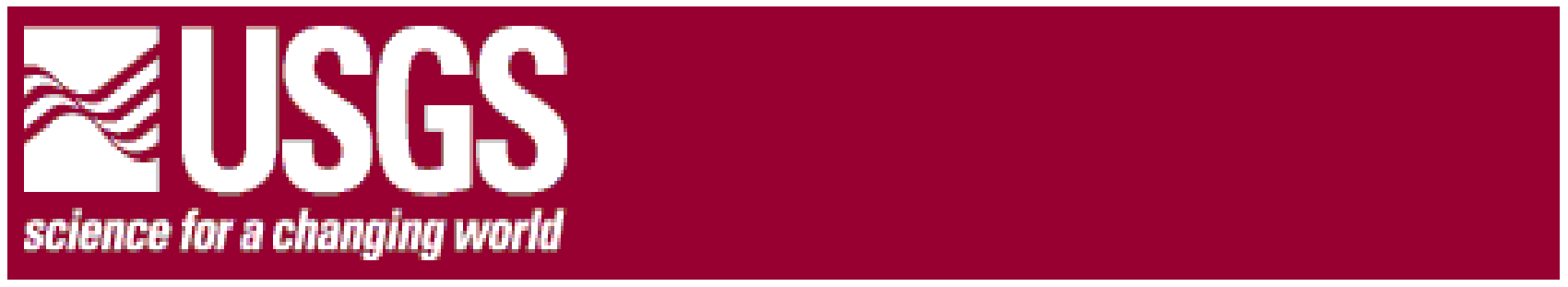

\title{
Hawaiian Volcano Observatory Summary 100; Part I, Seismic Data, January to December 2000
}

by Jennifer S. Nakata

Chronological Summary

by C. Heliker \& D. Sherrod

Open-File Report 01-332

2001

This report is preliminary and has not been reviewed for conformity with U.S. Geological Survey editorial standards or with the North American Stratigraphic Code. Any use of trade, product, or firm names is for descriptive purposes only and does not imply endorsement by the U.S. Government.

\section{U.S. DEPARTMENT OF THE INTERIOR}

\section{U.S. GEOLOGICAL SURVEY}

Hawaiian Volcano Observatory

Hawai'i Volcanoes National Park, Hawai'i 96718 
Hawaiian Volcano Observatory Staff .....................................................................................

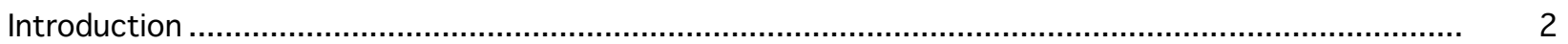

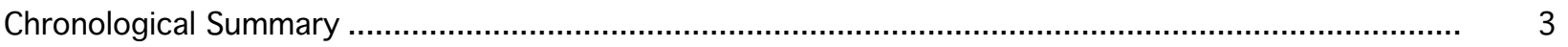

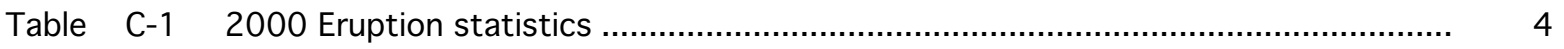

Table C-2 Episode 55 pauses, surges and intrusions ........................................................ 5

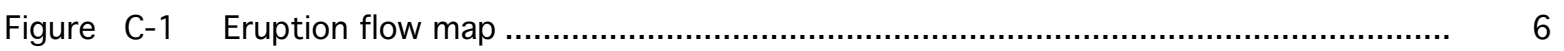

Figure C-2 Map of Pu'u 'O`o features .............................................................................. 7

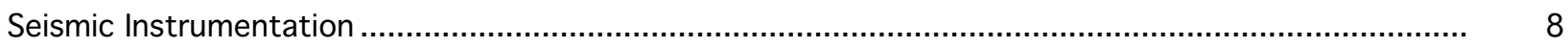

Figure 1 Map of Hawai'i Island showing geographic and geologic features ........................... 9

Figure 2 Seismic stations operated by the USGS and NOAA on Hawai'i Island ....................... 10

Figure 3 Seismic network telemetry scheme on Hawaiłi Island .......................................... 11

Figure $\quad 4 a \quad$ Seismic network telemetry scheme at Kilauea summit ........................................ 12

Figure $\quad 4 b$ Broad-band telemetry scheme at Kilauea summit ............................................. 12

Figure 5 Seismic network telemetry scheme on Maui Island ........................................... 13

Table 1 Seismic stations in Hawai'i operated by the USGS .............................................. 14

Table $\quad 2$ Seismic instrument types in use by HVO ...................................................... 16

Figure $\quad 6 \quad$ HVO system response curve of the four basic seismograph types ........................ 16

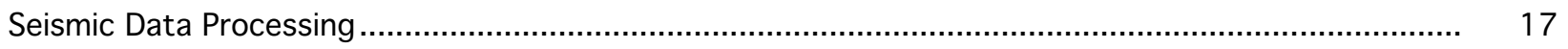

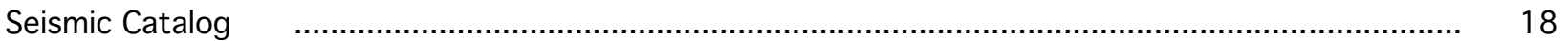

Table $\quad 3$ Coordinates of named regions used for classifying earthquakes ............................ 18

Figure $\quad 7$ Earthquake classification, shallow for Kilauea and Mauna Loa ................................ 20

Figure $\quad 8$ Earthquake classification, intermediate for Kilauea and Mauna Loa ........................ 21

Figure 9 Earthquake classification, crustal, for Hawai'i Island .......................................... 22

Figure 10 Earthquake classification, deep, for Hawai'i Island ............................................... 23

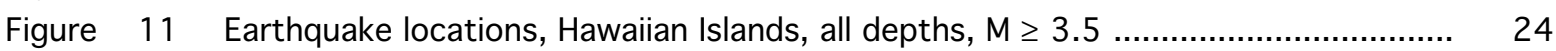

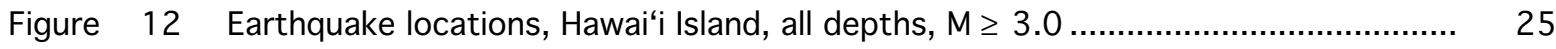

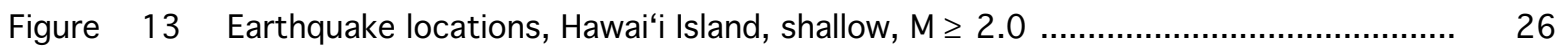

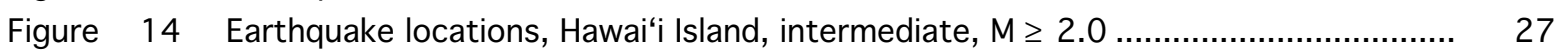

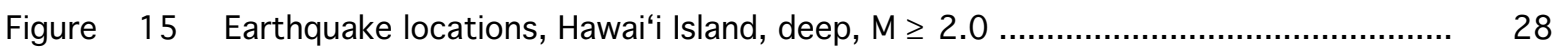

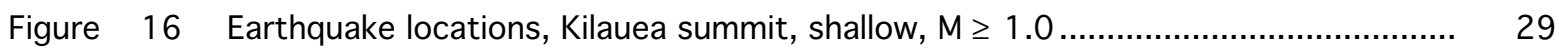

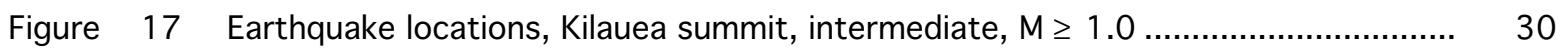

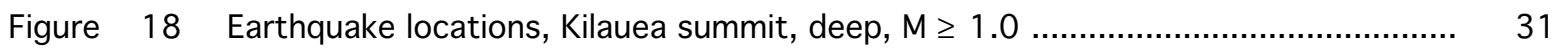

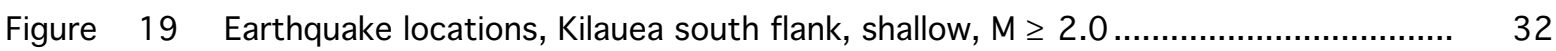

Figure 20 Earthquake locations, Kilauea south flank, intermediate, $M \geq 2.0 \ldots \ldots \ldots \ldots \ldots \ldots \ldots \ldots . . . . . . . . . . . . . . .33$

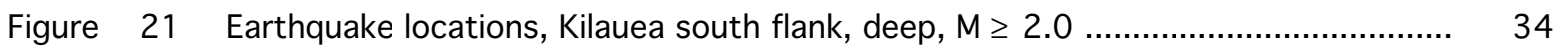

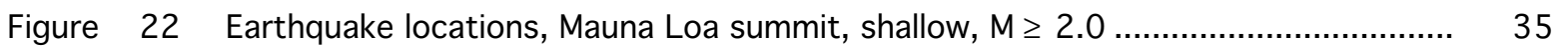

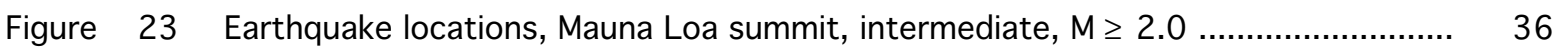

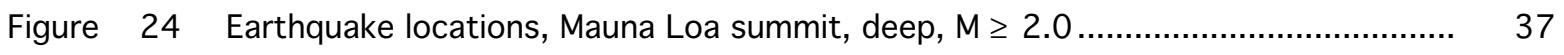

Table $\quad 4 \quad$ List of all located earthquakes ....................................................................... 38

Table 5 List of located earthquakes of magnitude 3.0 or greater .................................... 75 


\section{HAWAIIAN VOLCANO OBSERVATORY STAFF}

DONALD A. SWANSON (SCIENTIST-IN-CHARGE)

ARNOLD T. OKAMURA (DEPUTY SCIENTIST-IN-CHARGE)

\section{GEOLOGY}

C. CHRISTINA HELIKER

DAVID SHERROD

FRANK A. TRUSDELL

GEOPHYSICS

JAMES P. KAUAHIKAUA

SEISMOLOGY

STUART K. KOYANAGI

JENNIFER S. NAKATA

PAUL G. OKUBO

ALVIN H. TOMORI

DEFORMATION

MICHAEL LISOWSKI*

ASTA MIKLIUS

MAURICE K. SAKO

GEOCHEMISTRY

TAMAR ELIAS

A. JEFFERSON SUTTON

ELECTRONICS

STEVEN FUKE

BRUCE FURUKAWA

KENNETH T. HONMA

\section{COMPUTER}

WILFRED R. TANIGAWA

\section{LIBRARY/PHOTO ARCHIVE}

T. JANE TAKAHASHI

ADMINISTRATION

PAULINE N. FUKUNAGA

MARIAN M. KAGIMOTO

PROGRAM OUTREACH COORDINATOR

STEVE BRANTLEY

SCIENTIST EMERITUS

DALLAS B. JACKSON

ROBERT Y. KOYANAGI

\section{CONTRACTS}

Seismic Record Changing

L. GLADYS FORBES

ADOLPH R. TEVES

* Left during 2000 


\section{INTRODUCTION}

The Hawaiian Volcano Observatory (HVO) summary presents seismic data gathered during the year and a chronological narrative describing the volcanic events. The seismic summary is offered without interpretation as a source of preliminary data. It is complete in the sense that all data for events of $M \geq 1.5$ routinely gathered by the Observatory are included. The emphasis in collection of tilt and deformation data has shifted from quarterly measurements at a few water-tube tilt stations ("wet" tilt) to a larger number of continuously recording borehole tiltmeters, repeated measurements at numerous spirit-level tilt stations ("dry" tilt), and surveying of level and trilateration networks. Because of the large quantity of deformation data now gathered and differing schedules of data reduction, the seismic and deformation summaries are published separately.

The HVO summaries have been published in various forms since 1956. Summaries prior to 1974 were issued quarterly, but cost, convenience of preparation and distribution, and the large quantities of data dictated an annual publication beginning with Summary 74 for the year 1974 . Summary 86 (the introduction of CUSP at HVO) includes a description of the seismic instrumentation, calibration, and processing used in recent years. The present summary includes enough background information on the seismic network and processing to allow use of the data and to provide an understanding of how they were gathered.

A report tabulating instrumentation, calibration, and recording history of each seismic station in the network by Klein and Koyanagi is available as a USGS Open-File Report ${ }^{1}$. It is designed as a reference for users of seismograms and phase data and includes and augments the information in the station table in this summary.

1 Klein, F.W., and Koyanagi, R.Y., 1980, Hawaiian Volcano Observatory seismic network history, 1950-1979: U.S. Geological Survey Open-File Report 80-302, 84 p. 


\section{CHRONOLOGICAL SUMMARY 2000 \\ by \\ C. Heliker and D. Sherrod}

Until last spring, no houses had been overrun since late 1991. But in the last nine months, three longabandoned structures in lower Royal Gardens were destroyed by the rising tide of pahoehoe on the coastal plain. Flows covered $6 \mathrm{~km}^{2}$ in 2000 (fig. C-1), but only $2 \mathrm{~km}^{2}$ of that was virgin, vegetated land, the rest being previously covered by lava during this eruption. The total area covered by lava since 1983 is $104 \mathrm{~km}^{2}$ $\left(40 \mathrm{mi}^{2}\right)$, and the volume of lava has topped $2 \mathrm{~km}^{3}\left(0.5 \mathrm{mi}^{3}\right)$. For the latest statistics, refer to table $\mathrm{C}-1$.

The magma supply to the Pu'u 'O'o flank vent(s) that feed the tube system paused briefly twice in 2000 (table C-2). The frequency of pauses has dropped considerably since the beginning of episode 55 , but their complexity has increased. In part, this is due to a surge in the amount of data each pause generates, now that borehole tiltmeters have been installed from the summit down the east rift zone to Pu'u 'O'o cone. But even without considering the new tilt data, it is obvious that the role of the summit in the pauses has become much more variable, both in terms of tilt and seismicity. As episode 55 progresses, it is increasingly difficult to characterize a "typical" pause.

In addition to two pauses, 2000 brought one upper east rift zone intrusion (February 23) and one magmatic surge from the summit to Pu'u 'O'o (September 24). Neither of these events was followed by a bona fide pause, unlike the previous intrusion and surge in episode 55 (September 1999 and January 1998, respectively). There is some evidence that the February 2000 intrusion caused the eruption to pause or slow down for less than $4 \mathrm{hrs}$, but it is impossible to know for sure since the event was so brief. In contrast, the intrusion of September 1999 depressurized the dike feeding the eruption, causing part of the Pu'u 'O'o crater floor to collapse and the eruption to pause.

The Dog Day surge in September was the first such event of this magnitude since the January 14 surge in 1998. Unlike the earlier event, however, the Dog Day surge was not followed by a pause.

The ocean entries spanned more of the coastline in 2000 than in many years past, extending $5 \mathrm{~km}$ from Lae'apuki to the east edge of the episode 55 flow field. At the beginning of the year, the only ocean entry was at Lae'apuki, but in February the broad front of the "Smoke" flow reached the coast and, over the next five months, coated the entire sea cliff from Kamokuna to the east edge of the flow field-bad news if you were a seabird (and there were many) nesting along that particular stretch of coastline.

The main ocean entry was at Waha'ula beginning in April, but smaller entries persisted to the east. When lava reached the ocean again, following pause 30 in late August, Kamokuna became the sole entry. About 7.5 hectares (18.5 acres) of new land were added to the island in 2000. The largest bench collapse of the year, on November 16, was witnessed by a pilot who saw $\sim 1$ ha of bench slide into the sea within a few seconds.

The crater of Pu'u 'O'o changed very little this year (fig. C-2). Since the brief period of lava pond activity in September-October 1999, only a few small pads of pahoehoe have extruded on the crater floor. There was some subsidence of the drained pond bed or "trough" near vents, and the "July pit,", which formed in late July, has since formed a spatter cone. At the end of the year, the crater floor inside the "trough" (the empty pond left over from late 1999) near the July pit was about $67 \mathrm{~m}$ below our camera site on the north rim (or $\sim 51 \mathrm{~m}$ below the low point on the east rim, which is our traditional datum for crater depths). The terrace that encircles the trough was about $50 \mathrm{~m}(34 \mathrm{~m})$ below the camera site, as compared with about $55 \mathrm{~m} \mathrm{(39} \mathrm{m)} \mathrm{at}$ the end of last year. These measurements were made by a reflectorless laser range-finder.

Outside the crater, the west gap pit wins the "most changed" prize for 2000 . The spatter cone on the southwest side of the cone collapsed in July, leaving an inner crater about $20 \mathrm{~m}$ deep that emitted sloshing sounds when first formed. This collapse pit, which was soon quiet and floored with talus, continued to expand for the next few weeks, slightly enlarging the main pit. During the Dog Day surge, active lava covered the bottom of the west gap pit for $8 \mathrm{hrs}$ before it drained, leaving the pit 35-40 m deep.

Puka Nui, the very large collapse pit on the southwest flank of Pu'u 'O'o, showed little change in size this year. A small inner collapse pit, first observed in July, claimed one of the spatter cones that formed inside Puka Nui during 1999. Mass wasting continued to eat away at ridge separating Puka Nui from the crater.

Lua Hou, a small pit on the shield just south of Puka Nui, was first observed in February. Flowing lava was observed at the bottom of it following the February intrusion. The pit was floored by ponded lava, or at least was very close to active, through September. The next time it was observed from the air, in January 2001, it was dark. 
Table C-1. Eruption Statistics

Areas

$\overline{\text { Total }}$ area covered by lava, $3 / 83$ - 12/31/00: $\mathbf{1 0 3 . 7} \mathbf{k m}^{2}\left(40 \mathrm{mi}^{2}\right.$ ) Episode Area originally covered

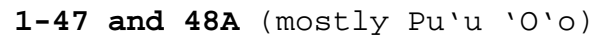

48 (Kupaianaha)

$42.0 \mathrm{~km}^{2}$

49 (between $\mathrm{Pu}$ 'u 'O'O and Kupaianaha)

41.0

3.9

50-53 (Pu'u 'O'O flank vents)

54 (in and $\mathrm{NE}$ of Napau Crater)

26.4

0.24

55 (Pu'u 'O'O flank vents) 32.3

New (vegetated) territory covered in 2000: $2.2 \mathrm{~km}^{2}$

Net total of new land created, Nov 86 - Dec 2000: 207 hectares (510 acres)\#

Net new land created during 2000: 7.5 hectares (18.5 acres)

\#These figures do not include new land that was claimed by wave erosion or collapse of the active lava bench. Due to these processes, mapping in 1998 and 1999 revealed a decrease in total acreage.

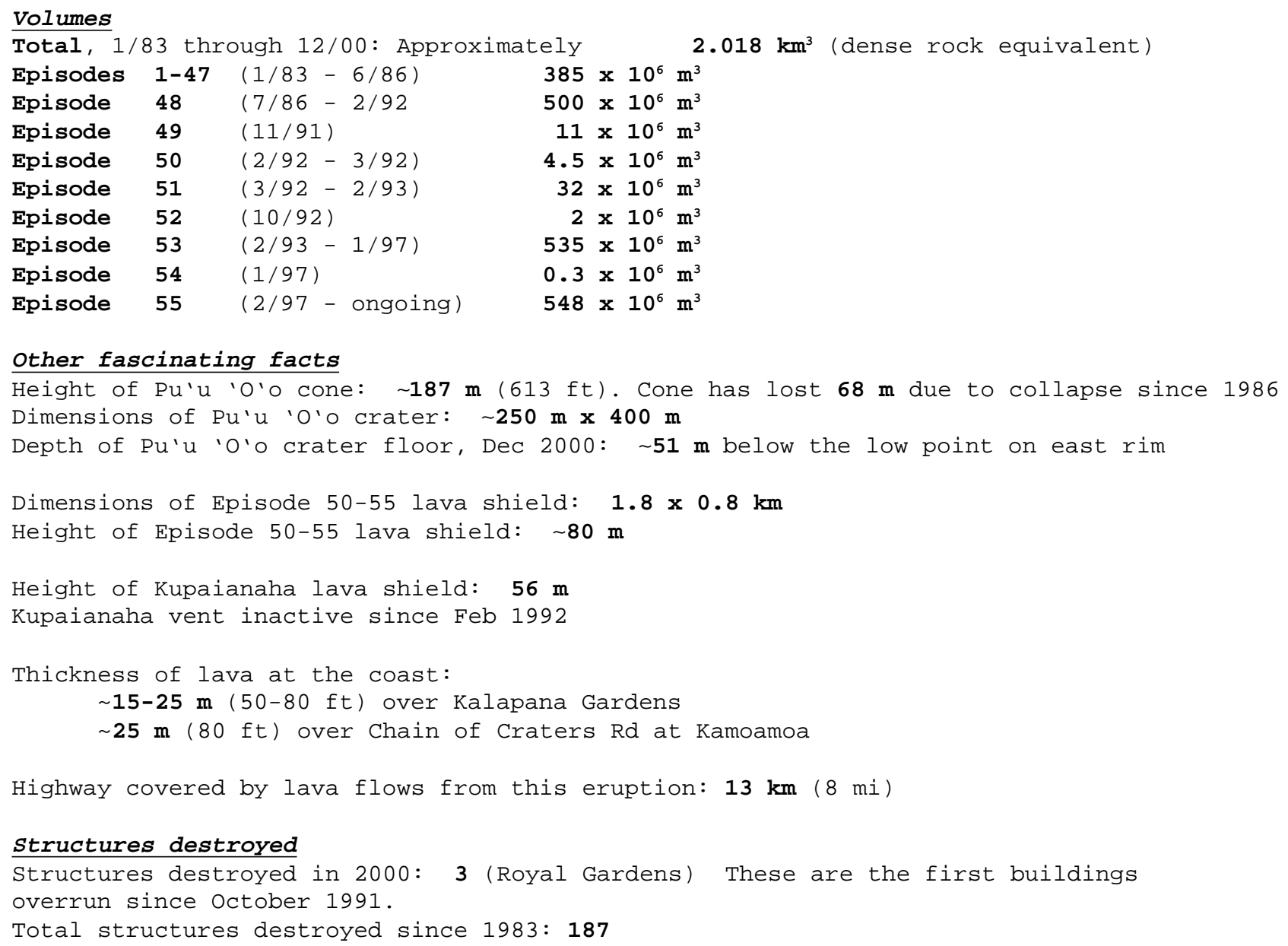



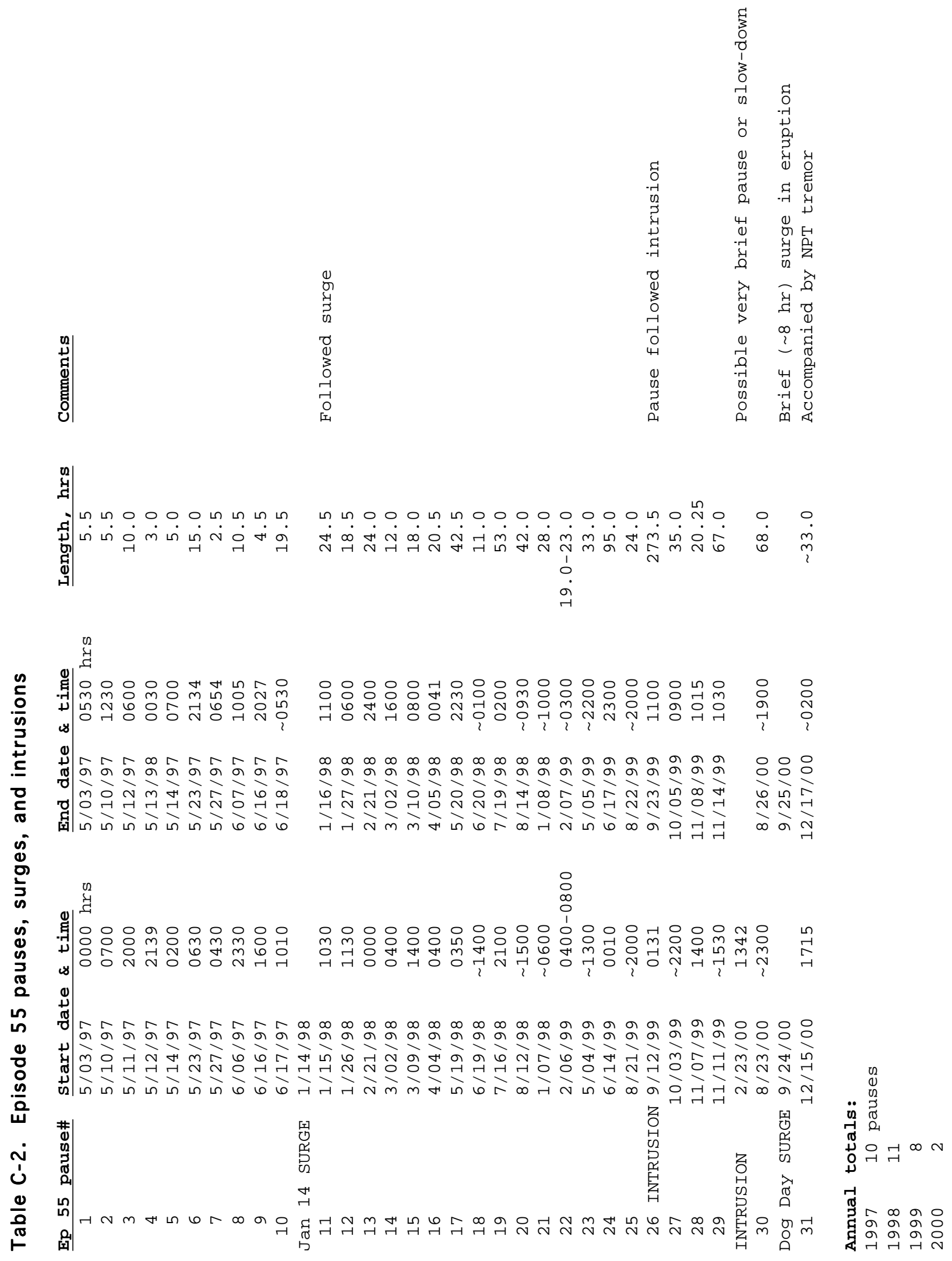


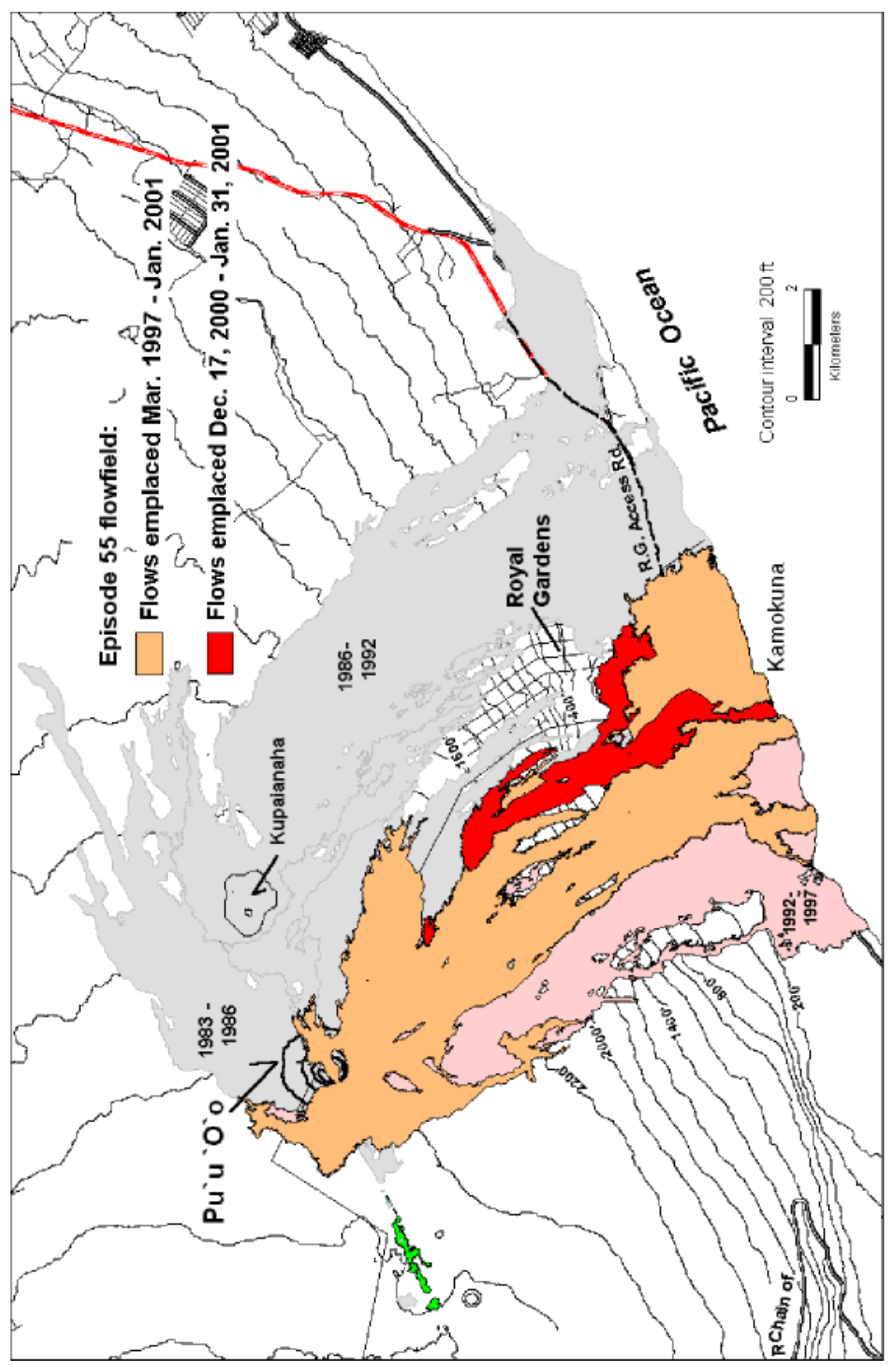

Figure C-1. The eruption site, showing flows emplaced from October 6, 1999 (end of pause 27) through 2000. 


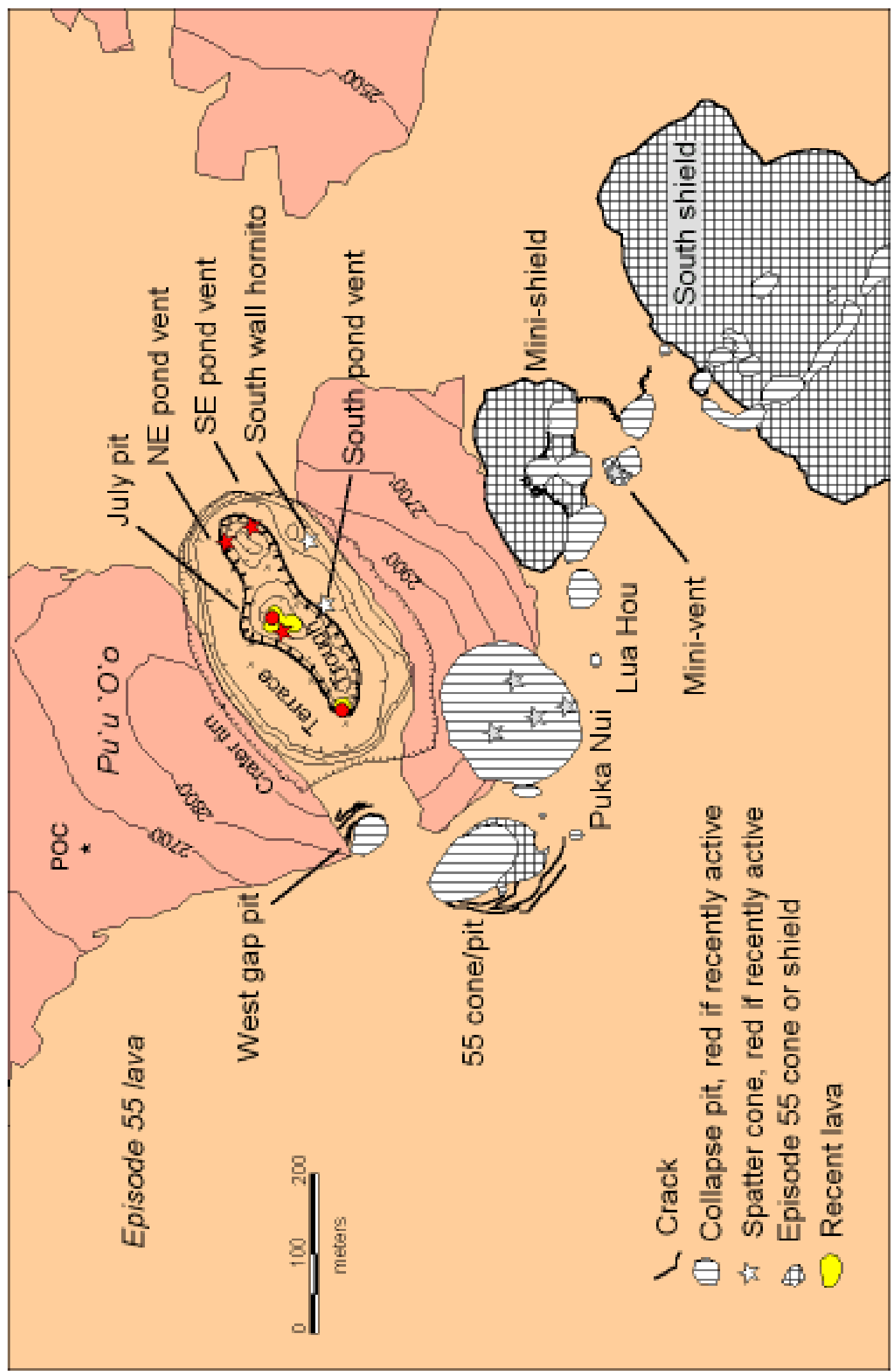

Figure C-2. Map of Pu'u 'O'o showing various features inside the crater and on the flanks of the cone as of early January 2001. 


\section{SEISMIC INSTRUMENTATION}

The network. The Hawaiian Volcano Observatory maintains an extensive telemetered seismic network on the Island of Hawai' $\mathrm{i}$. The standard HVO field sensors, $1-\mathrm{Hz}$ geophones, are deployed as single-component, vertical-only units or as three-component combinations of one vertical and two orthogonal horizontal units. The 2000 network consisted of 50 station sites: 10 three-component, 2 six-component (which included a three-component Kinemetric Force-Balance accelerometer), one four-component (which included a low-gain vertical with a unity gain setting), one four-component and two two-component (each site included a moderate-gain vertical with a $48 \mathrm{db}$ setting), and 34 vertical-component-only sites. The coverage is most dense on and around Kilauea Volcano. During 1999 HVO added to the network three vertical-component-only sites on the Island of Maui. All seismic signals from the network are telemetered in real time to the Observatory for recording.

The Pacific Isunami Warning Center (NOAA) operates and maintains a network of stations on the islands of Hawai'i, Maui, and O'ahu. In 1999, radio links were established to share data, in real-time, between PTWC and HVO. PTWC signals from one O'ahu three-component station, and one Maui and four Hawai'i verticalcomponent-only stations, were telemetered to the Observatory for recording.

Figure 1 is a map of selected geographic and geologic features. Figure 2 shows the seismic stations sites operated by HVO and PTWC on the Island of Hawai'i during 2000. Figure 3 indicates the telemetry scheme for the seismic stations on Hawai $i$ Island, and figures $4 a$ and $4 b$ are expanded views of the telemetry schemes at Kilauea summit: 4a, HVO seismic stations and 4b, broadband network installed by Menlo Park and maintained by HVO. Figure 5 indicates the telemetry scheme for the seismic stations on Maui Island.

Table 1 lists seismic stations by names, four-letter station codes, coordinates in degrees and minutes, elevation in meters, and other data, as described below, pertaining to each station. The list includes all the stations operated by HVO during 2000. Seismic stations operated by PTWC on the Islands of Hawai'i, O'ahu and Maui are also listed. Phase times from PTWC stations, not telemetered to HVO, are used to supplement local earthquakes and earthquakes that occur within the Hawaiian Archipelago but distant from the Hawai' $i$ Island network.

Instrumentation and recording. Each telemetered station's data channel has a voltage-controlled oscillator (VCO) for FM multiplex transmission to HVO via radio. These telemetering stations are all of Type 1, Earthquake Hazards Team (EHT) standard system used in USGS seismic networks (see table 2 for details). After discrimination at the receiver, the analog signals are converted to digital form as part of the routine computer location processing and archiving. Continuous signals from the telemetered network are saved on 4-mm digital-audio tape (DAT) recording units. Three DAT recorders run in automatic rotation, as each 20-hr tape is filled. Optic recordings are coded in table 1 as follows: $\mathrm{H}$ - Helicorder paper, and I - ink paper. DAT and paper records are archived at HVO.

Seismograph response and calibration. Displacement response curve for the short-period seismograph type in use is given in figure 6. The Type 1 curve gives the displacement magnification of the standard EHT system from ground motion at the seismometer to the seismic trace, as seen on a 20x Develocorder film viewer. The curve plots the unit response, which is multiplied by a constant but known factor, CAL, to get the response for an individual station. Individual CAL factors for Type 1 seismographs are Develocorder equivalent peak-to-peak amplitudes, measured in millimeters, of a 100-microvolt 5 to $8-\mathrm{Hz}$ signal introduced to the preamp/VCO in place of the geophone at the field station. The calibration process is normally performed each time a station is visited for other required maintenance. 


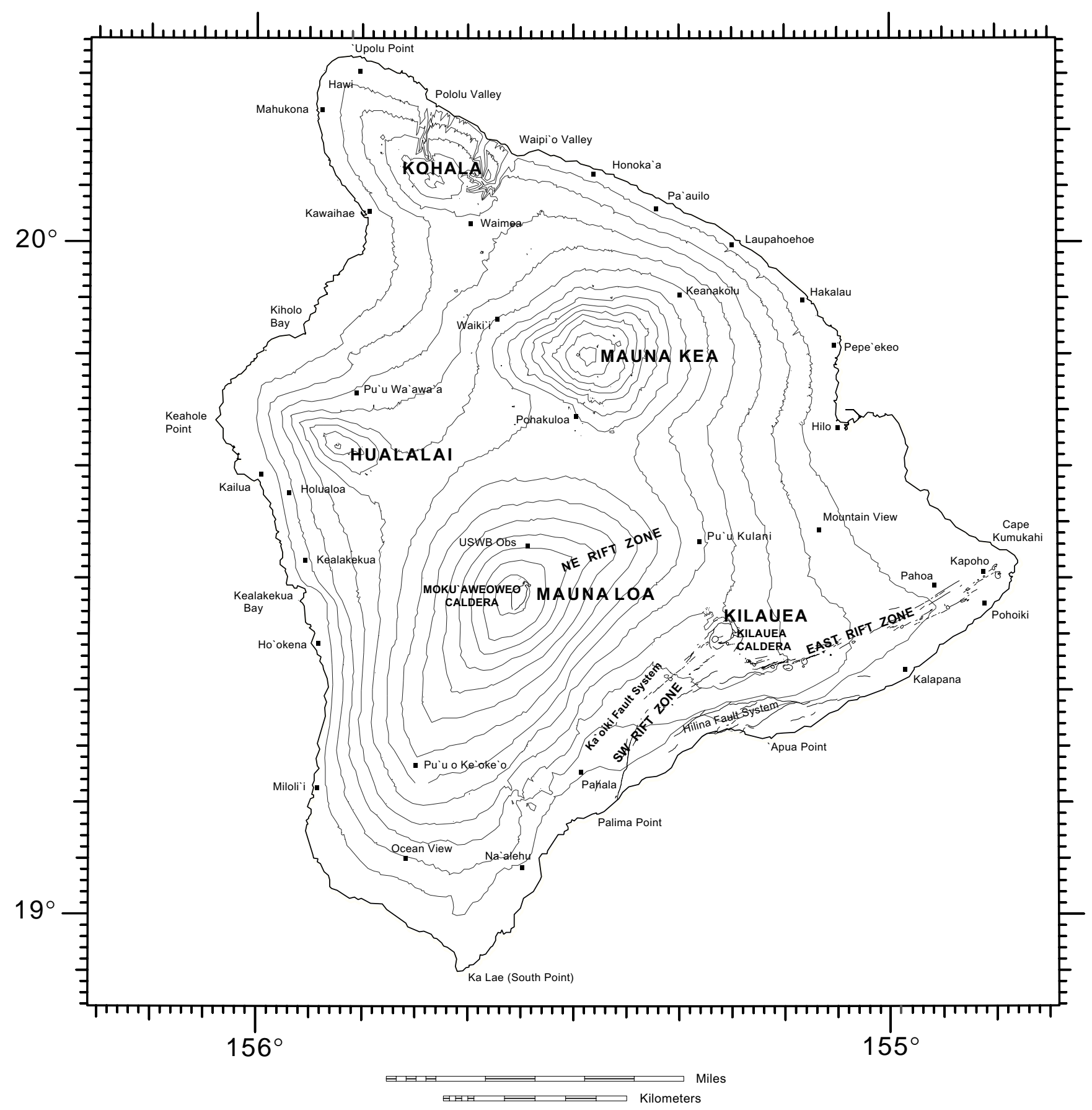

Figure 1. Map of the Island of Hawai $i$, showing principal settlements and selected geographic and geologic features. 


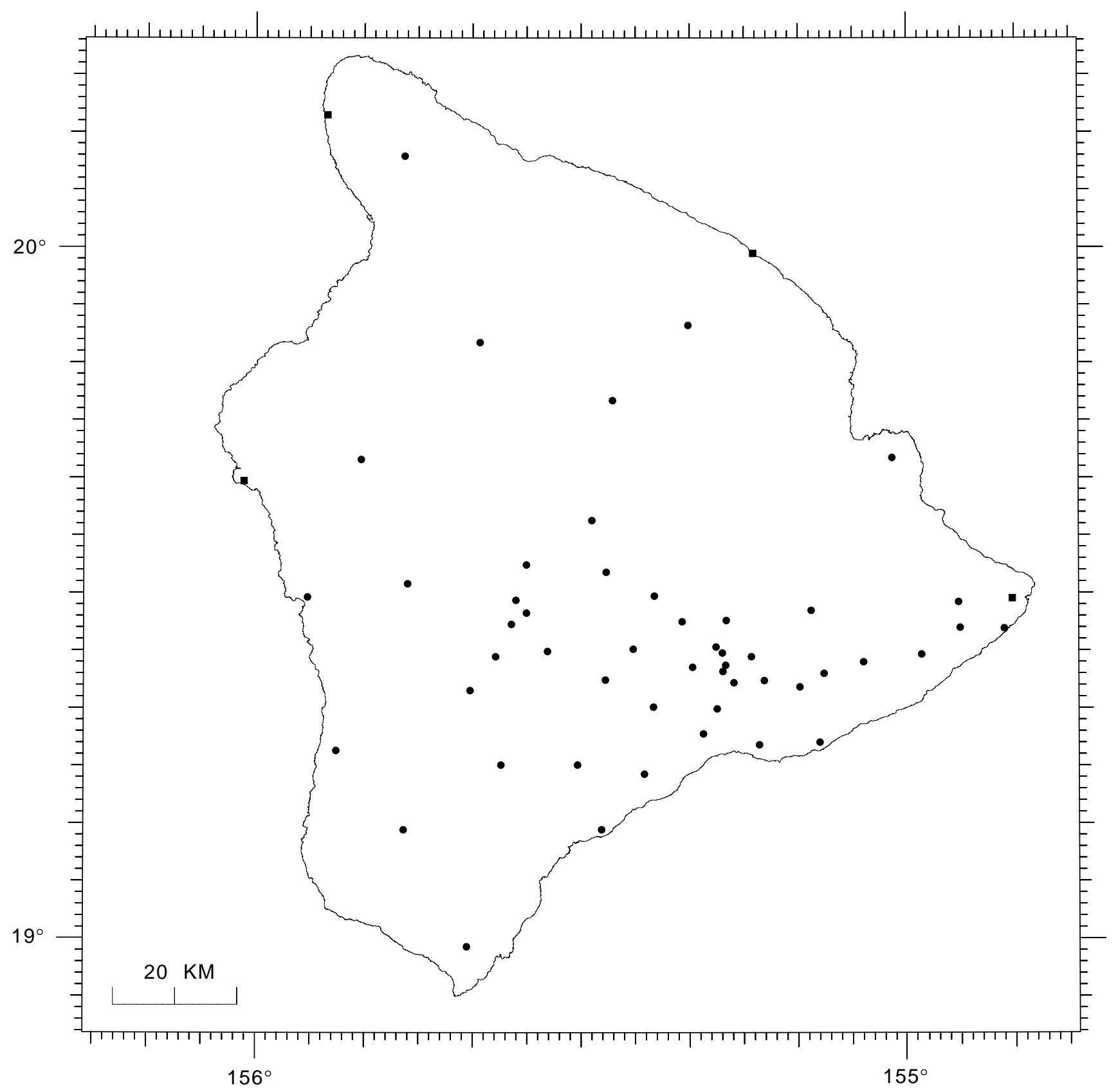

Figure 2. Seismic station sites operational during 2000 on the Island of Hawai'i. 


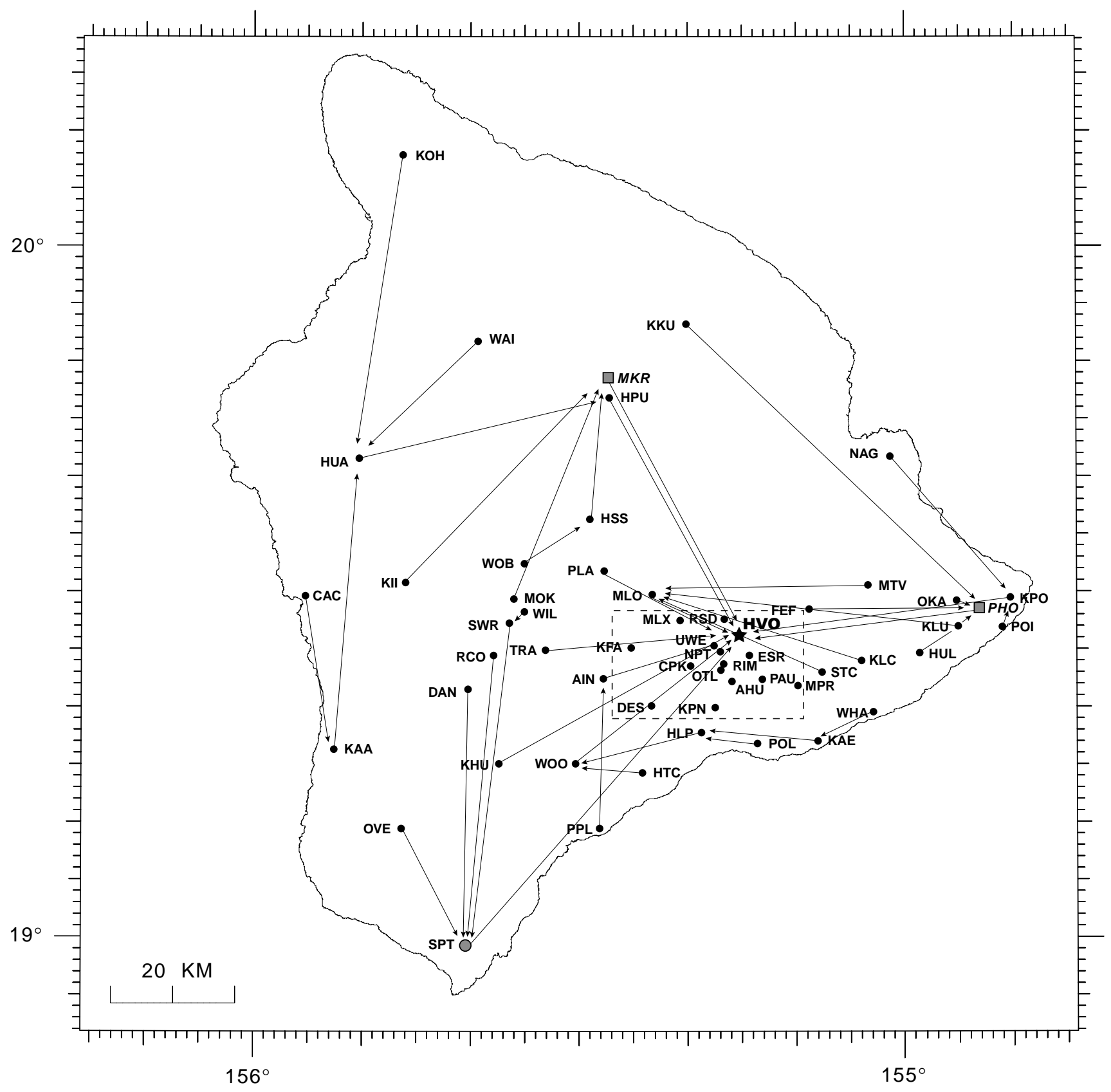

$\star \quad$ Hawaiian Volcano Observatory

- Network sites

O Direct -to-Line 32 Channel

$\square \quad$ Repeater sites

Inset Kilauea Summit

Figure 3. Telemetry scheme for seismic stations operational during 2000 on the Island of Hawai'i. 

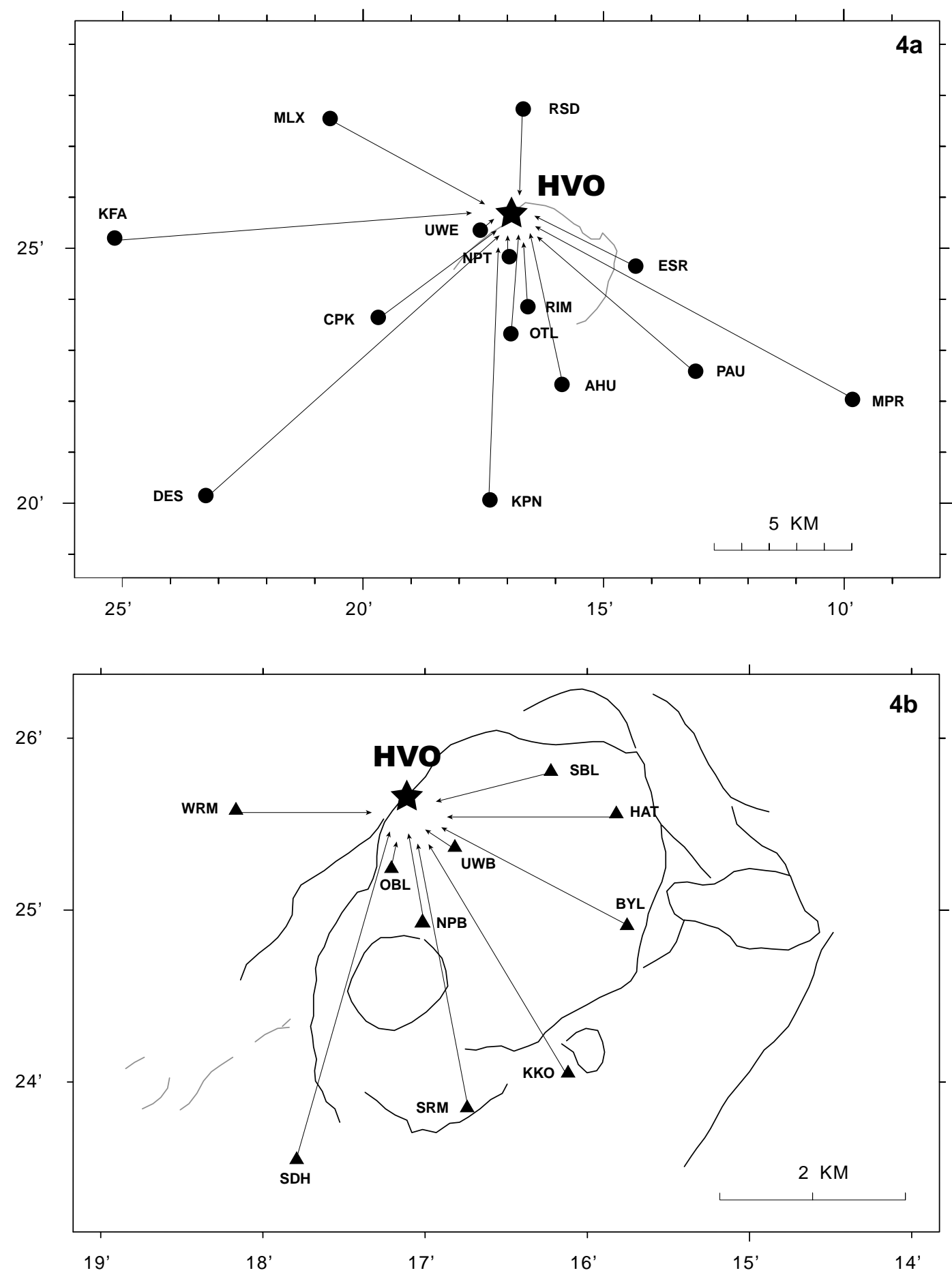

$\star \quad$ Hawaiian Volcano Observatory

Network sites

A Broadband sites

Figure 4a. Expanded telemetry scheme for the 2000 Hawaiian Volcano Observatory seismic network at Kilauea summit.

Figure 4b. Expanded telemetry scheme for the 2000 Menlo Park broadband network at Kilauea summit. 


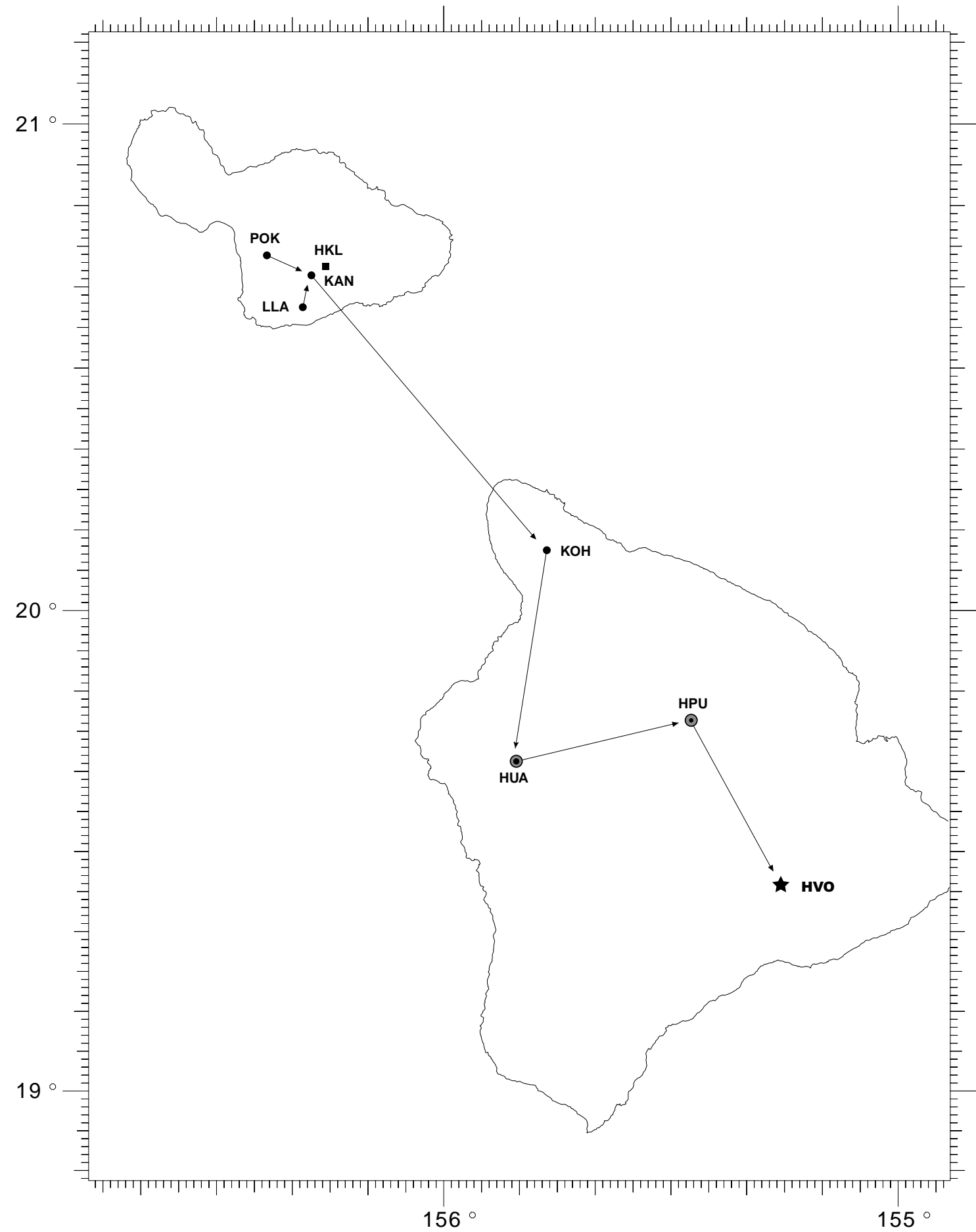

$\star \quad$ Hawaiian Volcano Observatory

- Network station sites

- Direct -to-Line 32 Channel

- PTWC station sites

Figure 5. Telemetry scheme for seismic stations operational during 2000 on the Island of Maui. 
Table 1. Seismic stations in Hawai'i operated by the USGS in 2000.

\begin{tabular}{|c|c|c|c|c|c|c|c|c|c|c|c|c|}
\hline \multirow[t]{2}{*}{ STATION NAME } & \multirow[t]{2}{*}{ CODE } & \multicolumn{2}{|c|}{ - LAT- } & -LON- & ELEV I & DELAY & DELAY & \multicolumn{2}{|c|}{ CAL SEIS } & \multicolumn{3}{|c|}{ OPTIC } \\
\hline & & $\mathrm{D}$ & $\mathrm{M}$ & $\mathrm{D}$ & M & $(\mathrm{M})$ & 1 & 2 & & TYPE & & RECORD \\
\hline AHUA & AHUV & 19 & 22.40 & 155 & 15.90 & 1070 & -0.10 & -0.13 & 2.6 & L5 & & I \\
\hline AHUA & AHUE & 19 & 22.40 & 155 & 15.90 & 1070 & -0.10 & -0.13 & 3.0 & E5 & MW & \\
\hline AHUA & AHUN & 19 & 22.40 & 155 & 15.90 & 1070 & -0.10 & -0.13 & 3.0 & E5 & MW & \\
\hline AINAPO & AINV & 19 & 22.50 & 155 & 27.62 & 1524 & 0.13 & 0.17 & 6.8 & L5 & & \\
\hline AINAPO & AINE & 19 & 22.50 & 155 & 27.62 & 1524 & 0.13 & 0.17 & 3.0 & L5 & $\mathrm{MW}$ & \\
\hline AINAPO & AINN & 19 & 22.50 & 155 & 27.62 & 1524 & 0.13 & 0.17 & 3.0 & L5 & MW & \\
\hline AINAPO & AINZ & 19 & 22.50 & 155 & 27.62 & 1524 & 0.13 & 0.17 & 0.0 & L5 & & \\
\hline CAPTAIN COOK & $\mathrm{CACV}$ & 19 & 29.29 & 155 & 55.09 & 323 & 0.00 & -0.16 & 1.1 & L5 & & \\
\hline CONE PEAK & $\mathrm{CPKV}$ & 19 & 23.70 & 155 & 19.70 & 1038 & -0.26 & -0.07 & 6.0 & L5 & & \\
\hline DANDELION & DANV & 19 & 21.42 & 155 & 40.04 & 3003 & -0.27 & 0.03 & 4.3 & E5 & & \\
\hline DESERT & DESV & 19 & 20.20 & 155 & 23.30 & 815 & -0.29 & -0.13 & 4.5 & L5 & & I \\
\hline DIAMOND HEAD, OA & DHHZ & 21 & 16.12 & 157 & 48.25 & 137 & 0.00 & 0.00 & 0.0 & S1 & & $\mathrm{H}$ \\
\hline ESCAPE ROAD & ESRV & 19 & 24.68 & 155 & 14.33 & 1177 & -0.17 & -0.19 & 1.2 & L5 & & \\
\hline FERN FOREST & FEFV & 19 & 28.70 & 155 & 8.91 & 691 & 0.01 & 0.05 & 0.0 & L5 & & \\
\hline HEIHEIAHULU & HHAZ & 19 & 25.13 & 154 & 58.72 & 369 & -0.17 & -0.16 & 0.0 & F5 & & \\
\hline HEIHEIAHULU & HHAE & 19 & 25.13 & 154 & 58.72 & 369 & -0.17 & -0.16 & 0.0 & F5 & & \\
\hline HEIHEIAHULU & HHAN & 19 & 25.13 & 154 & 58.72 & 369 & -0.17 & -0.16 & 0.0 & F5 & & \\
\hline HALEAKALA, MAUI & HKLZ & 20 & 42.63 & 156 & 15.55 & 3051 & 0.00 & 0.00 & 0.0 & S1 & & $\mathrm{H}$ \\
\hline HILINA PALI & HLPV & 19 & 17.96 & 155 & 18.63 & 707 & 0.02 & 0.07 & 2.1 & L5 & & \\
\hline HONOLULU, OAHU & HONZ & 21 & 19.30 & 158 & 0.50 & 2 & 0.00 & 0.00 & 0.0 & S1 & & $\mathrm{H}$ \\
\hline HONOLULU, OAHU & HONE & 21 & 19.30 & 158 & 0.50 & 2 & 0.00 & 0.00 & 0.0 & S1 & & $\mathrm{H}$ \\
\hline HONOLULU, OAHU & HONN & 21 & 19.30 & 158 & 0.50 & 2 & 0.00 & 0.00 & 0.0 & $\mathrm{~S} 1$ & & $\mathrm{H}$ \\
\hline HONUAPO & HPOZ & 19 & 5.34 & 155 & 33.23 & 15 & 0.00 & 0.00 & 0.0 & S1 & & \\
\hline HALE POHAKU & HPUV & 19 & 46.85 & 155 & 27.50 & 3396 & 0.31 & 0.17 & 3.3 & L5 & & \\
\hline HUMUULA SHEEP & HSSV & 19 & 36.31 & 155 & 29.13 & 2445 & 0.20 & 0.35 & 4.0 & L5 & & \\
\hline HUMUULA SHEEP & HSSE & 19 & 36.31 & 155 & 29.13 & 2445 & 0.20 & 0.35 & 3.0 & L5 & MW & \\
\hline HUMUULA SHEEP & HSSN & 19 & 36.31 & 155 & 29.13 & 2445 & 0.20 & 0.35 & 3.0 & L5 & MW & \\
\hline HOT CAVES & $\mathrm{HTCV}$ & 19 & 14.33 & 155 & 24.02 & 381 & -0.16 & -0.07 & 2.3 & E4 & & \\
\hline HUALALAI & HUAV & 19 & 41.25 & 155 & 50.32 & 2189 & 0.67 & 0.38 & 2.8 & L5 & & I \\
\hline HEIHEIAHULU & HULV & 19 & 25.13 & 154 & 58.72 & 369 & -0.17 & -0.16 & 1.6 & L5 & & $\mathrm{H}$ \\
\hline HEIHEIAHULU & HULE & 19 & 25.13 & 154 & 58.72 & 369 & -0.17 & -0.16 & 3.0 & E5 & $\mathrm{MW}$ & \\
\hline HEIHEIAHULU & HULN & 19 & 25.13 & 154 & 58.72 & 369 & -0.17 & -0.16 & 3.0 & L5 & MW & \\
\hline KAAPUNA & KAAV & 19 & 15.98 & 155 & 52.28 & 524 & -0.12 & -0.01 & 3.3 & E5 & & \\
\hline KAENA POINT & $\mathrm{KAEV}$ & 19 & 17.35 & 155 & 7.95 & 37 & -0.01 & 0.06 & 1.4 & L5 & & \\
\hline KANAHAU, MAUI & KANV & 20 & 41.60 & 156 & 17.48 & 2745 & 0.00 & 0.00 & 0.0 & L5 & & \\
\hline KAOIKI FAULTS & $\mathrm{KFAV}$ & 19 & 25.25 & 155 & 25.18 & 1579 & 0.13 & 0.17 & 0.0 & L5 & & \\
\hline KAHUKU & KHUV & 19 & 14.90 & 155 & 37.10 & 1939 & 0.03 & -0.03 & 5.0 & E5 & & \\
\hline KANEKII & KIIV & 19 & 30.56 & 155 & 45.90 & 1841 & 0.15 & 0.37 & 3.0 & L5 & & \\
\hline KANEKII & KIIE & 19 & 30.56 & 155 & 45.90 & 1841 & 0.15 & 0.37 & 3.0 & L5 & $\mathrm{MW}$ & \\
\hline KANEKII & KIIN & 19 & 30.56 & 155 & 45.90 & 1841 & 0.15 & 0.37 & 3.0 & L5 & $\mathrm{MW}$ & \\
\hline KIPAPA, OAHU & KIPZ & 21 & 25.40 & 158 & 0.90 & 2 & 0.00 & 0.00 & 0.0 & $\mathrm{ST}$ & & \\
\hline KAILUA, KONA & $\mathrm{KKHZ}$ & 19 & 39.40 & 156 & 1.12 & 1 & 0.00 & 0.00 & 0.0 & S1 & & \\
\hline KEANAKOLU & KKUV & 19 & 53.39 & 155 & 20.58 & 1863 & 0.68 & 0.24 & 3.3 & L5 & & \\
\hline KALALUA CONE & $\mathrm{KLCV}$ & 19 & 24.35 & 155 & 4.08 & 659 & -0.25 & -0.30 & 3.4 & L5 & & \\
\hline PUU KALIU & KLUV & 19 & 27.48 & 154 & 55.26 & 271 & -0.17 & -0.30 & 3.4 & L5 & & \\
\hline KOHALA & $\mathrm{KOHV}$ & 20 & 7.69 & 155 & 46.77 & 1166 & -0.03 & -0.17 & 6.3 & L5 & & \\
\hline KOHALA & $\mathrm{KOHE}$ & 20 & 7.69 & 155 & 46.77 & 1166 & -0.03 & -0.17 & 3.0 & L5 & $\mathrm{MW}$ & \\
\hline KOHALA & $\mathrm{KOHN}$ & 20 & 7.69 & 155 & 46.77 & 1166 & -0.03 & -0.17 & 3.0 & L5 & $\mathrm{MW}$ & \\
\hline KAPOHO CONE & $\mathrm{KPCZ}$ & 19 & 30.02 & 154 & 50.51 & 134 & 0.00 & 0.00 & 0.0 & S1 & & \\
\hline KIPUKA NENE & KPNV & 19 & 20.10 & 155 & 17.40 & 924 & -0.11 & -0.08 & 3.5 & L5 & & \\
\hline LUALAILUA, MAUI & LLAV & 20 & 37.62 & 156 & 18.62 & 683 & 0.00 & 0.00 & 0.0 & L5 & & \\
\hline LAUPAHOEHOE & LPHZ & 19 & 59.82 & 155 & 14.58 & 1 & 0.00 & 0.00 & 0.0 & S1 & & \\
\hline
\end{tabular}




\begin{tabular}{|c|c|c|c|c|c|c|c|c|c|c|c|c|}
\hline \multirow[t]{2}{*}{ STATION NAME } & \multirow[t]{2}{*}{ CODE } & \multicolumn{2}{|c|}{-LAT- } & \multirow{2}{*}{$\begin{array}{c}-\mathrm{LON}- \\
\mathrm{D}\end{array}$} & \multirow{2}{*}{$\begin{array}{c}\text { ELEV I } \\
\mathrm{M}\end{array}$} & \multirow{2}{*}{$\begin{array}{r}\text { DELAY I } \\
(\mathrm{M})\end{array}$} & \multirow{2}{*}{$\begin{array}{c}\text { DELAY } \\
1\end{array}$} & \multirow{2}{*}{\multicolumn{2}{|c|}{${ }_{2}^{\text {CAL SEIS }}$}} & \multirow{2}{*}{\multicolumn{2}{|c|}{$\begin{array}{l}\text { OPTIC } \\
\text { TYPE }\end{array}$}} & \\
\hline & & $\mathrm{D}$ & $\mathrm{M}$ & & & & & & & & & \\
\hline MAHUKONA & MHAZ & 20 & 11.27 & 155 & 54.18 & 1 & 0.00 & 0.00 & 0.0 & S1 & & \\
\hline MAUNA LOA & MLOV & 19 & 29.80 & 155 & 23.30 & 2010 & 0.03 & 0.08 & 5.6 & L5 & & I \\
\hline MAUNA LOA & MLOE & 19 & 29.80 & 155 & 23.30 & 2010 & 0.03 & 0.08 & 3.0 & L5 & MW & \\
\hline MAUNA LOA & MLON & 19 & 29.80 & 155 & 23.30 & 2010 & 0.03 & 0.08 & 3.0 & L5 & MW & \\
\hline MAUNA LOA $X$ & MLXV & 19 & 27.60 & 155 & 20.70 & 1475 & 0.06 & 0.15 & 3.0 & L5 & & \\
\hline MOKUAWEOWEO & MOKV & 19 & 29.28 & 155 & 35.98 & 4104 & 0.15 & 0.16 & 4.2 & L5 & & IH \\
\hline MAKAOPUHI & MPRV & 19 & 22.07 & 155 & 9.85 & 881 & -0.17 & -0.20 & 2.6 & L5 & & I \\
\hline MAKAOPUHI & MPRZ & 19 & 22.07 & 155 & 9.85 & 881 & -0.17 & -0.20 & 0.1 & L5 & & \\
\hline NATIONAL GUARD & NAGV & 19 & 42.12 & 155 & 1.72 & 18 & 0.54 & 0.30 & 4.0 & R5 & & \\
\hline NATIONAL GUARD & NAGE & 19 & 42.12 & 155 & 1.72 & 18 & 0.54 & 0.30 & 3.0 & R5 & MW & \\
\hline NATIONAL GUARD & NAGN & 19 & 42.12 & 155 & 1.72 & 18 & 0.54 & 0.30 & 3.0 & R5 & MW & \\
\hline NORTH PIT & NPTV & 19 & 24.90 & 155 & 17.00 & 1115 & -0.30 & -0.18 & 3.0 & L5 & & IH \\
\hline NORTH PIT & NPTE & 19 & 24.90 & 155 & 17.00 & 1115 & -0.30 & -0.18 & 3.0 & L5 & MW & \\
\hline NORTH PIT & NPTN & 19 & 24.90 & 155 & 17.00 & 1115 & -0.30 & -0.18 & 3.0 & L5 & MW & \\
\hline OOKA & OKAV & 19 & 29.66 & 154 & 55.44 & 180 & 0.00 & 0.00 & 0.0 & L5 & & \\
\hline OPANA, OAHU & OPAZ & 21 & 41.45 & 158 & 0.70 & 100 & 0.00 & 0.00 & 0.0 & S1 & & $\mathrm{H}$ \\
\hline OUTLET & OTLV & 19 & 23.38 & 155 & 16.94 & 1038 & -0.19 & -0.18 & 2.6 & L5 & & \\
\hline OUTLET & OTLZ & 19 & 23.38 & 155 & 16.94 & 1038 & -0.19 & -0.18 & 0.0 & L5 & & \\
\hline OCEANVIEW EST & OVEV & 19 & 9.21 & 155 & 45.92 & 1378 & 0.00 & 0.00 & 0.0 & L5 & & \\
\hline PAUAHI & PAAZ & 19 & 22.62 & 155 & 13.10 & 994 & -0.21 & -0.24 & 0.0 & F5 & & \\
\hline PAUAHI & PAAE & 19 & 22.62 & 155 & 13.10 & 994 & -0.21 & -0.24 & 0.0 & F5 & & \\
\hline PAUAH I & PAAN & 19 & 22.62 & 155 & 13.10 & 994 & -0.21 & -0.24 & 0.0 & F5 & & \\
\hline PAUAHI & PAUV & 19 & 22.62 & 155 & 13.10 & 994 & -0.21 & -0.24 & 2.9 & L4 & & \\
\hline PAUAHI & PAUE & 19 & 22.62 & 155 & 13.10 & 994 & -0.21 & -0.24 & 3.0 & L5 & MW & \\
\hline PAUAHI & PAUN & 19 & 22.62 & 155 & 13.10 & 994 & -0.21 & -0.24 & 3.0 & L5 & MW & \\
\hline PUU ULAULA & PLAV & 19 & 32.00 & 155 & 27.67 & 2992 & -0.03 & 0.13 & 6.3 & L5 & & I \\
\hline POHOIKI & POIV & 19 & 27.42 & 154 & 51.22 & 16 & -0.09 & -0.24 & 0.0 & L5 & & \\
\hline PUUOKALI, MAUI & POKV & 20 & 44.00 & 156 & 23.32 & 511 & 0.00 & 0.00 & 0.0 & L5 & & \\
\hline POLIOKEAWE PALI & POLV & 19 & 17.02 & 155 & 13.47 & 169 & -0.02 & 0.03 & 3.4 & E5 & & \\
\hline PUU PILI & PPLV & 19 & 9.50 & 155 & 27.87 & 35 & -0.15 & -0.15 & 1.4 & E5 & & \\
\hline RED CONE & $\mathrm{RCOV}$ & 19 & 24.36 & 155 & 37.79 & 3601 & 0.00 & 0.00 & 0.0 & L5 & & \\
\hline RIM & RIMV & 19 & 23.90 & 155 & 16.60 & 1128 & -0.21 & -0.13 & 0.0 & L5 & & \\
\hline RAINSHED & RSDV & 19 & 27.78 & 155 & 16.68 & 1270 & 0.06 & 0.15 & 0.0 & L5 & & \\
\hline SOUTH POINT & SPTV & 18 & 58.91 & 155 & 39.92 & 244 & -0.17 & -0.22 & 2.8 & L5 & & \\
\hline SOUTH POINT & SPTE & 18 & 58.91 & 155 & 39.92 & 244 & -0.17 & -0.22 & 3.0 & L5 & MW & \\
\hline SOUTH POINT & SPTN & 18 & 58.91 & 155 & 39.92 & 244 & -0.17 & -0.22 & 3.0 & L5 & MW & \\
\hline STEAM CRACKS & $\mathrm{STCV}$ & 19 & 23.30 & 155 & 7.67 & 765 & -0.25 & -0.30 & 3.4 & L5 & & $\mathrm{H}$ \\
\hline STEAM CRACKS & STCE & 19 & 23.30 & 155 & 7.67 & 765 & -0.25 & $5-0.30$ & 3.0 & L5 & MW & \\
\hline STEAM CRACKS & $\mathrm{STCN}$ & 19 & 23.30 & 155 & 7.67 & 765 & -0.25 & $5-0.30$ & 3.0 & L5 & MW & \\
\hline SOUTHWEST RIFT & SWRV & 19 & 27.26 & 155 & 36.30 & 4048 & 0.01 & 0.04 & 5.6 & E5 & & \\
\hline TRAIL & TRAV & 19 & 24.91 & 155 & 32.96 & 3207 & 0.00 & 0.00 & 0.0 & L5 & & \\
\hline UWEKAHUNA & URAV & 19 & 25.40 & 155 & 17.60 & 1240 & -0.21 & 0.00 & 0.0 & R5 & & \\
\hline UWEKAHUNA & URAE & 19 & 25.40 & 155 & 17.60 & 1240 & -0.21 & 0.00 & 3.0 & R5 & MW & \\
\hline UWEKAHUNA & URAN & 19 & 25.40 & 155 & 17.60 & 1240 & -0.21 & 0.00 & 3.0 & R5 & MW & \\
\hline UWEKAHUNA & UUGZ & 19 & 25.40 & 155 & 17.60 & 1240 & 0.00 & 0.00 & 0.0 & L0 & & \\
\hline WAIKII & WAIV & 19 & 51.58 & 155 & 39.60 & 1433 & 0.20 & 0.35 & 0.0 & L5 & & \\
\hline WILKES CAMP & WILV & 19 & 28.15 & 155 & 35.02 & 4037 & 0.22 & 0.17 & 2.6 & E5 & & \\
\hline WILKES CAMP & WILE & 19 & 28.15 & 155 & 35.02 & 4037 & 0.22 & 0.17 & 3.0 & L5 & MW & \\
\hline WILKES CAMP & WILN & 19 & 28.15 & 155 & 35.02 & 4037 & 0.22 & 0.17 & 3.0 & L5 & MW & \\
\hline WAIMANALO RG, OA & WMRZ & 21 & 19.22 & 157 & 40.94 & 200 & 0.00 & 0.00 & 0.0 & S1 & & \\
\hline WEATHER OBSERV & WOBV & 19 & 32.31 & 155 & 35.01 & 3396 & 0.00 & 0.00 & 0.0 & E5 & & \\
\hline WOOD VALLEY & WOOV & 19 & 15.08 & 155 & 30.12 & 909 & -0.15 & -0.06 & 2.6 & E5 & & \\
\hline
\end{tabular}




\section{Table 2. Seismic instrument types}

The codes in parentheses refer to the seismometer types listed in Table 1.

Type 1 (Codes E, L, R, and 4, 5) consists of:

a) Geophone - Electrotech EV-17 (E), Mark Products L4C (L) or Kinemetric Ranger SS1 (R).

$(\mathrm{L})$ and $(\mathrm{R})$ are 1.0-sec. period moving-magnet vertical- or horizontal- (E-W and N-

S) component seismometers adjusted for an output of 0.5 volts $/ \mathrm{cm} / \mathrm{sec}$ and 0.8 , critically damped.

b) Preamp/VCO - USGS/OEVE Model J402 (4), J502 (5) voltage-controlled oscillator.

Three $\mathrm{db}$ points for bandpass filter at $0.1 \mathrm{~Hz}$ and $30 \mathrm{~Hz}$. Signals are transmitted on audio FM carrier over cable or FM radio link to HVO.

Code (W) - Wood-Anderson torsion seismograph.

Code (MW) - Horizontal-component seismograph based on a Type 1 system and modified to $3 \mathrm{x}$ a Wood-Anderson response.

Code (F) - Kinemetric Force-Balance Accelerometer (FBA23).

Code (S13) - Geotech, $1 \mathrm{~Hz}$ seismometer with A1 VCO operated by the Pacific Tsunami Warning Center.

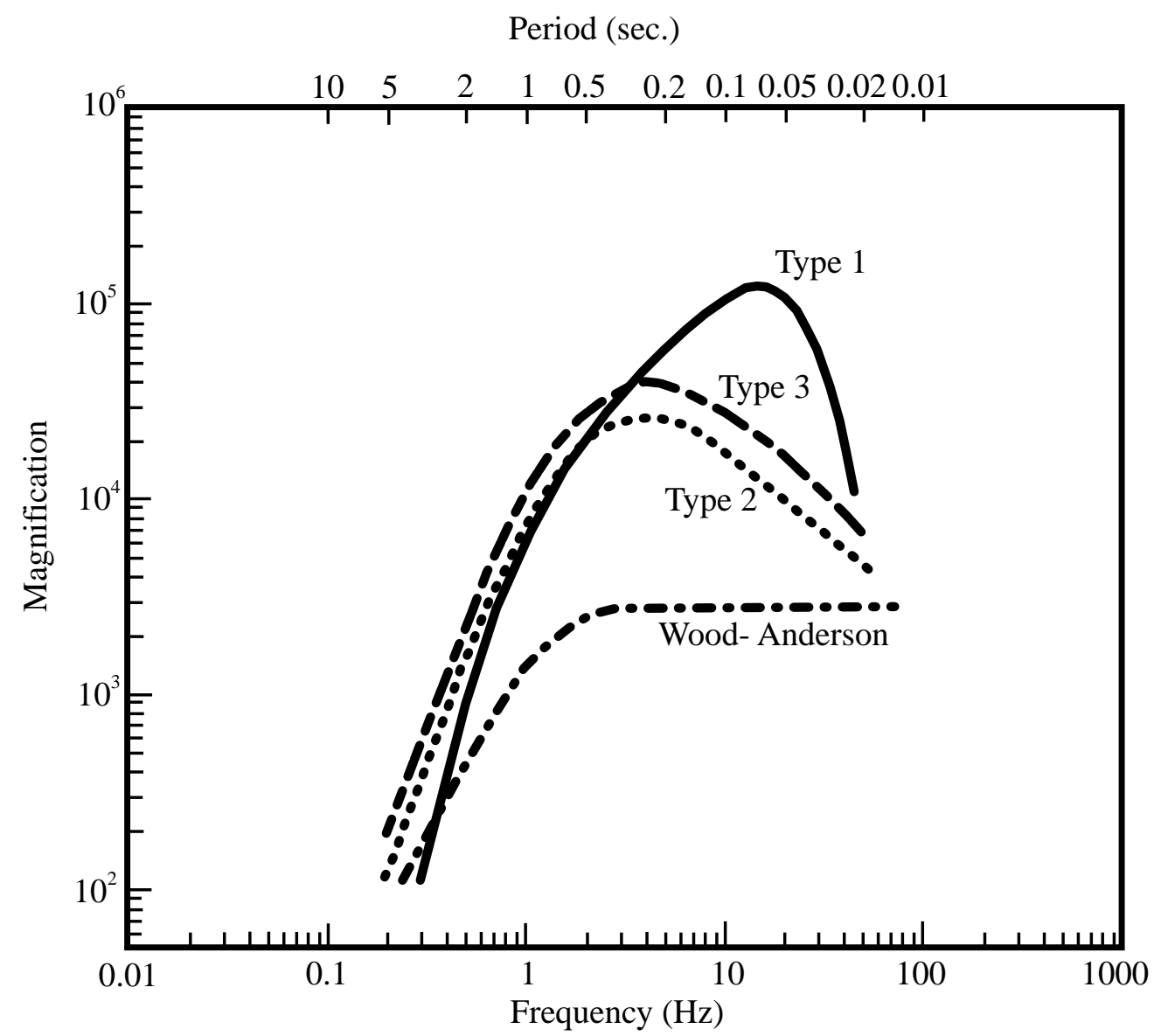

Figure 6. System-response curves for the Wood-Anderson torsion seismograph and for seismometers used by the Hawaiian Volcano Observatory. Type 1 is the standard OEVE seismometer system recorded on Develocorder film and DAT tape. The curve for Type 1 includes response of the geophone, all electronics including telemetry, Develocorder galvanometer, and projection of film by a $20 \mathrm{x}$ viewer. The curve plots the unit response, which should be multiplied by a constant but known factor (CAL) to get the response for an individual station. 


\section{SEISMIC DATA PROCESSING}

Due to age and high cost of maintanance, Develocorder 'A' was discontinued on August 1, 1997. Daily count of classified micoearthquakes from source regions around Kilauea and Mauna Loa, and duration of tremor, were also discontinued. Coda duration, however, is measured in seconds from drum (ink or helicorder) records to determine a coda magnitude that is entered as an external magnitude in the final solution.

In 1986, HVO acquired a VAX 11-750 computer and adopted the CUSP (California Institute of Technology USGS Seismic Processing) routine. Discriminated analog signals are converted to digital form, and detected events are saved in real time. Detected events are demultiplexed, and P-picks are made by the computer, producing a rough location. Events are examined by an analyst, on a graphics terminal, to refine computer Ppicks and to time additional P- and S-phases for a preliminary location. Binary CUSP files are tape-archived and translated into ASCII phase files. Locations and amplitude magnitudes are then determined, using the program HYPOINVERSE (Klein, 2000) ${ }^{2}$. Events are reworked and rerun, as needed, to produce a final solution. Magneto-optical copies of arrival times and output summary data are kept at HVO.

In July 1992, HVO acquired VAX workstations for timing earthquakes using a "generic" version of CUSP. In addition to timing $\mathrm{P}$ and $\mathrm{S}$ arrival signals, the $\mathrm{VAX}$ workstations are capable of measuring peak-to-peak amplitudes along with the associated period. This capability allowed the renewal of amplitude magnitude determinations from the network seismic stations. Amplitude data gathered from July 1992 to July 1997 became part of a test set to determine magnitude corrections for network stations. Results of newly determined magnitude corrections are detailed by Nakata and Okubo (1997) ${ }^{3}$.

The crustal model used is specified by velocities at four depth points. Velocity at any depth is given by linear interpolation between points and uses a homogeneous half-space, as listed below:

$\begin{array}{cc}\begin{array}{c}\text { VELOCITY } \\ (\mathrm{km} / \mathrm{sec})\end{array} & \begin{array}{c}\text { DEPTH } \\ (\mathrm{km})\end{array} \\ 6.9 & 0.0 \\ 6.9 & 4.6 \\ 8.3 & 15.0 \\ & \geq 16.5\end{array}$

Two empirical sets of station delays or corrections were used in the HYPOINVERSE locations and are given in table 1 . The delay models are separated by a circle of radius $34 \mathrm{~km}$, centered at $19^{\circ} 22^{\prime} \mathrm{N}$ and $155^{\circ} 10^{\prime} \mathrm{W}$. Delay model 1 is used for epicenters occurring within a circle of radius $31 \mathrm{~km}$ from the center. This region includes Kilauea and its south flank. A combination of the two delay models is used for epicenters that fall in a transition zone that is $6 \mathrm{~km}$ wide. Delay model 2 is applied to the rest of the island and offshore earthquakes. For a detailed description, refer to Klein (in press) ${ }^{2}$.

Magnitudes for events are computed using recorded amplitudes on selected network vertical, Modified Wood-Anderson (MW) horizontal, and/or moderate and low gain stations. Amplitude readings are corrected to an equivalent Wood-Anderson amplitude using the curves of figure 6 and CAL factors listed in table 1.

Duration magnitude is determined by the length of signal, in seconds, read from drum recordings of Type 1 seismographs. This length of time is measured from the $\mathrm{P}$ arrival to the point where the earthquake signal has decayed to nearly the background noise level. Drum-recorded duration magnitude is calculated with a relationship equivalent to the develocorder viewer output.

\footnotetext{
2 Klein, F.W., in press, User's guide to HYPOINVERSE-2000, a Fortran Program to solve for earthquake locations and magnitudes: U.S. Geological Survey, $116 \mathrm{p}$.

3 Nakata, J., and Okubo, P., 1997, Determination of station amplitude magnitude corrections for the Hawaiian Volcano Observatory telemetered seismograph network: Data from 1992-1997: U.S. Geological Survey OpenFile Report 97-863, 73 p.
} 


\section{SEISMIC CATALOG}

The emphasis in both station coverage and detailed data analysis is on the highly active south half of the Island of Hawai' $i$. The set of well-recorded earthquakes located in the Hawai' $i$ Island region is nearly complete above magnitude 2.0. Many smaller events are located in the densely instrumented Kilauea area. Substantial effort is made to locate earthquakes elsewhere within the Hawaiian Archipelago. Such coverage cannot be as complete as in south Hawai'i, but nearly all events above magnitude 4.0 are located with limited precision.

Data presented in the seismic catalog are in three parts: (1) Maps showing computer-located hypocenters are given in figures 11-24. The location maps are of different scales and provide hypocenters with magnitude thresholds set at 1.0, 2.0, 3.0, and 3.5, varying according to region. (2) The list of computer locations constitutes the bulk of this summary and is given in table 4. Each earthquake in the list is assigned a threeletter code based on its general location and depth. Figures 7-10 are maps of the regions used to assign the location codes. The latitude and longitude limits of rectangular regions are listed in table 3 . When the listed coordinates overlap, precedence is given according to figures 7-10. (3) Table 5 re-lists the events in table 4 for which the preferred magnitude is 3.0 or larger. This list includes many of the earthquakes felt in Hawai' $i$.

Table 3. Names and coordinates of regions used for classifying earthquakes.

All earthquakes locate in one of the following groups, identified by a numerical class or three-letter code:

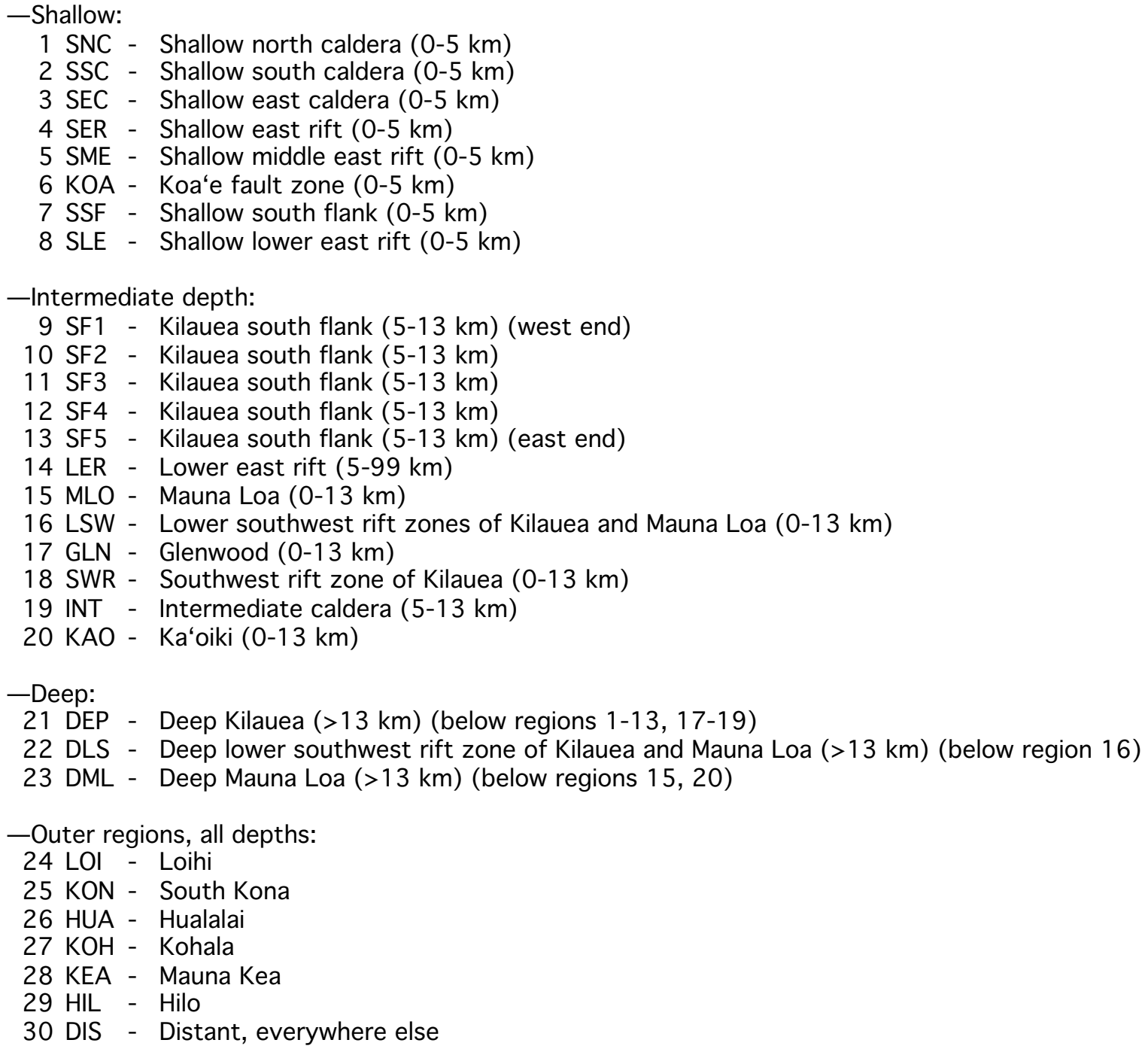


Table 3 (continued). The latitude and longitude limits of the regions are given below. If the coordinates overlap, precedence is given according to maps in figures 7-10.

$\begin{array}{rlllll}\text { No. } & \text { Code } & \text { N. Lat. } & \text { S. Lat. } & \text { W. Lon. } & \text { E. Lon. } \\ 1 & \text { SNC } & 1928.0 & 1924.5 & 15519.0 & 15514.0 \\ 2 & \text { SSC } & 1924.5 & 1922.0 & 15519.0 & 15516.5 \\ 3 & \text { SEC } & 1924.5 & 1922.0 & 15516.5 & 15514.0 \\ 4 & \text { SER } & 1926.0 & 1920.5 & 15514.0 & 15507.2 \\ 5 & \text { SME } & 1926.0 & -19 & 15507.2 & 15500.0 \\ 6 & \text { KOA } & 1922.0 & 1920.5 & 15517.0 & 15514.0 \\ 7 & \text { SSF } & 19 & 1910.0 & 15517.0 & 15500.0 \\ 8 & \text { SLE } & 1932.0 & 1916.0 & 15500.0 & 15440.0 \\ 9 & \text { SF1 } & 1922.0 & 1910.0 & 15517.0 & 15514.5 \\ 10 & \text { SF2 } & 1926.0 & 1910.0 & 15514.5 & 15512.3 \\ 11 & \text { SF3 } & 1926.0 & 1910.0 & 15512.3 & 15509.1 \\ 12 & \text { SF4 } & 1926.0 & 1910.0 & 15509.1 & 15505.3 \\ 13 & \text { SF5 } & 1926.0 & 1910.0 & 15505.3 & 15500.0 \\ 14 & \text { LER } & 1932.0 & 1916.0 & 15500.0 & 15440.0 \\ 15 & \text { MLO } & 1935.0 & 1919.0 & 15535.0 & 15519.0 \\ 16 & \text { LSW } & 1919.0 & 1840.0 & 15543.0 & 15525.0 \\ 17 & \text { GLN } & 1935.0 & 1926.0 & 15519.0 & 15500.0 \\ 18 & \text { SWR } & 1922.0 & 1910.0 & 15525.0 & 15517.0 \\ 19 & \text { INT } & 1928.0 & 1922.0 & 15519.0 & 15514.0 \\ 20 & \text { KAO } & 1930.0 & 1919.0 & 15532.0 & 15519.0 \\ 21 & \text { DEP } & 1935.0 & 1910.0 & 15525.0 & 15500.0 \\ 22 & \text { DLS } & 1919.0 & 1840.0 & 15543.0 & 15525.0 \\ 23 & \text { DML } & 1935.0 & 1919.0 & 15535.0 & 15519.0 \\ 24 & \text { LOI } & 1910.0 & 1840.0 & 15525.0 & 15500.0 \\ 25 & \text { KON } & 1939.0 & 1900.0 & 15620.0 & 15543.0 \\ 26 & \text { HUA } & 1955.0 & 1939.0 & 15620.0 & 15543.0 \\ 27 & \text { KOH } & 2025.0 & 1955.0 & 15620.0 & 15534.0 \\ 28 & \text { KEA } & 2025.0 & 1935.0 & 15534.0 & 15440.0 \\ 29 & \text { HIL } & 1947.0 & 1932.0 & 15509.0 & 15440.0 \\ & & & & & \end{array}$




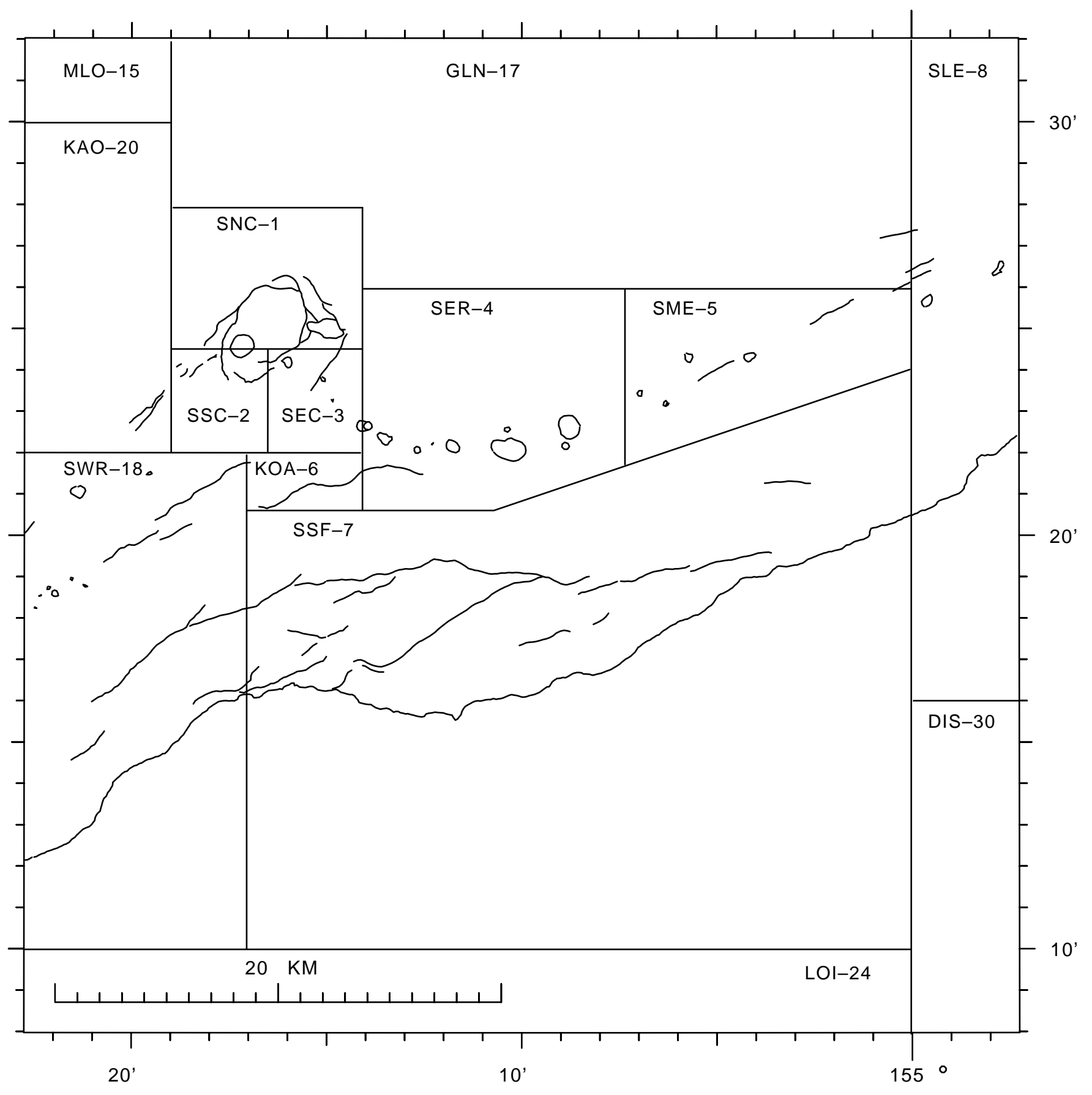

Figure 7. Earthquake classification, shallow (0-5 km deep), for Kilauea and the east flank of Mauna Loa. 


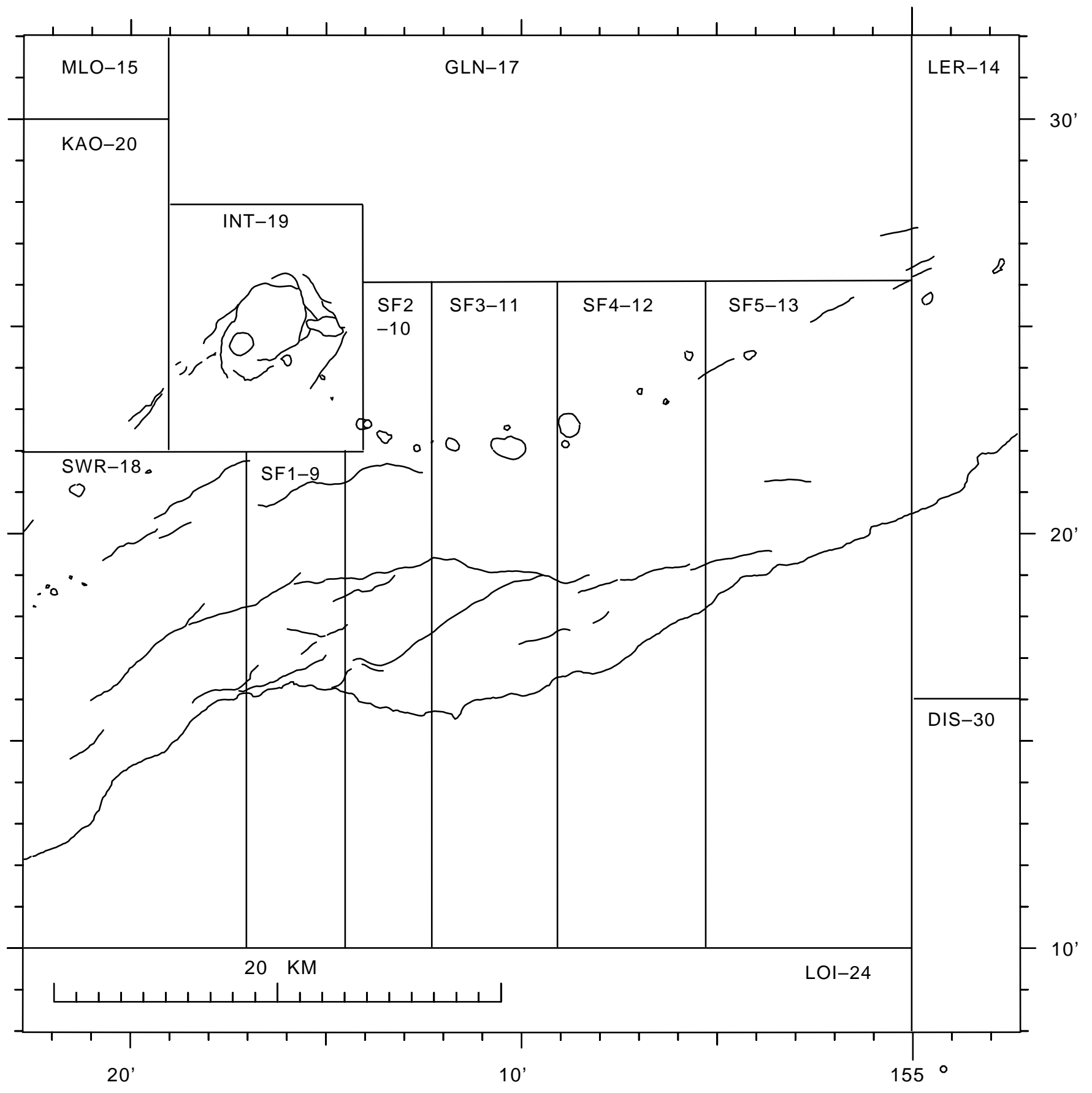

Figure 8. Earthquake classification, intermediate (5.1-13 km deep), for Kilauea and the east flank of Mauna Loa. 


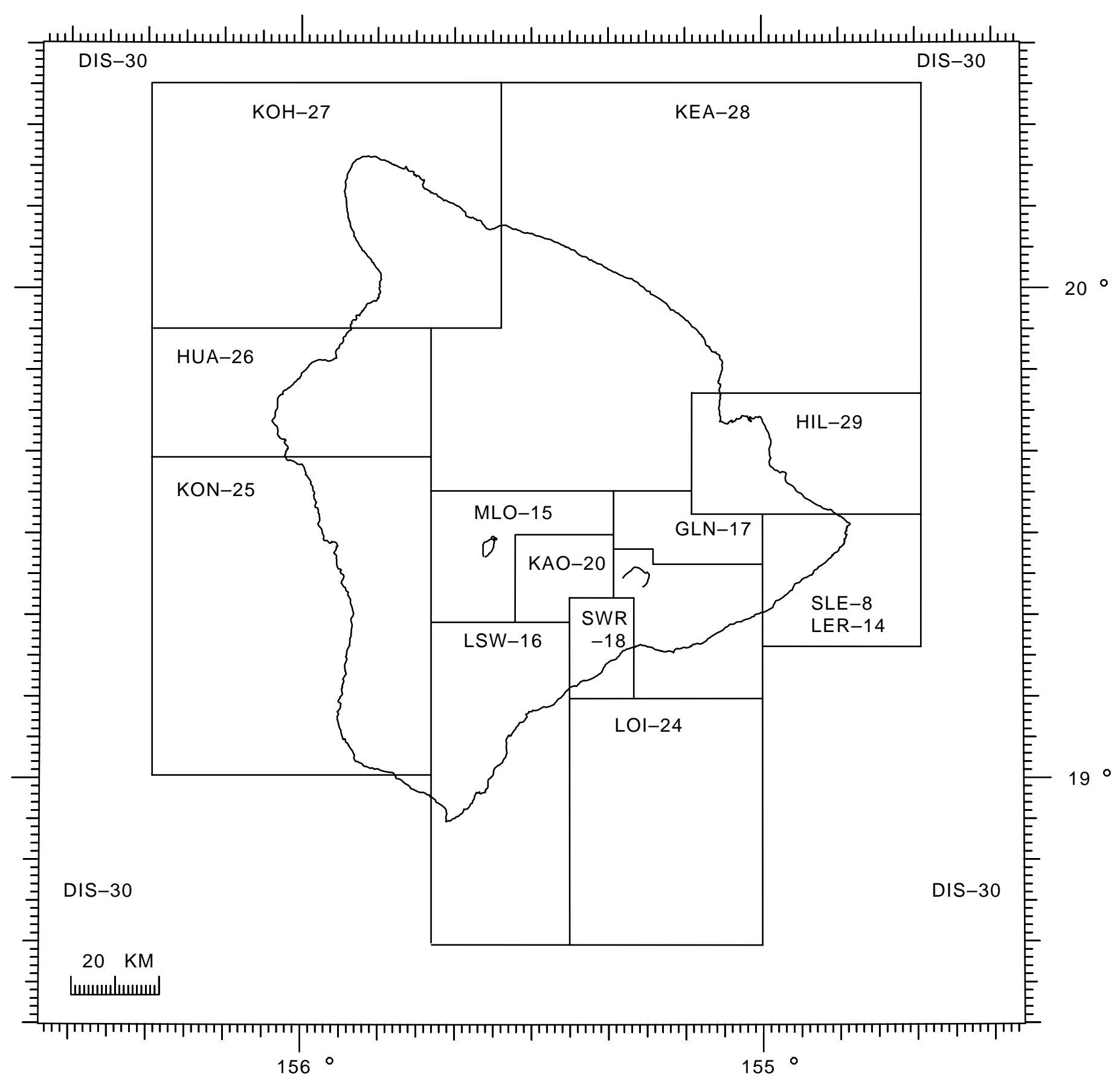

Figure 9. Earthquake classification, crustal (0-13 km deep), for the Island of Hawai' $i$. 


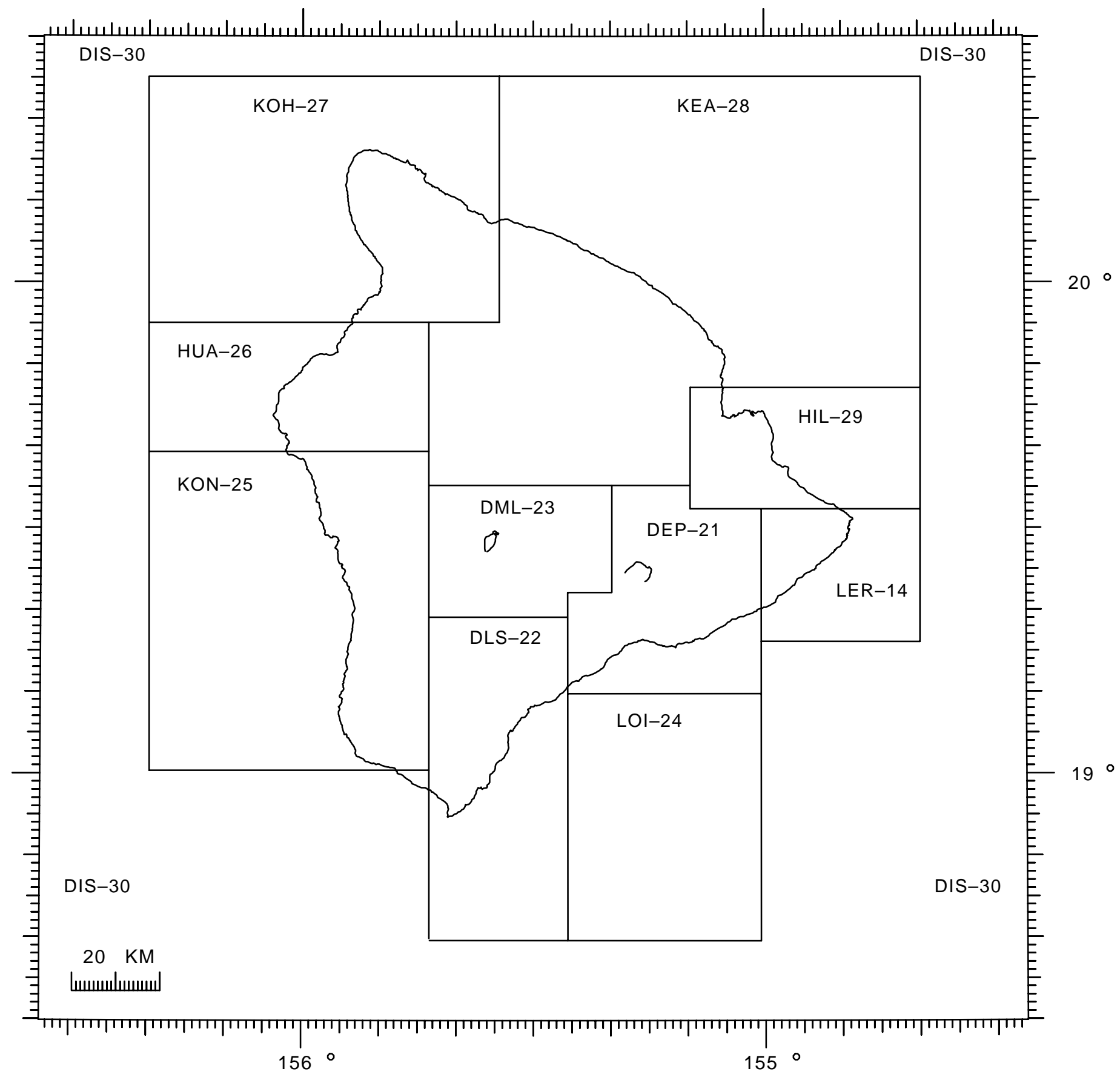

Figure 10. Earthquake classification, deep (greater than $13 \mathrm{~km}$ deep), for the Island of Hawai $i$. 
Figure 11. 2000 earthquake locations, Hawaiian Islands,

0-60 km depth, $M>=3.5$.

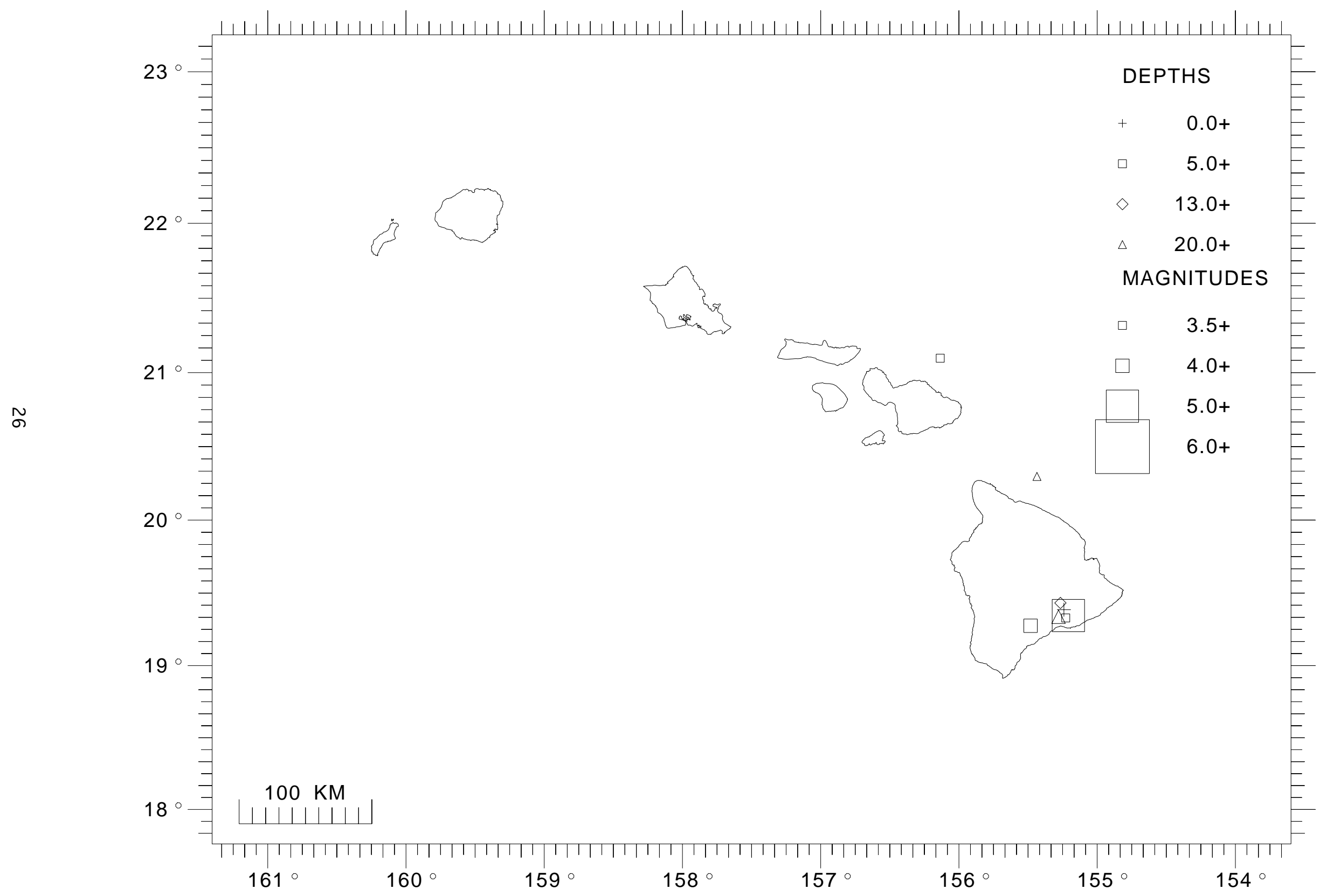


Figure 12. 2000 earthquake locations, Hawai'i Island,

$0-60 \mathrm{~km}$ depth, $M>=3.0$.

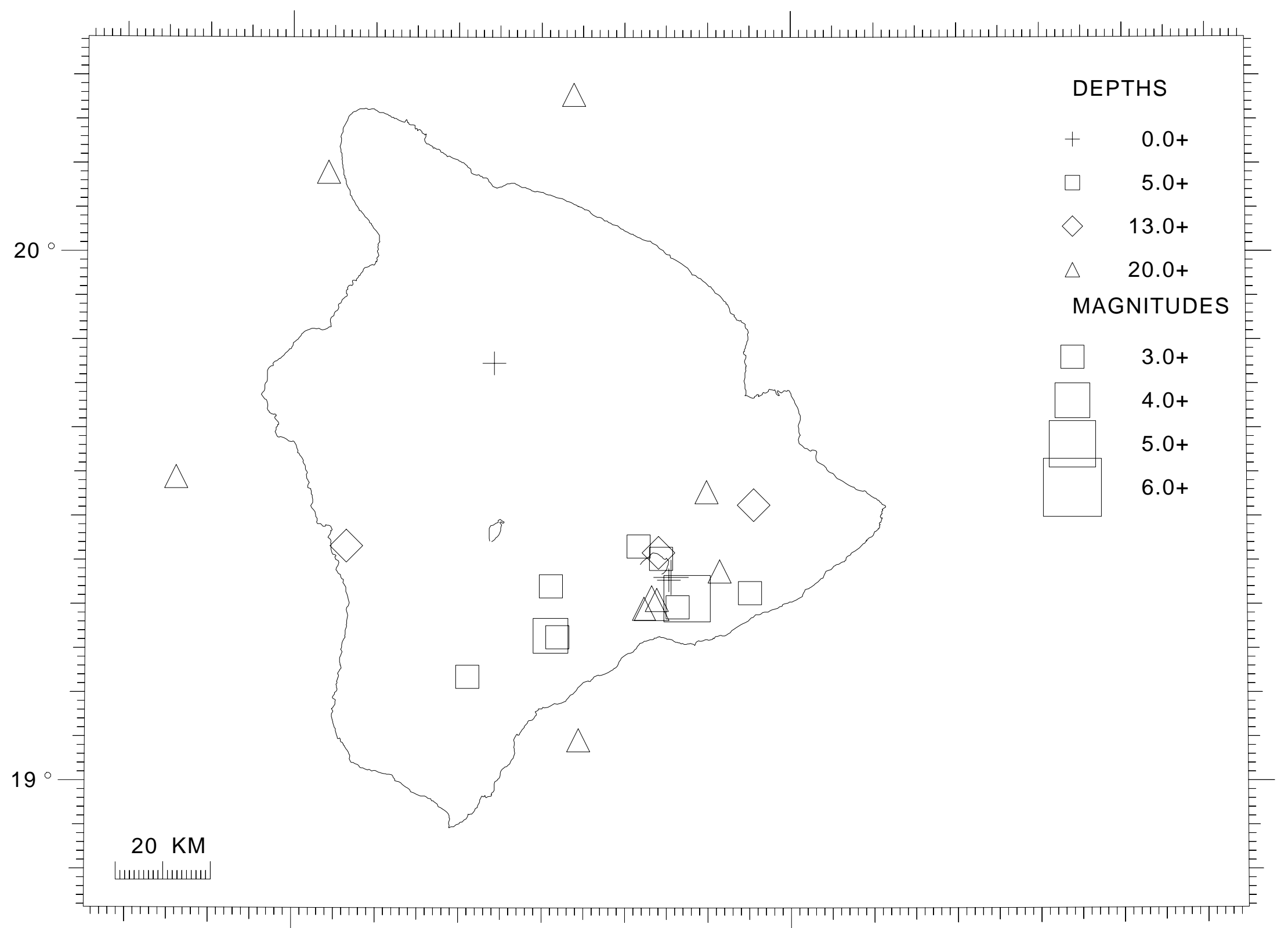


Figure 13. 2000 earthquake locations, Hawai'i Island, shallow $(0-5.0 \mathrm{~km}$ depth), $M>=2.0$.

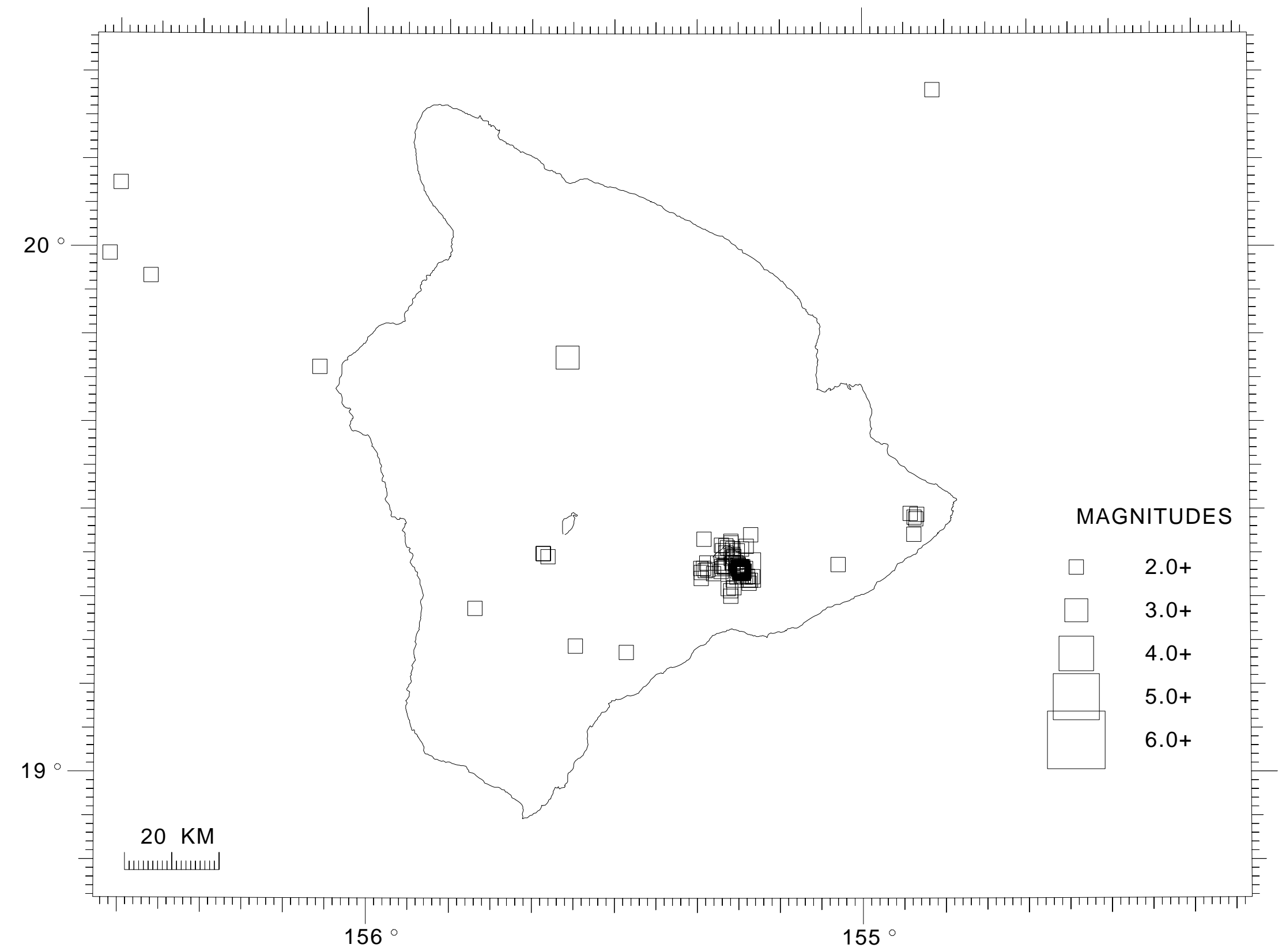


Figure 14. 2000 earthquake locations, Hawai'i Island, intermediate $(5.1-13.0 \mathrm{~km}$ depth), $M>=2.0$.

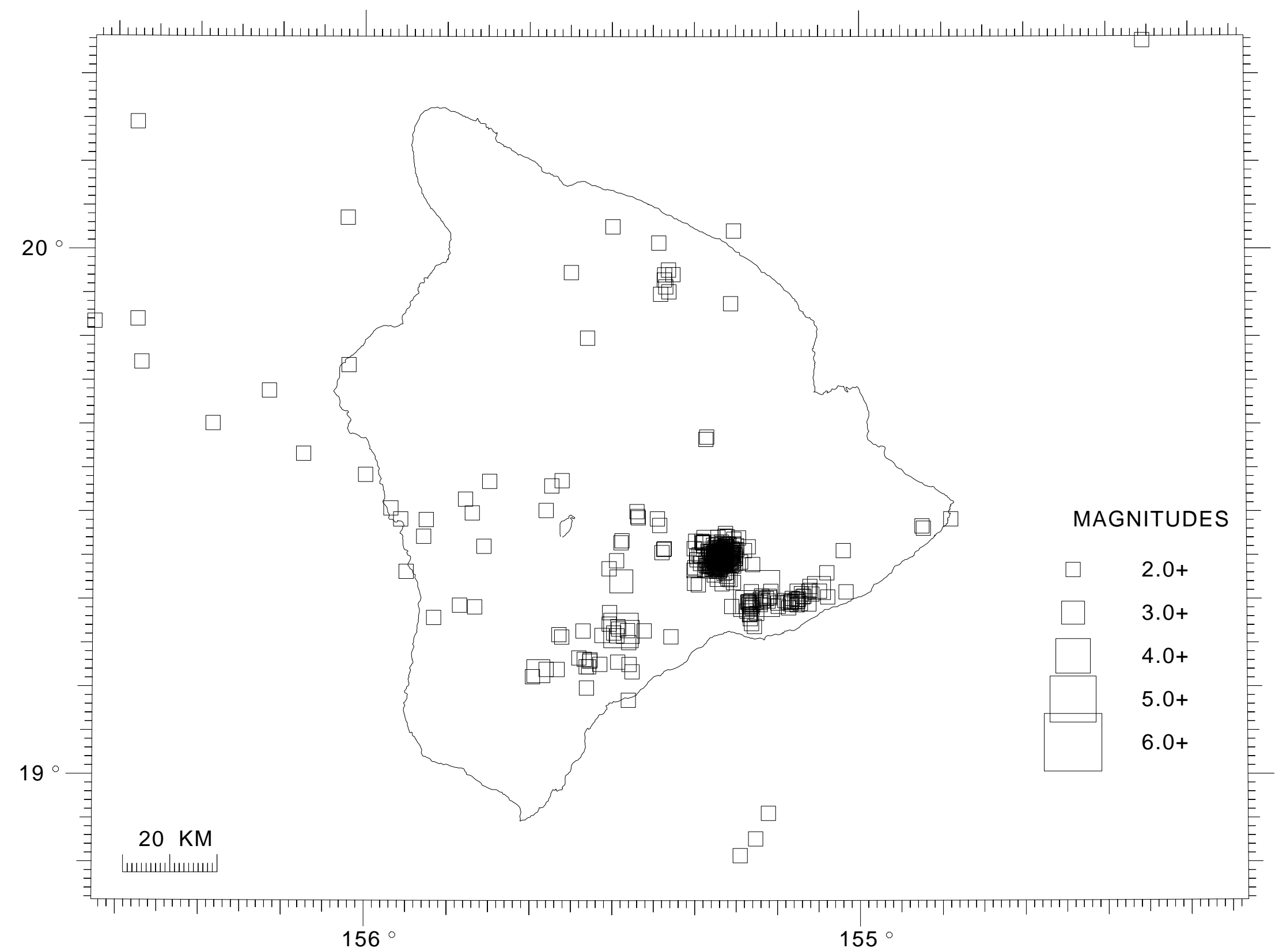


Figure 15. 2000 earthquake locations, Hawai'i Island, deep (13.1-60.0 km depth), $M>=2.0$.

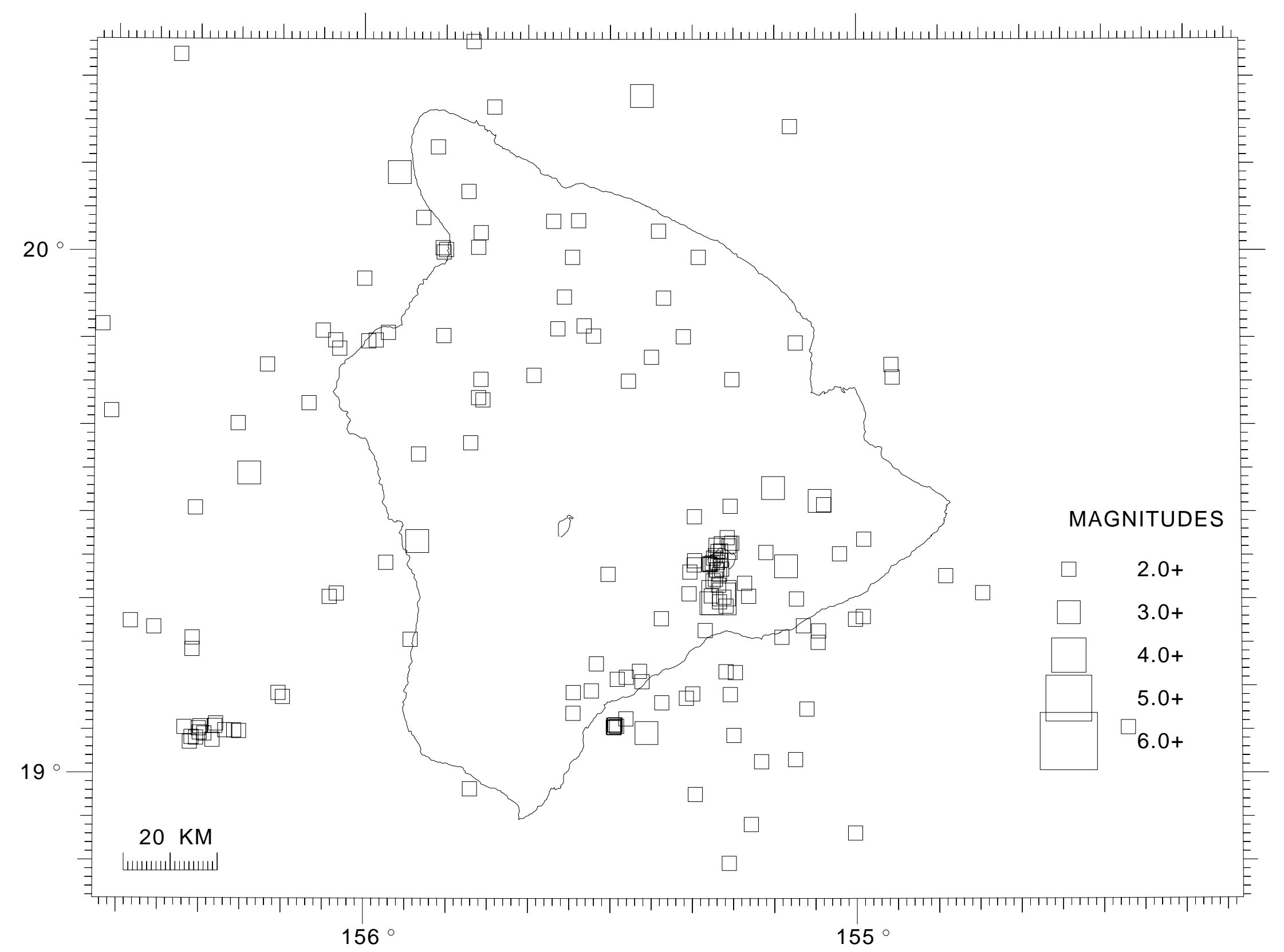


Figure 16. 2000 earthquake locations, Kilauea summit, shallow (0-5.0 km depth), $M>=1.0$.

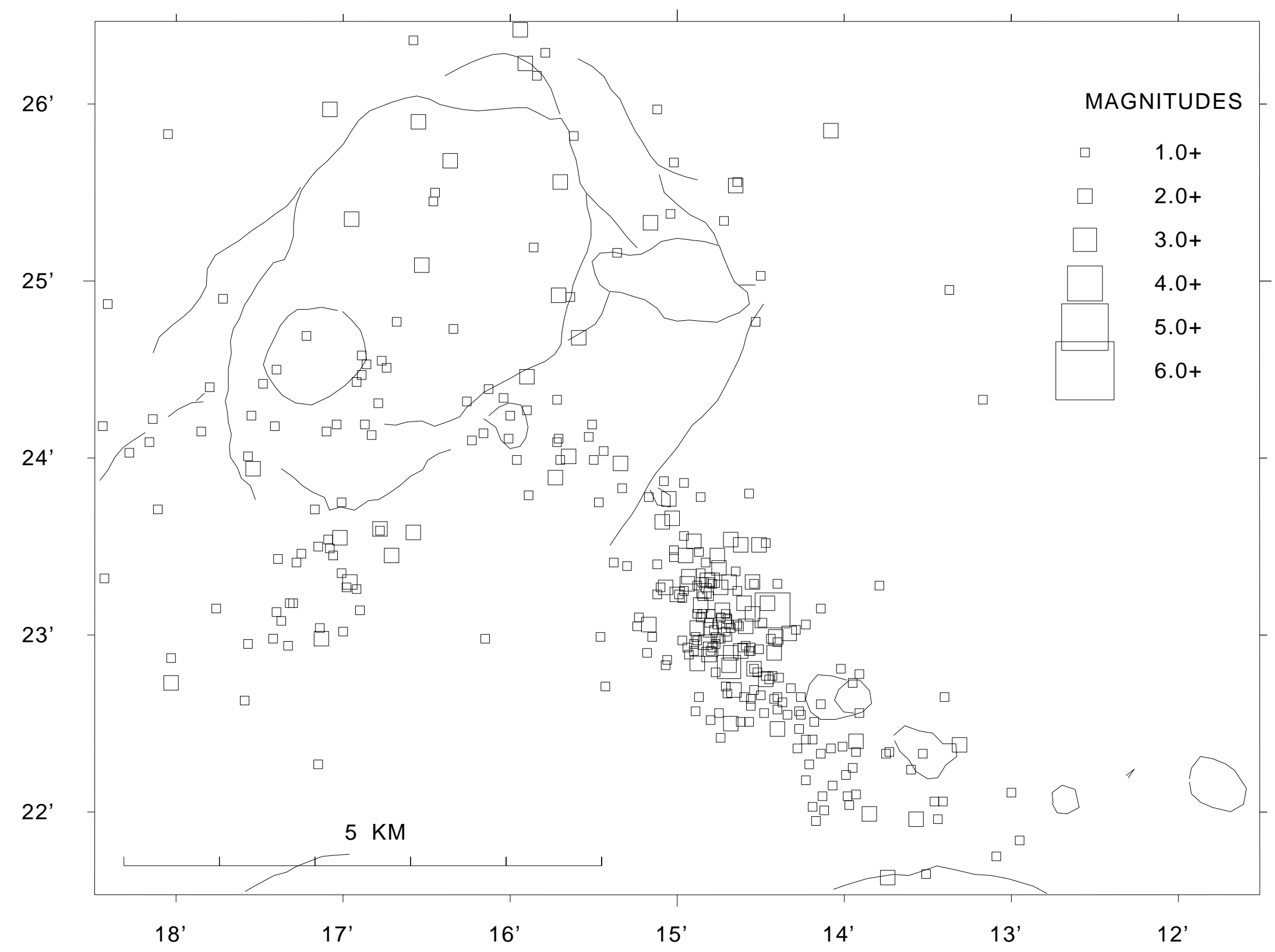


Figure 17. 2000 earthquake locations, Kilauea summit, intermediate $(5.1-13.0 \mathrm{~km}$ depth), $M>=1.0$.

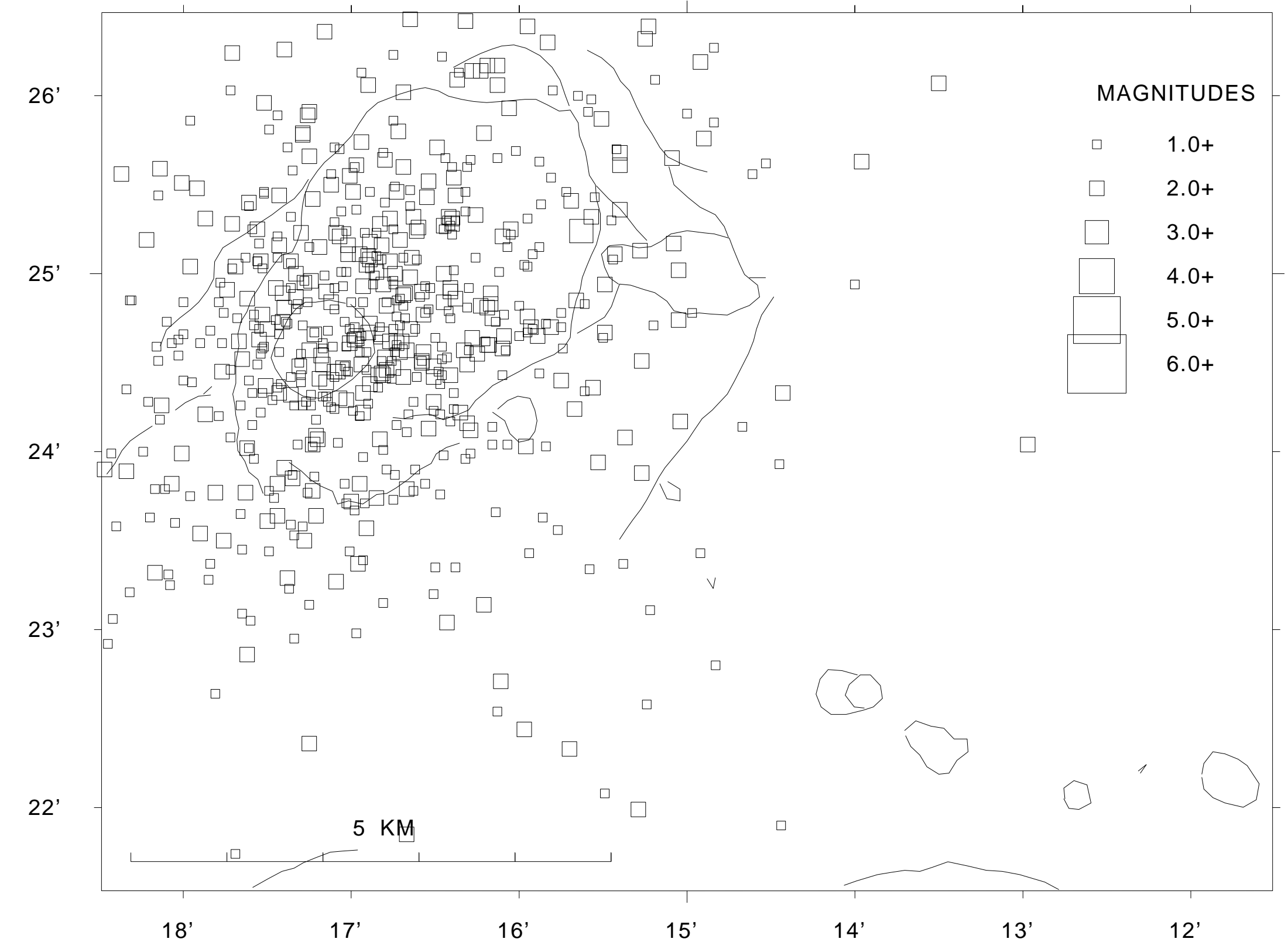


Figure 18. 2000 earthquake locations, Kilauea summit, deep (13.1-60.0 km depth), $M>=1.0$.

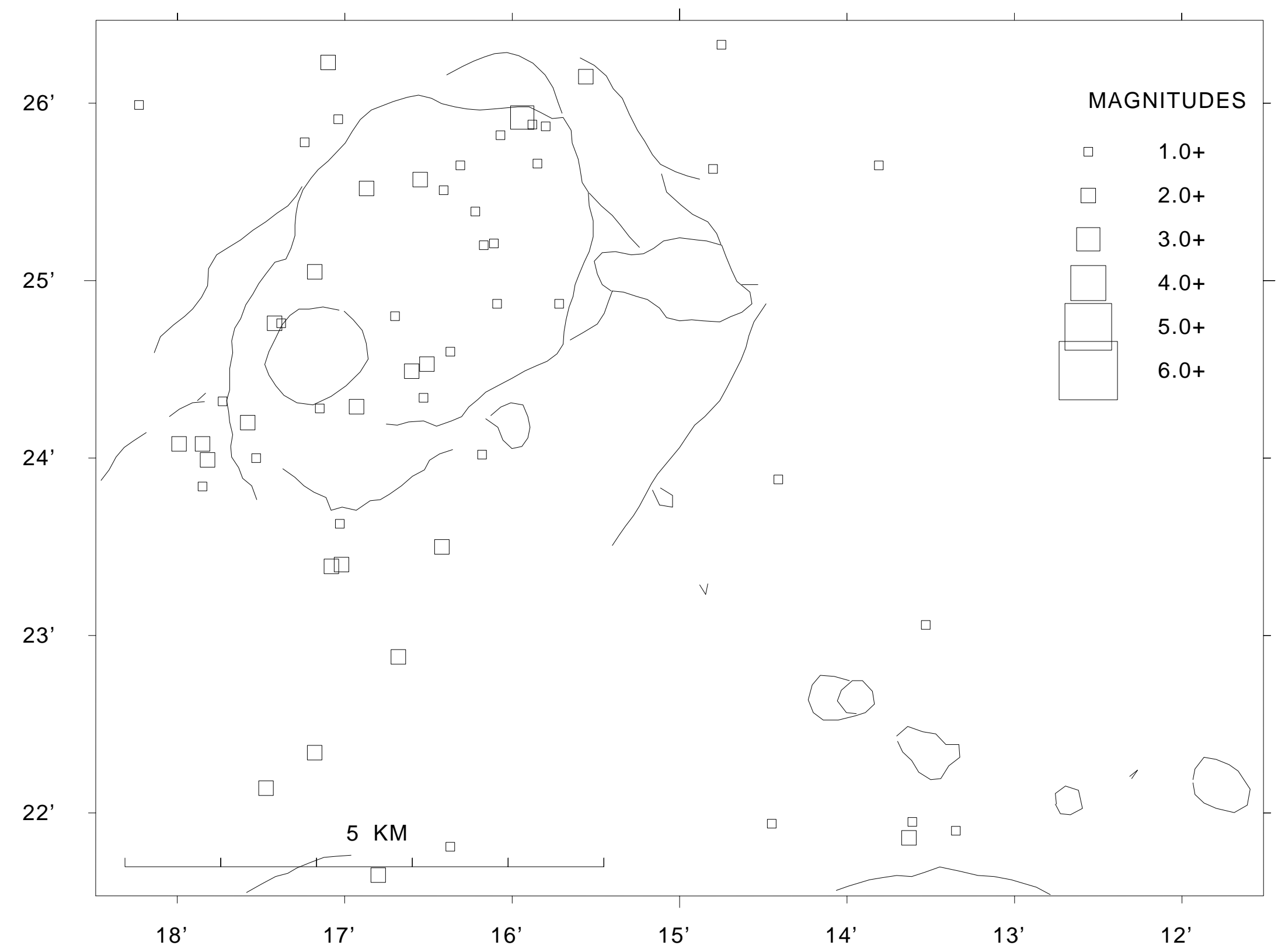


Figure 19. 2000 earthquake locations, Kilauea south flank, shallow $(0-5.0 \mathrm{~km}$ depth $), M>=2.0$.

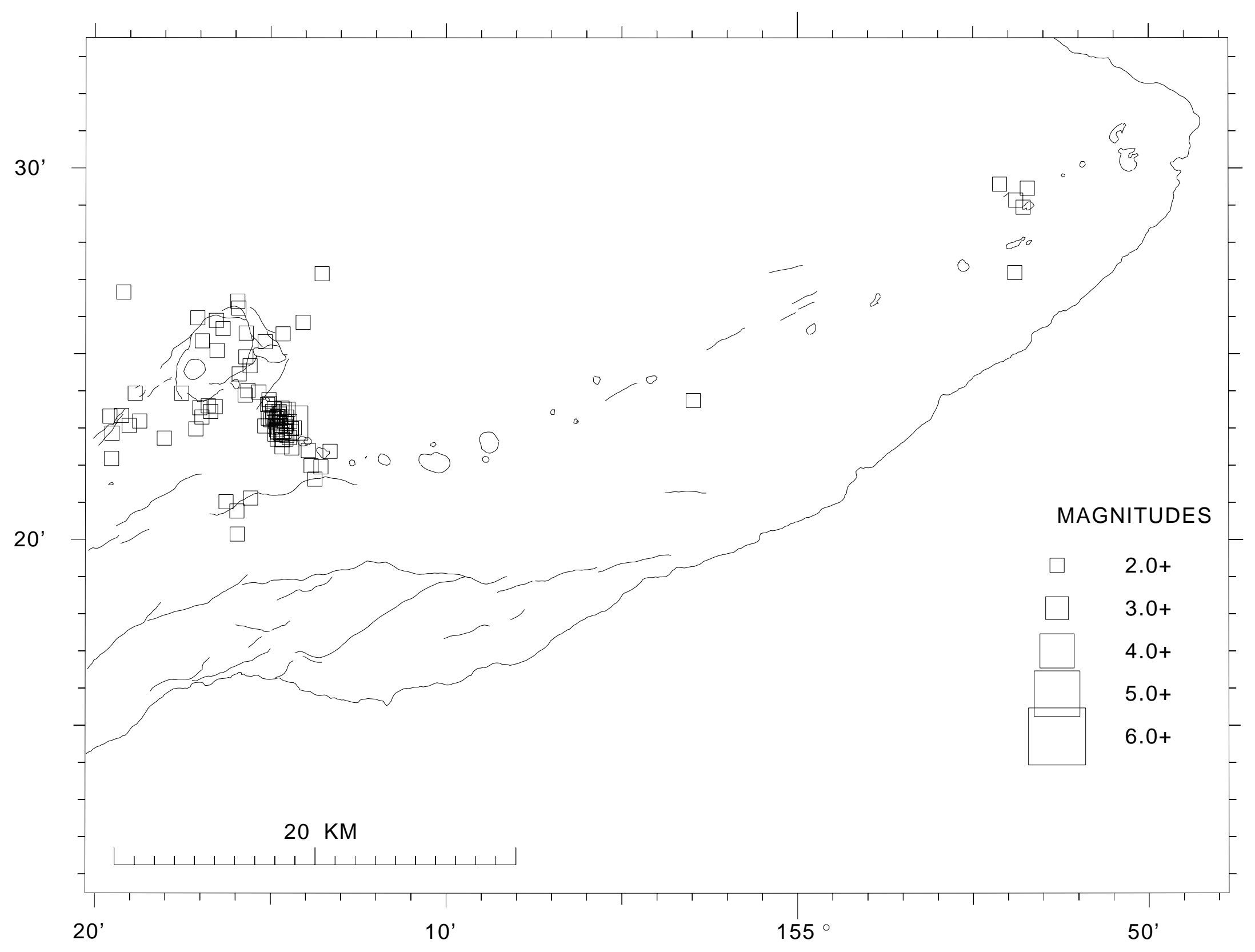


Figure 20. 2000 earthquake locations, Kilauea south flank, intermediate $(5.1-13.0 \mathrm{~km}$ depth), $M>=2.0$.

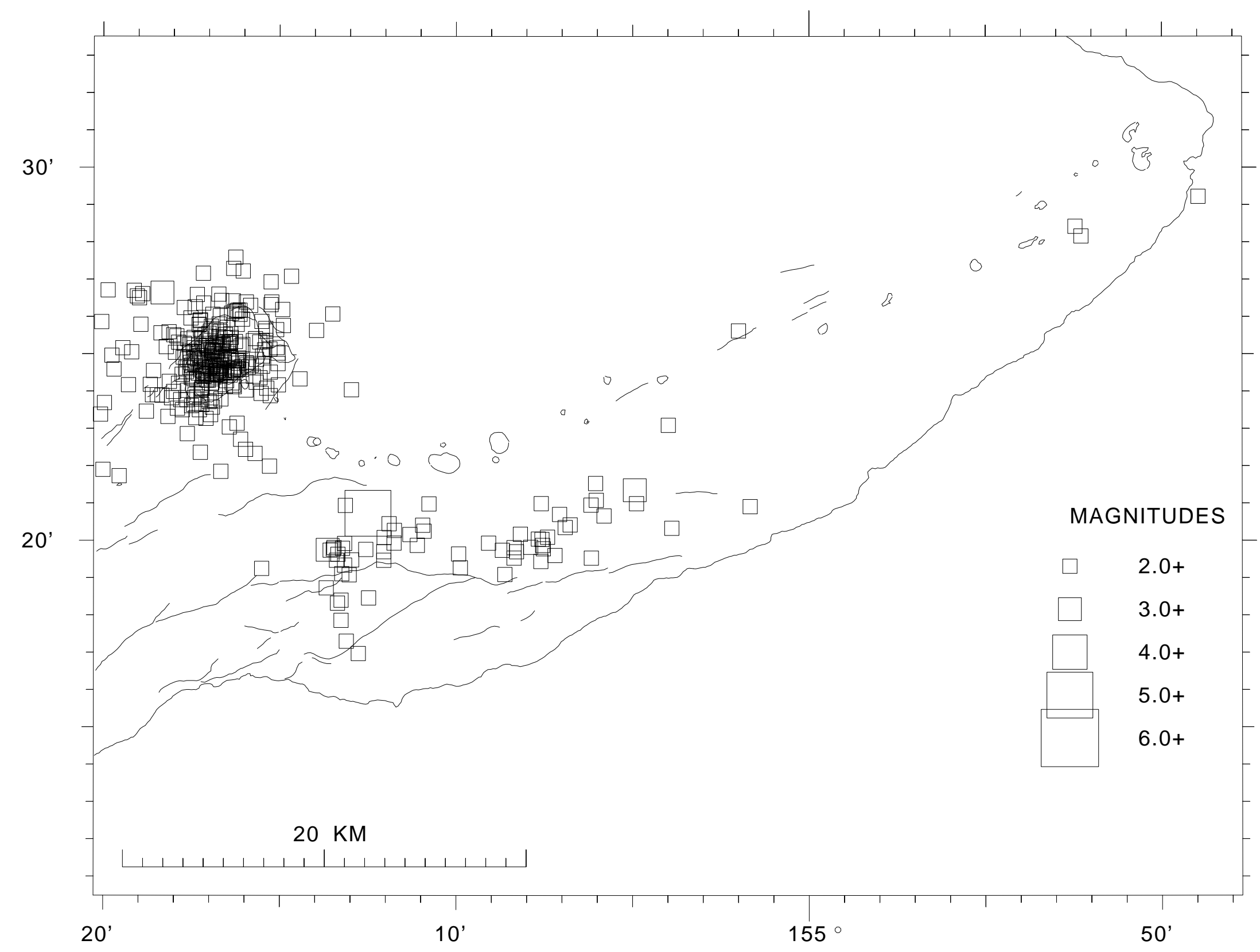


Figure 21. 2000 earthquake locations, Kilauea south flank, deep (13.1-60.0 km depth), $M>=2.0$.

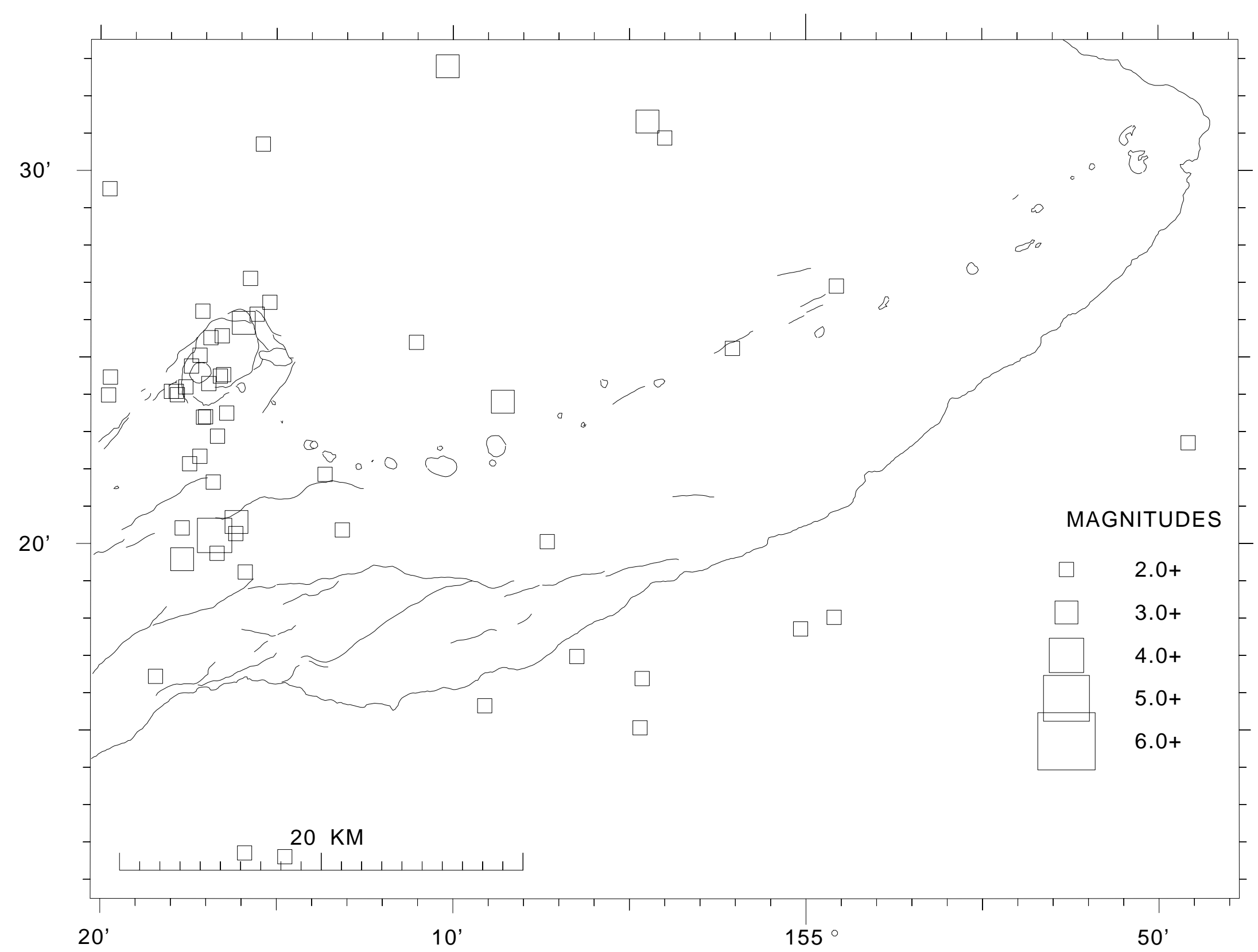


Figure 22. 2000 earthquake locations, Mauna Loa summit, shallow (0-5.0 km depth), $M>=2.0$.

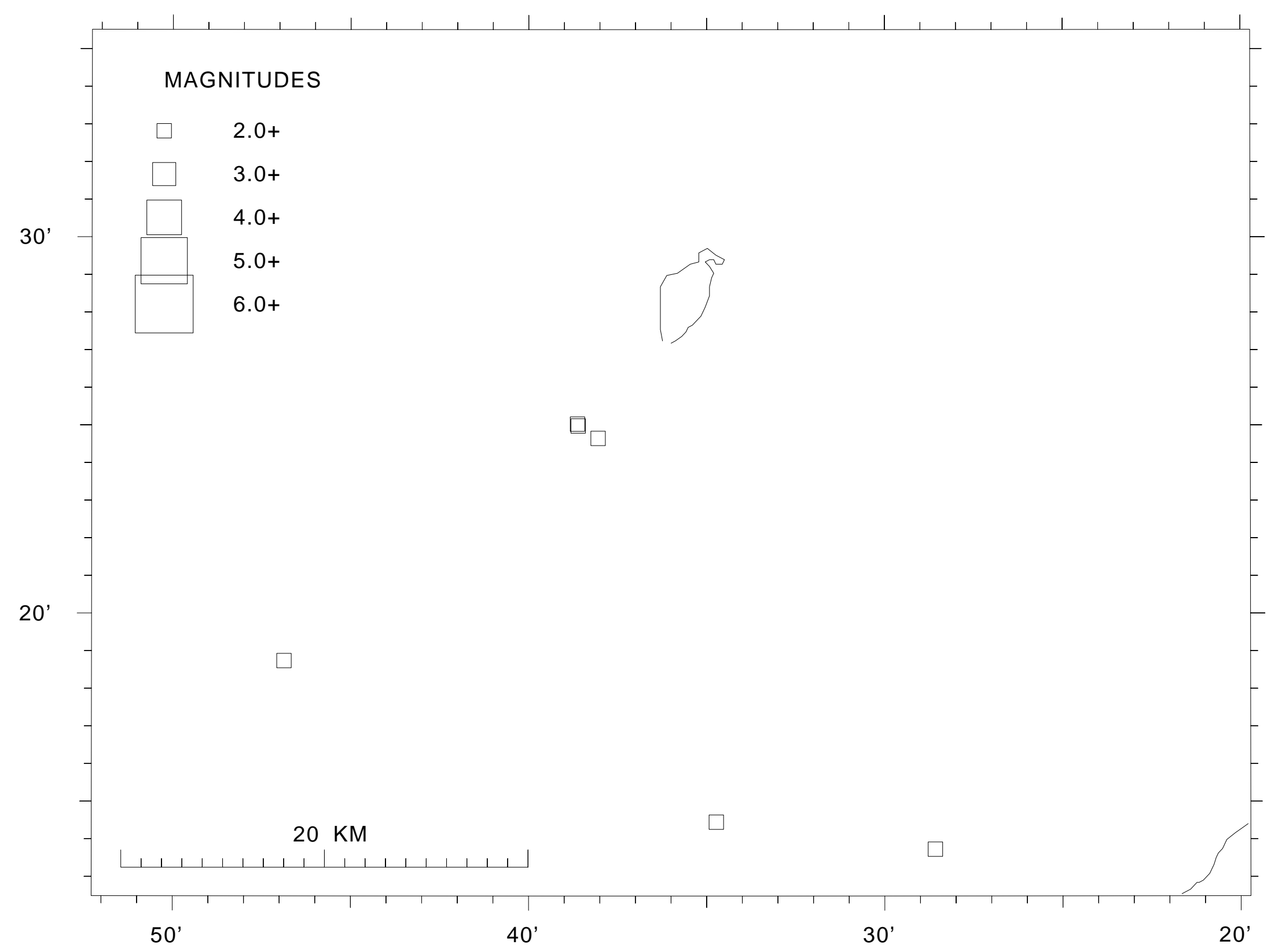


Figure 23. 2000 earthquake locations, Mauna Loa summit, intermediate $(5.1-13.0 \mathrm{~km}$ depth), $M>=2.0$.

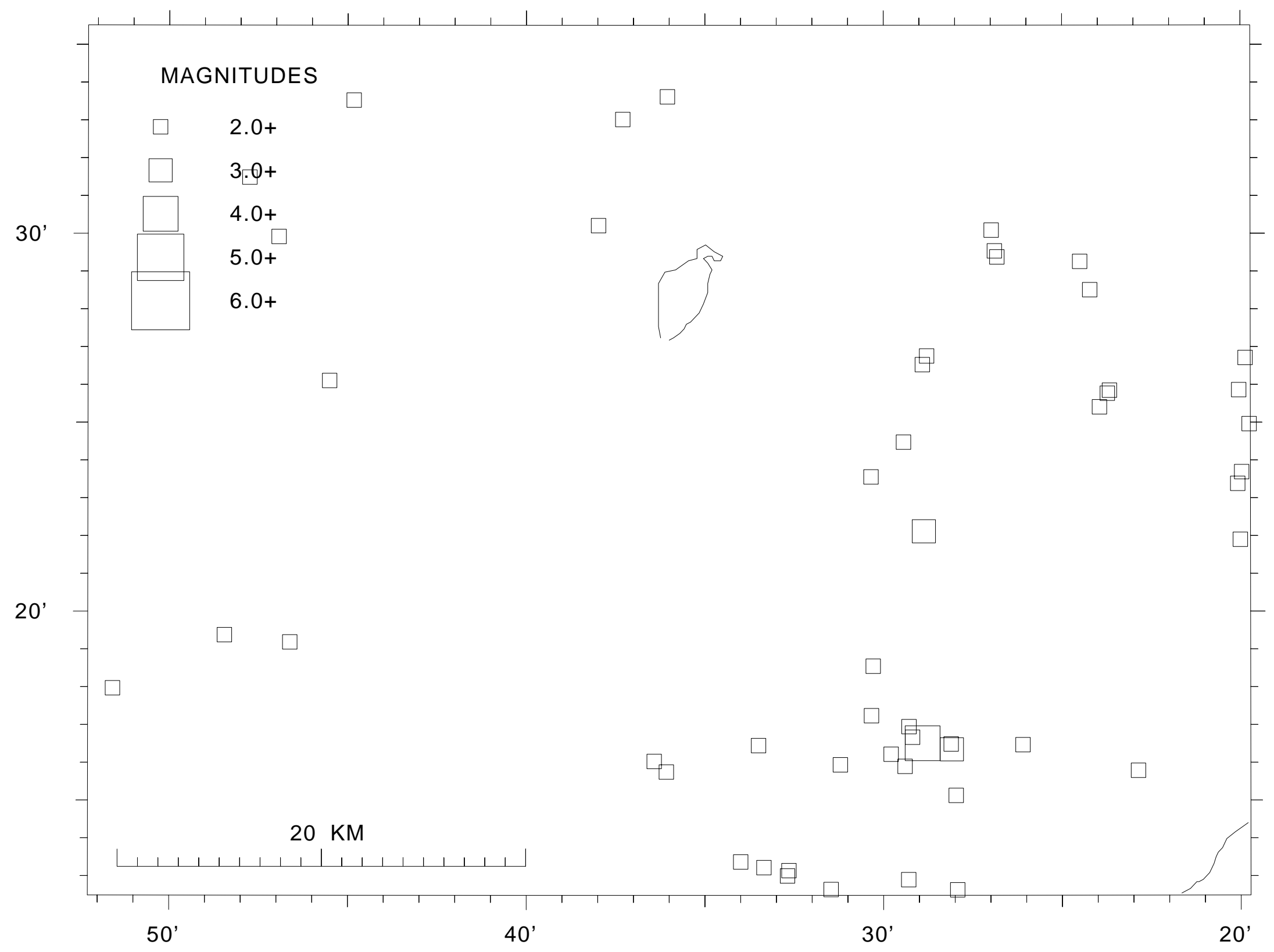


Figure 24. 2000 earthquake locations, Mauna Loa summit, deep $(13.1-60.0 \mathrm{~km}$ depth), $M>=2.0$.

\section{MAGNITUDES}

$2.0+$

$3.0+$

$4.0+$

$5.0+$

$6.0+$

20

$20 \mathrm{KM}$
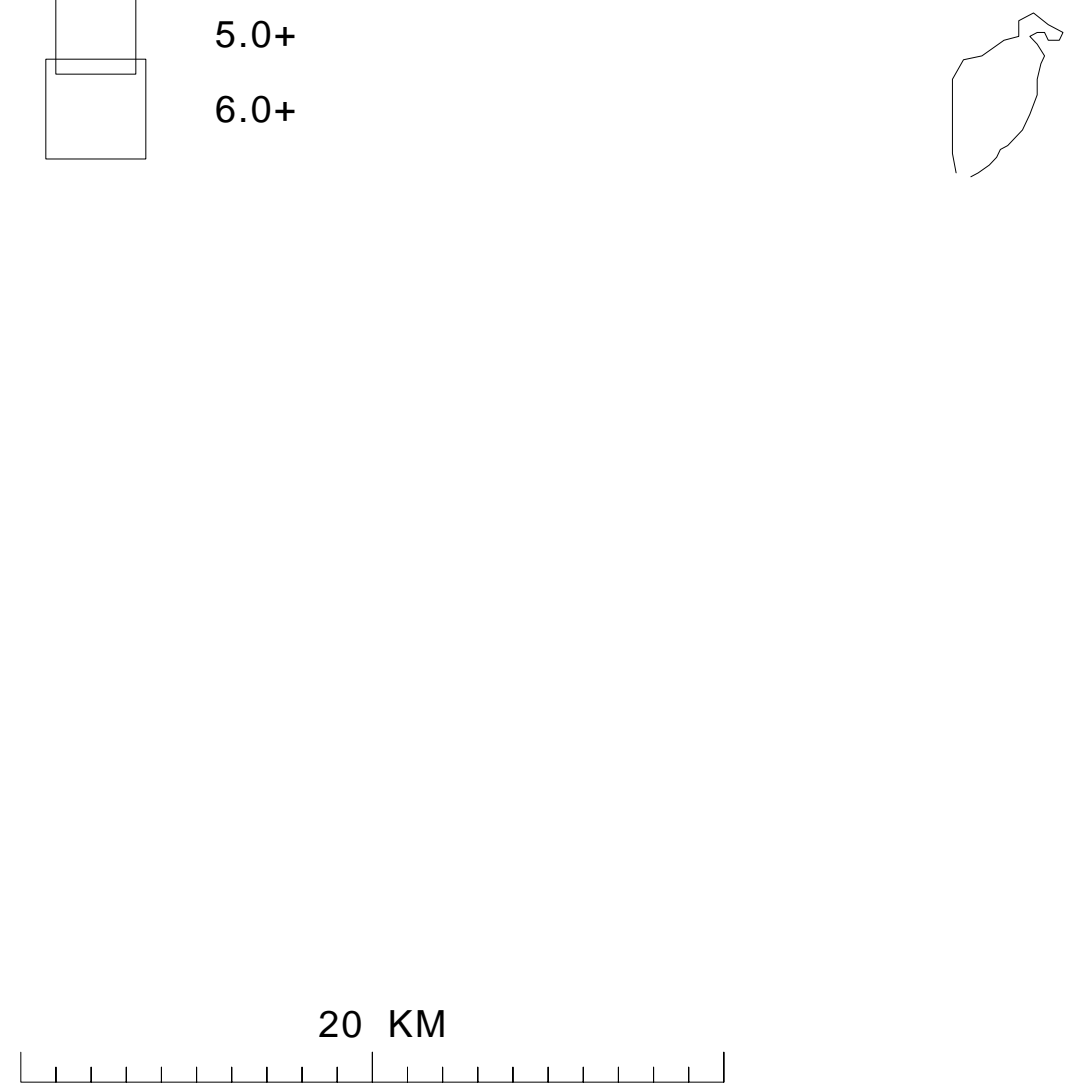

50 
Table 4 is a chronological list of selected events successfully located during 2000 . For each event, the following data are presented:

ORIGIN TIME - in Hawaiian Standard Time: date, hour (HR), minute (MN), and second (SEC).

EPICENTER - in degrees and minutes of north latitude (LAT N) and west longitude (LON W) in Old Hawaiian Datum.

DEPTH - Depth of focus in kilometers.

NRD - Number of $P \& S$ readings with final weights $>0.1$.

NS - Number of S readings with final weights $>0.1$

RMS SEC - Root mean square travel time residuals, in seconds.

ERH km - Standard error of the epicenter, in kilometers.

ERZ km - Standard error of depth of focus, in kilometers.

LOC REMKS - Remarks, three-letter code for geographic location of events. See Figures 7-10 for location of mnemonic code. Additional one-letter codes have the following meanings:

F felt

L long-period character

$\mathrm{T}$ associated with harmonic tremor

B quarry or other blast

\# the location program had a convergence problem, which usually means that the depth may be unreliable.

- $\quad$ the depth was held fixed.

PREF MAG - The preferred magnitude chosen from the available magnitudes.

Preference set as: X-amplitude magnitude, if none

D-Develocorder duration magnitude, if none

U-external magnitude, usually calculated from drum records.

NRD - The total weight of amplitude magnitude readings from contributing stations.

AZ GAP - Largest azimuthal gap in degrees between azimuthally adjacent stations.

MIN DS - Distance to the nearest station, in kilometers.

Table 5 is a list of events of magnitude 3.0 or greater, selected from Table 4 . 


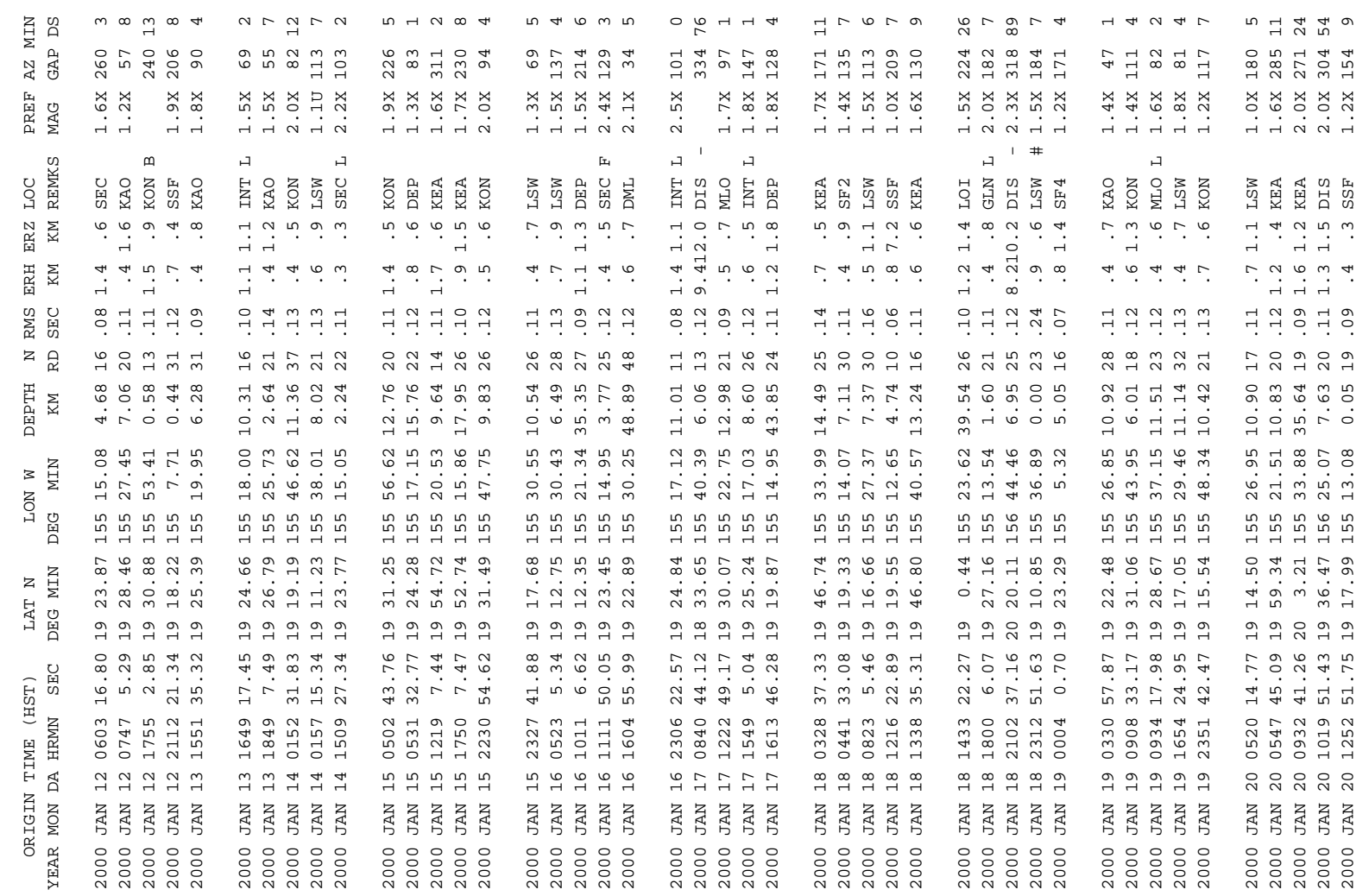

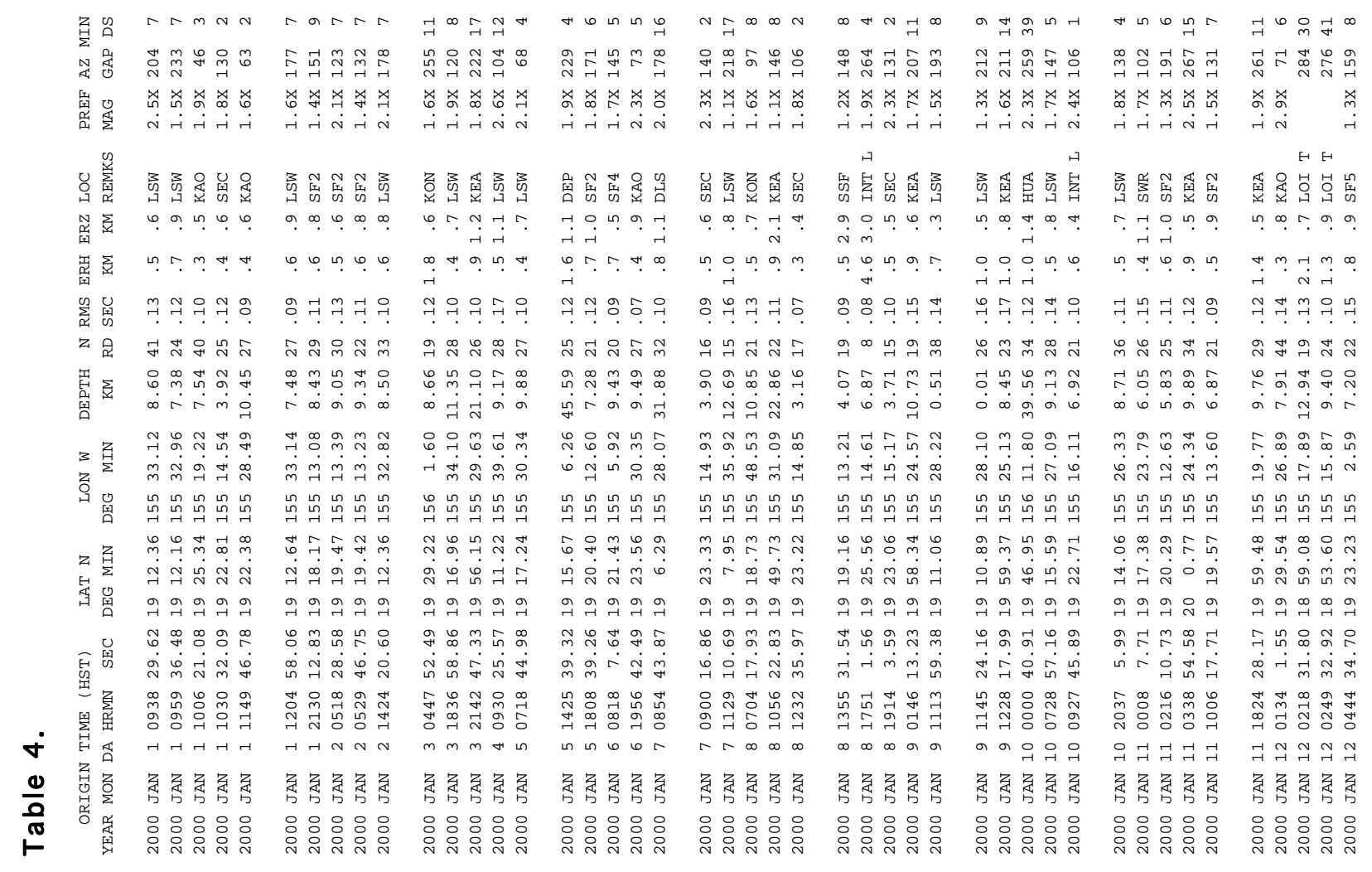




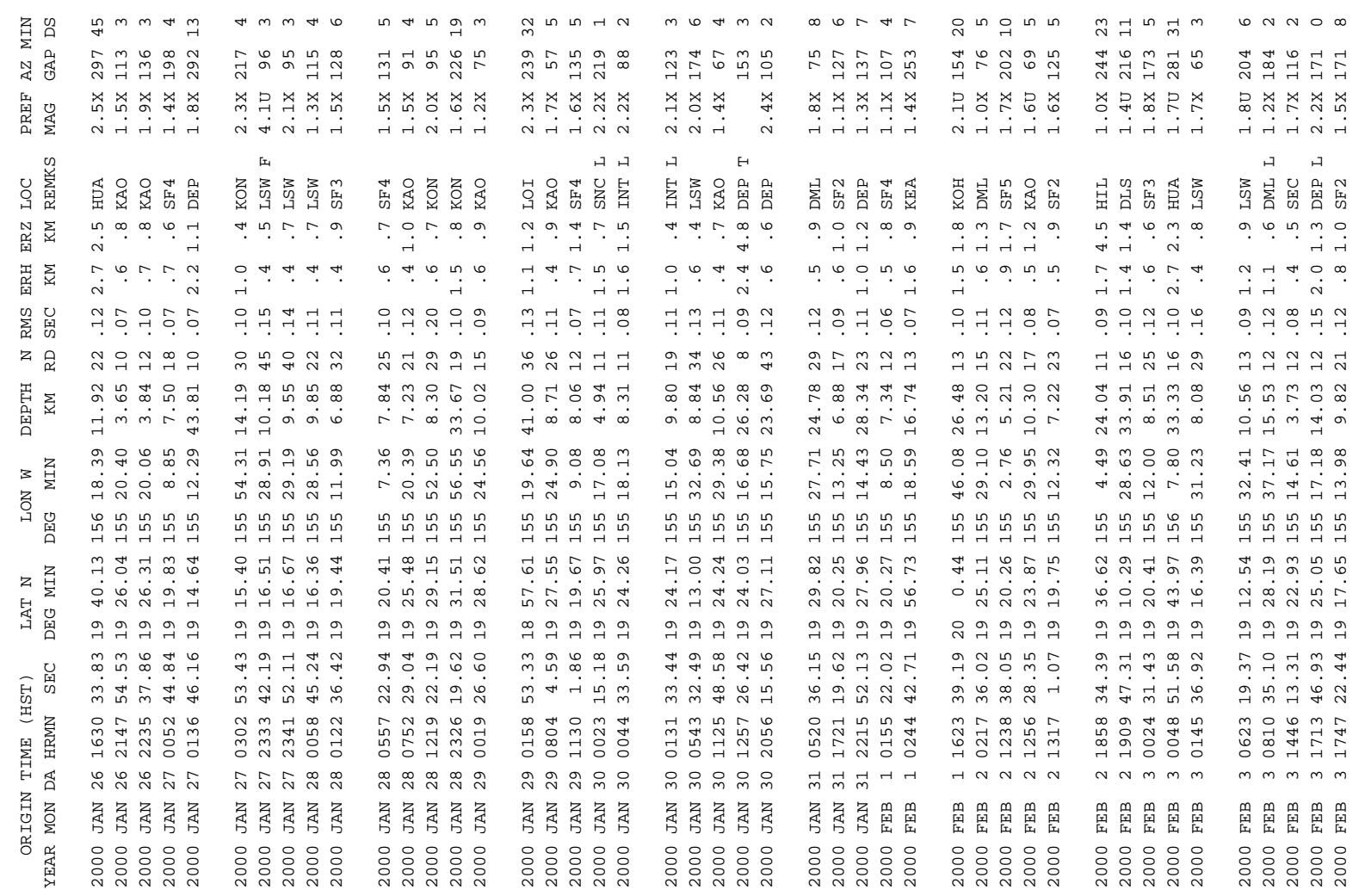

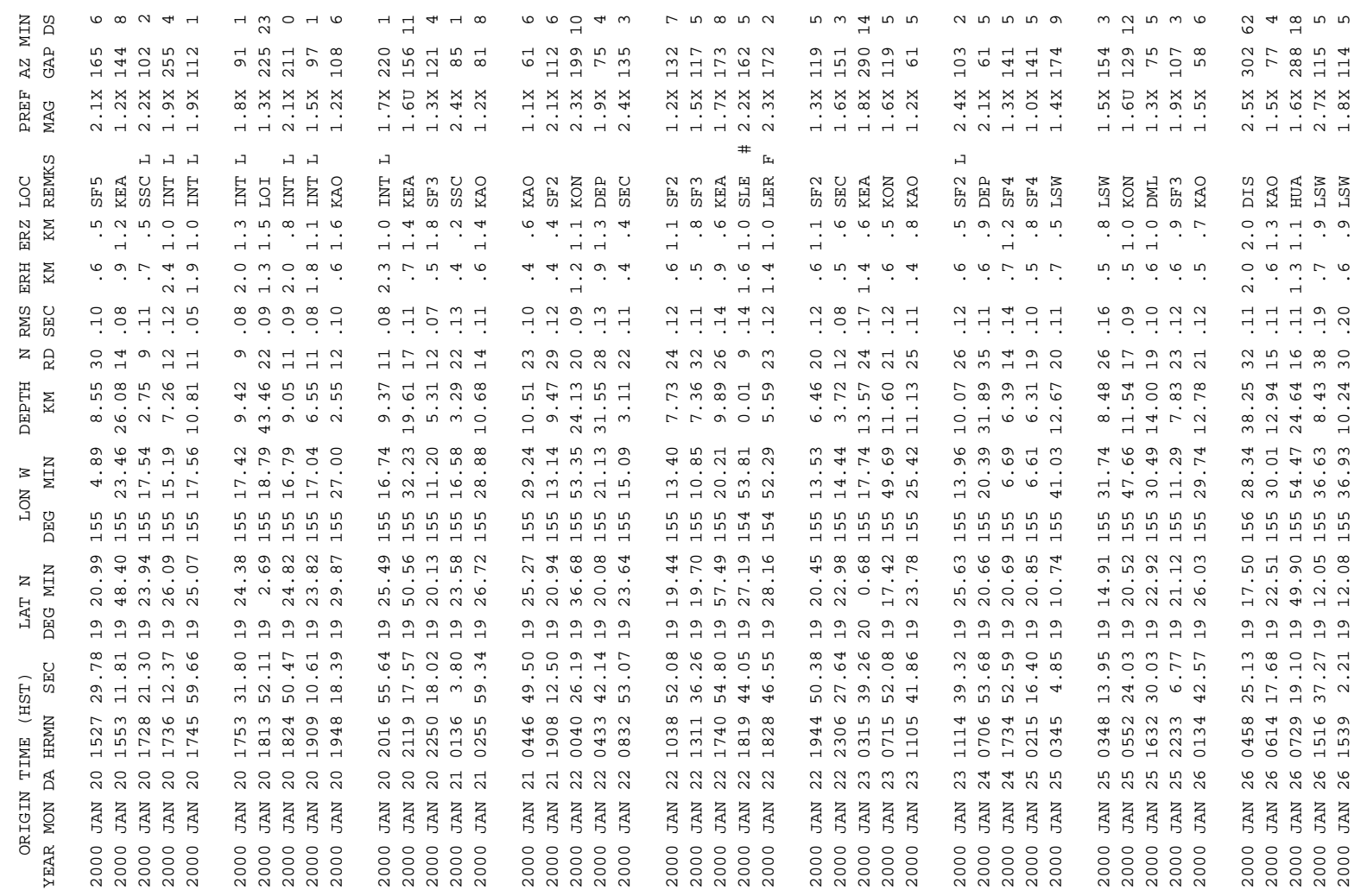




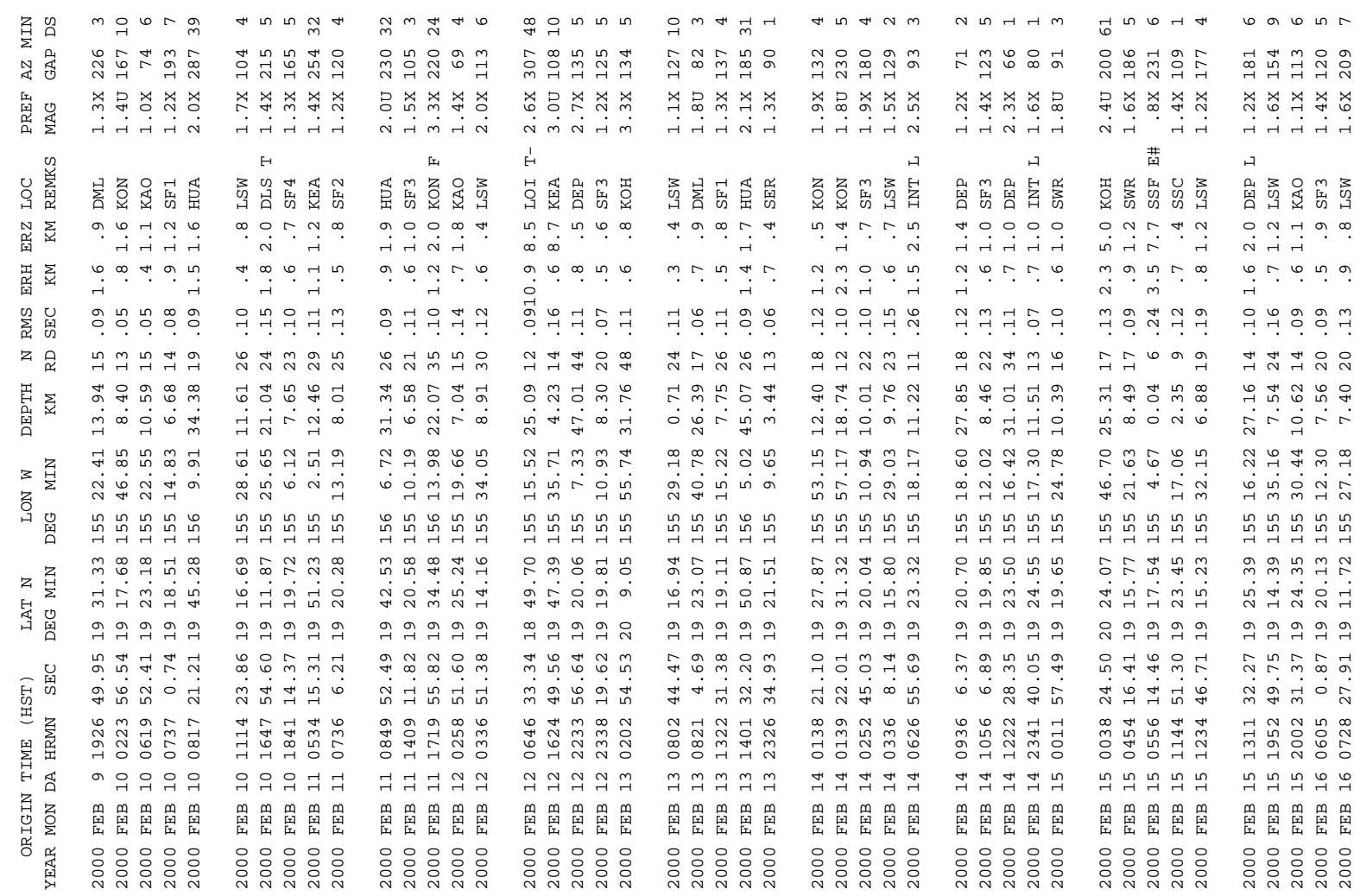

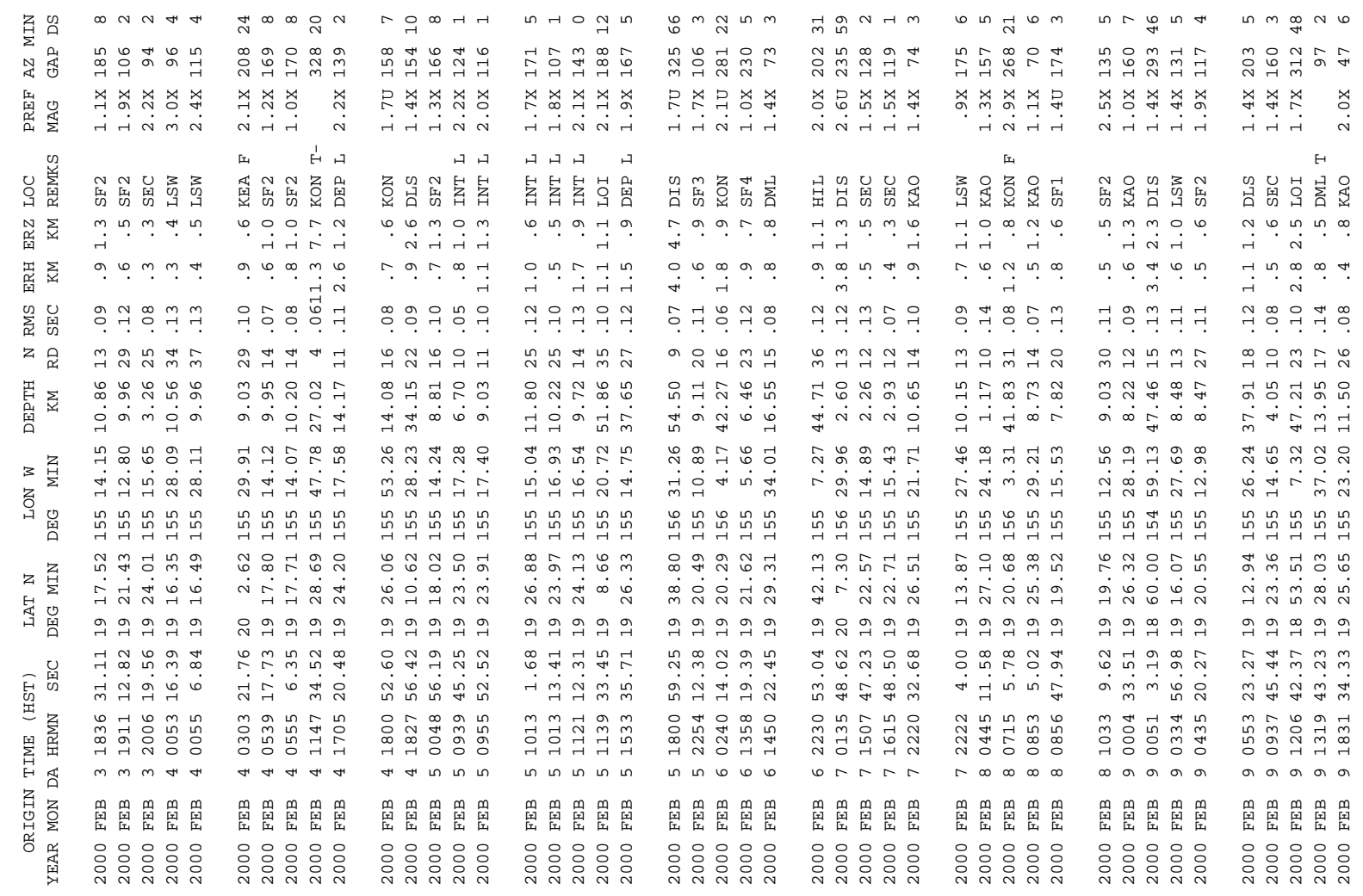




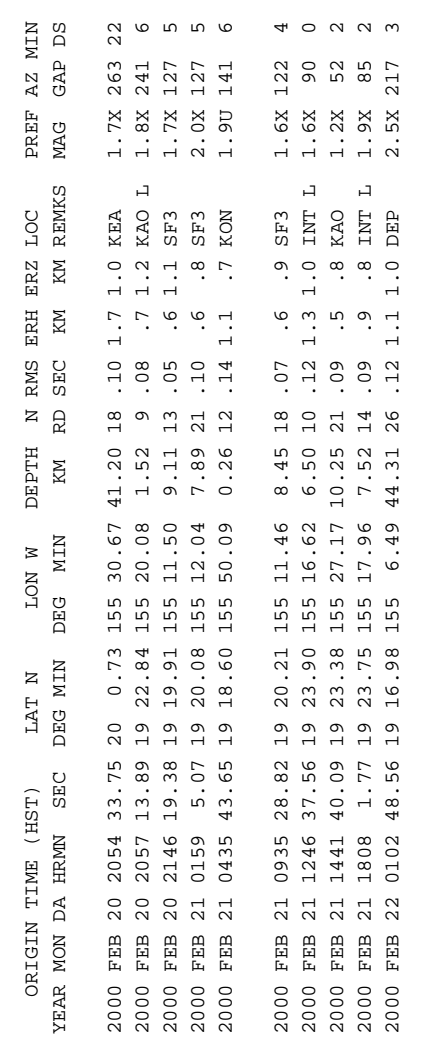

๓๐ ๘

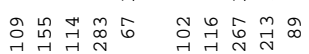

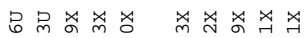

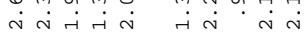

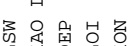

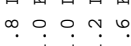
ํ..ㅇำ 국ำํำํํำ 이ำ ำก

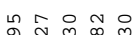

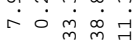

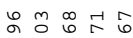
केंनें

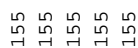
웅영우눈 जึ लं

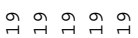

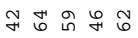
गं

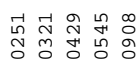
ก ก ก ก ก

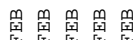
융유유

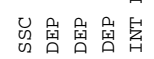
ㅁ.,$\infty . \circ 9$ ต.

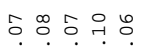

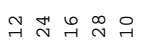

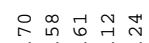

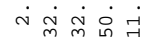

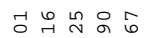

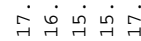

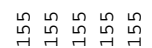
出会出둥

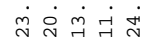

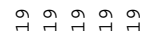

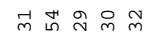

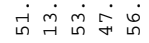

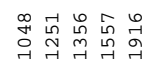
ก ก ก ก

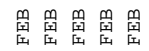
영: :
ㄴำ

希希希希赤 नंमेंत्र

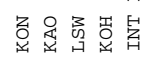
พ $9 . \circ \circ$

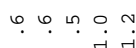

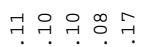

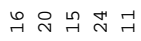
엉용용ㅁㅇ $\dot{\sim} \infty \dot{\sim} \dot{\sim} \dot{\omega}$

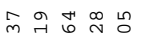

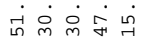

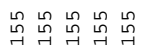

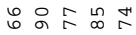
ปั่

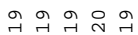
인 จं

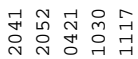
ำ $\mathbb{N} \underset{\sim}{ }$

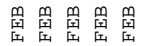

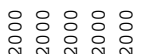

N $\mathrm{N}$ 呙 6 N

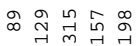

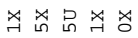
ज种的

바매

苗莦辟总界 नें

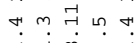
국ㄱㄱ웅ㅇㅇㅇㅇ 우궁

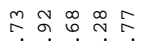
में

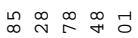
ते

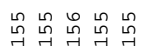
용

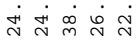
국그구 국ㅇ

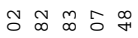
बें

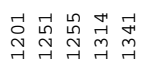

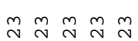

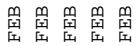
영: :

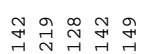

品希存希 मंनें

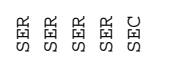

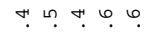
ษ.

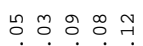

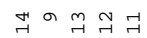
峞 $\dot{m} \dot{\sim} \dot{m} \dot{m} \dot{N}$

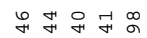

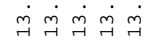
虽品嵒嵒品 웅용ㅇㅇㅇㅇㅇ

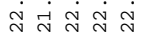

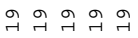
ํำ जं नें山े

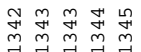

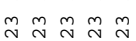

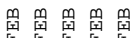
융ㅇㅇㅇㅇㅇ $\operatorname{dan} 2$

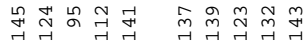

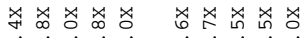

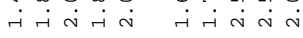

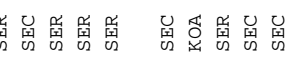

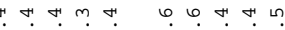

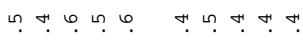

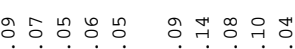

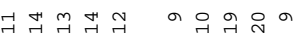

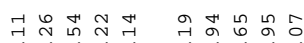

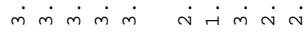

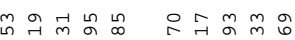

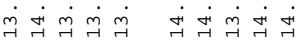

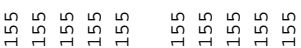

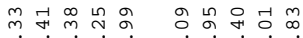
$\dot{\sim} \dot{\sim} \dot{\sim} \dot{\sim} \dot{\sim} \dot{\sim} \quad \dot{\sim} \dot{\sim} \dot{\sim} \dot{\sim} \stackrel{\sim}{\sim} \dot{\sim}$

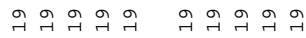
यू नें

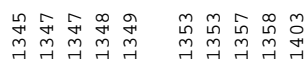

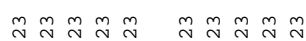

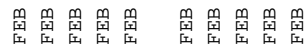

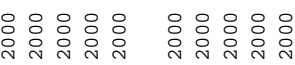

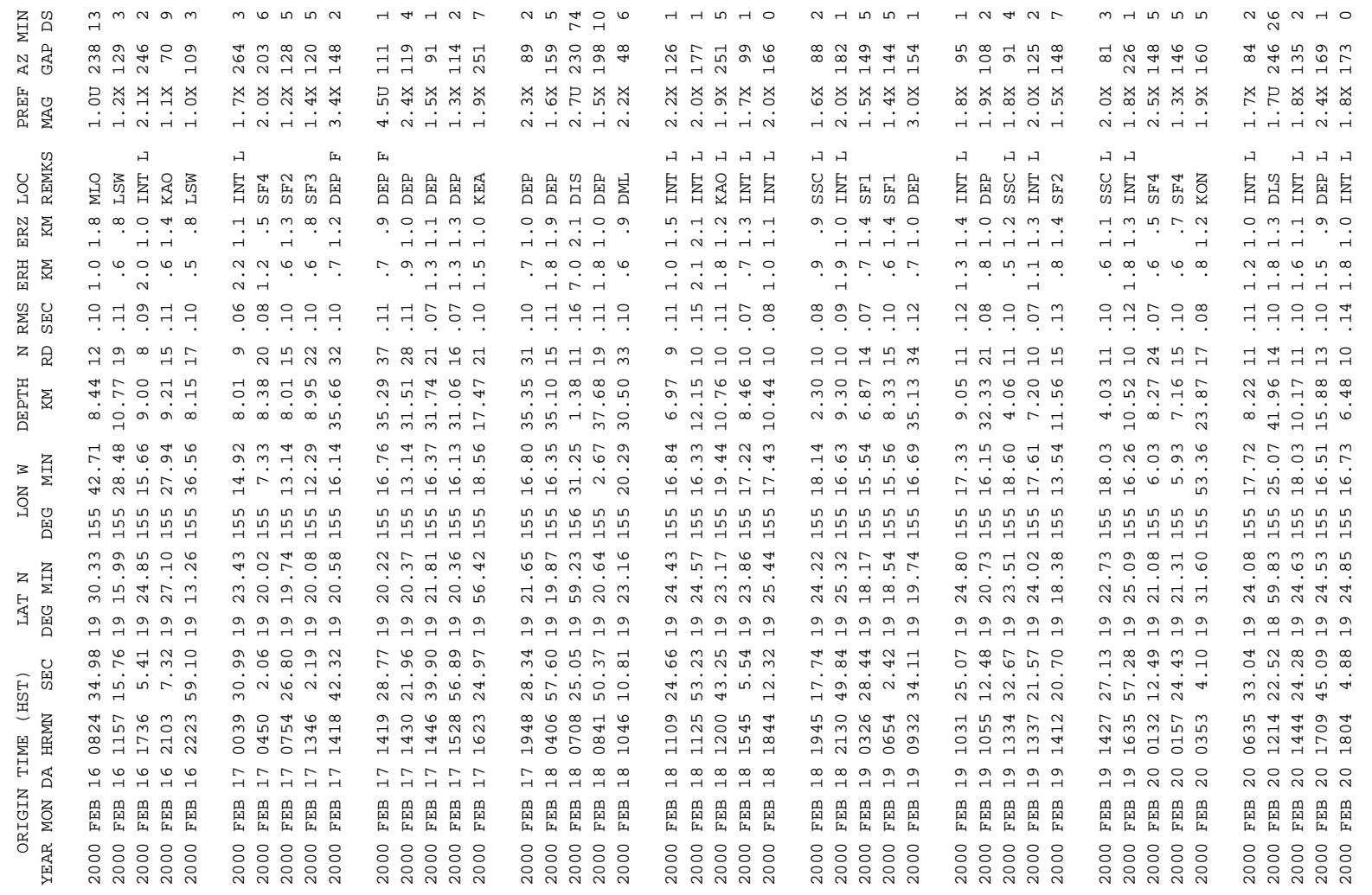




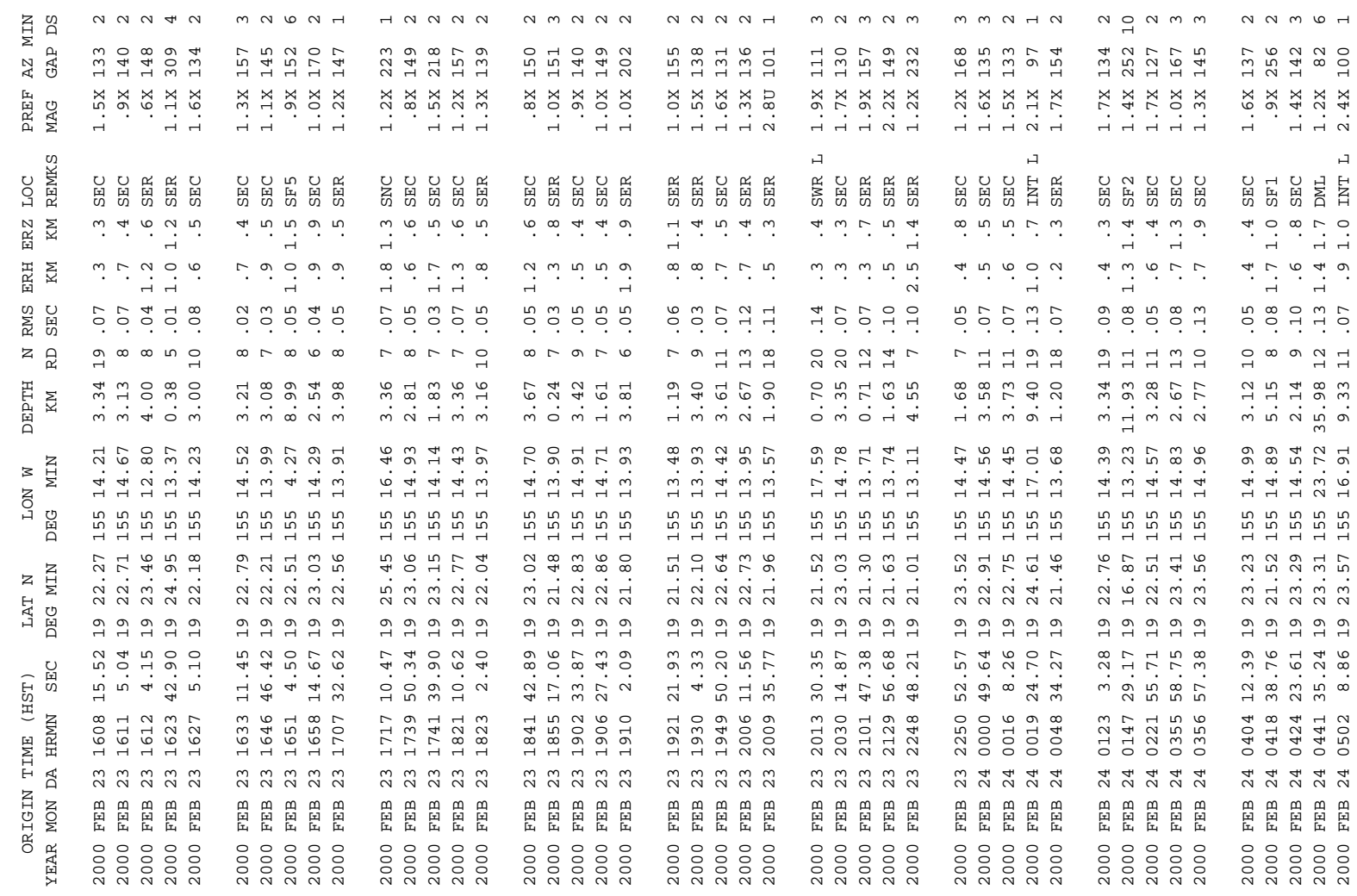

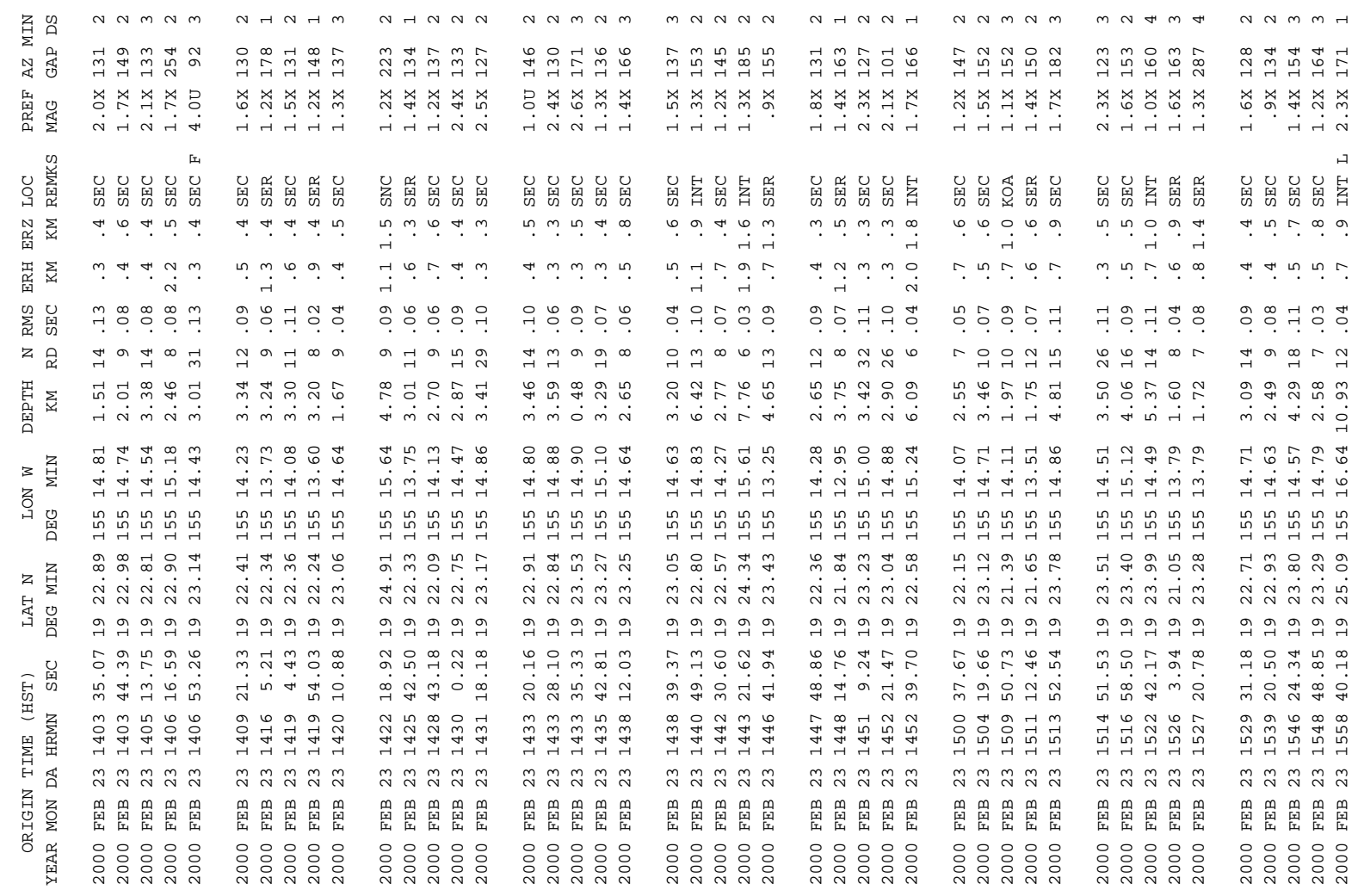




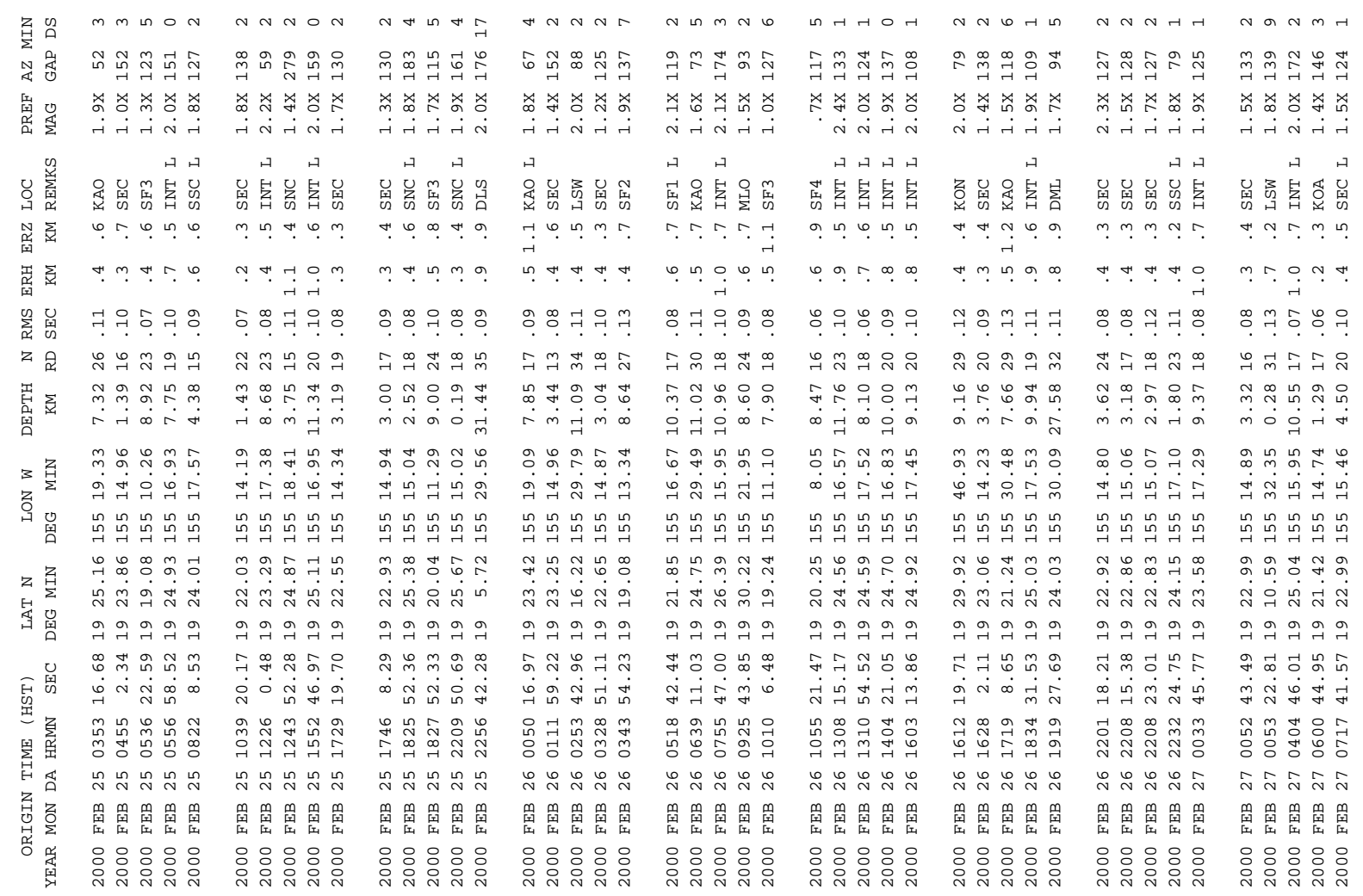

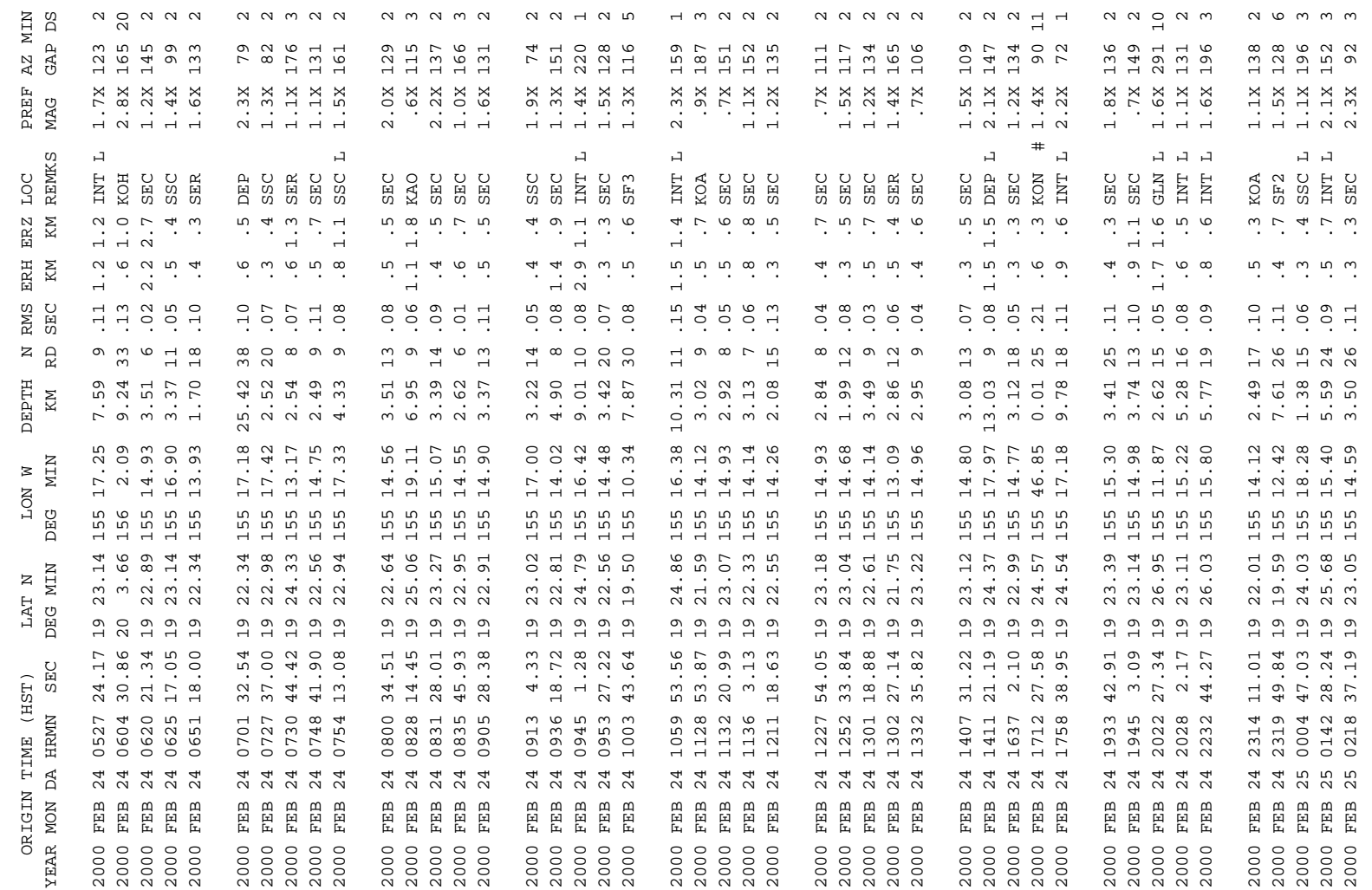




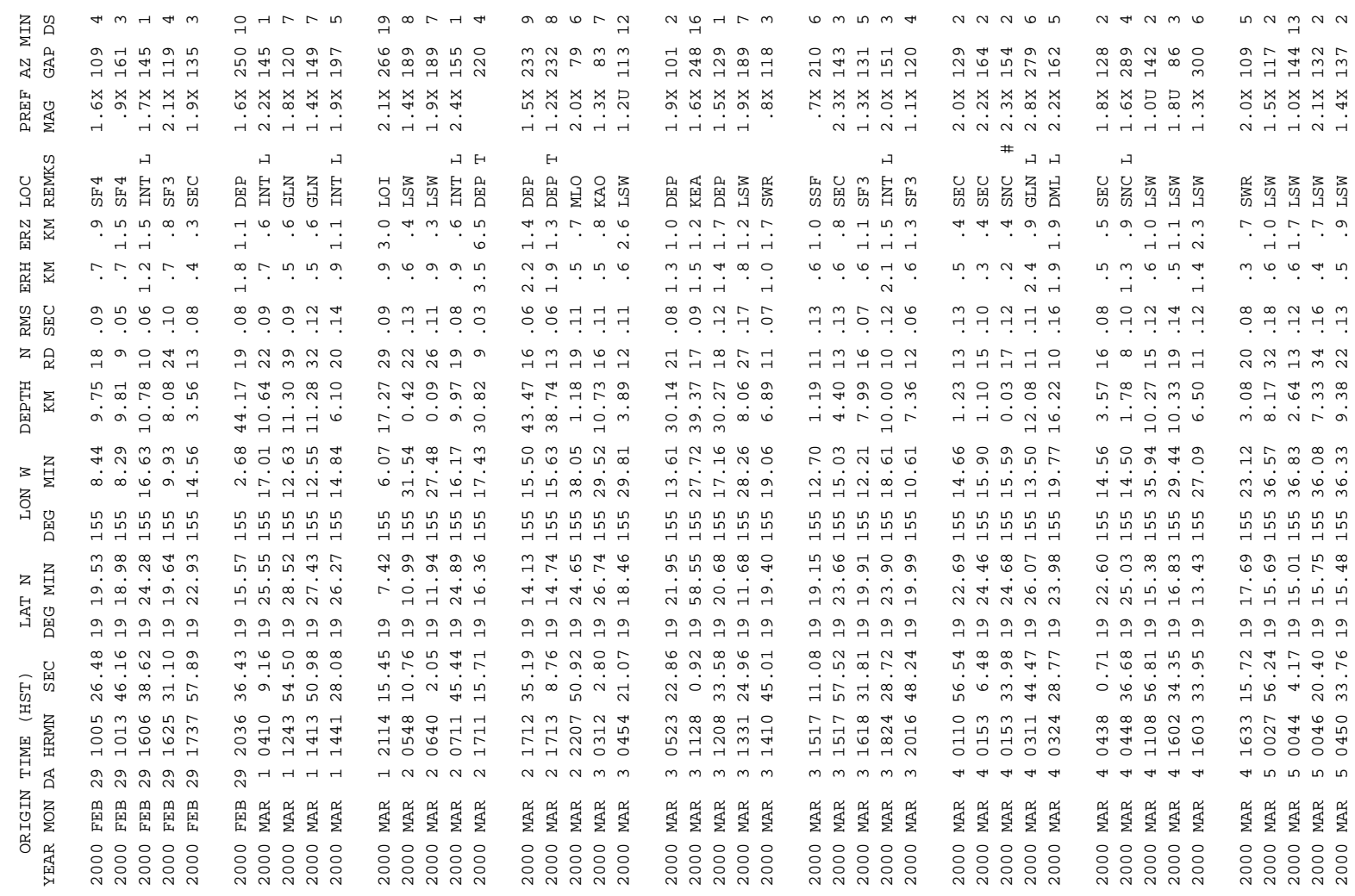

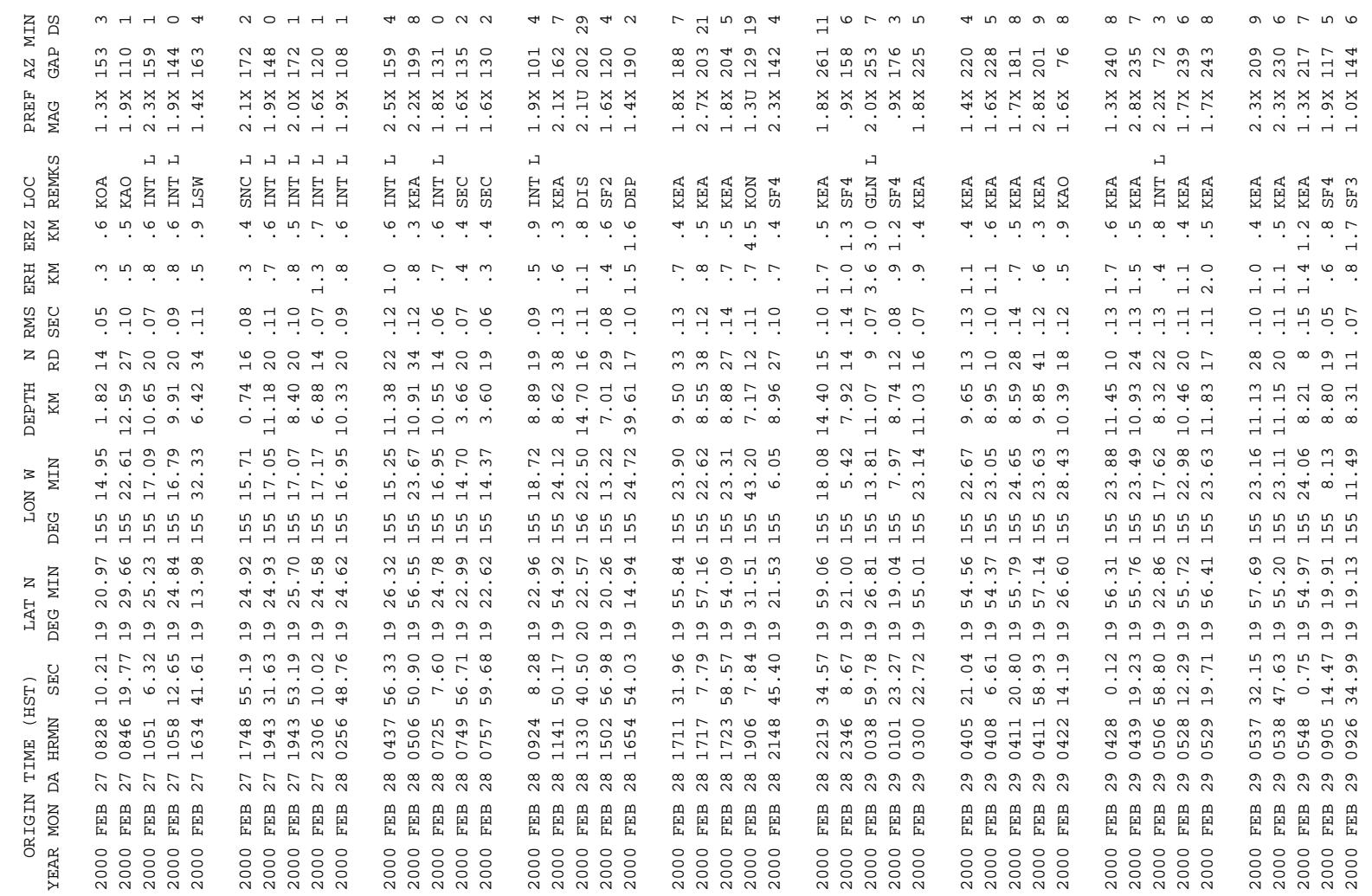




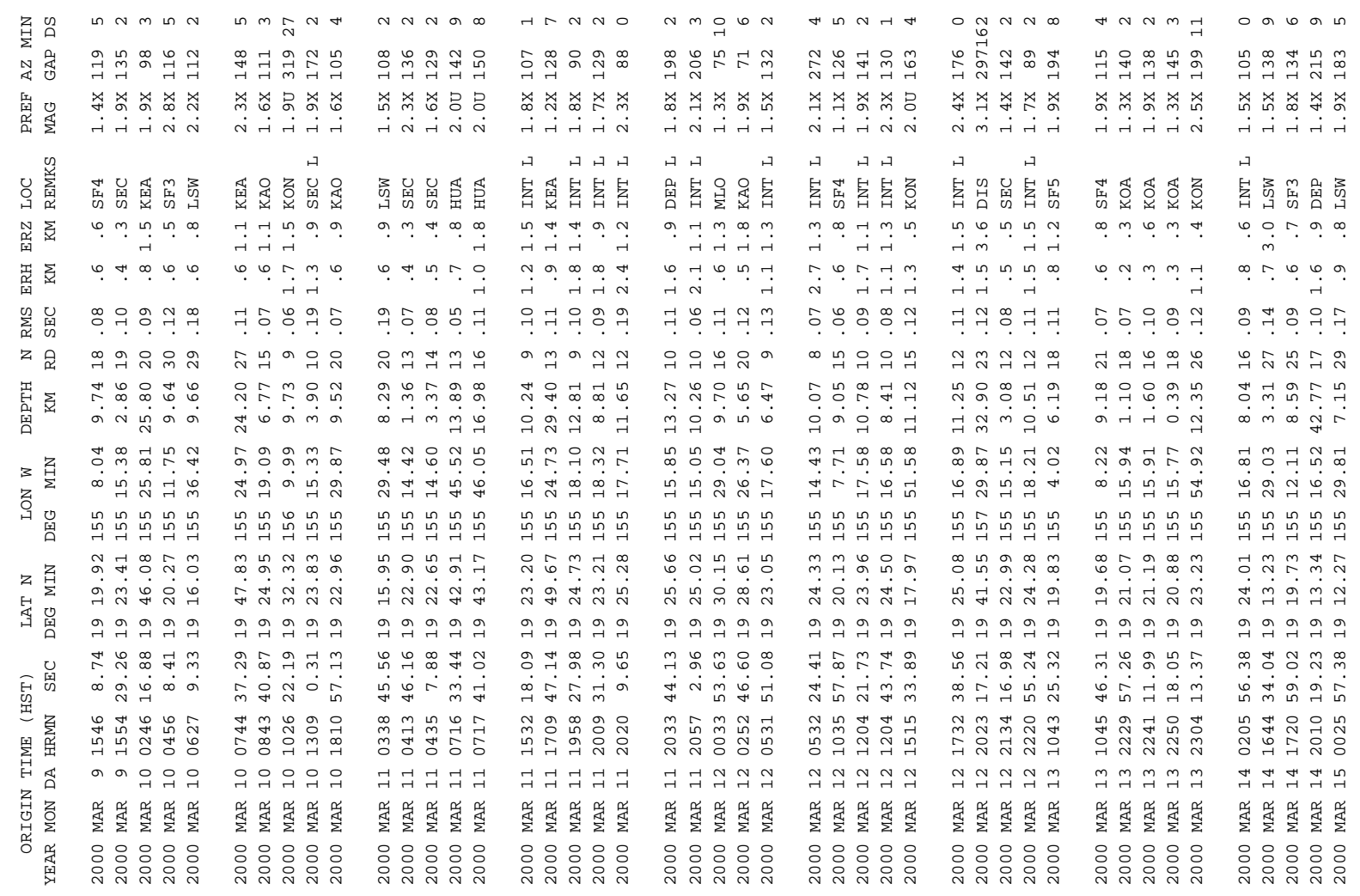

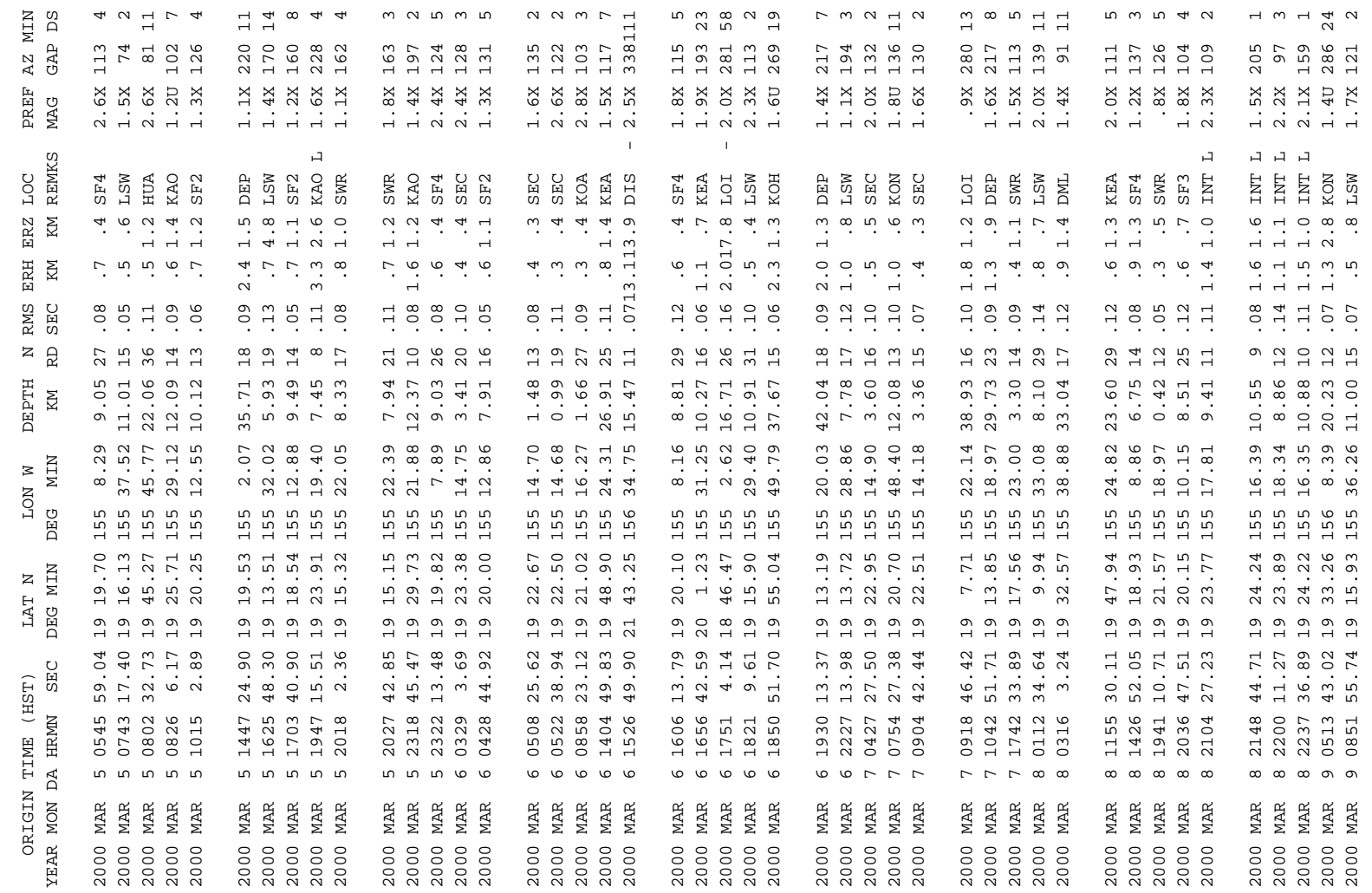




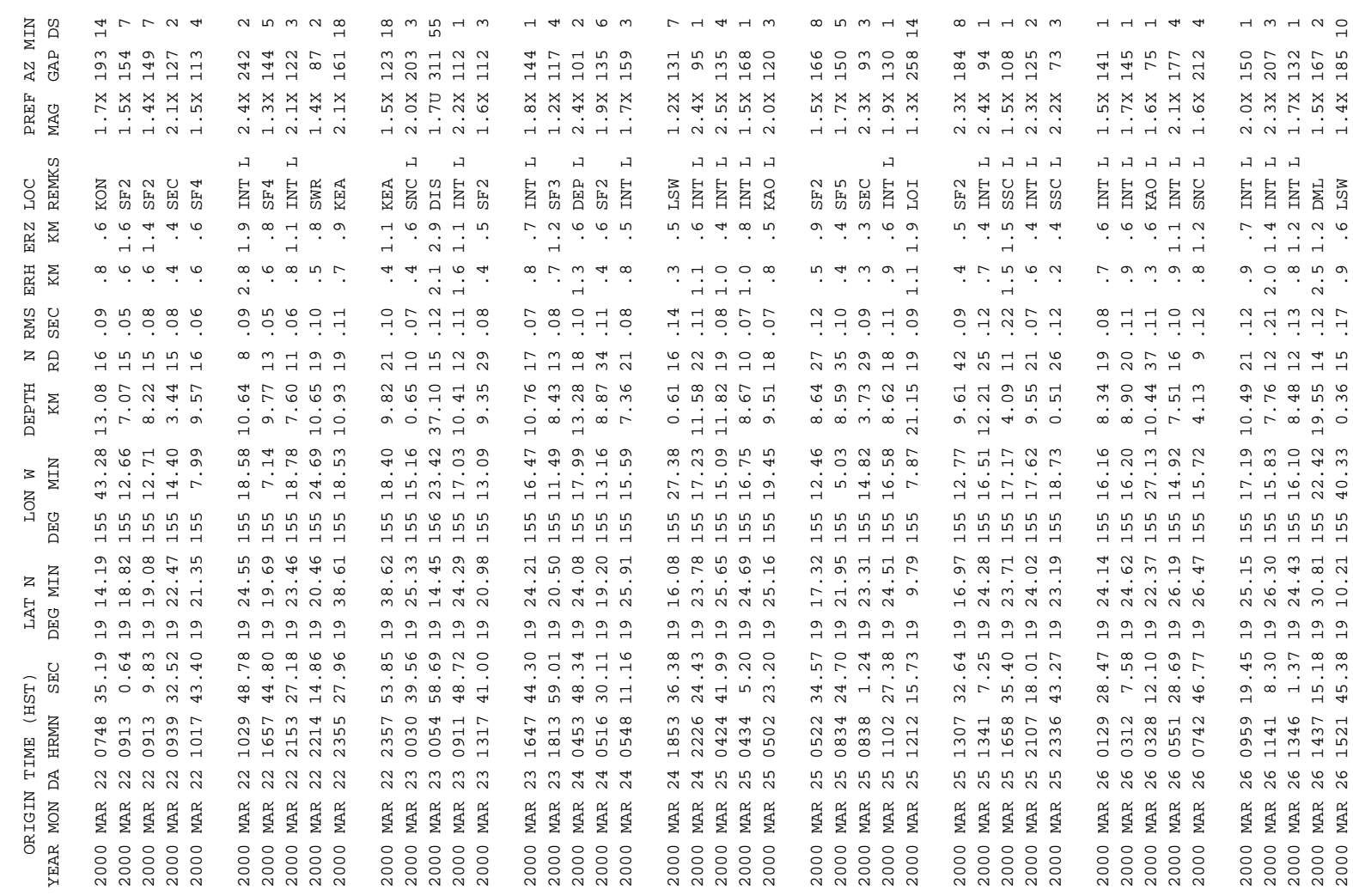

\begin{tabular}{|c|c|c|c|c|c|c|c|c|}
\hline $0 \underset{\sim}{0}$ ○ & $\sim \sim \underset{\neg}{*} \sim$ & $\curvearrowleft \exists ન r m$ & 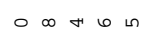 & $\cap \sim \curvearrowleft \curvearrowleft r$ & $6 m \neg N \infty$ & $\forall m \infty r$ 욱 & $\curvearrowleft \neg \infty \sim n$ & $\exists 00$ \\
\hline Nָ & 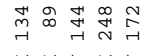 & 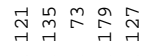 & 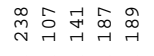 & & 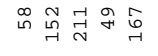 & 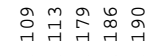 & $\stackrel{\sim}{\underset{\sim}{N} \underset{\sim}{\sim}}$ & $\begin{array}{ll}0 \\
m\end{array}$ \\
\hline 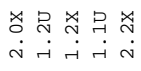 & 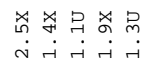 & 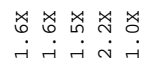 & 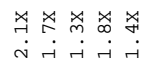 & & 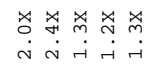 & 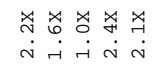 & & \\
\hline & & 皇 & & & & 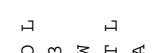 & 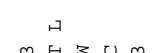 & \\
\hline$\vec{z}$ & 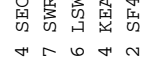 & 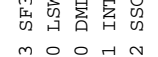 & 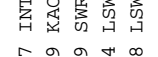 & 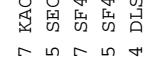 & 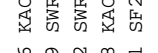 & 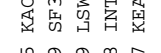 & 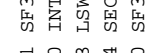 & \\
\hline ( & $\dot{n}$ & 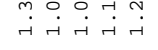 & 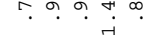 & & ri & & में & \\
\hline 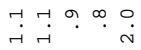 & 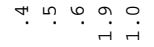 & 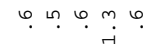 & 年 & . & ص. & . & صै. & 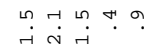 \\
\hline 웅 굼ㅇำ & 䎹 & 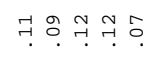 & 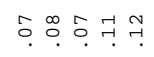 & 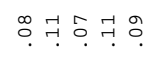 & 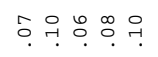 & 웅. 웅. & 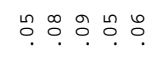 & 0 \\
\hline$\stackrel{\infty}{\sim} \stackrel{\circ}{\circ}$ & 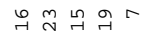 & 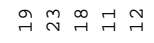 & 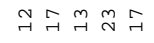 & 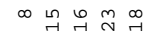 & $\stackrel{\sim}{N} \underset{\sim}{N} \vec{H}$ & 육두요 & $\stackrel{\sim}{\sim} \oplus \stackrel{\infty}{\infty}$ & 5 \\
\hline 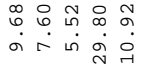 & 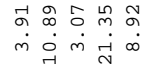 & 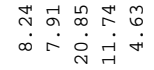 & 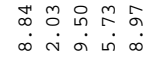 & 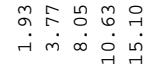 & 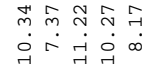 & & 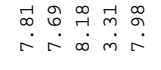 & \\
\hline & 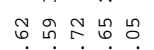 & 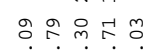 & 요유. & mpr & b. & 운 & & 8 \\
\hline & & $\dot{\circ} \dot{\sim} \dot{\sim} \dot{m} \dot{m} \dot{\sim}$ & $\stackrel{\oplus}{\rightarrow} \stackrel{\infty}{\sim} \stackrel{i}{N}$ & & & वे & & \\
\hline 员总 & 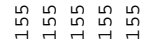 & 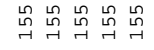 & 虽总号号虽虽 & 虽虽号虽虽虽出 & 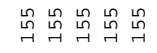 & 虽虽虽虽出虽出 & 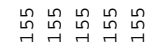 & \\
\hline & નુ & 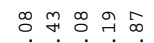 & 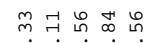 & बr. & $\stackrel{\infty}{\infty}$ & ִn. & 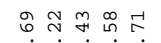 & $\sim$ \\
\hline & กิ่า & 이구 & N & & & & & \\
\hline & $\rightarrow$ & ने & $\rightarrow$ & ने & न & ने ने & $a$ & 80 \\
\hline & 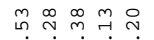 & & & & & & & \\
\hline & के & vi & & & & & & \\
\hline 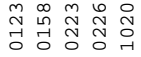 & 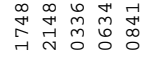 & 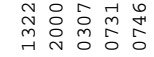 & 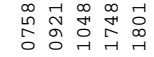 & & & 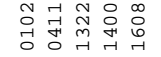 & 离节 & \\
\hline & 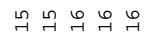 & 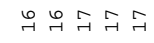 & 둑 & $\tilde{F}=$ & ने ने & $\stackrel{\circ}{\sim}$ & 00 & $\vec{N} \vec{N}$ \\
\hline & 월 열 & 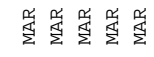 & 蒌迸 & 爱獣 & 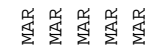 & 造迸 & $\frac{\pi}{2}$ & \\
\hline & 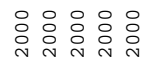 & : & : & : : & & & & \\
\hline
\end{tabular}




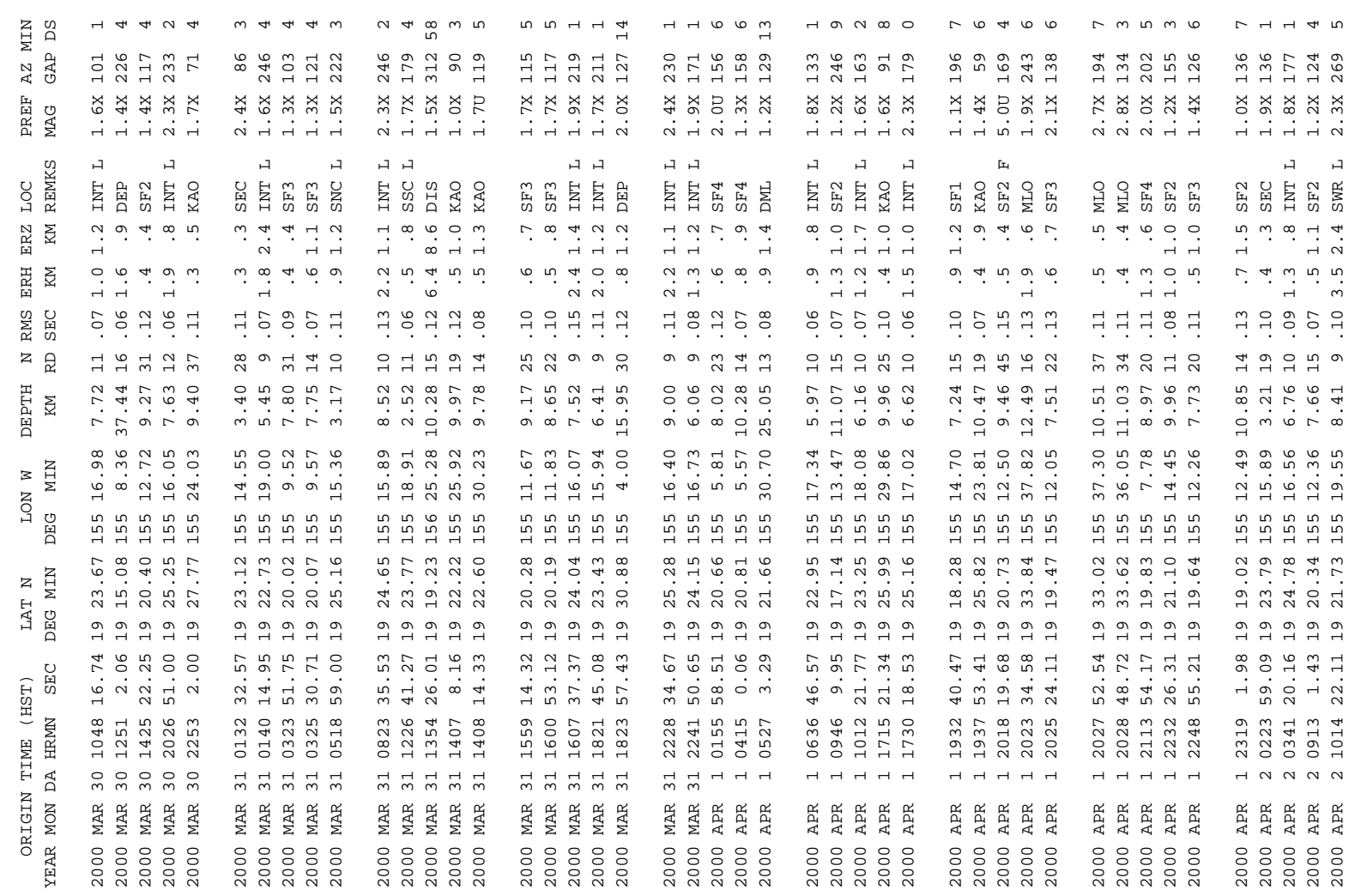

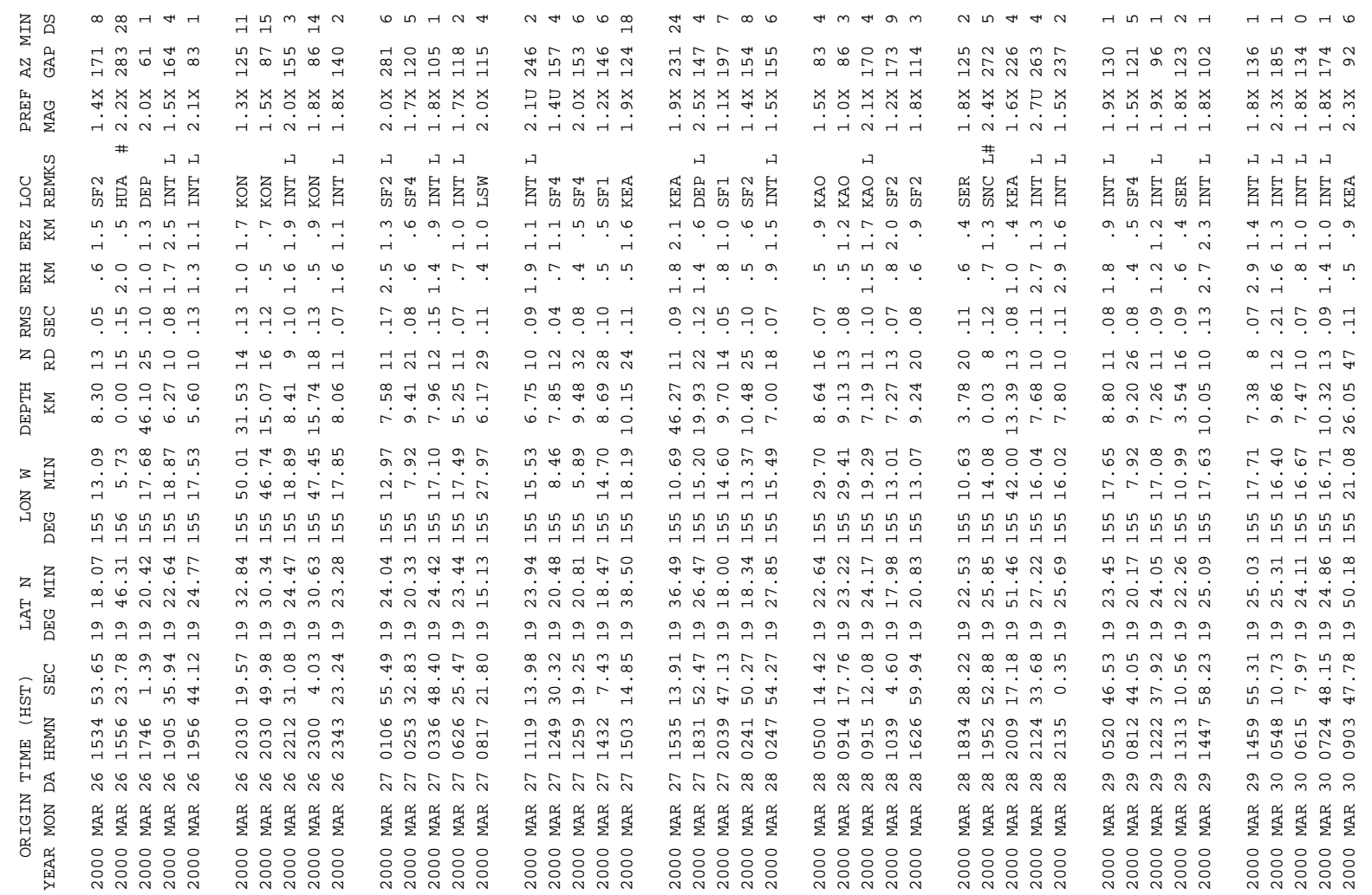




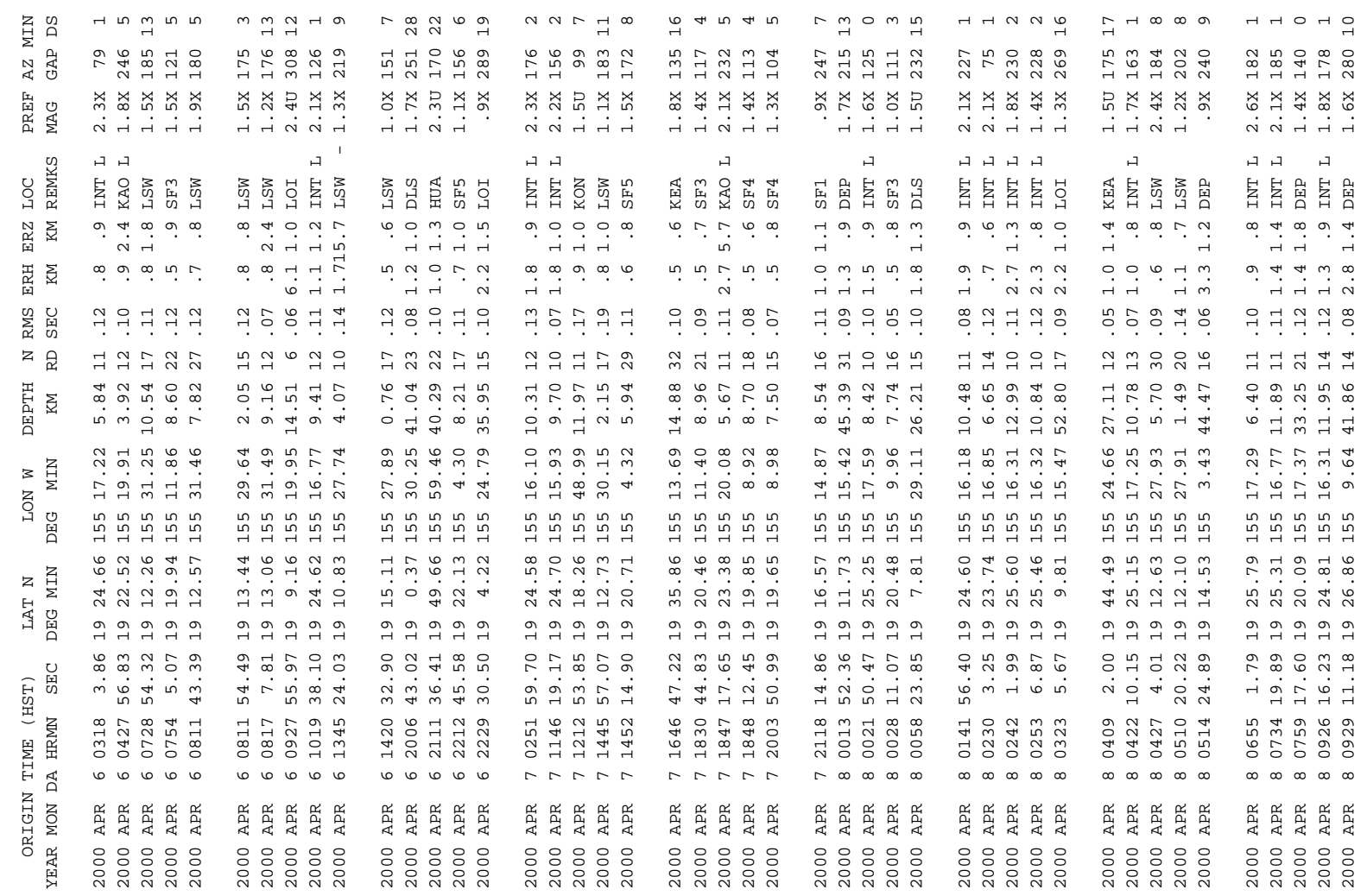

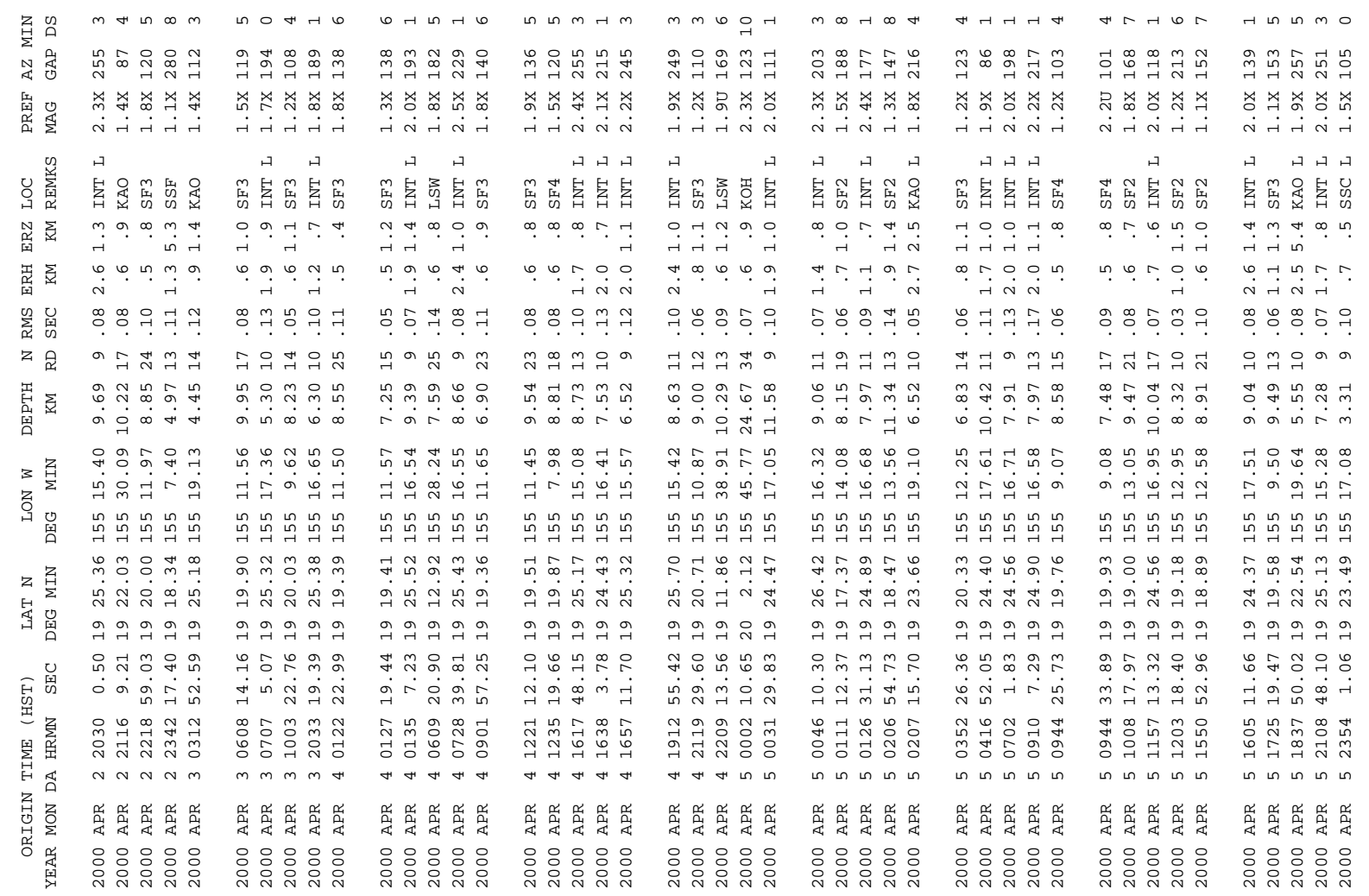




\begin{tabular}{|c|c|c|c|c|c|c|c|c|}
\hline ம & $\vec{F}$ & $\exists ન \dashv \backsim \stackrel{\infty}{N}$ & 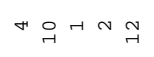 & $6 \pi r$ & ص & $\approx \approx a$ & $\infty$ & $m$ \\
\hline & & 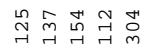 & 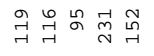 & ${ }_{\substack{M \\
\hdashline}}^{3}$ & & & & \\
\hline & 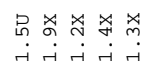 & 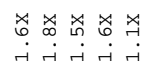 & 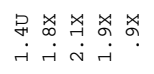 & 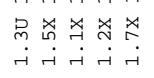 & 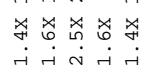 & 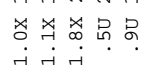 & & \\
\hline के & & 倪 & 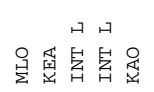 & 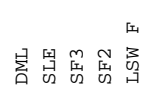 & & & 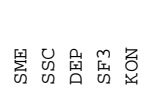 & \\
\hline & & & ( & & & & $r$ & \\
\hline & & $\stackrel{6}{\cdot .}$ & 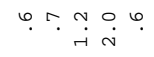 & & 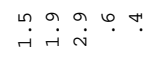 & & $\dot{r}$ & s \\
\hline & 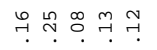 & $\stackrel{\infty}{\infty}$ & 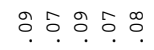 & ㅇํㅇ & ㅇ․․ & & & \\
\hline & $\pi m$ & $\infty \sim 7$ & స & 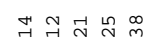 & $\vec{F} \vec{\exists}$ & & & \\
\hline & & $\infty \infty$ & & & 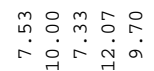 & & & \\
\hline & & & & & & & & \\
\hline & & & & & & & & \\
\hline & 怘虽 & 品 & 号号 & 虽出号 & 虽虽 吕 & 另 & 吕号 & 吕 \\
\hline & & & & & & & & \\
\hline & & & & & & & & \\
\hline & & & & & & & & \\
\hline & & & & & & & & \\
\hline & & & & & & & & \\
\hline & & & & & & & & \\
\hline & & & & & & & & \\
\hline & : : & : : & 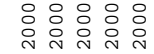 & & 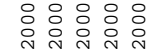 & & & \\
\hline
\end{tabular}

\begin{tabular}{|c|c|c|c|c|c|c|c|c|}
\hline 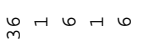 & $H N G N O$ & $H \neg \underset{N}{ } \rightarrow N$ & $n \pi N \sim N$ & Nmarm & ๓ & $60+76$ & $\infty \backsim \vDash 00$ & $6 \pi r \rho^{\circ} \sigma$ \\
\hline 芦总 & 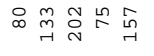 & 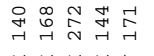 & 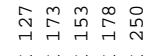 & 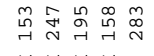 & 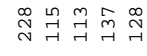 & 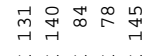 & 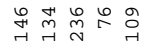 & 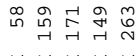 \\
\hline 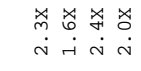 & 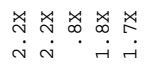 & 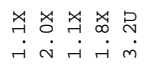 & 굴 & 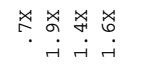 & 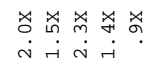 & 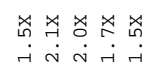 & 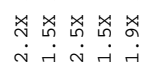 & 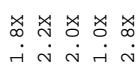 \\
\hline 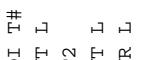 & 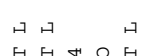 & 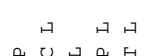 & & & $\vec{A}$ & Н & & 山山 \\
\hline 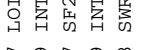 & 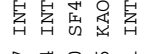 & 畕总茾畐葍 & 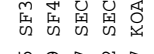 & & 鬼总 & 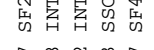 & 证量 & \\
\hline 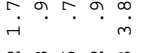 & ᄀ. & 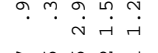 & ?. & لُ & $\stackrel{m}{m} \underset{i}{n} \stackrel{9}{i}$. & 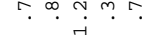 & 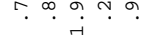 & $\stackrel{n}{i}$ \\
\hline 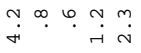 & 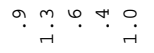 & †. & 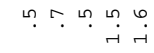 & 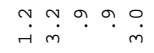 & 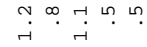 & ب. & ". & \\
\hline 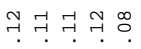 & 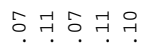 & 울 움구 국 & . & $\stackrel{\sim}{\sim} \stackrel{\sim}{\sim}$ & 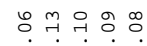 & 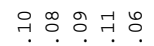 & 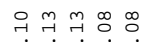 & 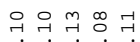 \\
\hline 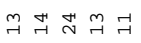 & 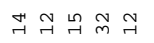 & 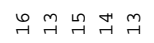 & 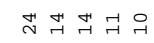 & 웍고 & 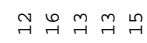 & $\stackrel{\sim}{\sim} \vec{\sim} \sim \vec{\sim}) \stackrel{\sim}{\sim}$ & $\underset{\sim}{N} \underset{\sim}{\sim} \underset{\sim}{\sim} \stackrel{\sim}{\sim} \vec{్}$ & $\stackrel{\sim}{m} \stackrel{\infty}{\sim} \stackrel{\infty}{\sim}$ \\
\hline 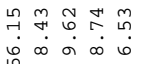 & 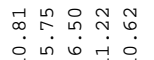 & 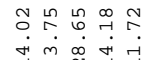 & 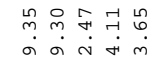 & 5 ำ స & ऽ。 & 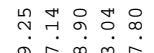 & न & 89 \\
\hline ond & 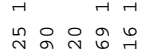 & $\begin{array}{l}0 \\
0\end{array}$ & แn & 윰 出 & 17 & 85 & & $m^{\infty} \infty$ \\
\hline 我 & $\dot{\square}=$ & & & & $\dot{\sigma} \vec{\sigma} \dot{\sigma} \dot{\sigma}$ & 寈 & $\because$ & $\stackrel{\dot{\sim}}{\sim} \stackrel{\dot{\sim}}{\sim}$ \\
\hline 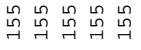 & 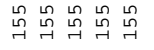 & 虽虽点虽出虽 & 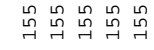 & 㤩虽 & 怘虽虽虽出 & 总虽虽虽虽虽 & 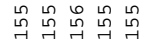 & 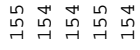 \\
\hline 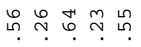 & 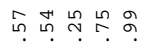 & m. & 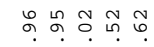 & $\stackrel{N}{\circ}$ & ले ต़. & ڤึ. & ? & 다. \\
\hline 陨资 & 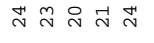 & $\stackrel{\pi}{N} \stackrel{\sim}{\sim} \stackrel{0}{\sim}$ & 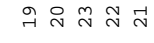 & $m$ & $\stackrel{\infty}{\sim} \underset{\gamma}{-1}$ & $\Rightarrow$ & $\stackrel{m}{\sim}$ & $\stackrel{6}{\infty} \stackrel{2}{\sim}$ \\
\hline$\stackrel{\infty}{\rightarrow}=$ & 국웅 & 국우 & 국 & 00 & 국 & 국의 & & ने \\
\hline & ğ & & & & 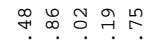 & छु. & & $\infty_{\infty}^{\infty} 5$ \\
\hline & & & & & $\dot{m} \dot{\sim} \dot{\alpha} \dot{\sigma}$ & 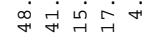 & & $\because m-1$ \\
\hline 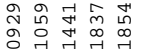 & 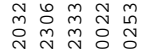 & +0 & $m$ & 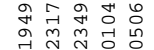 & 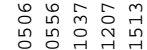 & 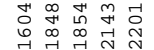 & & 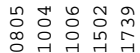 \\
\hline & & & & & & & & \\
\hline & 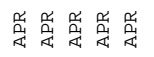 & 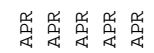 & 岁 & 完家 & 崖崖宸 & 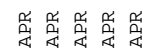 & 岩岩 & 乐我 \\
\hline & 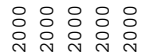 & & & & : & 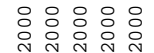 & & 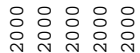 \\
\hline
\end{tabular}




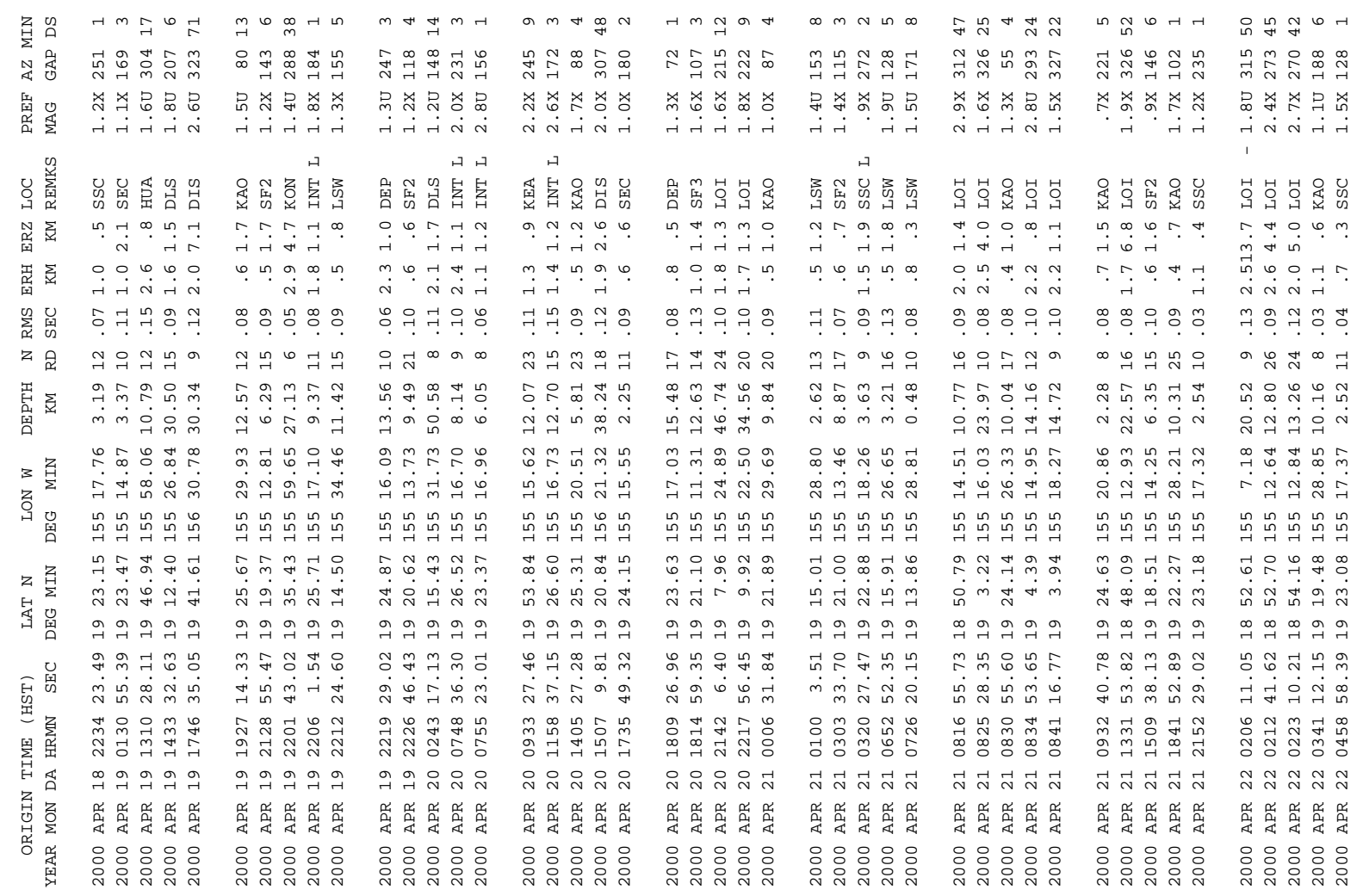

\begin{tabular}{|c|c|c|c|c|c|c|c|c|}
\hline$m \backsim \nabla N \pi$ & $\infty \backsim \infty \quad n \pi$ & $\curvearrowleft \Gamma+\curvearrowleft \curvearrowleft$ & $m+m \prod^{m} b$ & $\curvearrowleft \rightarrow r \sim \underset{\sigma}{m}$ & $a \sigma m \underset{\pi}{\infty}+$ & 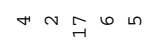 & 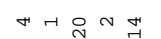 & ๓の オ \\
\hline 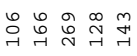 & 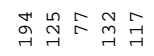 & $\overrightarrow{\vec{g}} \underset{\sim}{\vec{d}} \stackrel{\infty}{\infty} \underset{N}{\vec{V}} \vec{G}$ & 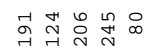 & 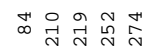 & 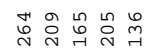 & 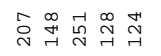 & 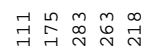 & 苟哭 \\
\hline 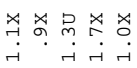 & 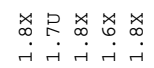 & 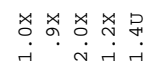 & 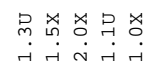 & 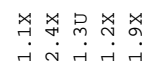 & 品 盾 & 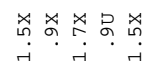 & 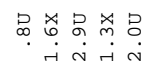 & $x$ \\
\hline & & H & & Н & & & & \\
\hline$\pi$ & 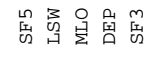 & 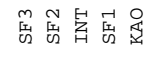 & 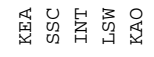 & 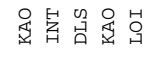 & 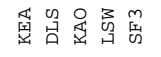 & 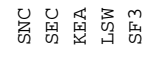 & 曾崮吕总置 & 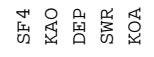 \\
\hline & ? & مُ & 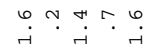 & 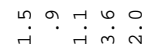 & 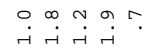 & 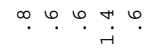 & 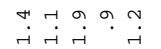 & ㄱ. \\
\hline & & ร. ร.9. & تِ & 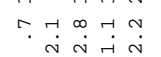 & ז艹 & ?ִ. & 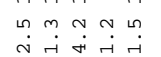 & \\
\hline 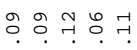 & 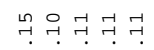 & 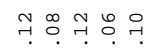 & 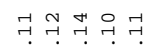 & 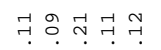 & ๆ ธุ̣. & 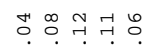 & 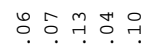 & 우융 \\
\hline & $\stackrel{\sim}{\sim} \underset{\sim}{\sim}$ & $\stackrel{\infty}{\sim} \underset{\sim}{\sim} \underset{\sim}{\sim} \underset{\sim}{\sim} \stackrel{\infty}{\sim} \mathcal{\sim}$ & 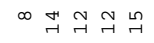 & 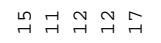 & 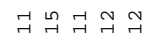 & $\exists$ 워 & 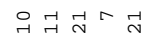 & 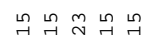 \\
\hline & 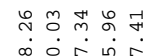 & 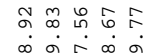 & 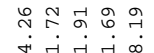 & 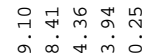 & $\stackrel{0}{N}$ 둥 & $r$ & $\infty$ & $a$ \\
\hline & & $\infty$ & 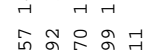 & 105 & $\infty$ & $0 \pi 60$ & in 0 & \\
\hline & $\circ \stackrel{\dot{0}}{\mathrm{~d}} \mathrm{q}$ & 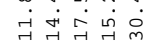 & $\dot{\sim} \dot{\sim} \dot{\sim} \stackrel{\infty}{\sim} \dot{m}$ & 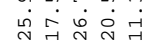 & 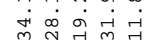 & $\underset{\sim}{+} \underset{\sim}{\vec{\sim}} \stackrel{\infty}{\infty} \stackrel{\dot{d}}{N}$ & 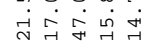 & 6 \\
\hline & 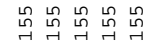 & 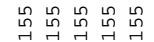 & 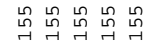 & 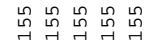 & 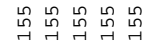 & 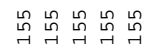 & 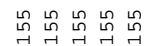 & 虽嵒 \\
\hline & & Non & 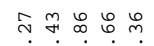 & เุำㅇำ ำ? & & & m. & \\
\hline & $\dot{\mathrm{N}}$ & $\dot{\sigma} \dot{二} \dot{\circ}$ & $\ddot{n}$ & $\stackrel{\infty}{\sim} \stackrel{\dot{\sim}}{\sim}:$ & & & & \\
\hline & $\rightarrow$ 가 & ने & 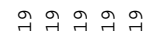 & ने ने ने ने & $\sigma \sigma$ & $a$ & $a \sigma$ & ने ने ने \\
\hline & ڤ̊. & $\vec{b}$ & & 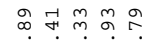 & & & & \\
\hline & & $\stackrel{\infty}{\sim} \stackrel{\infty}{N}$ & 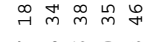 & नं ले & -4 & & & \\
\hline & 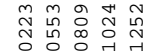 & 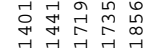 & 总 & 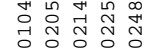 & $\begin{array}{ll}0 & 0 \\
0 & m \\
0 & + \\
0\end{array}$ & $N$ & $\stackrel{\sim}{N}$ & 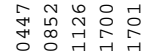 \\
\hline & & $\underset{\sim}{\stackrel{0}{*}}$ & & 두두 & 곡드 & & $r न$ & $F$ \\
\hline & & 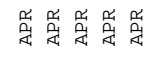 & 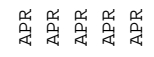 & 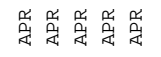 & 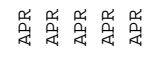 & 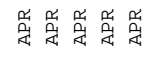 & 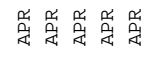 & 岁焉 \\
\hline & & & $N \sim N N$ & 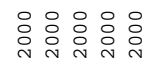 & & & & \\
\hline
\end{tabular}




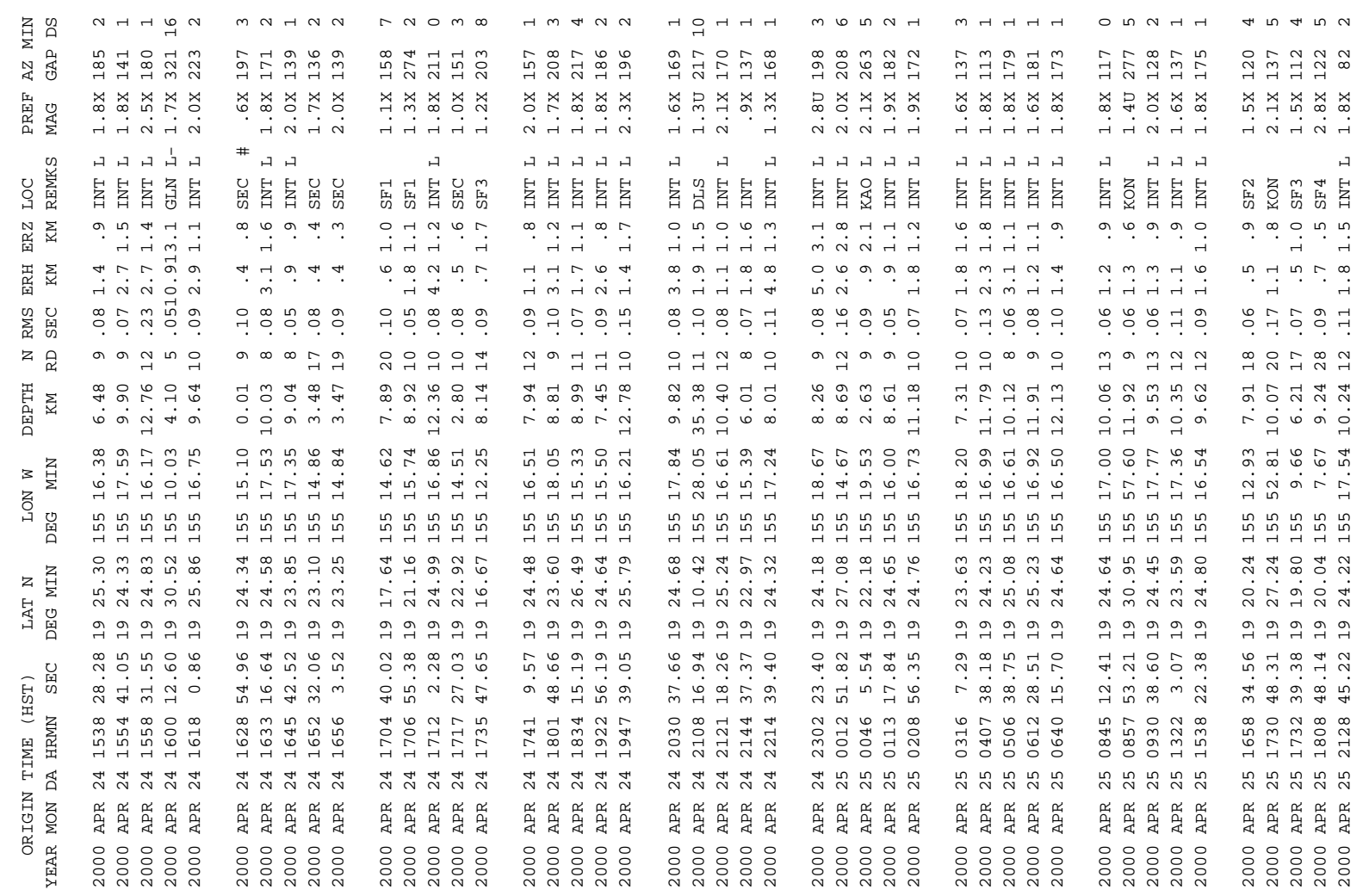

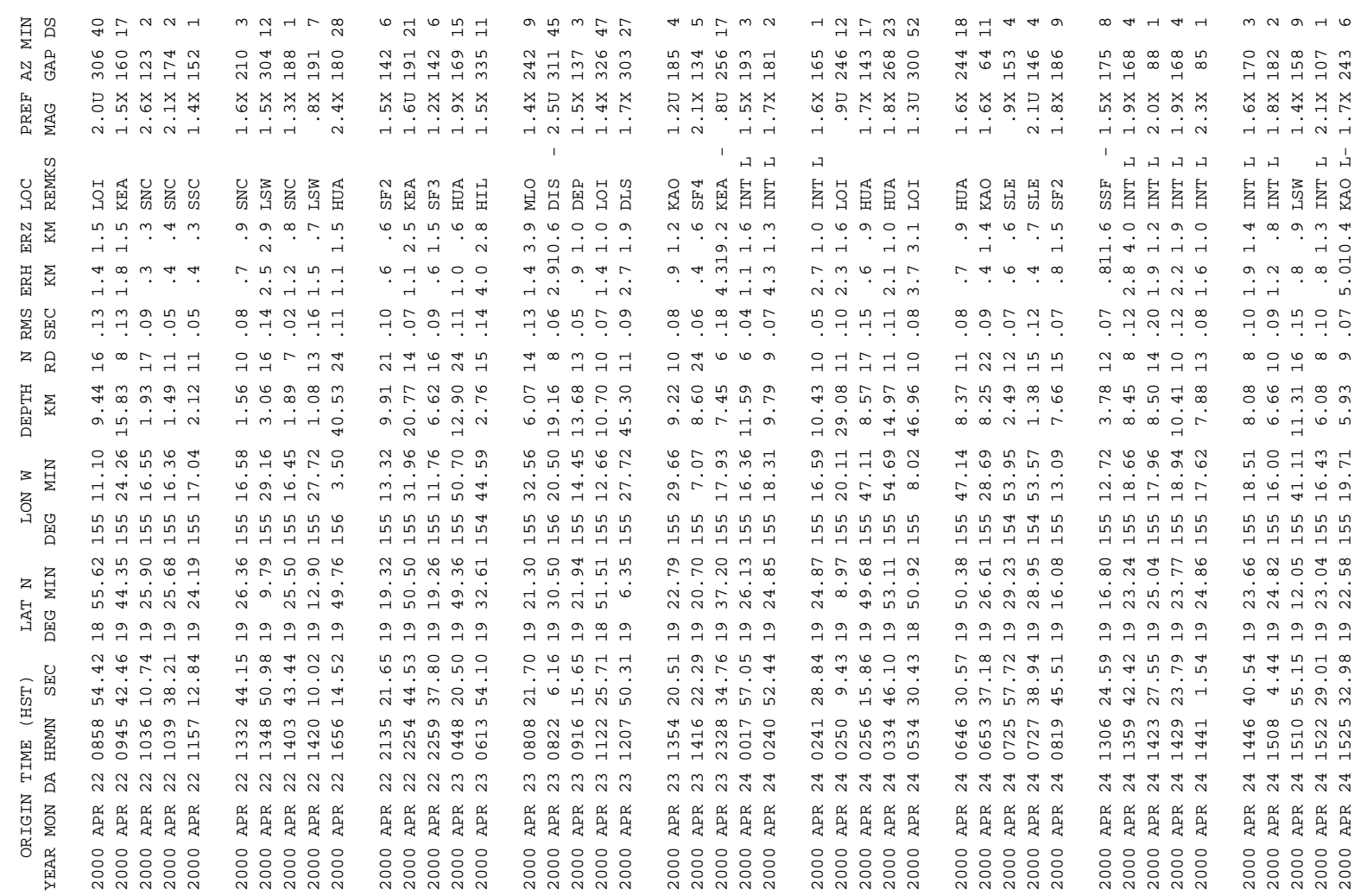




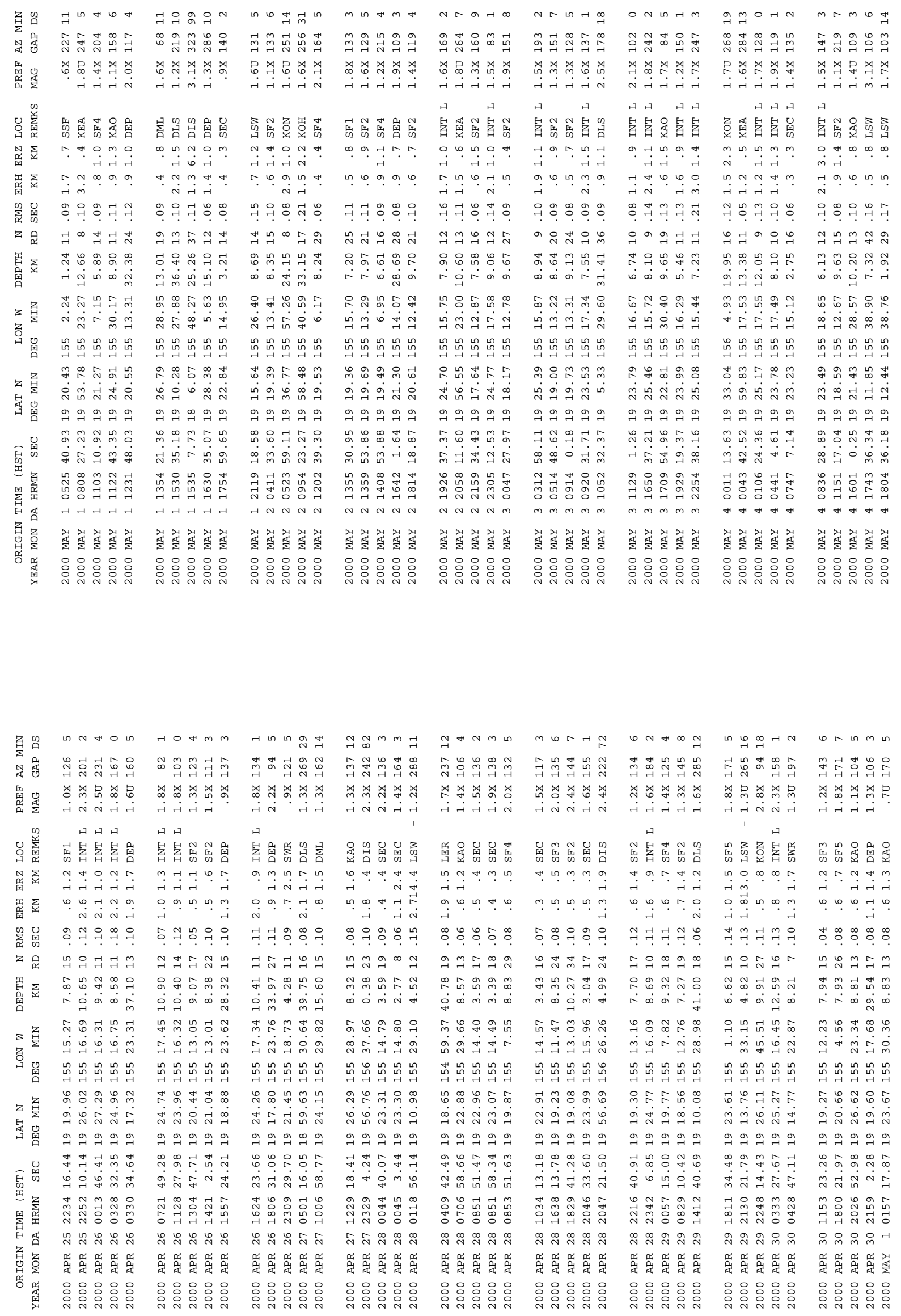




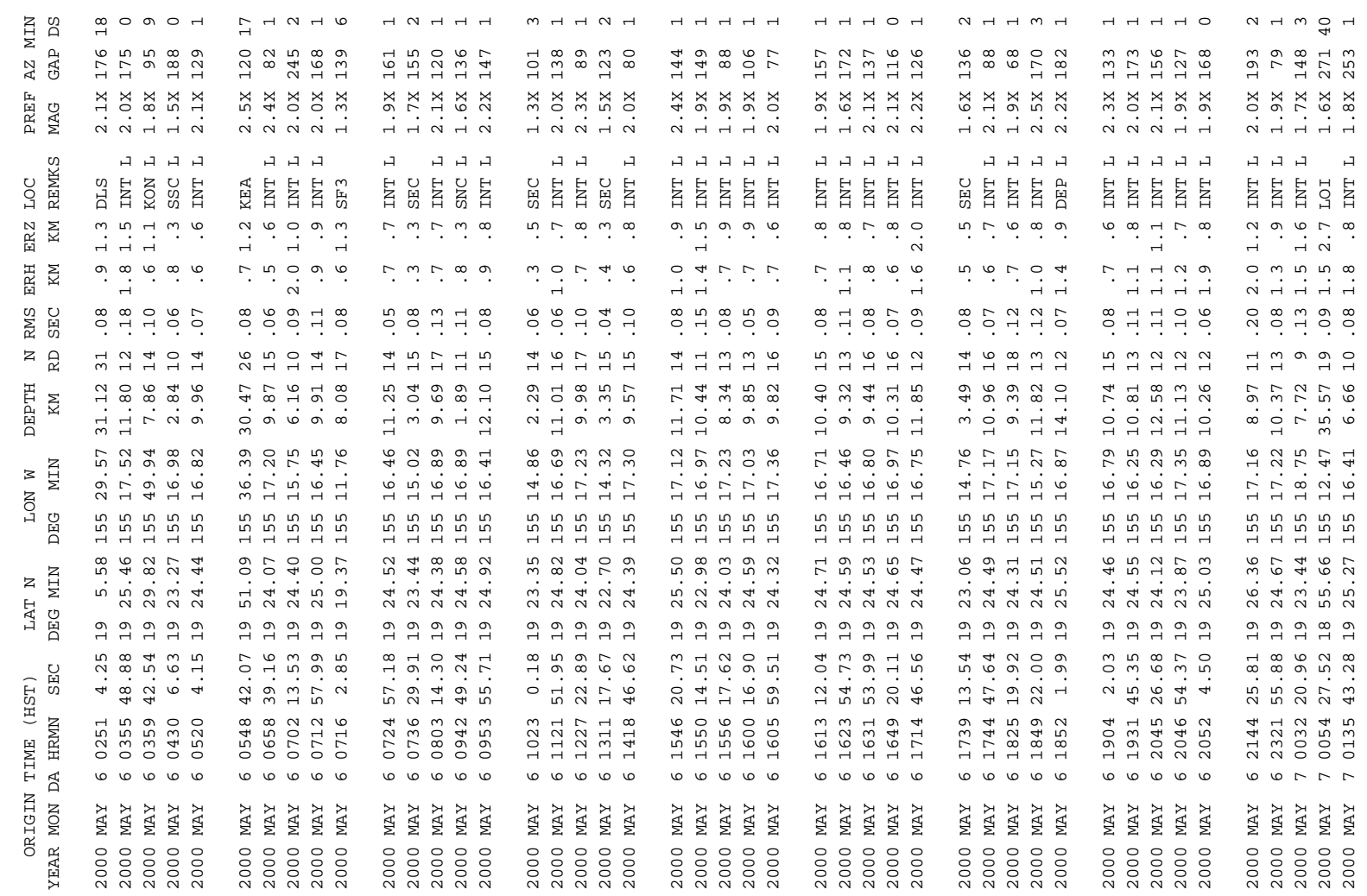

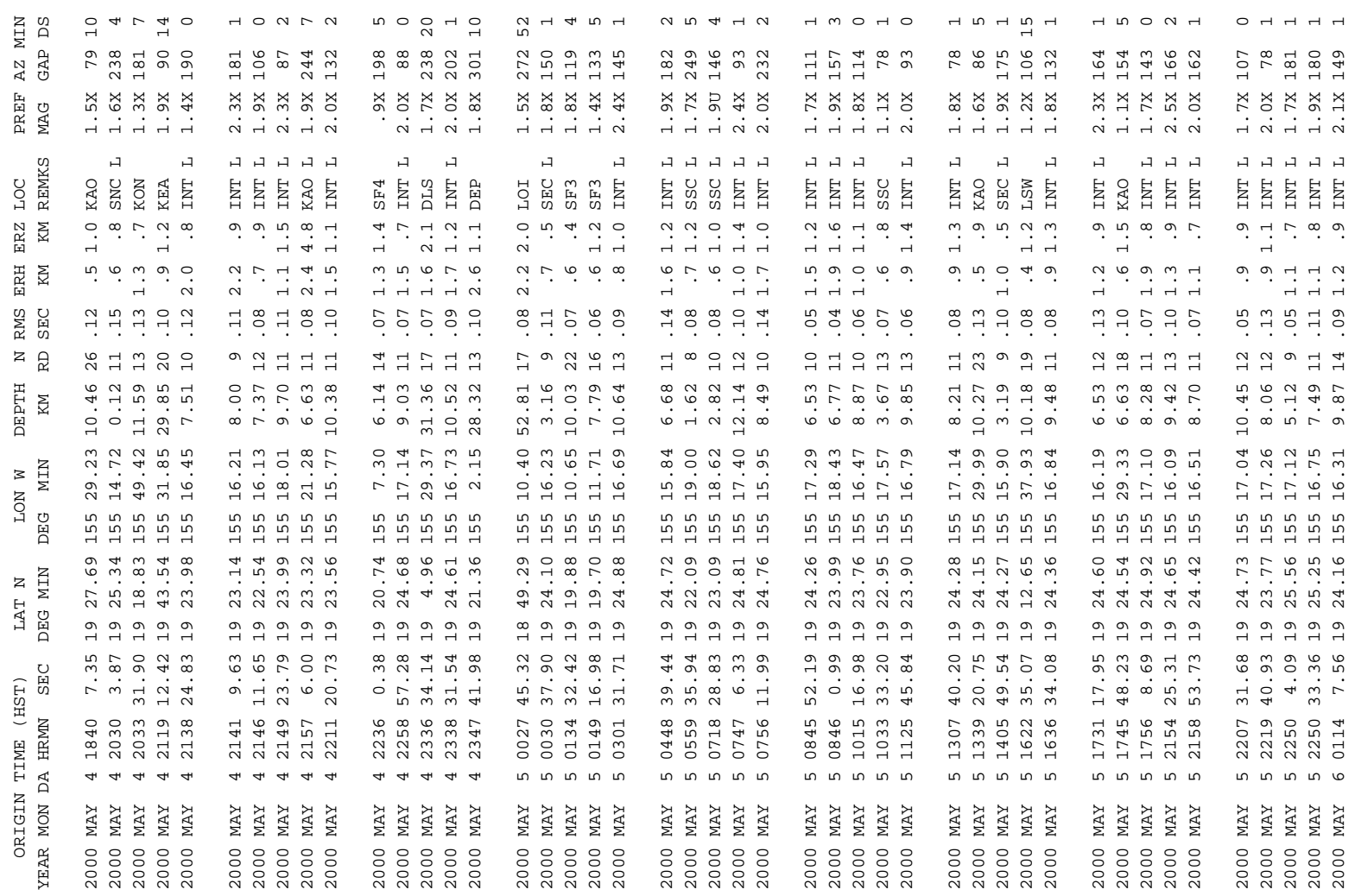




\begin{tabular}{|c|c|c|c|c|c|c|c|c|}
\hline$H \sim$ ก & $\neg \sim m \underset{\sim}{\infty}$ & $0404 \mathrm{n}$ & Om $m+n$ & $4 \rightarrow 04 t$ & מ & ○m & $h \rightarrow O N H$ & tra \\
\hline 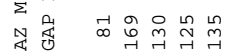 & 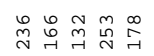 & 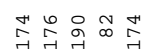 & 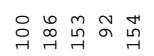 & 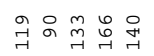 & 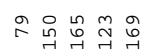 & 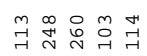 & 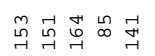 & 닥 \\
\hline$\stackrel{\substack{x \\
j}}{i}$ & 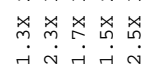 & 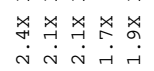 & 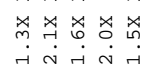 & 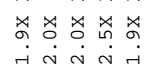 & 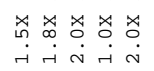 & 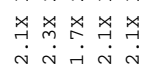 & 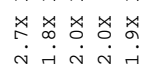 & N. \\
\hline & & 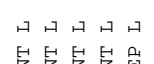 & & Инан & & & حн & \\
\hline & & & & & & & & \\
\hline 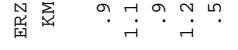 & 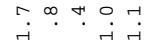 & 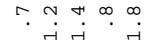 & م. & 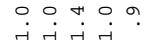 & 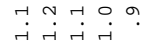 & . & o. & . \\
\hline 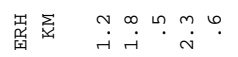 & 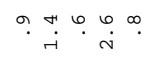 & تُ & : & o. & : & $\underset{\pi}{\pi} \cdot$ & 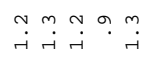 & 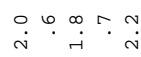 \\
\hline 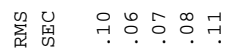 & 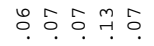 & 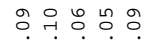 & 임 & 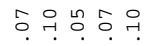 & $\stackrel{5}{\circ} \stackrel{\infty}{\circ}$ & ? & o. & \\
\hline 윽 & 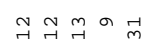 & 욱ㄱ & $a$ & $\exists \exists$ & $\exists$ & N & $m \approx$ & ब \\
\hline & & & & & & & & \\
\hline & & & & & & & & \\
\hline & & & & & & & & \\
\hline & & & & & & & & \\
\hline & & & & & & & & \\
\hline & 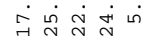 & & & & & & & \\
\hline & $\Rightarrow \rightarrow$ & & & & & & 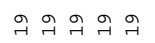 & \\
\hline & & & & & & & & \\
\hline & 的势 & in & & & & & $\dot{4}$ & \\
\hline & & 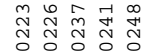 & & & & & & \\
\hline & & & & & & & & \\
\hline & $\frac{x_{1}^{2}}{2}$ & है & & & & & & \\
\hline & & & & & & & & \\
\hline
\end{tabular}

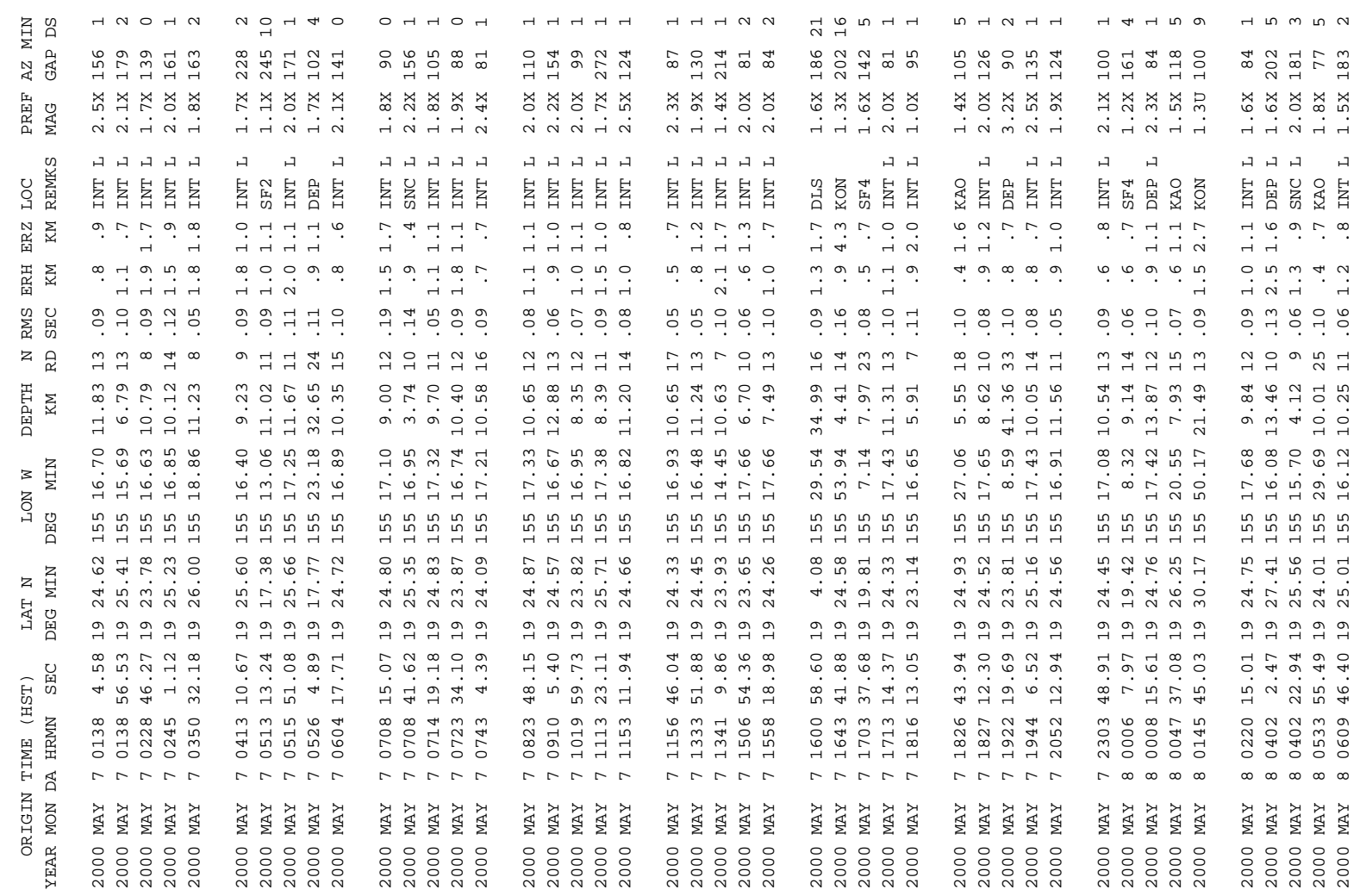




\begin{tabular}{|c|c|c|c|c|c|c|c|c|}
\hline$N \rightarrow \curvearrowleft m-H$ & ththt & $H \rightarrow N O H$ & $-1 \backsim \sim \underset{N}{\infty}$ & ת 6 N & ๓ & - $\backsim \backsim 6 r$ & $m \underset{m}{m}+\underset{N}{\stackrel{m}{H}}$ & 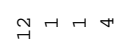 \\
\hline$\vec{g} \underset{F}{F}$ & 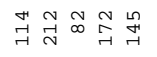 & 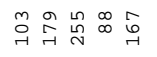 & $\stackrel{\bullet}{ت} \vec{G}$ & 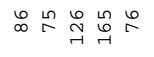 & 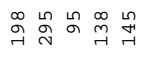 & 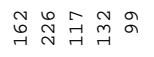 & 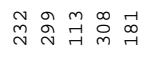 & 곳 $\sigma^{\circ} \underset{\sim}{\sim}$ \\
\hline 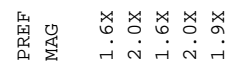 & 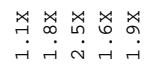 & 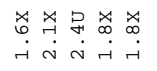 & 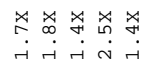 & 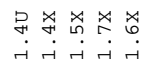 & 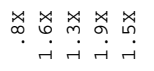 & 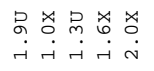 & 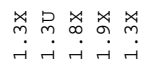 & 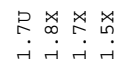 \\
\hline 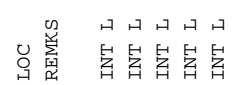 & 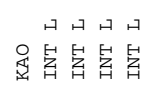 & 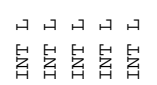 & 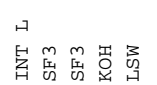 & 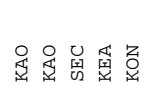 & 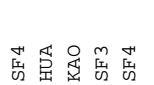 & 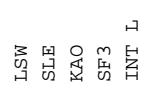 & 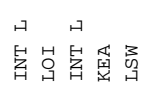 & 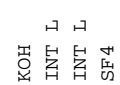 \\
\hline ْ: & 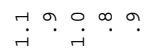 & ${ }_{i}^{m} \rightarrow{ }_{i}^{-1}$. & & & & $\stackrel{\varphi}{\cdot} \underset{+}{+}$ & $\vec{r} \stackrel{\vec{m}}{\ddot{m}} \stackrel{0}{\dot{r}}$ & 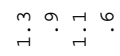 \\
\hline 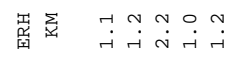 & 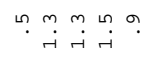 & $\begin{array}{l}0 \\
\dot{H} \\
\dot{H}\end{array}$ & 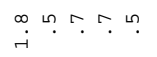 & 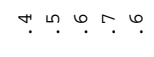 & 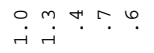 & 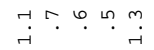 & 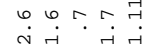 & 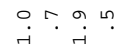 \\
\hline$\stackrel{m}{?} ?$ & 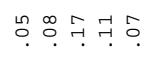 & ำ ํำ & 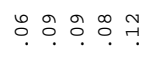 & 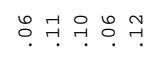 & 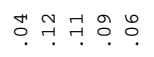 & 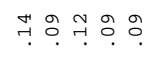 & 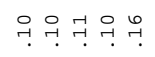 & 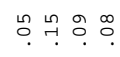 \\
\hline 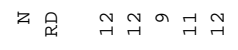 & 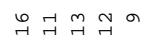 & a욱욱ㄱㄱ & 유굑 & 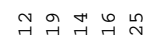 & $\underset{\sim}{\sim} \tilde{A} \vec{N} \underset{N}{N}$ & 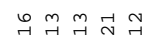 & 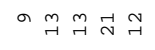 & $\underset{\sim}{\sim} \stackrel{m}{\sim} \underset{\sim}{N}$ \\
\hline 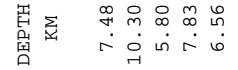 & 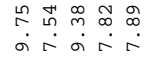 & 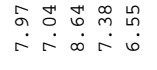 & 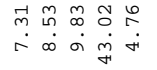 & 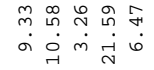 & 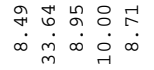 & 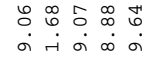 & 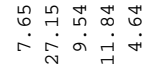 & \\
\hline 象 & $\frac{\pi}{5}$ & 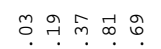 & & No & & 足 & 象 & $\because 7$ \\
\hline & $\dot{\sim}=$ & 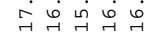 & $\dot{\sim} \dot{ }$ & $\dot{m}$ is & $\dot{r}$ & $\dot{4}$ & & \\
\hline 虽出总总㽞 & 虽虽虽虽虽虽 & 虽虽虽虽虽虽 & 虽虽虽虽虽虽 & 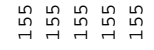 & 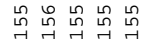 & 虽莒虽号虽虽 & 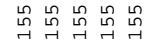 & 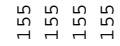 \\
\hline เै. & 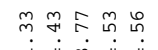 & 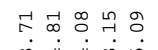 & 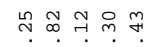 & 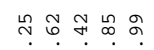 & $\infty$ & ने भे. & o. & נִ \\
\hline & $\vec{N}$ & & $\underset{\sim}{\pi}$ & & & & & \\
\hline & $\stackrel{\theta}{r}$ & 욱요 & के & مे & 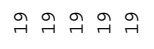 & Fे & St & 구 ने ने \\
\hline 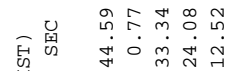 & 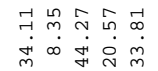 & 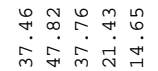 & 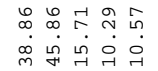 & 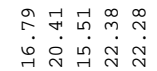 & 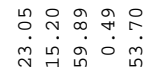 & 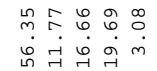 & & 7 \\
\hline $\begin{array}{ll}0 & 0 \\
0 \\
0\end{array}$ & 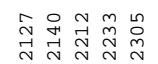 & 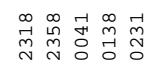 & 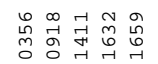 & 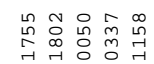 & m & v & & \\
\hline$\rightarrow$ & 윽윽으윽음 & 욱욱ㄱ & & $\vec{F} \cdot$ & & & & $\stackrel{n}{\rightarrow}$ \\
\hline$\Sigma \Sigma \Sigma$ & 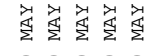 & 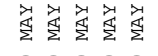 & & 离离 & & & & \\
\hline 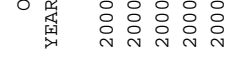 & 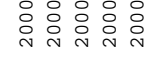 & 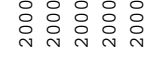 & 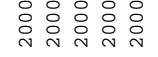 & $\begin{array}{ll}0 \\
0 \\
0\end{array}$ & $\begin{array}{ll} \\
\text { na }\end{array}$ & 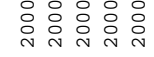 & & \\
\hline
\end{tabular}

\begin{tabular}{|c|c|c|c|c|c|c|c|c|}
\hline 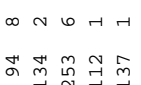 & 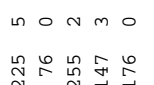 & 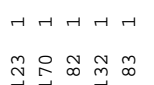 & 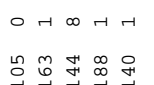 & 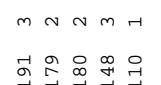 & 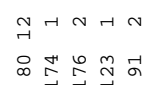 & 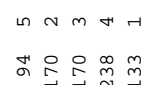 & 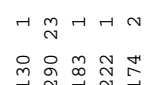 & 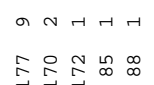 \\
\hline $\begin{array}{ll}\hat{r} \\
\end{array}$ & 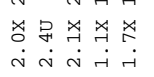 & 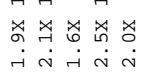 & 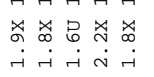 & 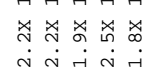 & 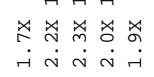 & 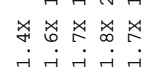 & 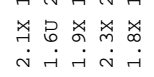 & $\vec{H} \dot{\sim} \vec{H} \dot{H}$ \\
\hline 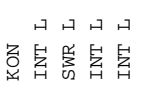 & 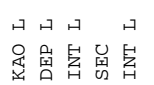 & 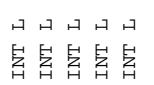 & 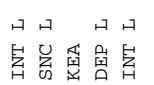 & 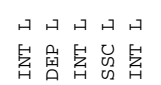 & 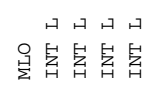 & 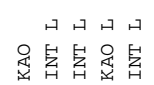 & 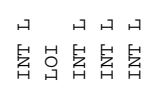 & 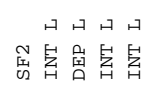 \\
\hline 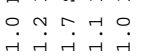 & 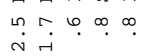 & $\stackrel{\infty}{\sim} \underset{i}{\sim} \dot{\sim}_{\dot{r}}^{\infty}$. & 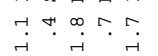 & 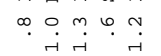 & : & 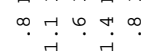 & : & ت. \\
\hline ๑.9. & 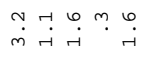 & 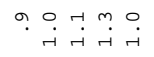 & 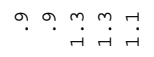 & 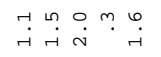 & 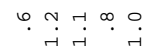 & 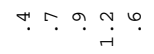 & 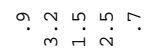 & ب. \\
\hline 구ำ ㅇํำ & 웅 नำำ & 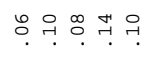 & $\stackrel{0}{\circ}:$ & 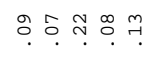 & 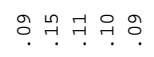 & 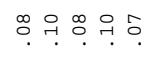 & \& & $\because \circ$ \\
\hline 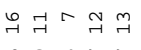 & 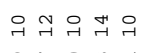 & $\exists ન \exists ન ્$ & 둥ㅇㅇㅇ & $\sigma \simeq \underset{\exists}{ } \simeq \exists$ & 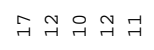 & 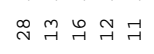 & 국움 & 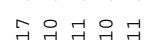 \\
\hline 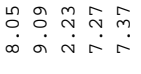 & 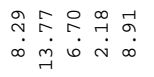 & 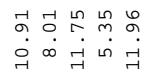 & 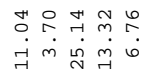 & $\begin{array}{l}m \\
0 \\
0\end{array}$ & 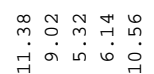 & 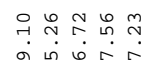 & 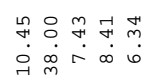 & $\begin{array}{lll}7 & 0 \\
7 & 0 \\
\infty & 0 & 0\end{array}$ \\
\hline 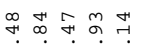 & 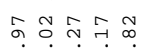 & 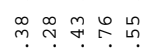 & 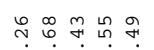 & 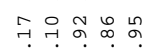 & & 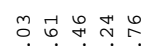 & $y-1$ & 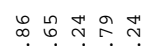 \\
\hline in & 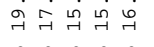 & 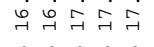 & 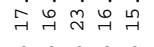 & 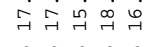 & 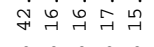 & $\dot{t}$ & if & \\
\hline 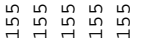 & 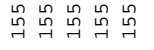 & 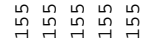 & 怘虽总虽虽 & 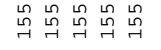 & 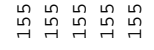 & 虽虽虽虽虽虽 & 虽总总 & 虽总虽 \\
\hline गे. & 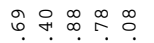 & 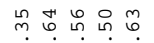 & & 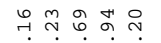 & in & ?. & & \\
\hline$\stackrel{\infty}{\sim} \stackrel{\infty}{\sim}$ & $\stackrel{n}{\sim} \stackrel{n}{\sim}$ & 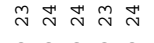 & & $\approx$ & & . & & \\
\hline 90 & 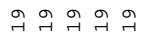 & 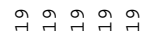 & & 0 & $\Leftrightarrow \sigma g$ & $\theta \sigma 0$ & 욱 의 욱 & $\because$ \\
\hline & ㅇำ & ถึ? & & $\infty$ & & m. & & \\
\hline & $m \neq 0$ & m $\tilde{m}$ & & F & & & & $\sqrt{37} \sqrt{2}$ \\
\hline & $\vec{N} \vec{N} \vec{N} N$ & 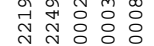 & & 00 & & & & \\
\hline & & & & & & & & \\
\hline & 출 철 & 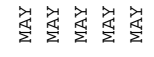 & 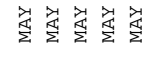 & $\frac{\pi}{2} \frac{S_{2}}{2}$ & & 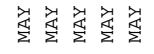 & & \\
\hline & & & & & & & & $\pi N$ \\
\hline
\end{tabular}




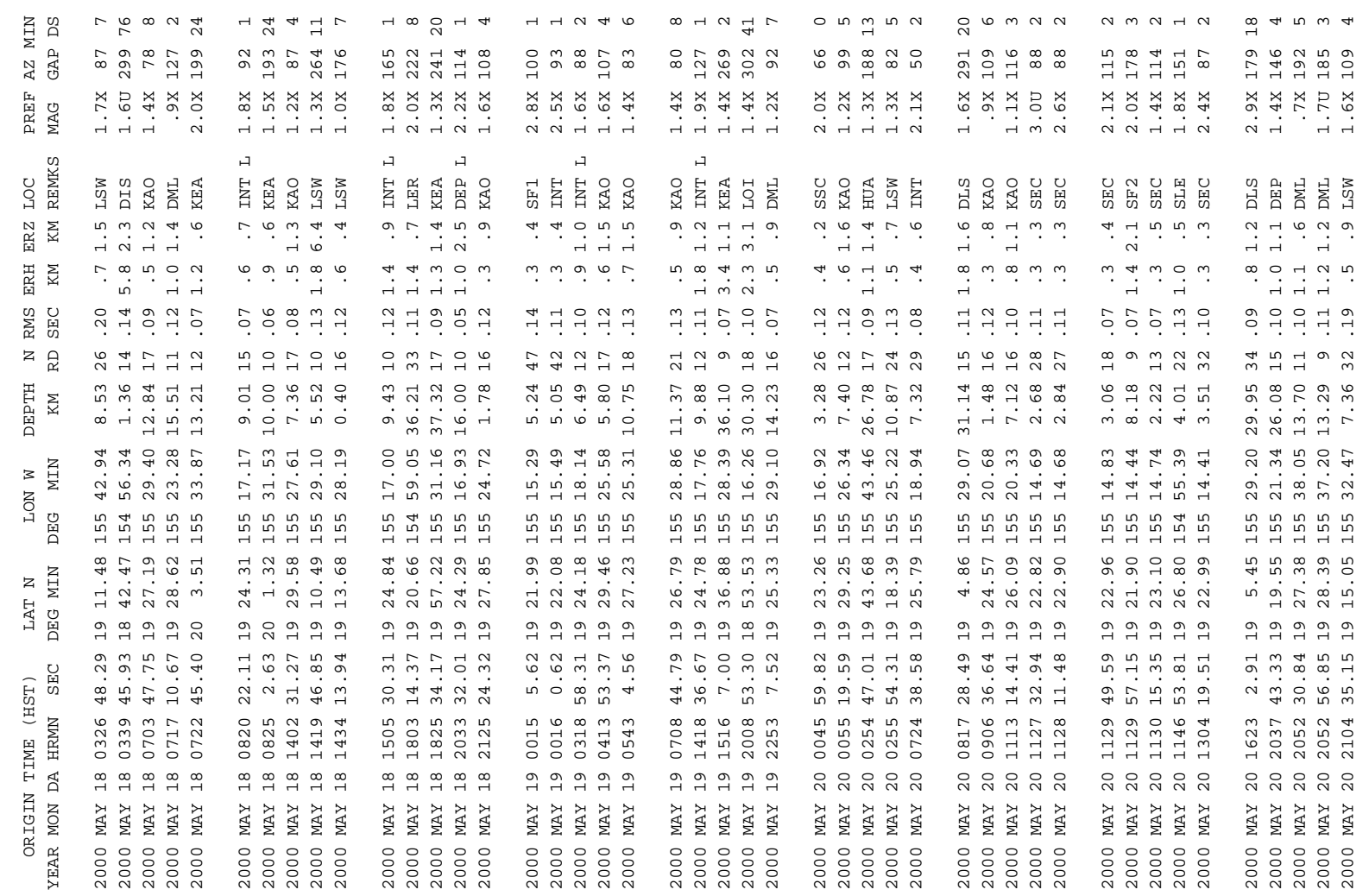

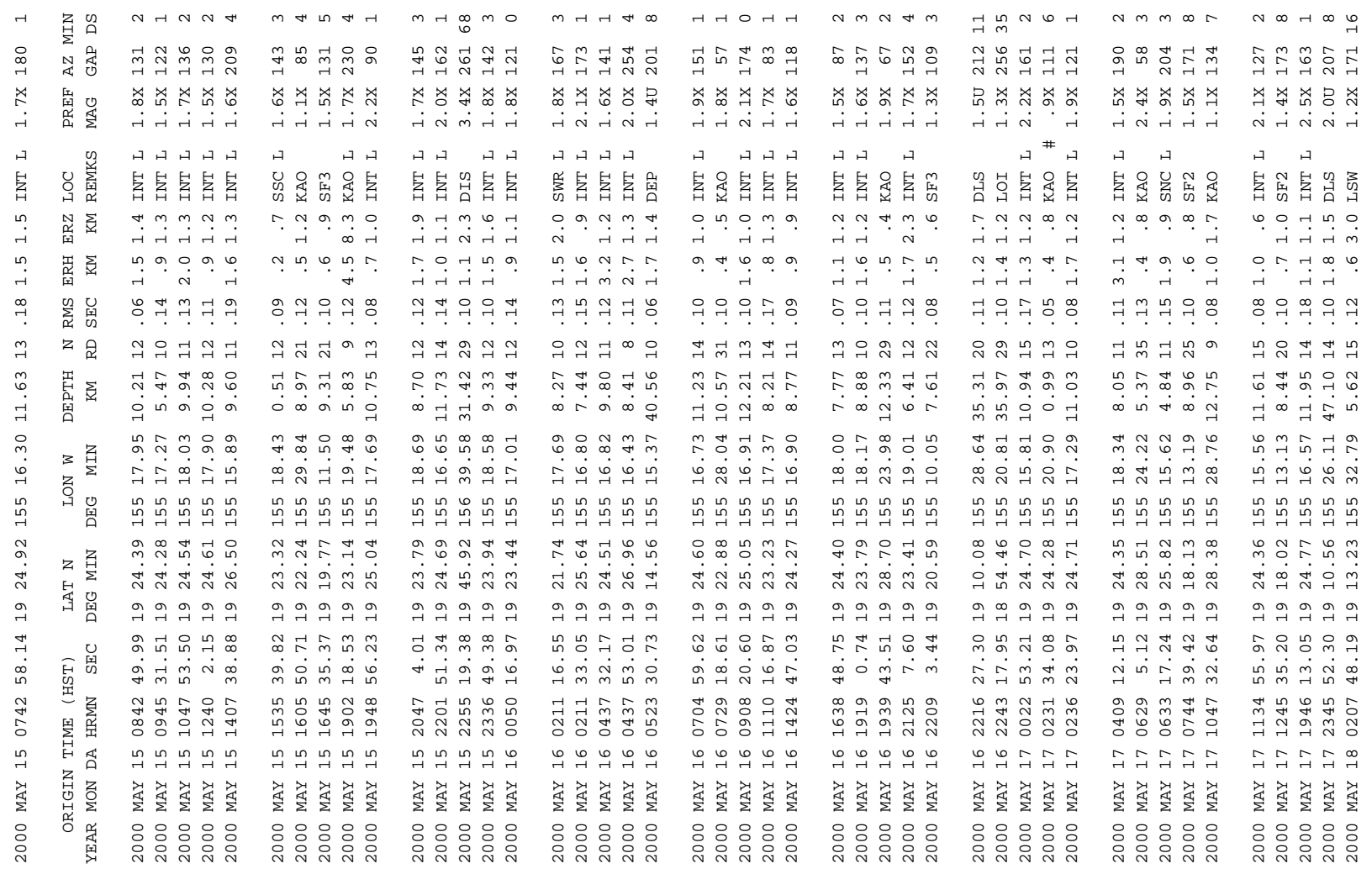




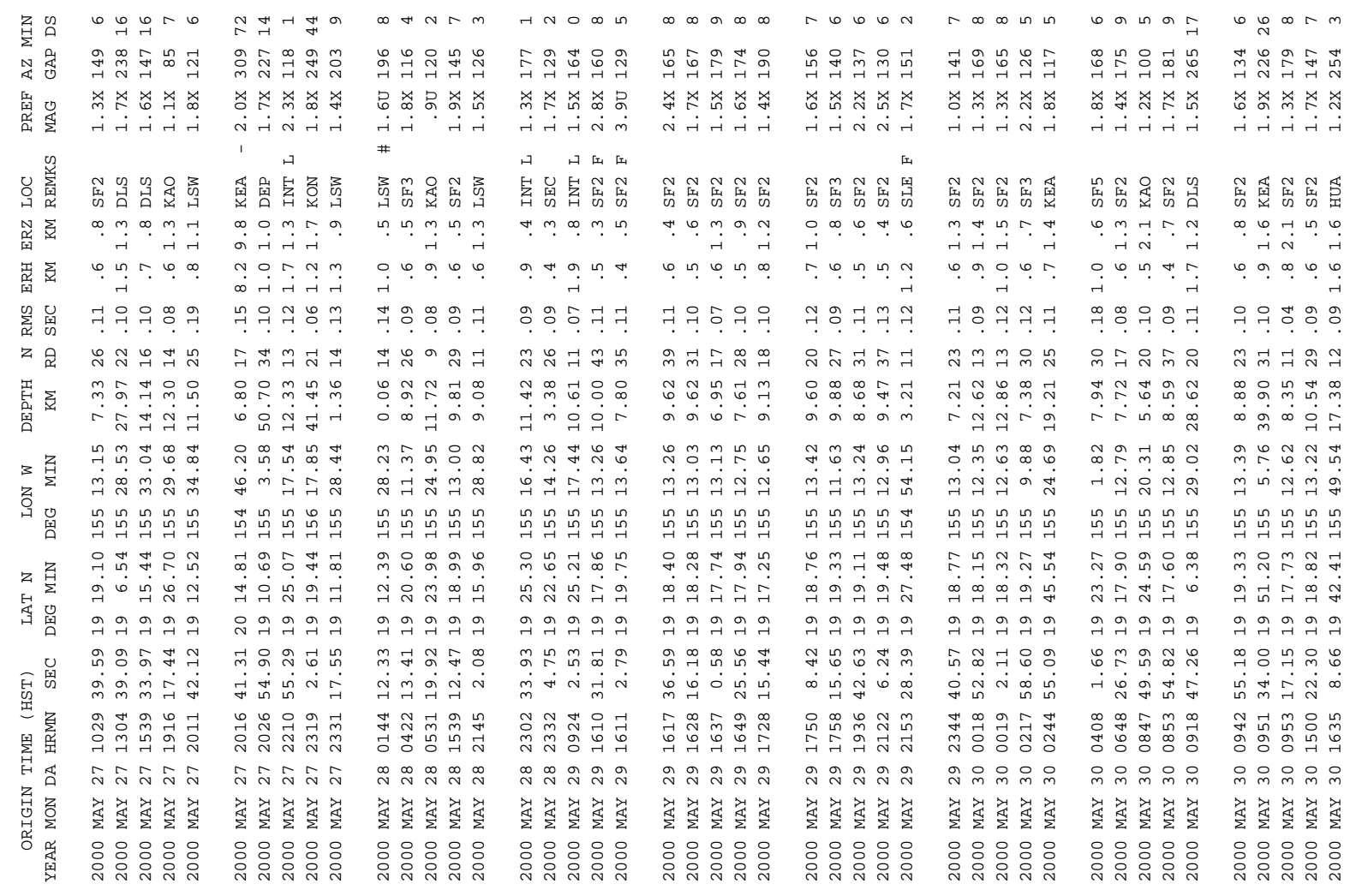

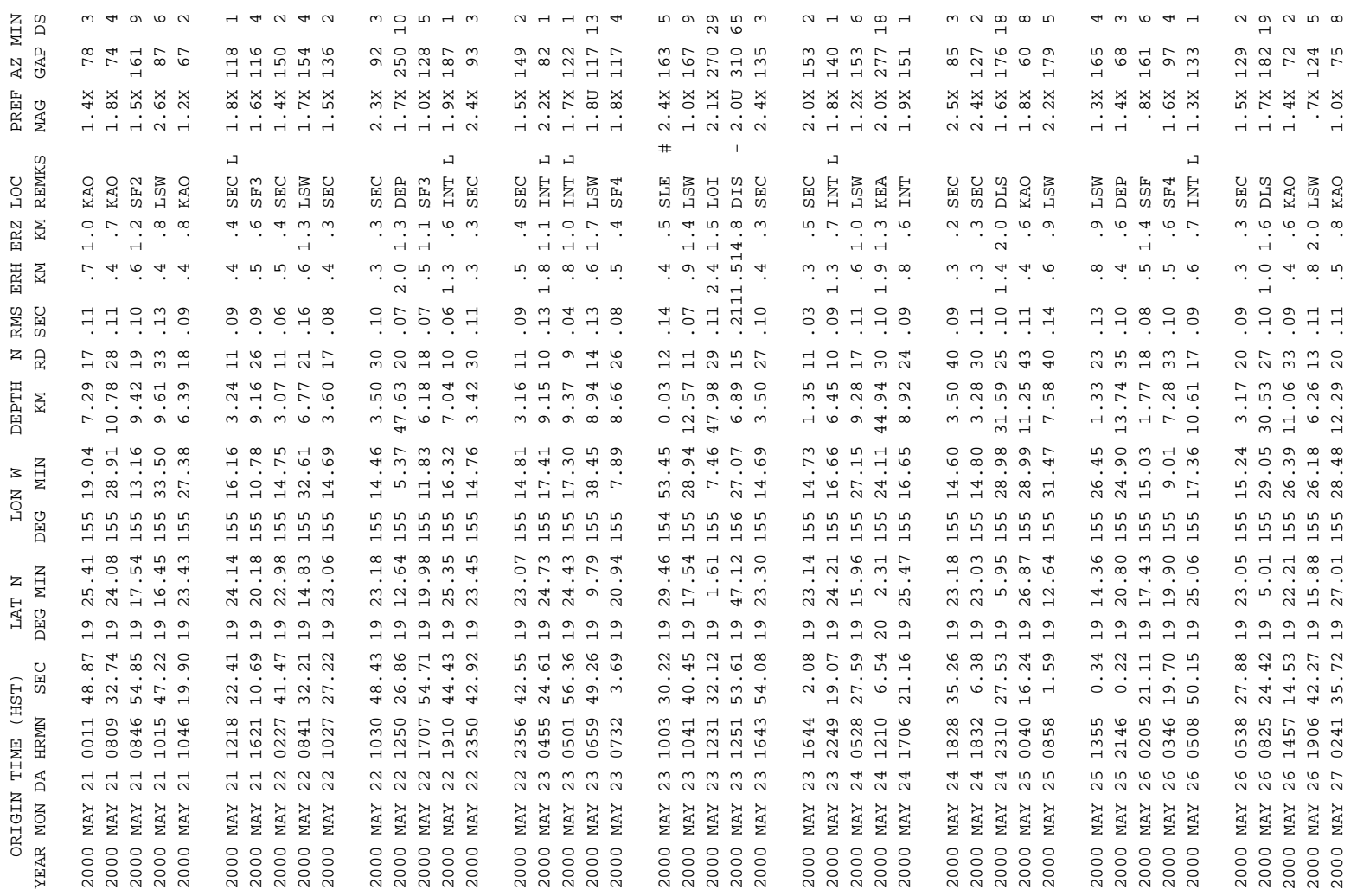




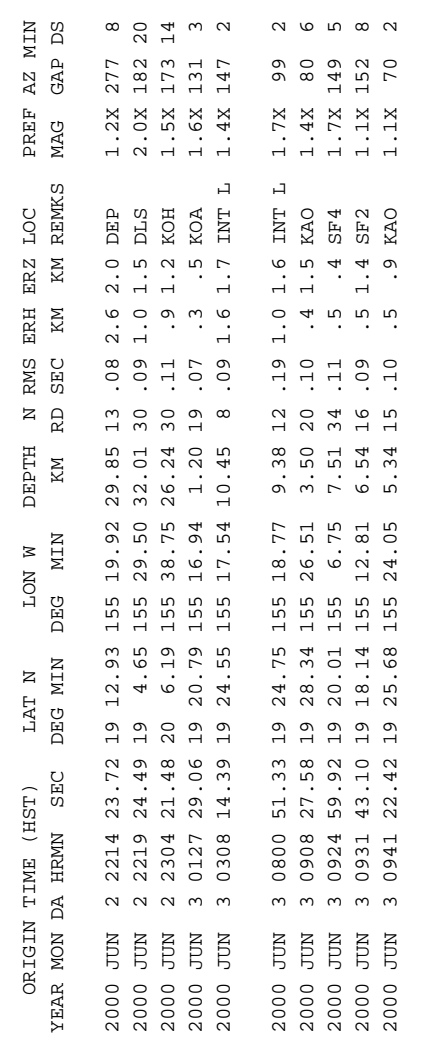

๓ ㅇㅓㄸ 凹

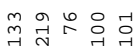

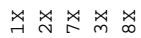

मं में

超留总若

m. 웅.

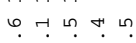

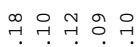

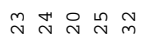

우에

क

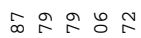

வें

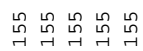

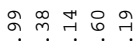

म्न

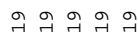

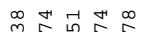

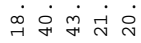

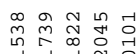

लm nो

总总总房

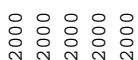

$\infty \infty \infty \sim \infty$

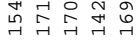

瓜 $\underset{m}{x} \underset{-}{x} \underset{\sim}{x}$

نं में

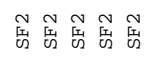

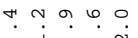

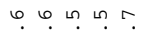

구 거굳ㅇㅇㅇㅇㅇㅇ.

궁ำ

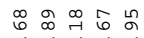

कें

익규

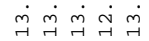

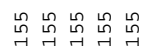

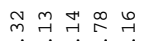

$\stackrel{\infty}{\infty} \stackrel{\infty}{\infty} \stackrel{\infty}{\infty} \stackrel{\infty}{\infty} \stackrel{\infty}{\sim}$

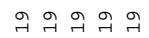

गีำ ธิ

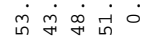

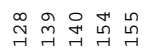

小㥀

总总总总总

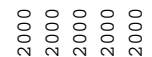

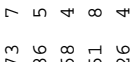

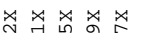

तं नं

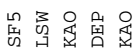

m 00.90

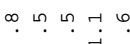

끅옹의궉의

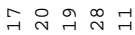

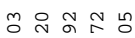

히일

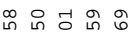

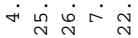

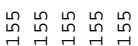

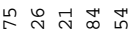

웅ㅎㅇ

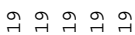

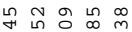

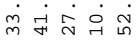

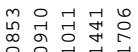

小标

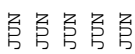

: : $: 0:$

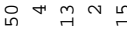

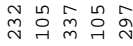

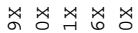

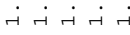

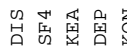

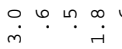

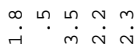

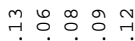

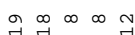

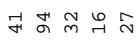

के

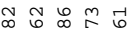

लं $\dot{m}$ ने

员员员员虽

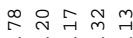

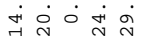

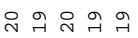

망요 유

बें户 min

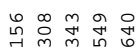

낸

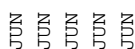

융ㅇㅇㅇㅇ
6ே几๘凸

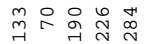

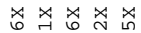

में

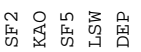

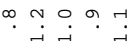

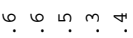

ㄱ. ㄱ․ ㄱำ 국

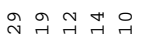

幽

क नें

각

mं山्

虽虽虽虽虽

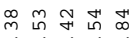

ने $\stackrel{\infty}{\infty} \underset{\sim}{\infty} \dot{\sim}$

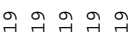

乩岀牙兵㚙

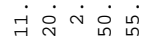

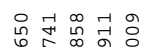

๒ ᄂ 以

总总总总

응웅ㅇㅇㅇㅇㅇ
걱 $\infty$ 엉

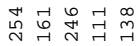

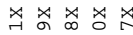

में

总总总着最

‥ m mo

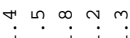

구국극ㄱㄱㅇㅡ

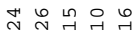

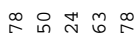

ने $\sigma \stackrel{\dot{~}}{\infty} \stackrel{\infty}{\sim}$

독ำ

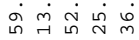

员员员员员

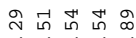

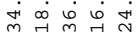

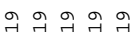

공 난다잉

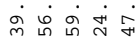

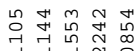

๒๒ら๒

总总总总总

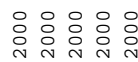

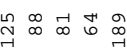

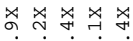

मंने में

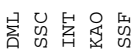

ㅜㅇ‥

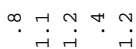

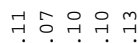

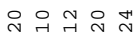

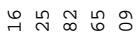

तें山ा के

운

$\dot{m} \underset{\sim}{\infty} \underset{\sim}{\infty} \stackrel{\infty}{\sim} \dot{\sim}$

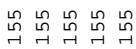

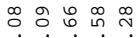

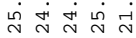

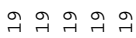

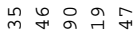

i

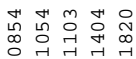

66666

总总房总总

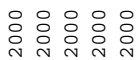

刍

$$
\text { 炎 }
$$$$
\text { 留 }
$$

岱

品

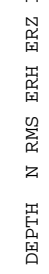

0006 -

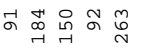
جে

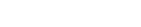

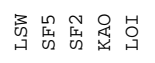
ㄴ. ㄴ. 6.9. ำ. ํ. ำ

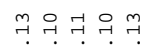
늠 สํำ

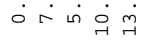
주의 궁

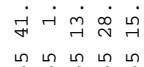

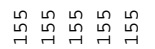

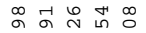
تं

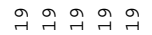

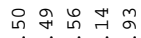
ले

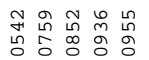
लेलेल $\vec{m} \vec{m}$

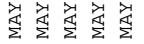

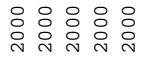

nhtn

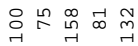

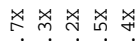
Н

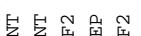

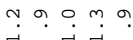
क. … .6 오욱우우금 그규워

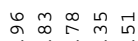
नेंबें

员

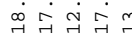
员员员员虽 艝 웅 வ்

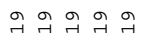

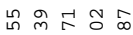
ㄴำ

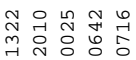
लेलनान

这娄台台䏍 :ㅇ: 융 a n 60 in

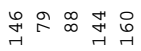

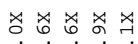
यें

म

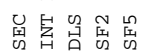
m. 구 굴. ㄱ. ต. ". ๓. ? ?

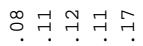

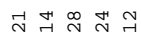

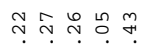
लं

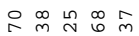
में 虽虽员员另虽 은용용요

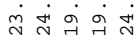

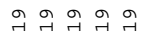

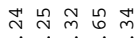
अ

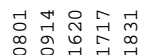
HनH न

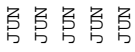
융유 m $r$ a d

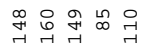

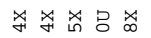
नंनंत्र

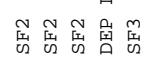
욱. ‥ ? ‥ 6.0 ㄴ.?

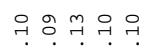
육하

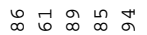
$\dot{0} \dot{\infty} \infty \underset{\vec{r}}{\dot{r}} \dot{r}$ ๑ 증ำ

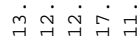
员员出员虽 ल ढ़ 車 ने न्न न्न ने ने 우 $\stackrel{\infty}{\sim} \stackrel{\infty}{\sim} \stackrel{\sim}{\sim}$ 吕

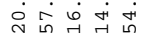

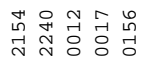
4
$4 \pi N N$

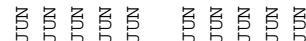

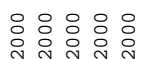




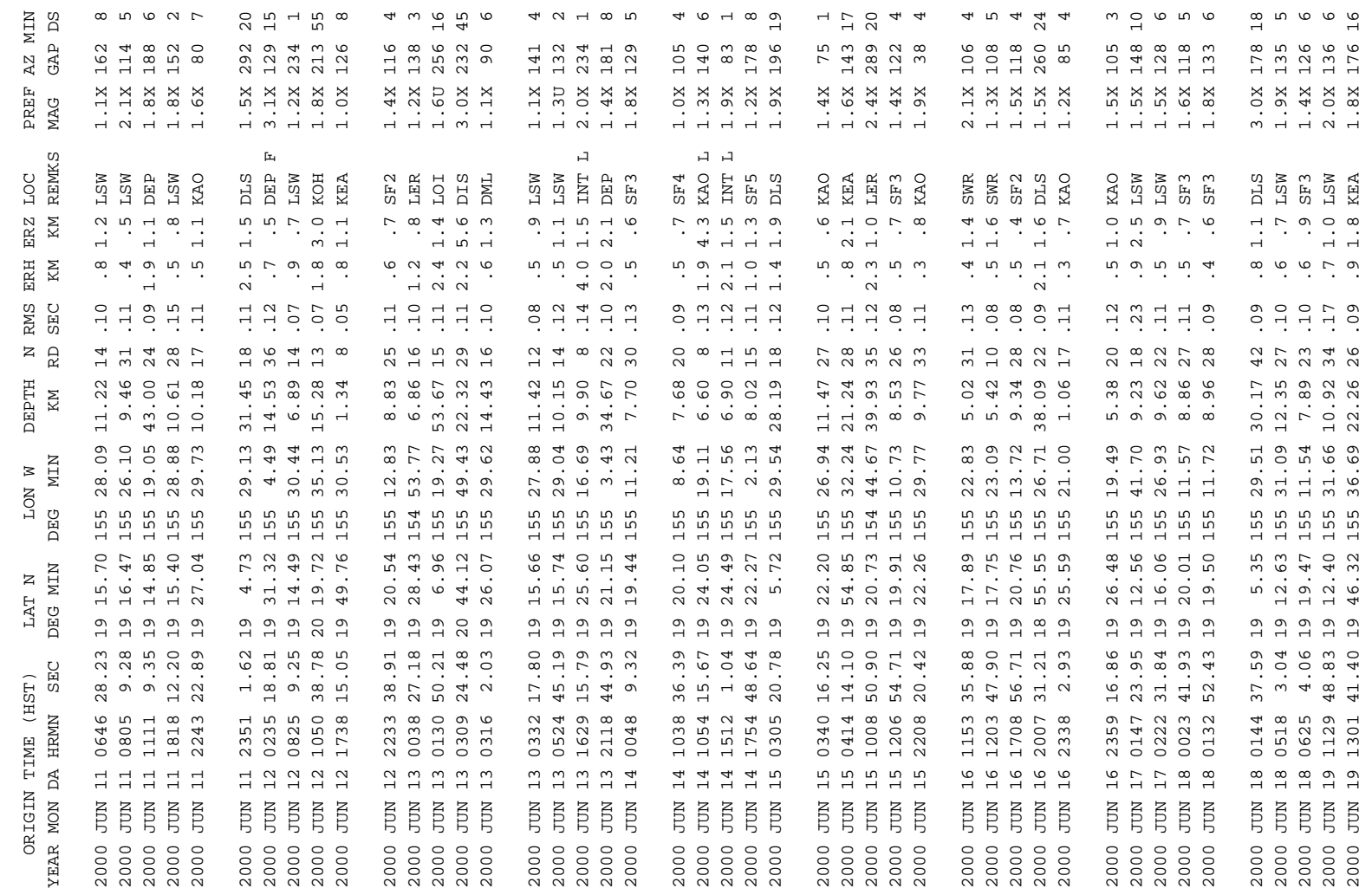

\begin{tabular}{|c|c|c|c|c|c|c|c|c|}
\hline 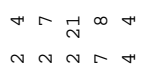 & $\begin{array}{l}m \sigma r N N \\
N \sim m a n\end{array}$ & 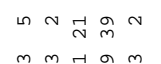 & $\begin{array}{l}\curvearrowleft \curvearrowleft \curvearrowleft \curvearrowleft N \\
\forall \neg m \Gamma-r\end{array}$ & 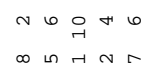 & 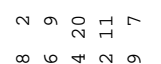 & 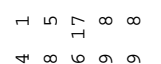 & $\begin{array}{l}\text { mognr } \\
\text { ognän }\end{array}$ & $\begin{array}{c}0 \pi+\pi) \\
n \rightarrow \infty\end{array}$ \\
\hline$\stackrel{\pi}{\Rightarrow}$ & 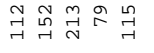 & 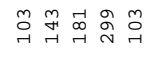 & $\underset{\mathcal{A}}{\tilde{N}} \stackrel{-1}{r}$ & 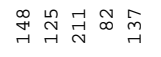 & 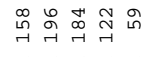 & 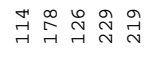 & 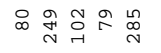 & $\stackrel{m}{\sim} \underset{N}{\infty} \infty \underset{\sim}{\infty} \underset{\sim}{\stackrel{N}{N}}$ \\
\hline 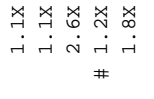 & 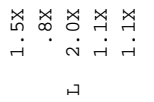 & 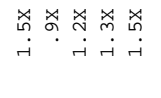 & 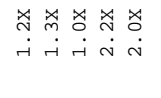 & 促 & 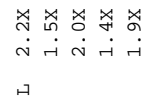 & 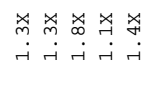 & 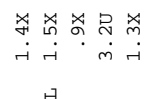 & 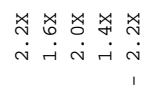 \\
\hline 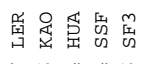 & 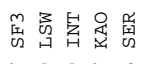 & 总总䍃苔意 & 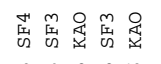 & 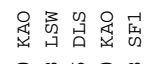 & 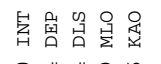 & 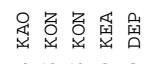 & 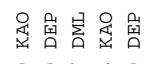 & 总兽总是 \\
\hline & ค.ด.ร.ค & 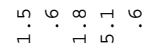 & & . & 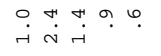 & & نं & . \\
\hline$n$ & 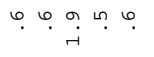 & 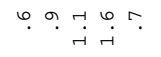 & & & 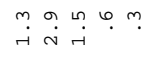 & ت. ت & $\dot{i}$ & \\
\hline ㄱํ ㅇํำ 구 & 웅ำ 웅. & ํํำ & $\vec{y}$ & 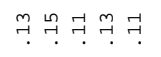 & 훙ㅇํㅇํ. 구 우 & $\because \approx m m$ & 우 웅구 구 공 & \\
\hline$\stackrel{n}{\rightarrow} \stackrel{9}{9}$ & 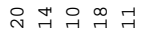 & 곡 & 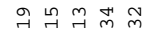 & ㅁำ & 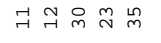 & $\stackrel{\infty}{\sim} \underset{\sim}{\sim} \stackrel{\sim}{\rightarrow} \underset{\sim}{N}$ & $\vec{N}^{\sigma}$ & $\stackrel{\circ}{\sim}$ \\
\hline 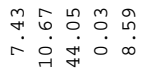 & 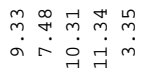 & 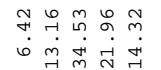 & 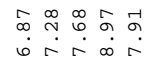 & 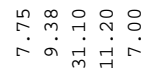 & 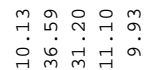 & 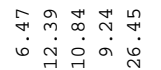 & 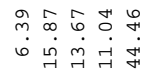 & \\
\hline & 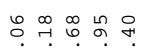 & 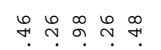 & $\stackrel{0}{?} \stackrel{\infty}{\infty}$ & 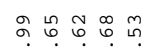 & 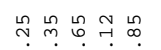 & 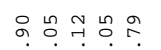 & 웅 & $\stackrel{?}{?}$ \\
\hline & $ت \dot{1}$ & $\stackrel{\sim}{\sim} \stackrel{\infty}{N}$ & $\dot{ت} \dot{0}$ & $\dot{\theta} \dot{\dot{\theta}}$ & 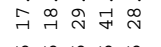 & 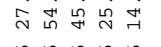 & & \\
\hline 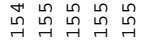 & 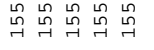 & 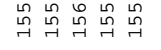 & 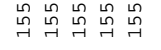 & 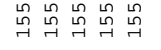 & 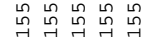 & 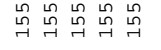 & 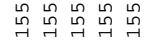 & 怘点总虽出 \\
\hline & gên & 实出 & . & : & $\stackrel{n}{n}$ & 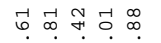 & 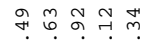 & 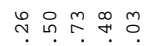 \\
\hline & $\stackrel{i}{i}$ & $\stackrel{\infty}{\sim} \stackrel{\infty}{\sim}$ & 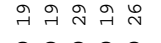 & 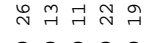 & $\sigma \stackrel{m}{m} \underset{N}{\infty}$ & & & \\
\hline & 90 & 웅요 & 을 & ๑ேの & $\sigma a$ & $g 9$ & $\Rightarrow \sigma$ & $\sigma \sigma$ \\
\hline & & & & & ? & $\vec{b}$ & 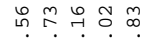 & \\
\hline & $\stackrel{\leftrightarrow}{N}$ & $\nexists$ & $\stackrel{\infty}{\infty}$, & 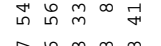 & 구 & ने & $a$ & $\stackrel{\infty}{\sim} \stackrel{\infty}{\sim}$ \\
\hline & & & & & & & & \\
\hline & & & & & & & & \\
\hline & & & 营 & & & & 总总 & \\
\hline & & & & & & : & na & \\
\hline
\end{tabular}




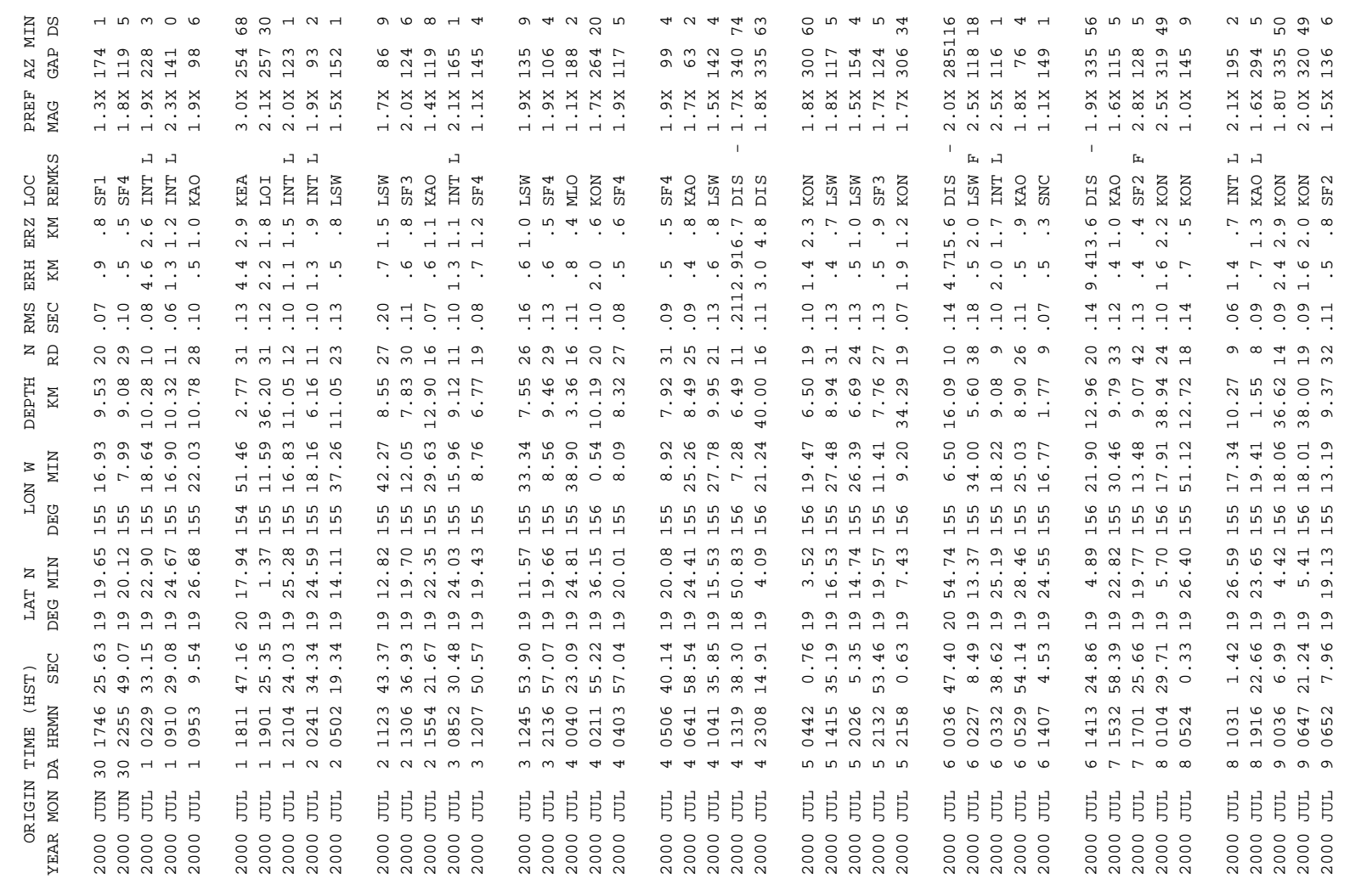

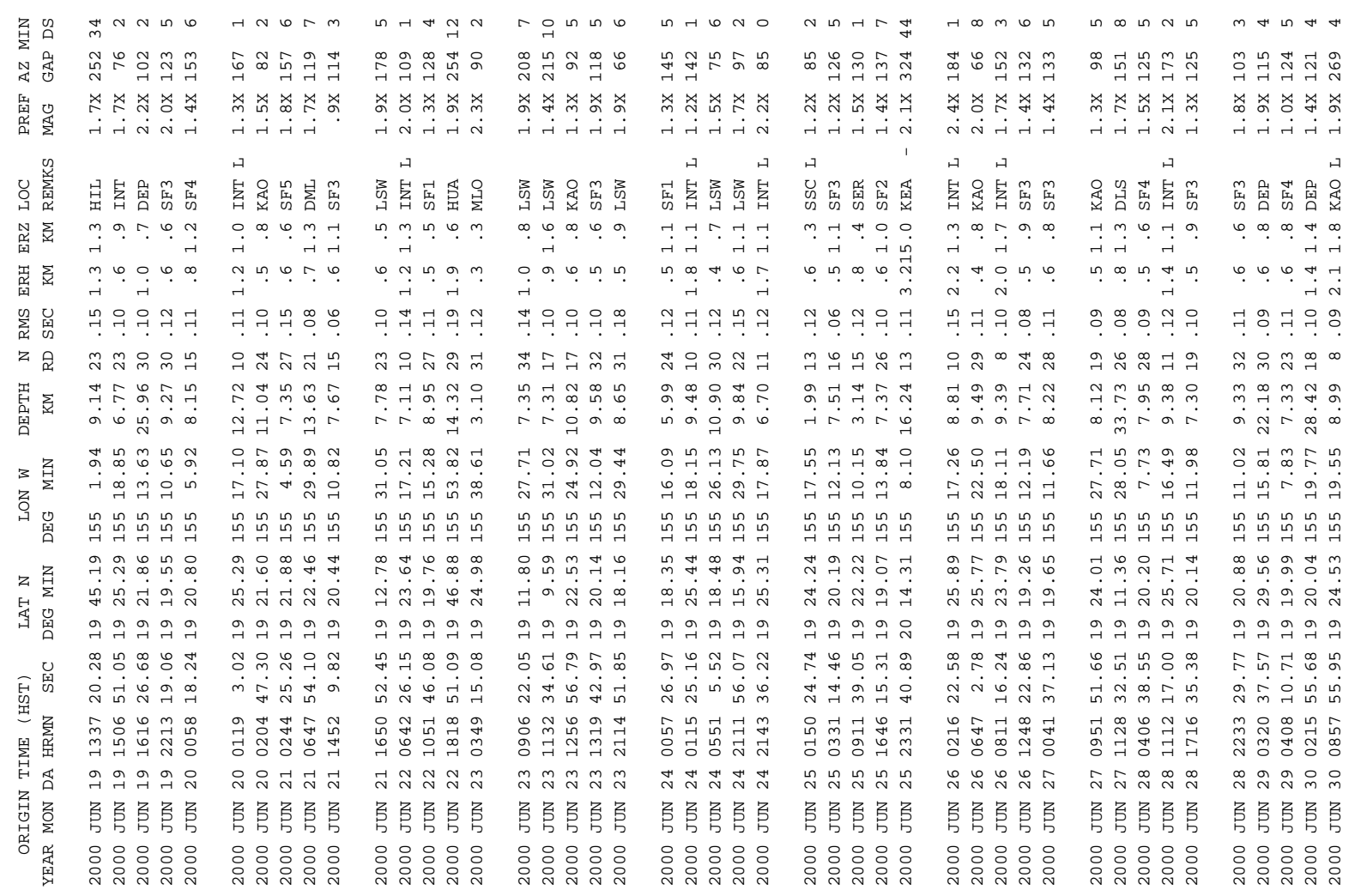




\begin{tabular}{|c|c|c|c|c|c|c|c|c|}
\hline 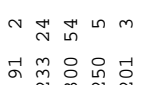 & 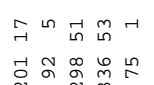 & 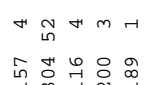 & 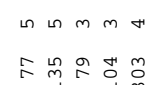 & 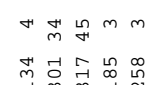 & 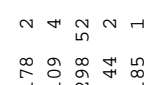 & 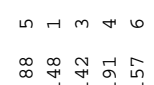 & 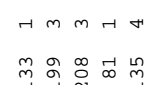 & 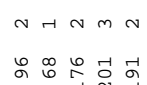 \\
\hline & 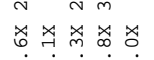 & 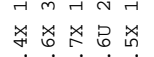 & 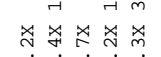 & 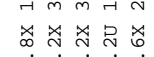 & 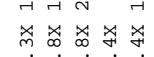 & 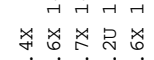 & 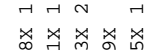 & - \\
\hline$\dot{H} \dot{N} \dot{N} \dot{N}$ & $\dot{r} \dot{r} \dot{\omega} \dot{\sim} \dot{\omega}$ & & & & & $\dot{H} \dot{\sim} \dot{\sim} \dot{\sim}$ & & \\
\hline 营番照 & 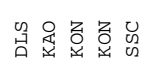 & z & & 要 & 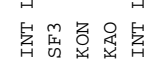 & 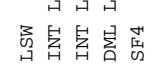 & 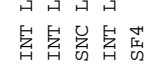 & 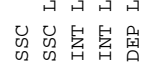 \\
\hline 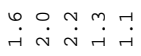 & 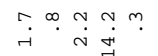 & 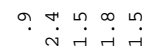 & 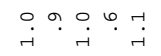 & $\begin{array}{l}\text { n? } \\
\dot{i}\end{array}$ & 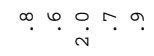 & 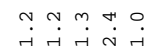 & 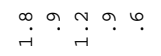 & $\because$. \\
\hline 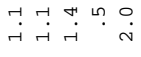 & 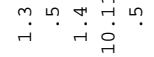 & 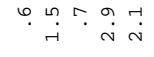 & 『. ". ". & 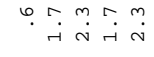 & ה & 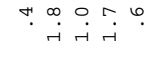 & 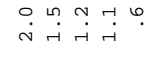 & ?. \\
\hline ๆ & 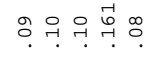 & 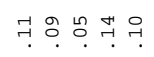 & 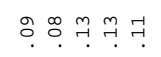 & 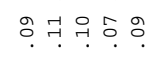 & 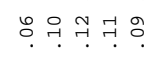 & ๆ ำ ㄱำ & 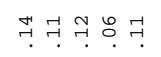 & $\stackrel{0}{\infty} \stackrel{m}{c}$ \\
\hline$\infty \underset{\sim}{\infty} \stackrel{\sim}{\sim} \sigma \vec{F}$ & $\vec{N} \stackrel{\infty}{\sim} \stackrel{\infty}{\sim} \vec{N} \vec{\sim}$ & $\stackrel{\mathbb{N}}{\sim} \stackrel{\mathbb{N}}{\sim} \vec{F}^{-}$ & $\stackrel{\mathscr{N}}{\sim} \underset{\sim}{\mathbb{N}} \stackrel{\sim}{\sim} \infty$ & $\stackrel{\sim}{N} \vec{N} \stackrel{\circ}{\circ}$ & 욤 $\mathrm{m}$ & $\stackrel{\sim}{\sim} \underset{\sim}{\circ}-\infty \vec{N}$ & 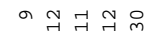 & 두욱 국 \\
\hline 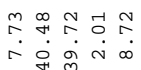 & 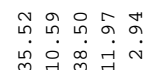 & 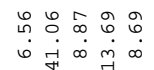 & 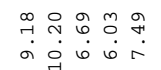 & & 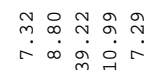 & 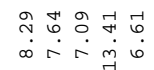 & & 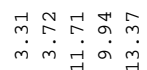 \\
\hline$\sim m p$ & 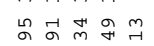 & i & . & & & in & $n$ & \\
\hline & & $6 \%$ & $\circ$ & & & & & \\
\hline 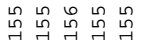 & 虽虽虽虽虽虽 & 虽虽虽虽虽虽 & 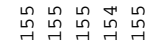 & 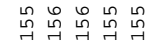 & 虽虽虽虽虽 & 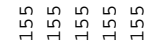 & 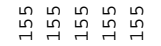 & 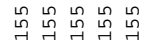 \\
\hline 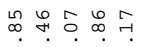 & 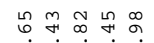 & 유 암. & $\stackrel{0}{?}$ & & ?. & & & $\pi$ \\
\hline$\stackrel{\dot{\sim}}{\sim} \dot{\sigma}$ & & ஸे & $\stackrel{\dot{\sim}}{\sim}$ & & $\stackrel{4}{2}$ & & & \\
\hline & & ने ने & $\overrightarrow{+}$ & & & & & $\sigma \sigma 0$ \\
\hline & & $N$ & & & & & & \\
\hline & & & & & & & & \\
\hline & & & & & & & & \\
\hline & & $\curvearrowleft$ & 足 & & & 도 & $=r$ & 드 \\
\hline & & (3) & 然 & & & & & 3 \\
\hline :ঃ: : : & bo & : : & 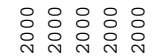 & & : : & 00 & 年 & :०: \\
\hline
\end{tabular}

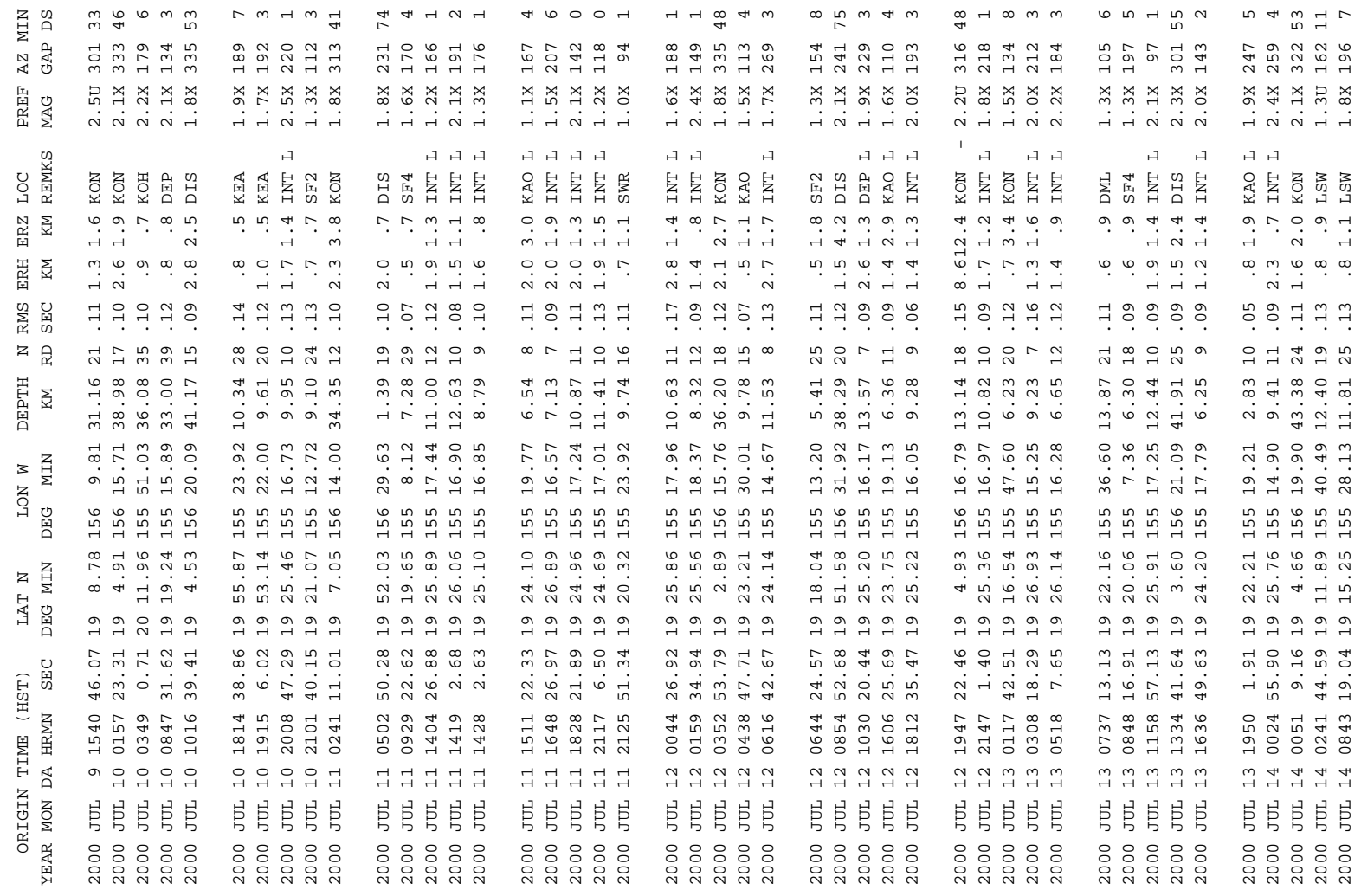




\begin{tabular}{|c|c|c|c|c|c|c|c|c|}
\hline$+m$ & $\infty \sim \underset{\sim}{N} \rightarrow r$ & $N \stackrel{N}{r} r N H$ & $H N \underset{\neg}{n} m$ N & $0440 \mathrm{n}$ & N $\backsim \backsim \vec{凸}$ & $H m \underset{\sim}{\infty} \in N$ & ar & $\theta a^{4}$ \\
\hline 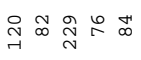 & 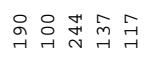 & 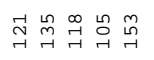 & N & 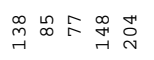 & 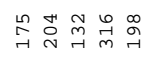 & 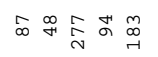 & 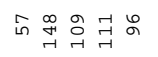 & 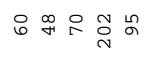 \\
\hline 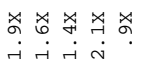 & 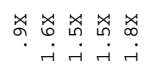 & 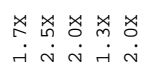 & 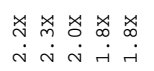 & 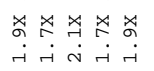 & 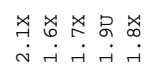 & 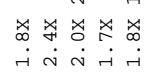 & 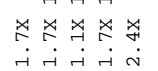 & 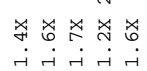 \\
\hline 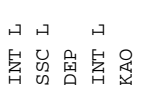 & 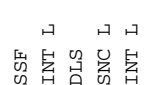 & 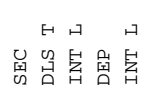 & 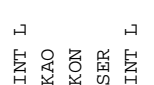 & 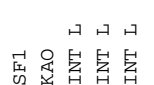 & 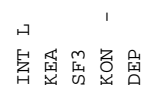 & 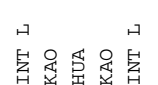 & 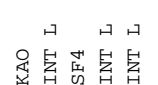 & 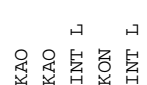 \\
\hline o. .7 & 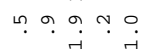 & 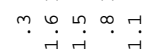 & & 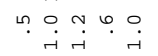 & & $\underset{r}{m} \cdot \stackrel{6}{m} \cdot \underset{j}{m}$ & ํ. & \\
\hline 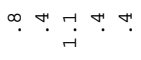 & & m. & 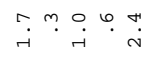 & 『. & 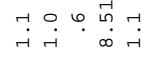 & 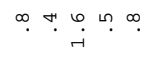 & $m$. & 4.7 \\
\hline 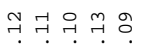 & 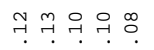 & 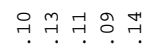 & 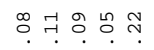 & 구 우 & 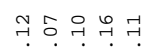 & 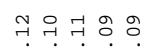 & 유 & \\
\hline$\stackrel{n}{\sim} \underset{\sim}{N} \stackrel{\sim}{m} \vec{m} \underset{N}{N}$ & 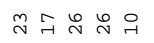 & 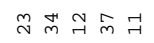 & 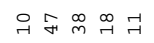 & 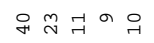 & 구 & $\stackrel{\sim}{\sim} \underset{\sim}{\sim} \stackrel{\mathrm{m}}{\mathrm{m}}$ & 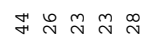 & \\
\hline 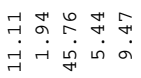 & 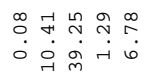 & 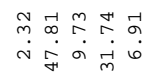 & 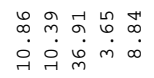 & 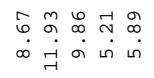 & 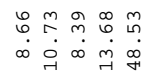 & 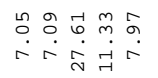 & & \\
\hline సે & 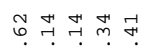 & 다. 아. & హె & స઼. & 유. m & $:$ & ตุ. & \\
\hline$\dot{\vec{े}} \dot{\vec{\sigma}}$ & $\begin{array}{l}\dot{\sim} \\
\rightarrow \\
\rightarrow\end{array}$ & 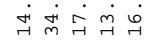 & 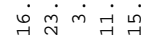 & $\dot{\vec{r}} \dot{\mathrm{N}}$ & $\dot{\vec{H}} \dot{N}$ & 我 & & \\
\hline 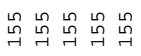 & 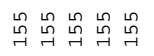 & 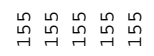 & 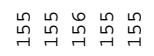 & 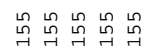 & 虽虽虽号虽虽 & 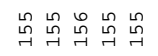 & 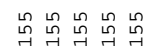 & 号吕号号 \\
\hline$\stackrel{\infty}{?} \stackrel{\sim}{?}$ & ㅇํำำ? & 도. & 아. 가. & mp & & & & \\
\hline & 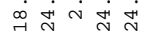 & $\dot{\sim} \sigma^{\circ}$ & 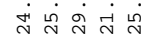 & 얼 & $\dot{\dot{v}}$ & & & \\
\hline & $\sigma a$ & & & & & & & \\
\hline no & œ & & in 10 & 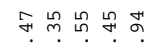 & & & & \\
\hline & & & & $\begin{array}{lll}\alpha \\
\end{array}$ & & & & \\
\hline 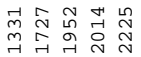 & 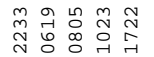 & 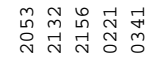 & 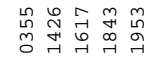 & 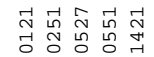 & 总 & $\begin{array}{l}\infty \\
\stackrel{\infty}{\infty} \stackrel{\infty}{0} \\
0\end{array}$ & $\tilde{\sim} \rightarrow \stackrel{\infty}{\infty}$ & \\
\hline & ม & & $\stackrel{\infty}{\sim} \stackrel{\infty}{\sim}$ & $\stackrel{\sim}{\sim} \underset{\sim}{\sim} \underset{\sim}{\mathbb{N}} \underset{\sim}{\sim}$ & & 芫员 & & \\
\hline & & & & 㞩㞩 & & & & \\
\hline : & 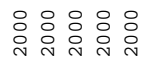 & 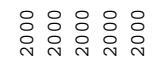 & 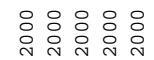 & 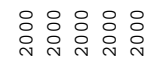 & 임 & & & \\
\hline
\end{tabular}

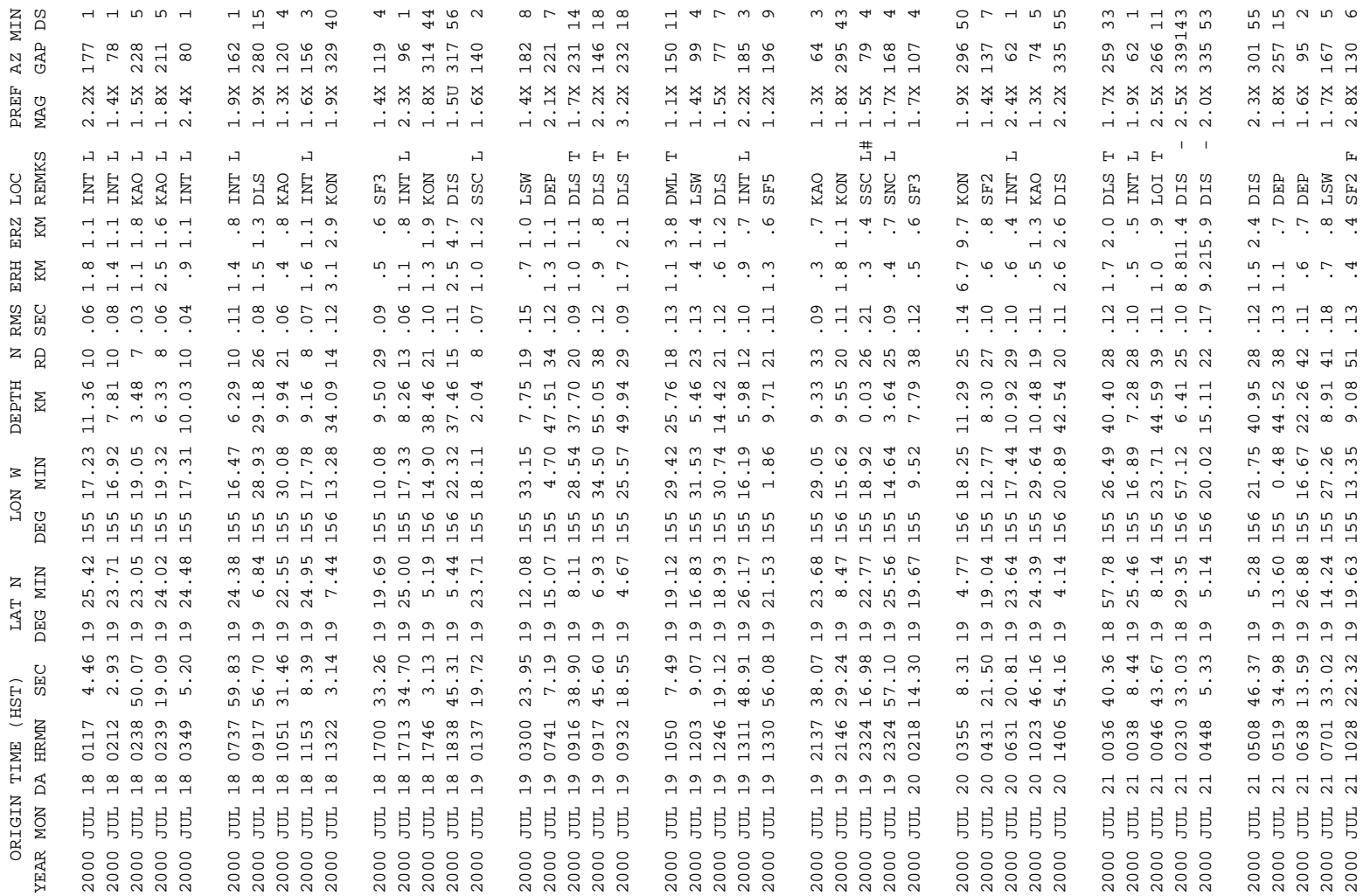




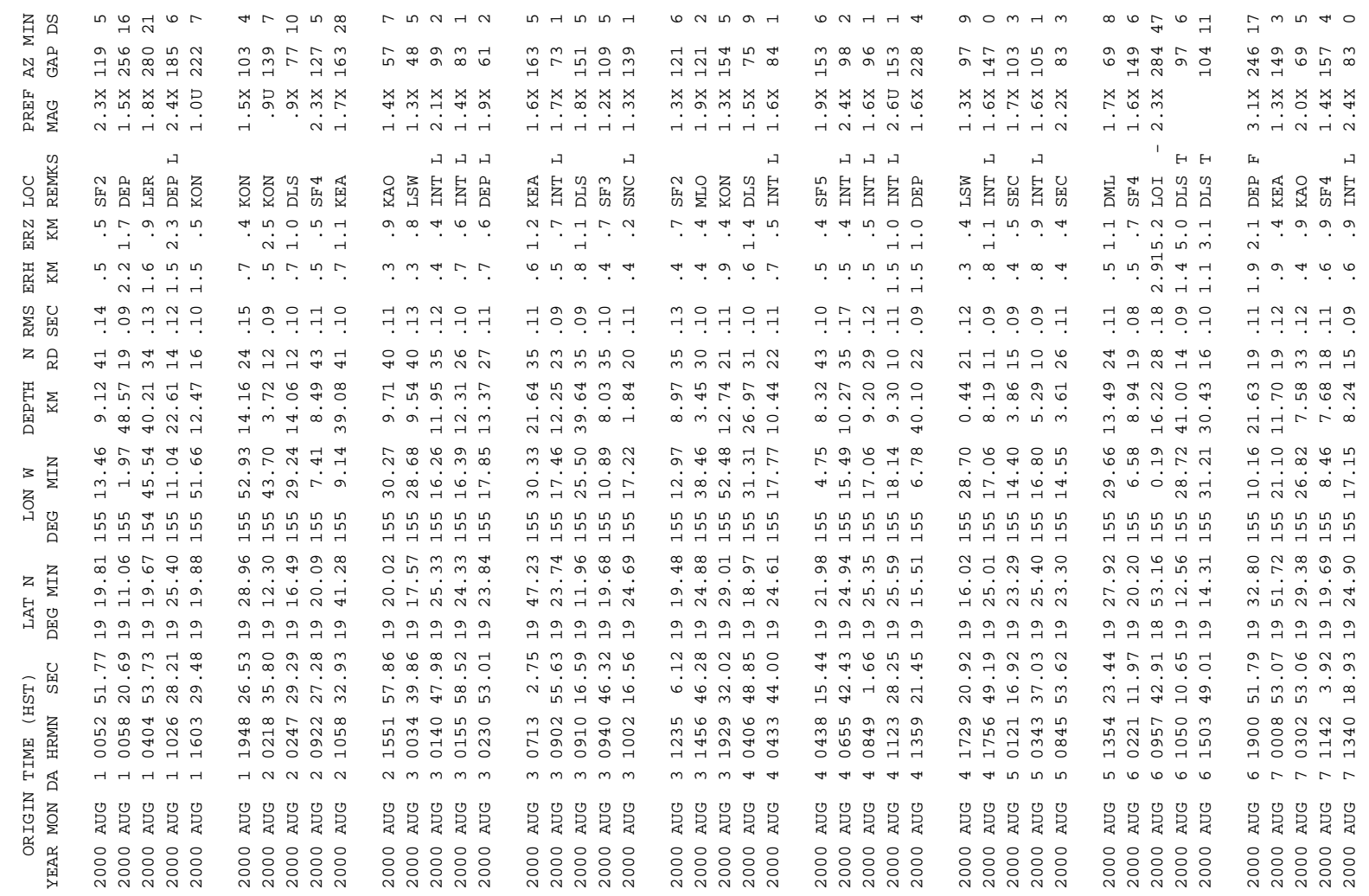

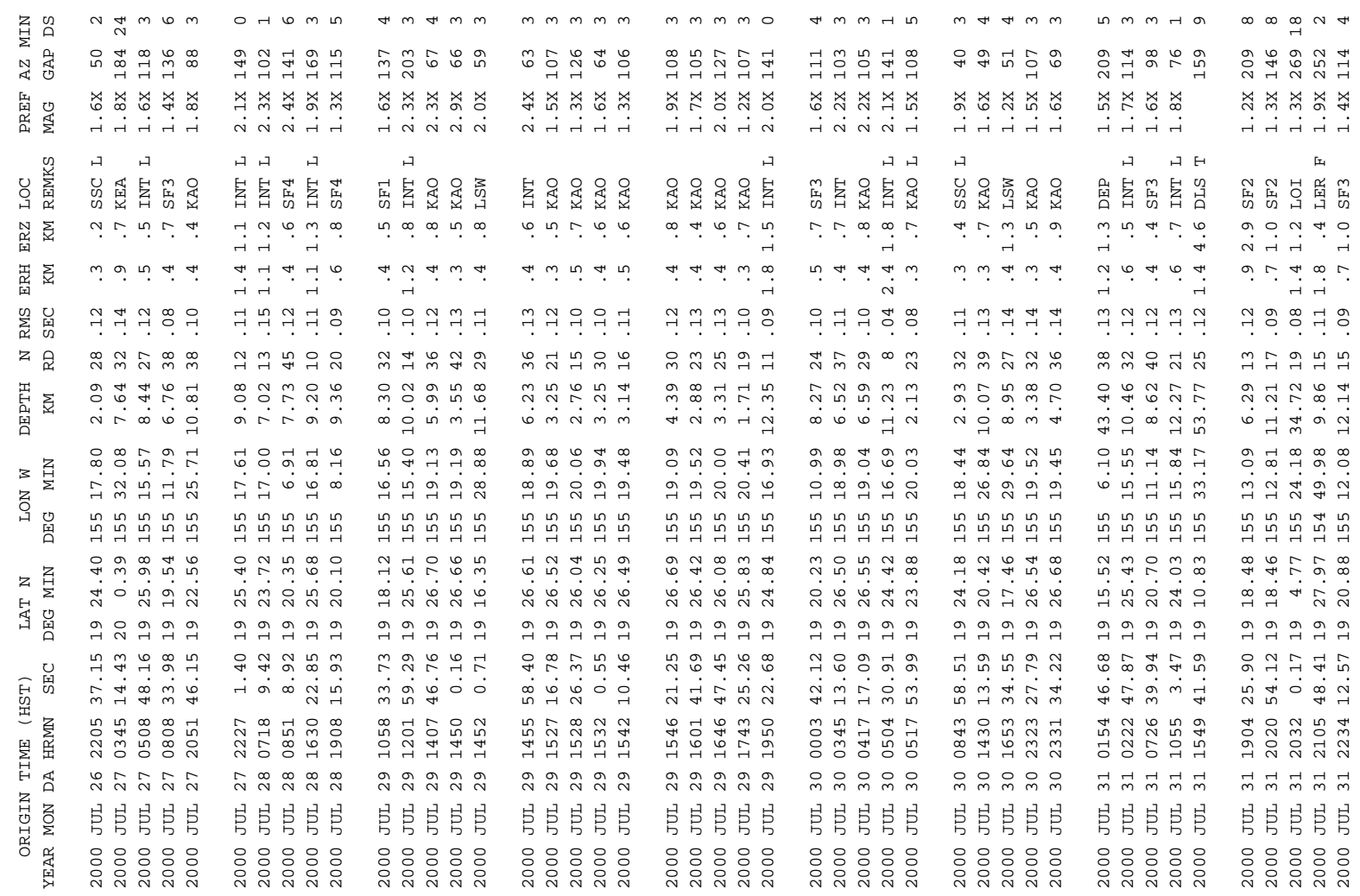




\begin{tabular}{|c|c|c|c|c|c|c|c|c|}
\hline $60+\pi N$ & $\neg 0$ & $\infty$ ๘ & $\stackrel{\circ}{\sim}$ & r & ने & N N & m & 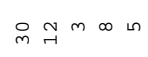 \\
\hline & 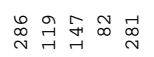 & & 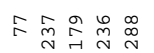 & 어료 & 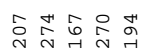 & 吕 $\underset{\sim}{\sim} \underset{\sim}{\sim}$ & & $\exists$ \\
\hline 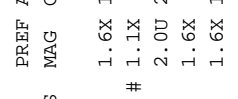 & 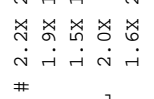 & 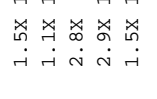 & 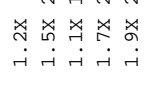 & 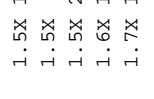 & 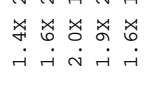 & 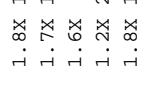 & 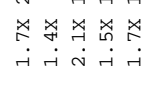 & $\vec{i}+\vec{i}$ \\
\hline & & $\begin{array}{l}\text { 壱 } \\
\text { c }\end{array}$ & 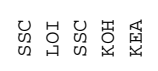 & 管 & 总是萃显畠 & & 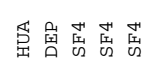 & 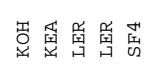 \\
\hline & $\begin{array}{ll}m \\
\dot{r} \\
\dot{i}\end{array}$ & $r$ & & & $r$ & & & \\
\hline & 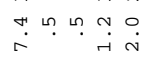 & $\because$ & r. & & 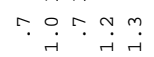 & $\therefore$. & ${ }^{\circ}$ & \\
\hline$\vec{I}$ & 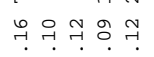 & 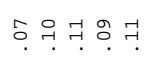 & : & 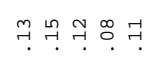 & 둑ㄱำ 군 움 & 웅ำ & & \\
\hline & $\vec{r} \mathrm{~m}$ & $\stackrel{\circ}{\text { i }}$ & 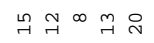 & & & & & \\
\hline & & & & & & & & \\
\hline & & & & & & & & \\
\hline & 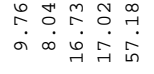 & 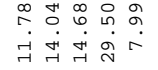 & & & & & & \\
\hline 虽 & 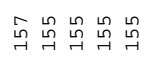 & 虽虽品品出出 & 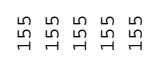 & 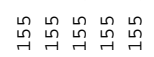 & 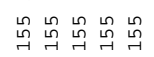 & 员品 & 㟔虽 & \\
\hline & 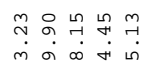 & & & & & & & \\
\hline & & & & & & & & \\
\hline & & & & & & & & \\
\hline & 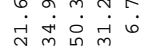 & & & & & & & \\
\hline & 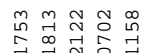 & 곡 & or & 로 & & & & \\
\hline & $\stackrel{\infty}{\sim} \stackrel{\infty}{\sim}$ & 웍ㅇ & & i & & & & \\
\hline & & & & & & & & \\
\hline 8 & 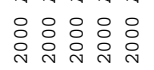 & $\begin{array}{l}0 \\
0 \\
0 \\
0\end{array}$ & 0 & \begin{tabular}{l}
0 \\
$O$ \\
0 \\
\hdashline
\end{tabular} & & & & \\
\hline
\end{tabular}

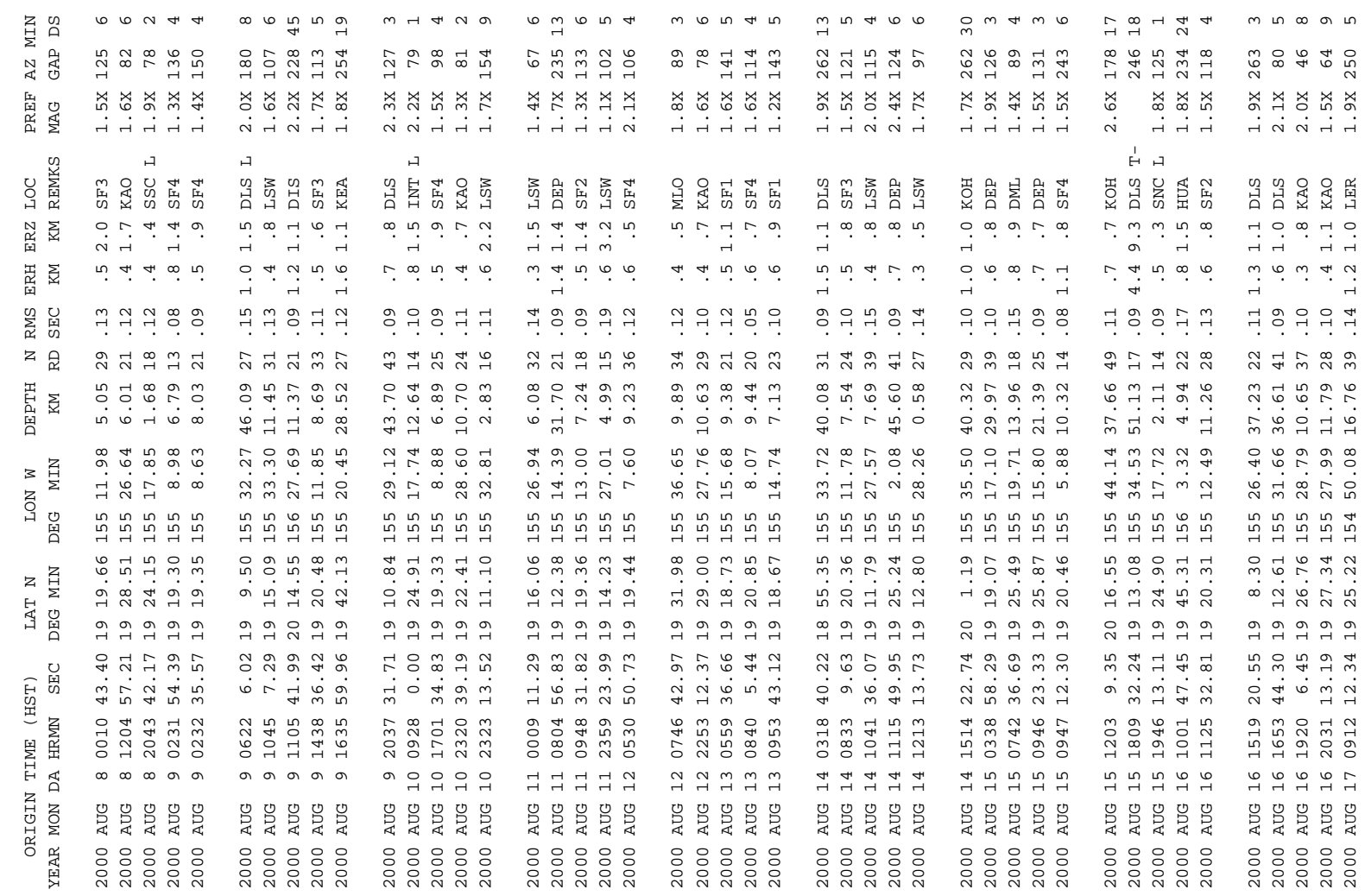




\begin{tabular}{|c|c|c|c|c|}
\hline & 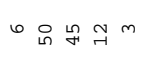 & $m+n \rightarrow r$ & $H+\infty \circ 6$ & $\neg \backsim \underset{N}{\circ} \backsim N$ \\
\hline & 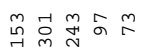 & $\approx \sigma \infty \Omega \underset{\sim}{ }$ & œ の ન & 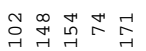 \\
\hline & 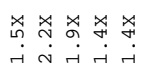 & 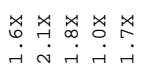 & 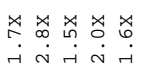 & 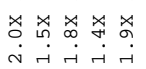 \\
\hline & & & 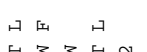 & \\
\hline & 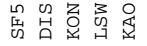 & 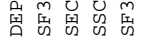 & 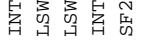 & 动 \\
\hline & 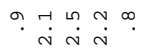 & 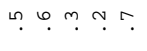 & 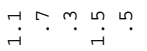 & 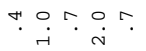 \\
\hline & 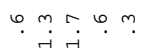 & ナ. サ. m. & 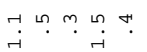 & $\varphi r \varphi \varphi$. \\
\hline & 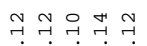 & 국영 옹우 & 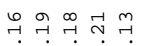 & 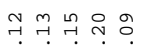 \\
\hline & $\stackrel{m}{m} \tilde{m} \approx \stackrel{\infty}{\sim} \vec{m}$ & $\vec{m} \ddot{m} \vec{N} \stackrel{\sim}{\sim} \stackrel{m}{m}$ & 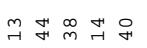 & 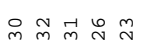 \\
\hline & 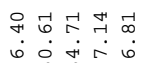 & 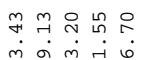 & 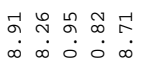 & 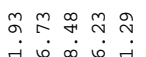 \\
\hline & 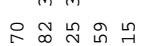 & 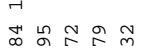 & 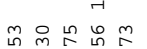 & $\underset{\sim}{M} \underset{\infty}{\infty}$ \\
\hline & 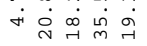 & 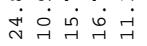 & $\dot{\sim} \underset{N}{ } \vec{m} \vec{\sim}$ & 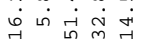 \\
\hline & 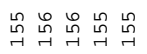 & 虽虽虽员员虽 & 怘怘虽虽虽 & 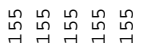 \\
\hline & 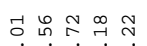 & 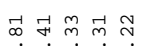 & 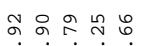 & 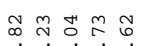 \\
\hline & 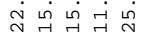 & $\dot{\sigma} \dot{\sim} \dot{\sim} \dot{\sim} \dot{\sim} \dot{N}$ & ন্ন & $\underset{N}{\sim} \dot{N} \stackrel{\sim}{\sim} \underset{\sim}{\stackrel{n}{N}}$ \\
\hline 造㒶 & 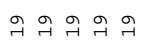 & 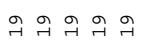 & 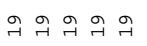 & 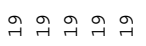 \\
\hline تص & 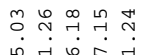 & 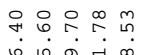 & 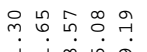 & mָ N. \\
\hline & 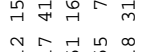 & 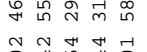 & 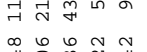 & \\
\hline & $\vec{n}$ & 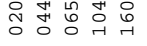 & 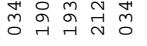 & \\
\hline & $\vec{m} \vec{m} ન ન-1$ & $\sim \sim \sim \sim N$ & $m m m m \sigma$ & 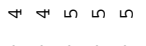 \\
\hline & 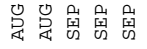 & 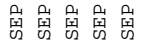 & 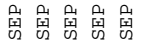 & 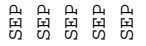 \\
\hline 秀 & 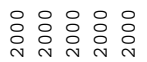 & 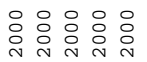 & $\begin{array}{l}: \\
: \\
:\end{array}$ & $\begin{array}{ll}0 \\
: \\
\vdots\end{array}$ \\
\hline
\end{tabular}

\begin{tabular}{|c|c|c|c|c|}
\hline$m \stackrel{\sim}{N} m 6 m$ & $\infty \vec{N} r m \rightarrow$ & $\underset{\sim}{\pi} m \infty \underset{\sim}{\sim} 0$ & $m \underset{\sim}{m} \underset{\sim}{\infty}+r$ & $m \sim m m a$ \\
\hline 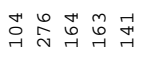 & 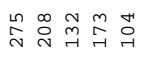 & 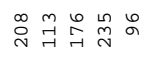 & 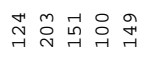 & 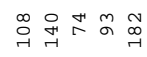 \\
\hline 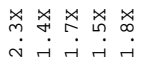 & 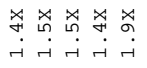 & 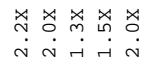 & 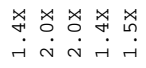 & 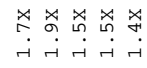 \\
\hline & 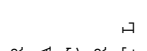 & & & \\
\hline 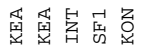 & 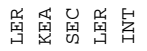 & 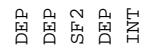 & 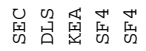 & 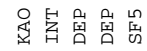 \\
\hline 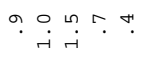 & 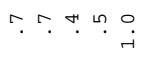 & 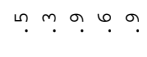 & 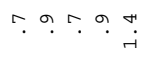 & • \\
\hline 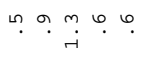 & 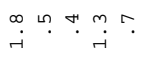 & • & †. $\varphi . \varphi$. & 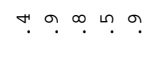 \\
\hline 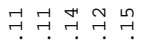 & 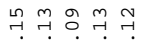 & 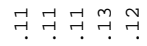 & 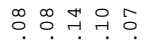 & ำ 욱 \\
\hline$\exists \stackrel{\circ}{\sim} \stackrel{\infty}{N}$ & $\tilde{F} \underset{\sim}{\sim} \stackrel{\infty}{\sim} \stackrel{\sim}{\sim} \underset{\sim}{\sim}$ & 욤도 & 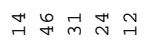 & $\stackrel{m}{\sim} \underset{\sim}{\sim} \stackrel{m}{m} \stackrel{m}{\sim}$ \\
\hline 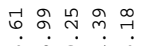 & 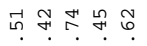 & 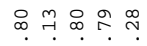 & 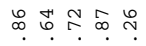 & 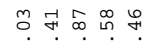 \\
\hline هं & $\dot{\sim} \dot{0} \dot{\sim} \dot{m} \infty \dot{O}_{\vec{H}}^{\dot{0}}$ & 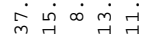 & 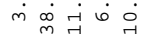 & $\dot{m} \underset{\sim}{\dot{\mathcal{F}}} \dot{m} \vec{m} \underset{\sim}{\vec{G}} \dot{n}$ \\
\hline 号 & क. & 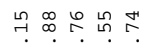 & 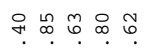 & 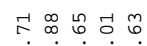 \\
\hline मे & 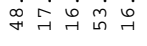 & 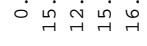 & 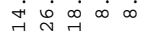 & \\
\hline 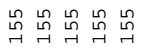 & 㟔出出虽出 & 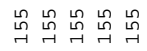 & 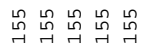 & 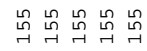 \\
\hline ก. ? & 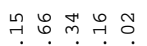 & 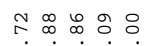 & 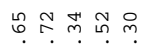 & చூ \\
\hline$\dot{\sim} \vec{\sim} \dot{\sim} \sim \dot{\sim}$ & $\dot{\sim} \dot{\sim} \dot{m} \underset{\sim}{\dot{N}} \dot{\sim} \stackrel{\dot{N}}{N}$ & $\dot{\vec{F}} \stackrel{\dot{N}}{\vec{f}} \dot{\vec{m}} \dot{\sim}$ & $\dot{\sim} \dot{\sim} \dot{\sim} \dot{m} \vec{\sigma}$ & 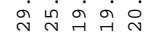 \\
\hline 악 & 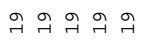 & 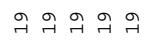 & 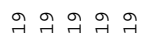 & 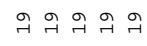 \\
\hline $2 \infty$ & 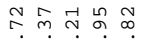 & 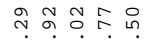 & 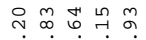 & 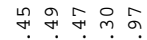 \\
\hline 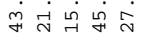 & $\dot{\sim} \dot{\sigma} \dot{\sigma} \dot{m} \dot{m} \infty \dot{m}$ & $\dot{m} \dot{m} \dot{b} \underset{\sigma}{\dot{\sigma}} \dot{\sigma}$ & 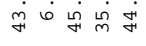 & 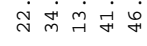 \\
\hline & 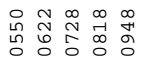 & 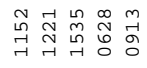 & 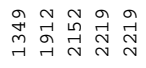 & $\stackrel{\infty}{\stackrel{\infty}{N}}$ \\
\hline & & & & \\
\hline & & & & \\
\hline & $: \circ$ & : : & 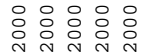 & : : : : \\
\hline
\end{tabular}

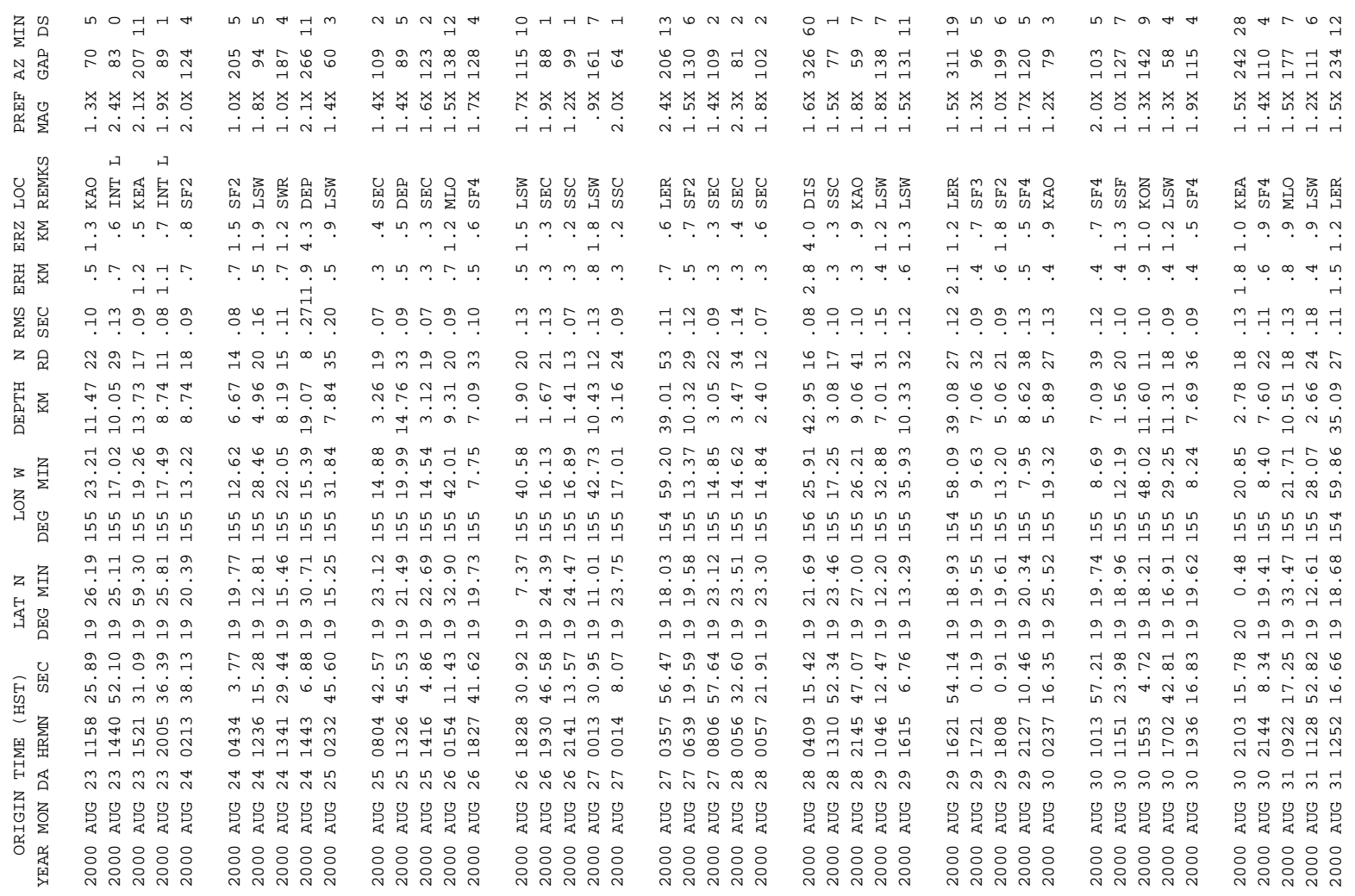




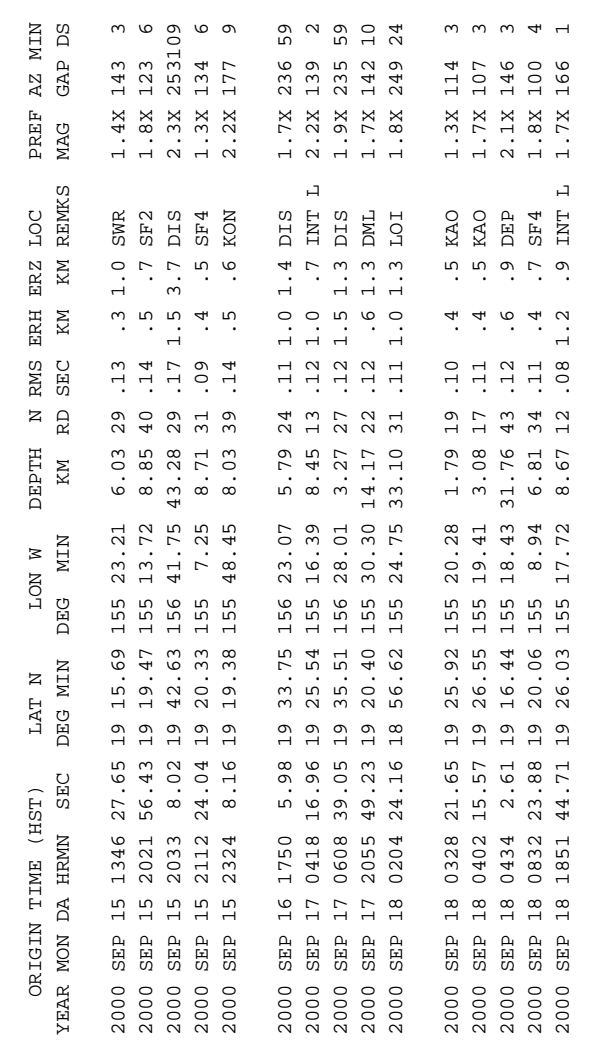

\begin{abstract}
monma
rrann

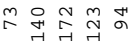

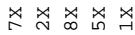

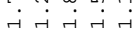

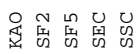

아. ग.

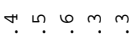

욱ㄱ 꿍용ㅇㅇ

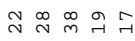

จิ

r $\dot{0} \dot{\infty} \dot{m} \dot{m}$

응요용ํํ

จं

总哭㽞嵒嵒

ఫౌ

ม่ำ

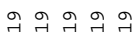

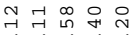

ते नें

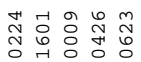

국ㄱㅇㅇㅅㅇㅇ

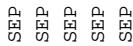

웅ㅇㅇㅇㅇㅇㅁ

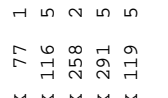

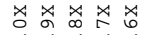

वंनंम

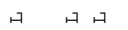

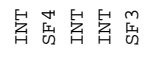

ఈ m.

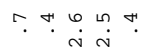

욱운우웅물

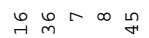

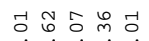

कें

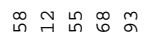

मे क्ष

虽虽品虽虽虽

닫ㅎํ워

중웡

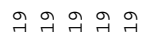

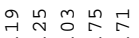

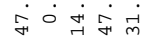

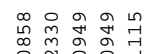

융तत

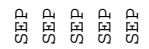

웅융영

m

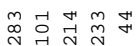

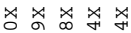

نं

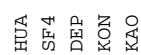

ㄱ. ㄴ.?ㄴ.?

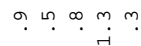

국ㄱㄱㄱㄱㄱ

웜ำ

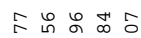

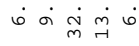

m

†ळ

虽嵒总总品

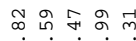

ช்

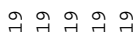

궁으의 유용

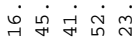

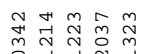

ำ Nำ

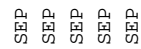

영융영
\end{abstract}

\begin{tabular}{|c|c|}
\hline 怘 & 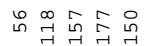 \\
\hline 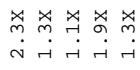 & 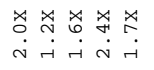 \\
\hline 预 & 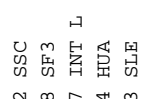 \\
\hline$\pi$ & 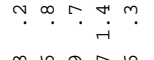 \\
\hline$\Rightarrow$ & 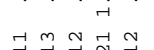 \\
\hline 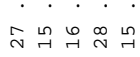 & 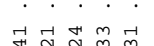 \\
\hline$n$ 우요 & 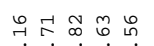 \\
\hline$\dot{b} \dot{\varphi} \dot{m} \dot{m}$ & 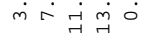 \\
\hline & $\vec{i} \underset{i}{\vec{H}}$ \\
\hline 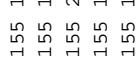 & 另员员员员 \\
\hline & 㘶这 \\
\hline & 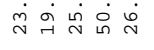 \\
\hline दी ने ने ने ने & $a \sigma$ \\
\hline$\stackrel{n}{\sim}$ & 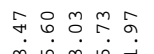 \\
\hline 5 & \\
\hline & \\
\hline & L \\
\hline & \\
\hline & \\
\hline
\end{tabular}

\begin{tabular}{|c|c|c|c|c|c|c|c|c|}
\hline 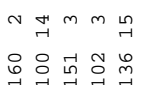 & 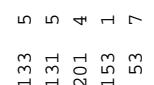 & 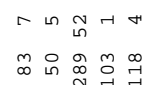 & 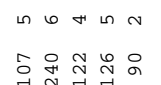 & 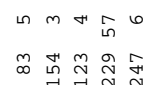 & 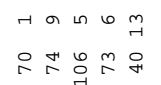 & 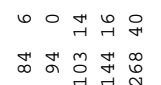 & 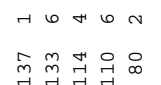 & 욤욤 \\
\hline نُ & 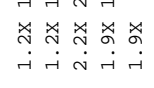 & 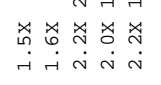 & 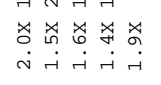 & 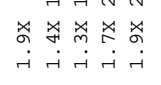 & 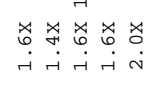 & 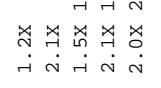 & 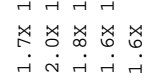 & 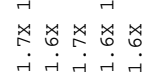 \\
\hline 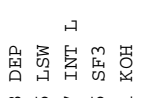 & 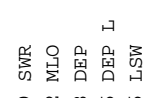 & 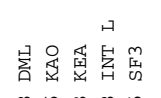 & 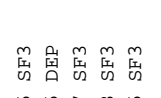 & 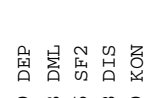 & 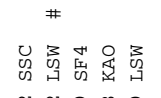 & 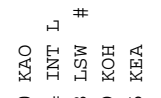 & 早总兽虫曾 & 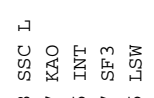 \\
\hline$\vec{i} \cdot \overrightarrow{0} \cdot 7$ & 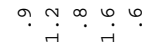 & 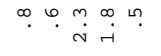 & & & & 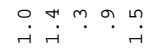 & & \\
\hline & $\stackrel{6}{i} \stackrel{m}{i}$ & 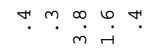 & & $\stackrel{\infty}{\infty}$ & 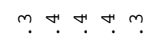 & & & \\
\hline 8 & 27 & 구 용 $\stackrel{\infty}{0}$ & 오. 굼 :ㅇํ. & 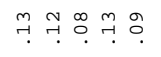 & 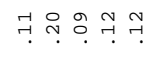 & ๆ $\stackrel{m}{?}$. & 의 & 의 \\
\hline 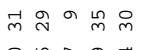 & 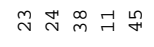 & 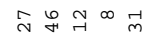 & $\stackrel{\infty}{m} \cap \stackrel{\infty}{\sim} \stackrel{\infty}{\sim} \stackrel{\infty}{\sim}$ & $\prod_{m}^{\infty} \vec{m} \sim \stackrel{\infty}{\sim} \stackrel{\pi}{*}$ & N⿴囗十心 & 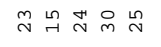 & 욤 & 웅 \\
\hline & 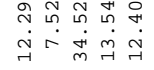 & 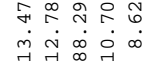 & 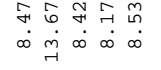 & 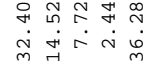 & 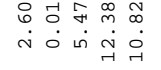 & 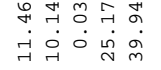 & 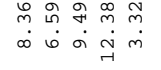 & 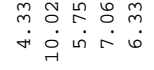 \\
\hline & 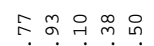 & 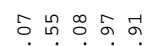 & 울 ำ 농 원? & Jా & 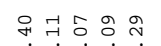 & 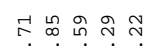 & 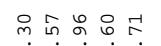 & 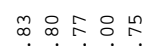 \\
\hline & $\dot{\vec{\prime}} \stackrel{0}{\sim}$ & $\dot{m}$ i m in 0 & ت어격 & 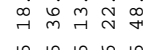 & $\vec{j} \dot{m} \sigma^{\circ} \dot{m}$ & 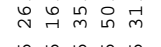 & م่ & $\dot{0}$ \\
\hline 员员 & 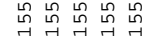 & 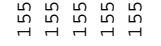 & 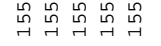 & 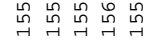 & 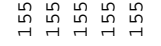 & 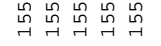 & 虽哭嵒 & 蛊 \\
\hline & ำ ㅇำ & 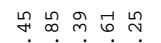 & का & 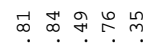 & $\rightarrow$ & 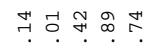 & $\vec{F}$. & $\stackrel{m}{\rightarrow}$ \\
\hline & $r_{-\infty}^{\infty}$ & N & - & 0 & $\stackrel{\infty}{\sim} \stackrel{\infty}{\rightarrow}$ & & N & - \\
\hline & ने & ने: & 욕 & 윅 아 & ने ने & & & 욱 \\
\hline & & $F$ & $\vec{N}$ & \% & $\stackrel{\leftrightarrow}{\sharp}$ & & & \\
\hline & & & \begin{tabular}{|l} 
a \\
$\dot{4}$
\end{tabular} & लि内 & $\stackrel{i}{i}$ & & & \\
\hline & & & 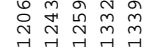 & 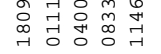 & 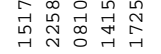 & 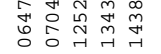 & & \\
\hline & & & & & 각구 국 & & & f \\
\hline & & & 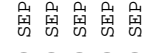 & 畄盖量 & 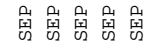 & 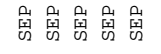 & 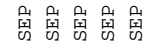 & s. \\
\hline & & & 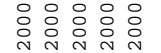 & & : : $: \circ: \circ:$ & & 年 & 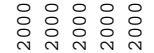 \\
\hline
\end{tabular}




\begin{tabular}{|c|c|c|c|c|c|c|c|c|}
\hline 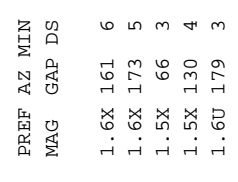 & 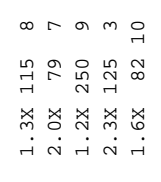 & 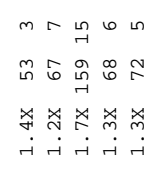 & 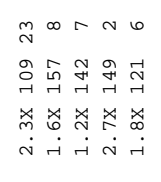 & 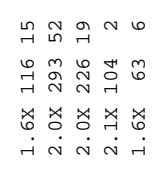 & 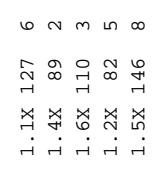 & 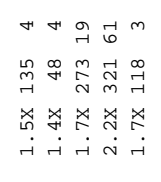 & 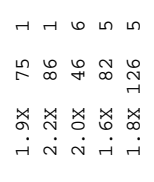 & 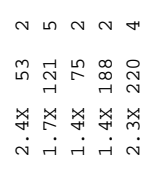 \\
\hline 画 & 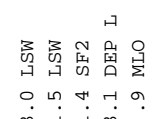 & 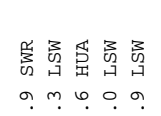 & 啰 & 4 & 荺骂 & 4 & & 前兽 \\
\hline ?. ๑. ค. .. & & & & & & & 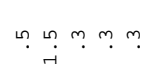 & m. \\
\hline$\stackrel{m}{?} \stackrel{7}{?}$ & $\stackrel{\infty}{?} \stackrel{\infty}{\square}$ & $m o \sim \infty$ & $\theta 0$ & 구 ํำ 구 군 & 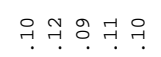 & & નન ન્옹 ન્ન & \\
\hline & $\sim \infty \sigma \infty$ & & & & & & & \\
\hline${ }_{0 \rightarrow-1}^{m}$ & 667 & $\dot{\sigma \infty} \dot{\vec{j}} \dot{\sim} \dot{\sigma}$ & & & & & & \\
\hline & & & & & & & & \\
\hline & & & & 6 & 总吕 & -1 & مُ & \\
\hline & & & & & & & & \\
\hline & & & & & & & & \\
\hline & & & & & & & & \\
\hline & & & & & & & & \\
\hline & & & & & & & & \\
\hline & & & & & 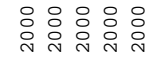 & & 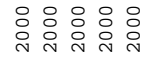 & \\
\hline
\end{tabular}

\begin{tabular}{|c|c|c|c|c|c|c|c|c|}
\hline$N \infty \approx 0$ & 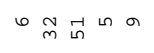 & 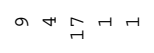 & 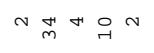 & 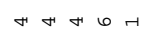 & $H ન+\mathbb{N}$ & ৮ & erthn & 0606 \\
\hline 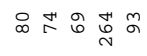 & 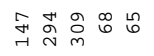 & 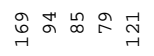 & 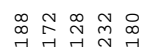 & 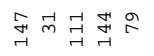 & 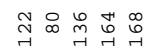 & 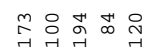 & 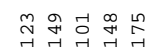 & \\
\hline 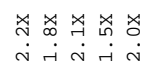 & 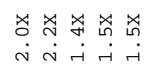 & 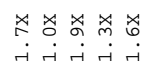 & 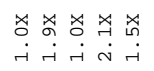 & 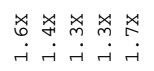 & 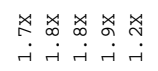 & 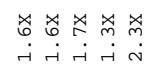 & 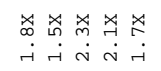 & \\
\hline 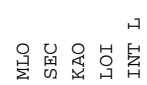 & 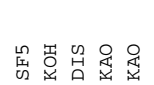 & 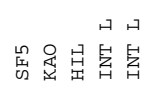 & 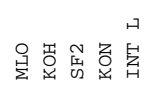 & 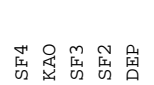 & 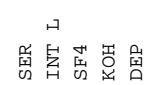 & 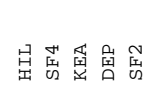 & 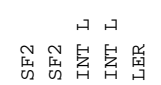 & 岁岁台 \\
\hline P. & 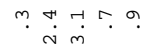 & 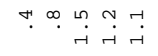 & 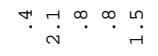 & $\vdash$. & 車 & & & \\
\hline$:_{4}^{6}$. & 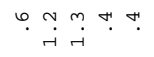 & 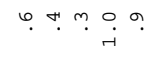 & r. & . & ن & ${ }^{\circ}$ & मे & - \\
\hline 굴 국욱 & નㅇํำำ & ஓㅇํㅇํำ 굼 & 국우 구ํ 운 & 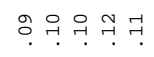 & 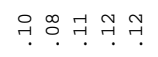 & $\exists \Rightarrow \underset{m}{\exists}$ & 함유ำ & \\
\hline 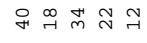 & $\vec{\sigma} \underset{\sim}{N} \stackrel{\sim}{N} \mathbb{m} \vec{m}$ & 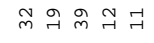 & 엄 웜 & 요 $\underset{m}{\infty} \stackrel{\infty}{\sim} \underset{\sim}{\sim} \stackrel{D}{m}$ & 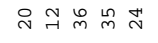 & in & 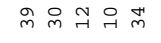 & \\
\hline r. & 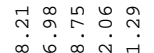 & 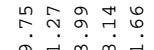 & 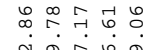 & $\approx$ & m & $\pi$ & 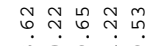 & \\
\hline & $\infty^{\infty}$ & ${ }^{a}=$ & 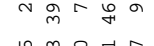 & 3 & & & & \\
\hline 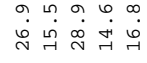 & mis & 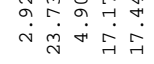 & 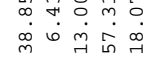 & 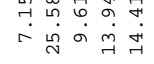 & 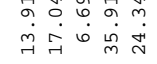 & & & की \\
\hline 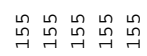 & 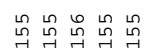 & 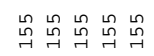 & 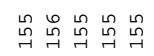 & 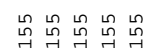 & 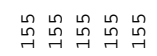 & 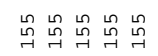 & 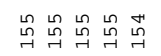 & 虽虽吕品 \\
\hline \% : & ?. & 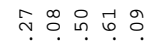 & & & & & & \\
\hline$i \stackrel{n}{N}$ & $\hat{N}$ & $\stackrel{N}{N} N \underset{m}{N} \underset{N}{N} \stackrel{n}{N}$ & & s. & $n$ & & & \\
\hline ने ने & 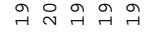 & ને ને ને ને ને ને & $\Rightarrow a$ & $\sigma$ & $\theta \sigma$ & th & 9 & \\
\hline $5 \%$ & 帒? & की & & 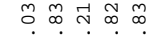 & 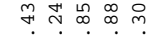 & & & \\
\hline & $N$ & N $\stackrel{\sim}{N}$ & & $\stackrel{m}{m} \stackrel{\sim}{N}$ & & & & \\
\hline 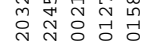 & 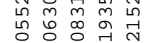 & 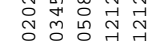 & & $7=$ & & & & \\
\hline$\stackrel{\circ}{N}$ & $\hat{\sim}$ & $\stackrel{\infty}{\sim} \stackrel{\infty}{\sim}$ & & s & & & & \\
\hline & 堌箅 & 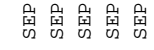 & 筧 & 葸葸 & 崽莒 & o g & & \\
\hline 家: & ch & 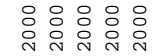 & & ${ }_{N}{ }_{N}$ & 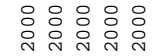 & & 等 & \\
\hline
\end{tabular}




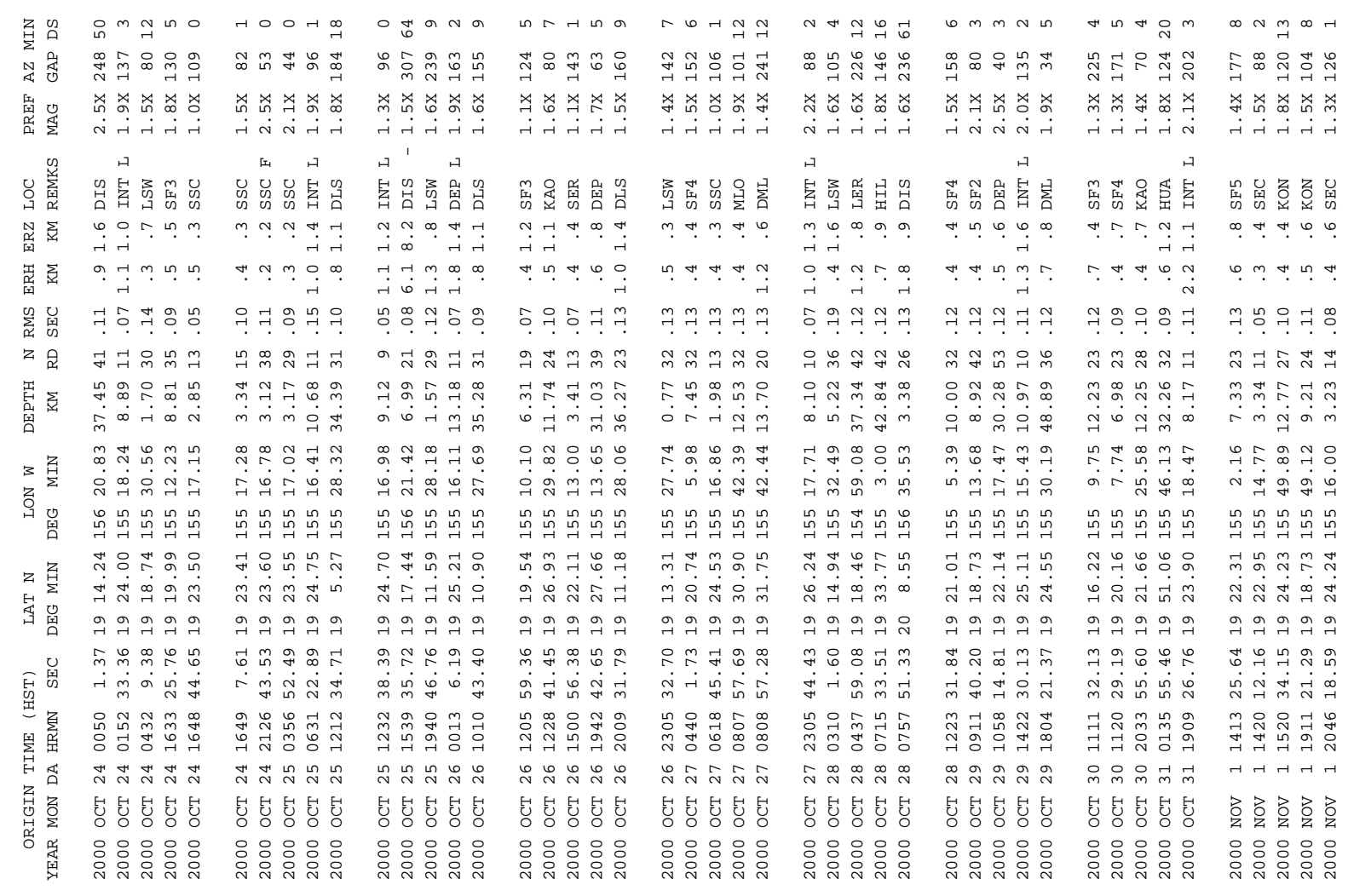

\begin{tabular}{|c|c|c|c|c|c|c|c|c|}
\hline$\vec{\gamma} \sigma$ & $m \backsim \pi \curvearrowleft$ & m & †ナ & $0 \infty r$ & $6 \pi 6$ & 40 & $\sigma$ & a \\
\hline 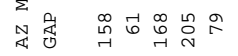 & ล์ト & 学学 & 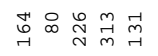 & 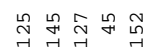 & 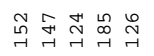 & 离 & 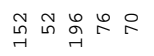 & 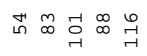 \\
\hline 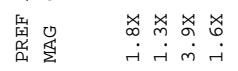 & 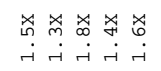 & 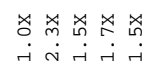 & 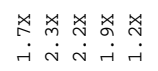 & 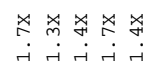 & 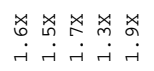 & 至 & 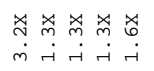 & ঝ \\
\hline & & & 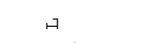 & & & & & \\
\hline 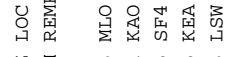 & 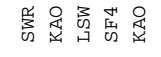 & 送总总罢 & 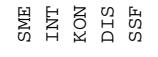 & 迅荠 & 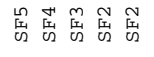 & & 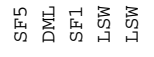 & \\
\hline$\stackrel{n}{i} \stackrel{\infty}{\infty}^{\infty}$. & 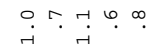 & & 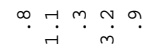 & & & & $\vec{j}+r$ & \\
\hline & & & 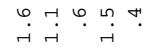 & & & & & \\
\hline 운 국 군 & 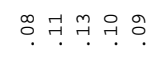 & 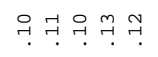 & 요 & 4 & $n$ & & $\stackrel{?}{? !} \stackrel{?}{?}$ & \\
\hline$\vec{m}$ & $\approx$ & n & $\because \underset{m}{ } \because$ & & & & & \\
\hline & 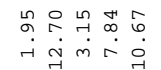 & & & & & & & \\
\hline & & & & & & & & \\
\hline & & & $\dot{m} \dot{ }$ & & & & & \\
\hline 足 & 怘虽 & مُ & 嵒吕 & & & & 㗊 & \\
\hline & & & & & & & & \\
\hline & & & & & & & & \\
\hline & & & & & & & & \\
\hline & & & & & & & & \\
\hline & & & & & & & & \\
\hline & 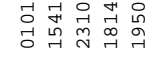 & & & & & & & \\
\hline & & & & & & & & \\
\hline & 苟获 & & 范获 & & & & & \\
\hline & & & 80 & & & & & \\
\hline
\end{tabular}




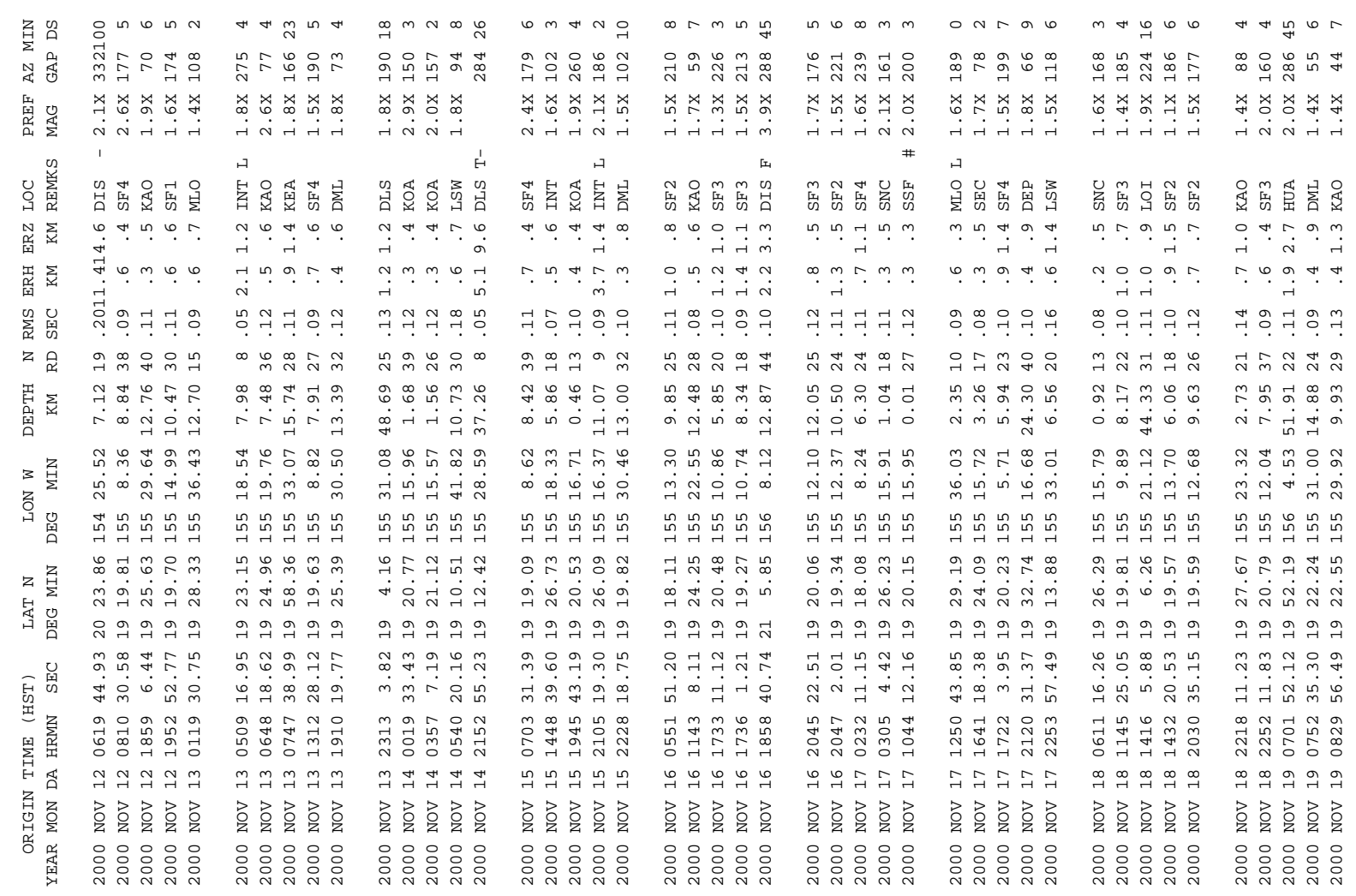

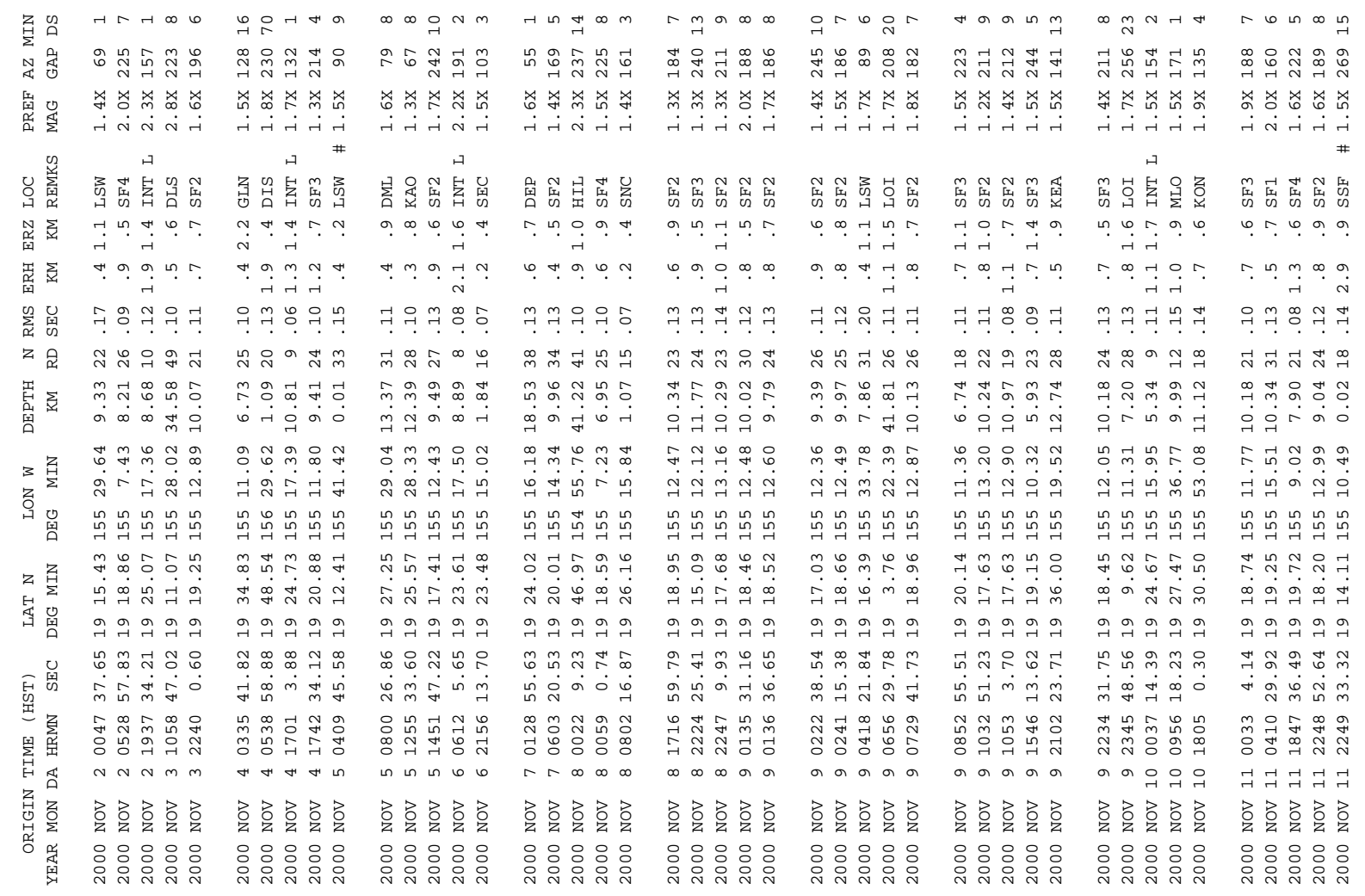




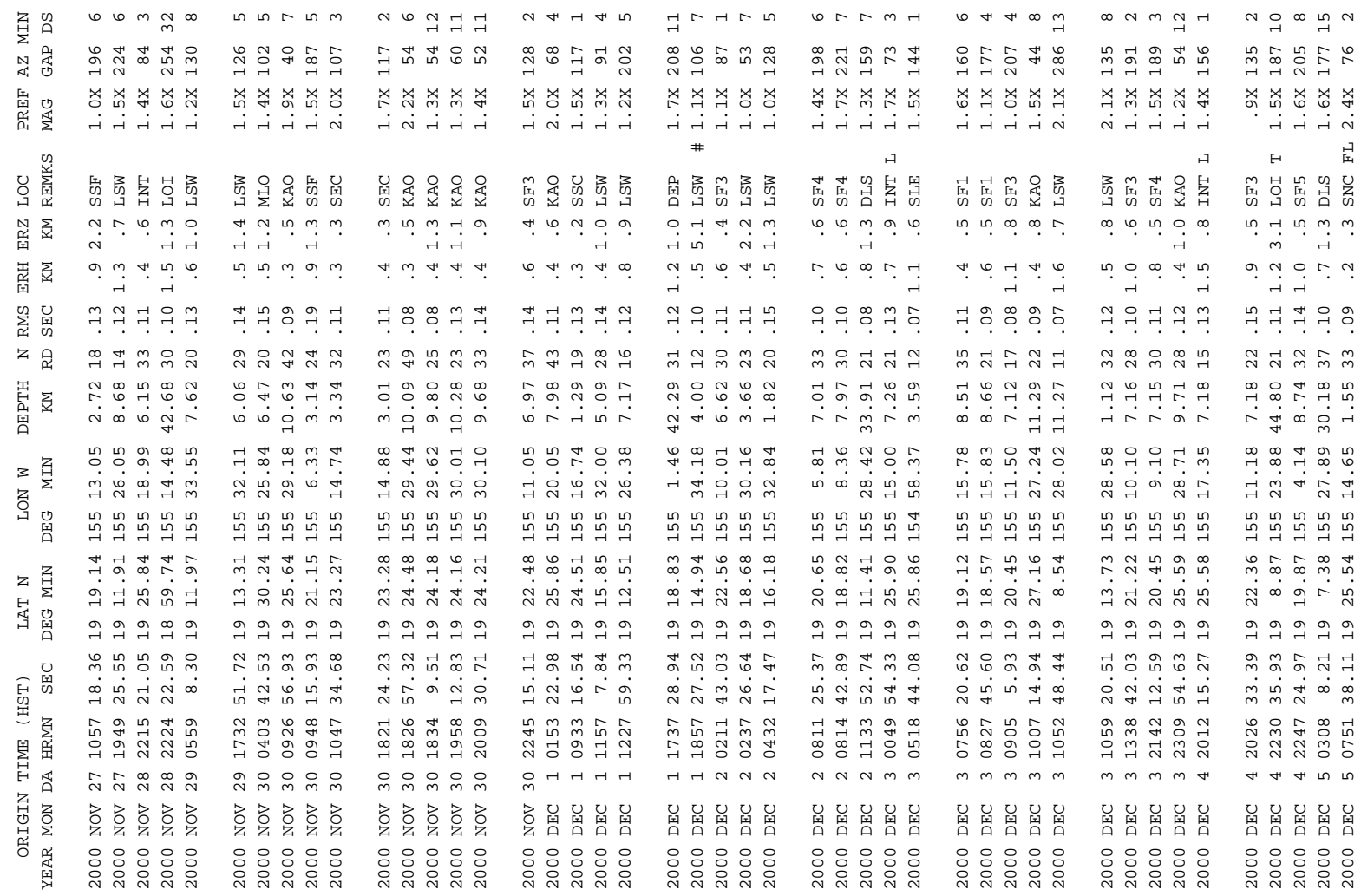

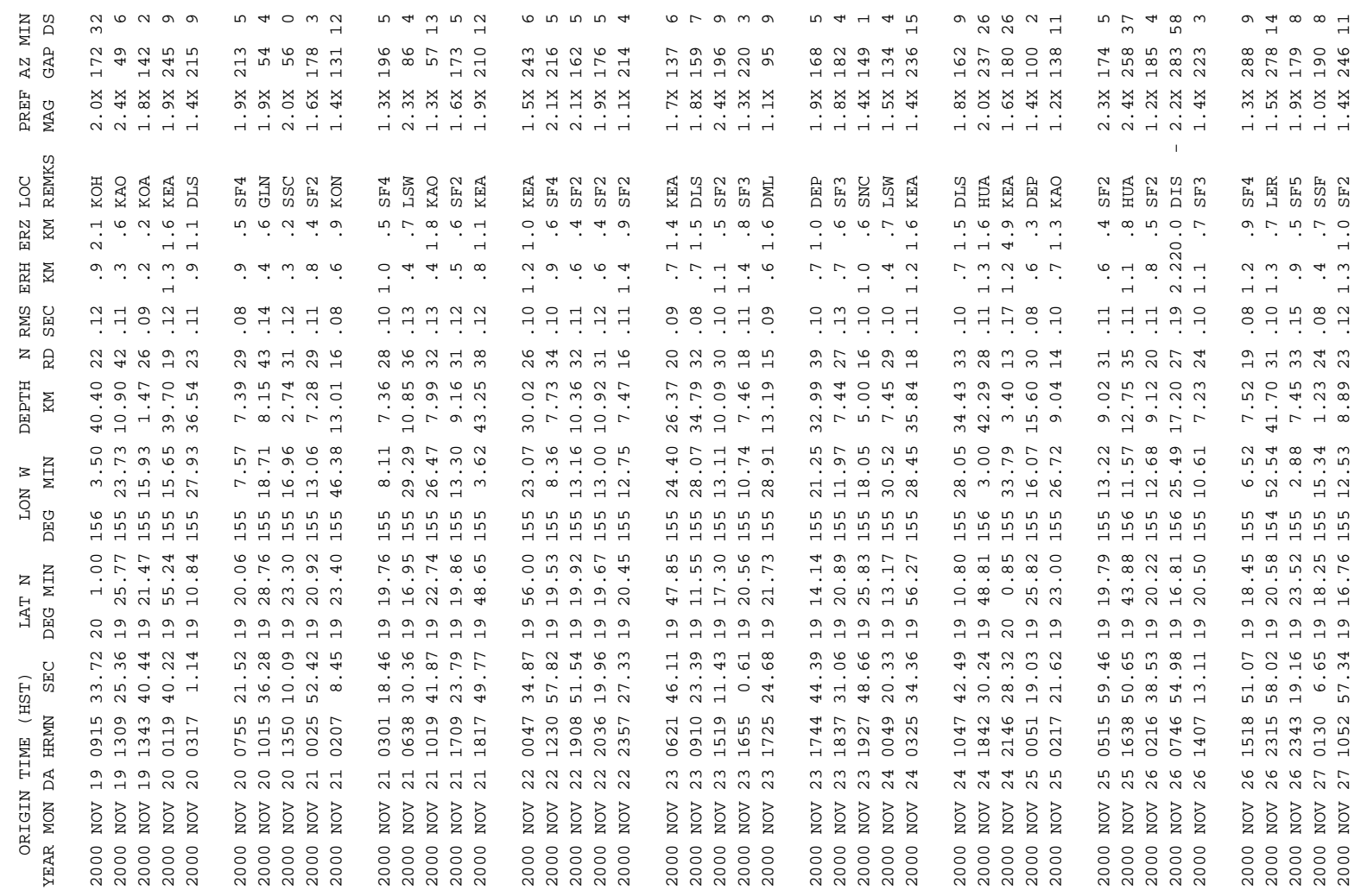




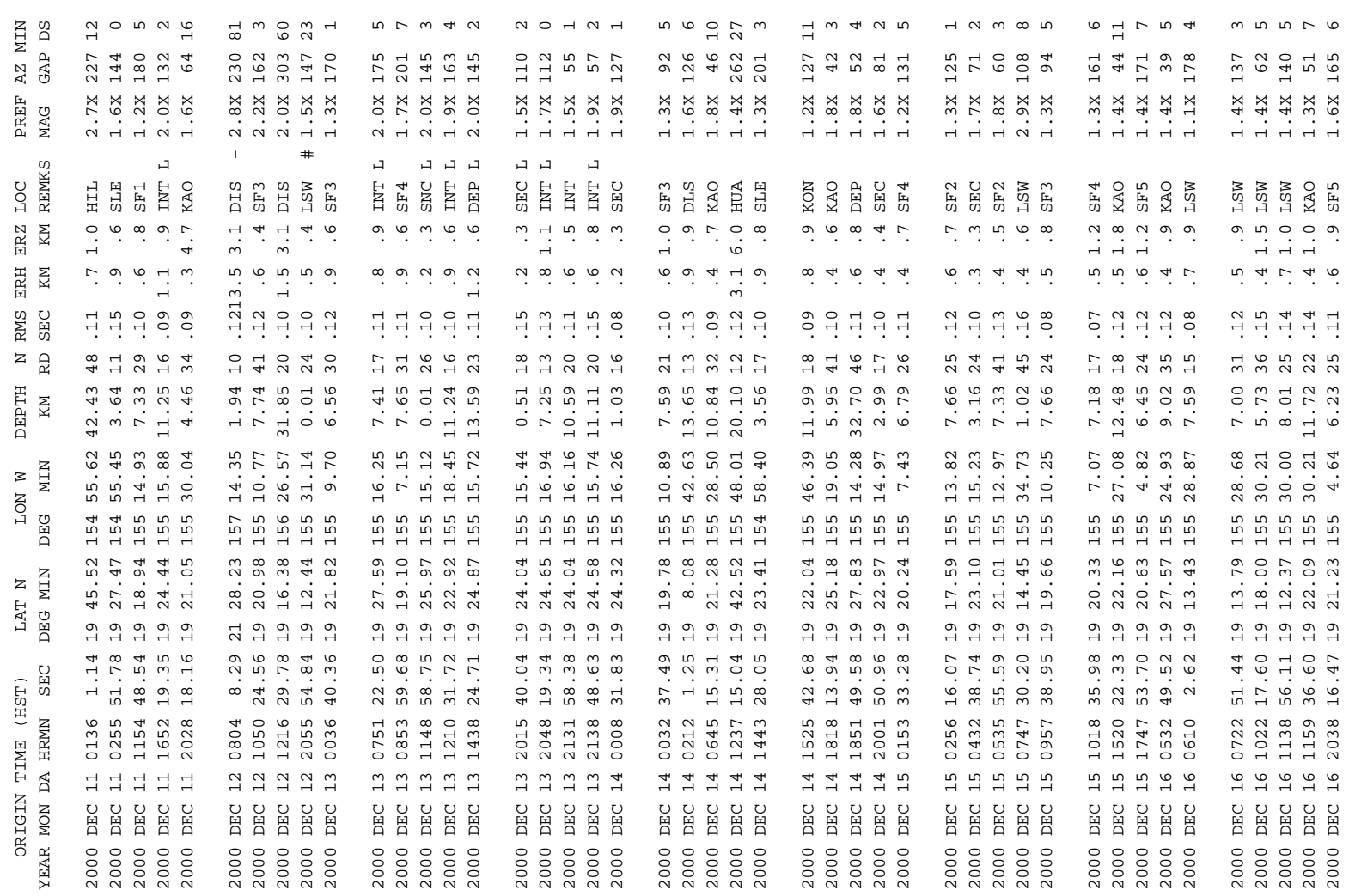

\begin{tabular}{|c|c|c|c|c|c|c|c|c|}
\hline 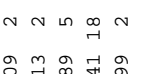 & 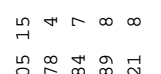 & $\begin{array}{l}\text { IN } \sim \infty \pi r \\
\because N 00 r\end{array}$ & 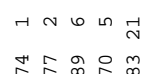 & 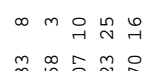 & 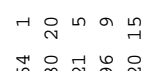 & 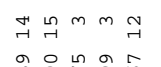 & 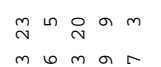 & \\
\hline 业 & 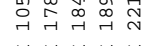 & $\vec{N} \underset{N}{N} \vec{N}$ & デト & 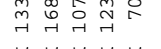 & $\stackrel{\sim}{\sim} \underset{N}{N} \underset{N}{N} \underset{\sim}{N}$ & 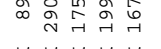 & 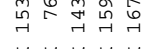 & 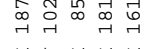 \\
\hline & : & 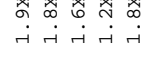 & 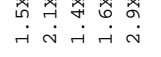 & 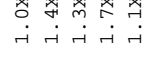 & 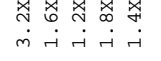 & 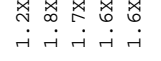 & 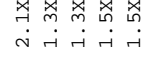 & 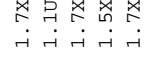 \\
\hline & & & & & & & & $\#$ \\
\hline & 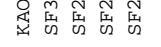 & 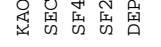 & 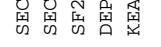 & 罢总舀总 & 番诖 & 俤要 & & E \\
\hline & & & m. ․ำ & $\infty$. & $r \cdot{ }_{i}^{m} \stackrel{r}{i}$ & 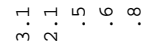 & ${ }^{\circ}$ & i \\
\hline & $\stackrel{\curvearrowleft}{?}$ & ช. ग & 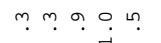 & $\stackrel{\infty}{.}$ & & ra & ?. ๑ ? & \\
\hline 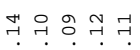 & 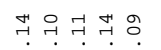 & $\stackrel{m}{7}: \overrightarrow{0}: \overrightarrow{7} 7 \overrightarrow{7}$ & 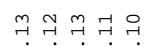 & 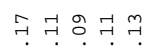 & 쿵웅욱ㄱ 국 & 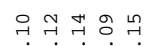 & 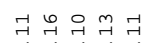 & 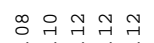 \\
\hline$\stackrel{\infty}{\sim}$ ने & $\stackrel{\infty}{\sim} \underset{\sim}{\sim} \stackrel{\sim}{\sim} \stackrel{\infty}{\sim} \underset{\sim}{\sim}$ & $\vec{\sigma} \mathfrak{N} \vec{m} \vec{F}$ & 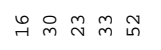 & $\underset{\sim}{m} \underset{\sim}{\infty} \stackrel{m}{m} \underset{\sim}{N}$ & $\sigma_{\sigma} \sigma \underset{\sim}{\sim} \approx \stackrel{n}{m}$ & 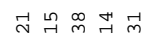 & $\ddot{m}$ & $\stackrel{\sim}{\sim} \underset{\sim}{\mathbb{N}} \mathbb{N} \underset{m}{N}$ \\
\hline $\begin{array}{l}\infty \\
\ddots \\
\vdots\end{array}$ & 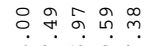 & 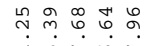 & 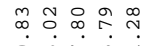 & ఇุ? & $\mathcal{F} \stackrel{\sim}{\sim} \stackrel{m}{\sim} m$ & $\operatorname{5n}$ & Ș. & 둥ํำ \\
\hline & $\infty_{\infty}^{\infty}$ & $\stackrel{-1}{6}$ & 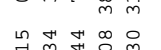 & 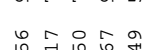 & 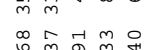 & 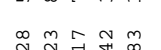 & 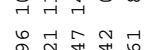 & \\
\hline অ্ & $\dot{x}$ & & 它 & 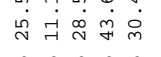 & & & & +6 \\
\hline 惢员 & 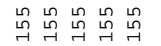 & 吕总总总出出 & 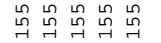 & 虽品品虽出 & 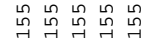 & 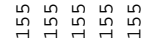 & 怘虽虽虽出 & 总号吕吕号 \\
\hline & 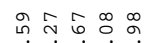 & 유 & 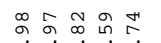 & $\vec{m}$ & , & $=0$ & ले जि & $\overbrace{}^{2}$ \\
\hline & $\dot{i} \dot{\sim} \stackrel{\infty}{\infty} \stackrel{\infty}{\rightarrow} \dot{\sim}$ & $\dot{\sim} \sim \stackrel{\infty}{\sim} \stackrel{\infty}{\sim} \stackrel{\dot{\sim}}{\sim}$ & ป⿻ & & & & & 80 \\
\hline$\theta \sigma$ & ને & ने નેને ને ને & 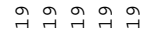 & ने & ने ने & ने & त) & न \\
\hline & mे & 돈ำ & 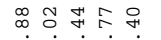 & & $\stackrel{\sim}{\sim}$ & $8=0$ & & \\
\hline & in & 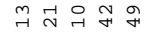 & 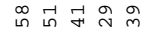 & $m \sim$ & $t^{\pi}$ & 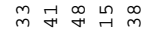 & & $\dot{\vec{y}} \dot{\leftrightarrow} \dot{+}$ \\
\hline 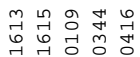 & 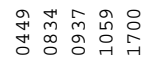 & 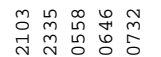 & 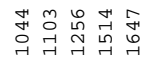 & 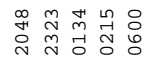 & & & & 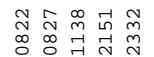 \\
\hline & & & $r$ & & & & & \\
\hline & & 嘼 & $\begin{array}{l}\text { 曾曾曾畕 } \\
\end{array}$ & a & 言 & 界 & & 曾曾曾我 \\
\hline & & & & & & & & \\
\hline
\end{tabular}




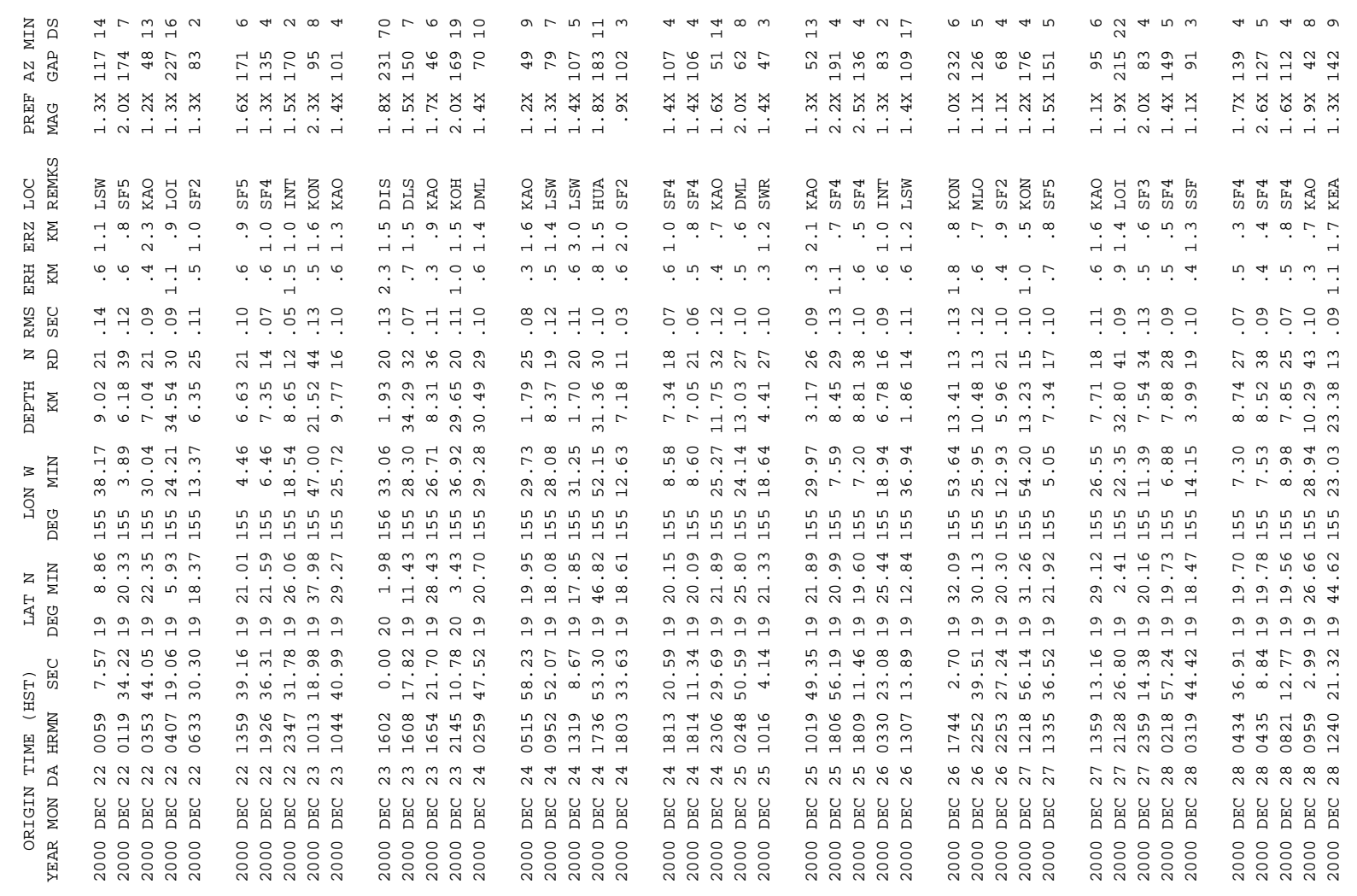

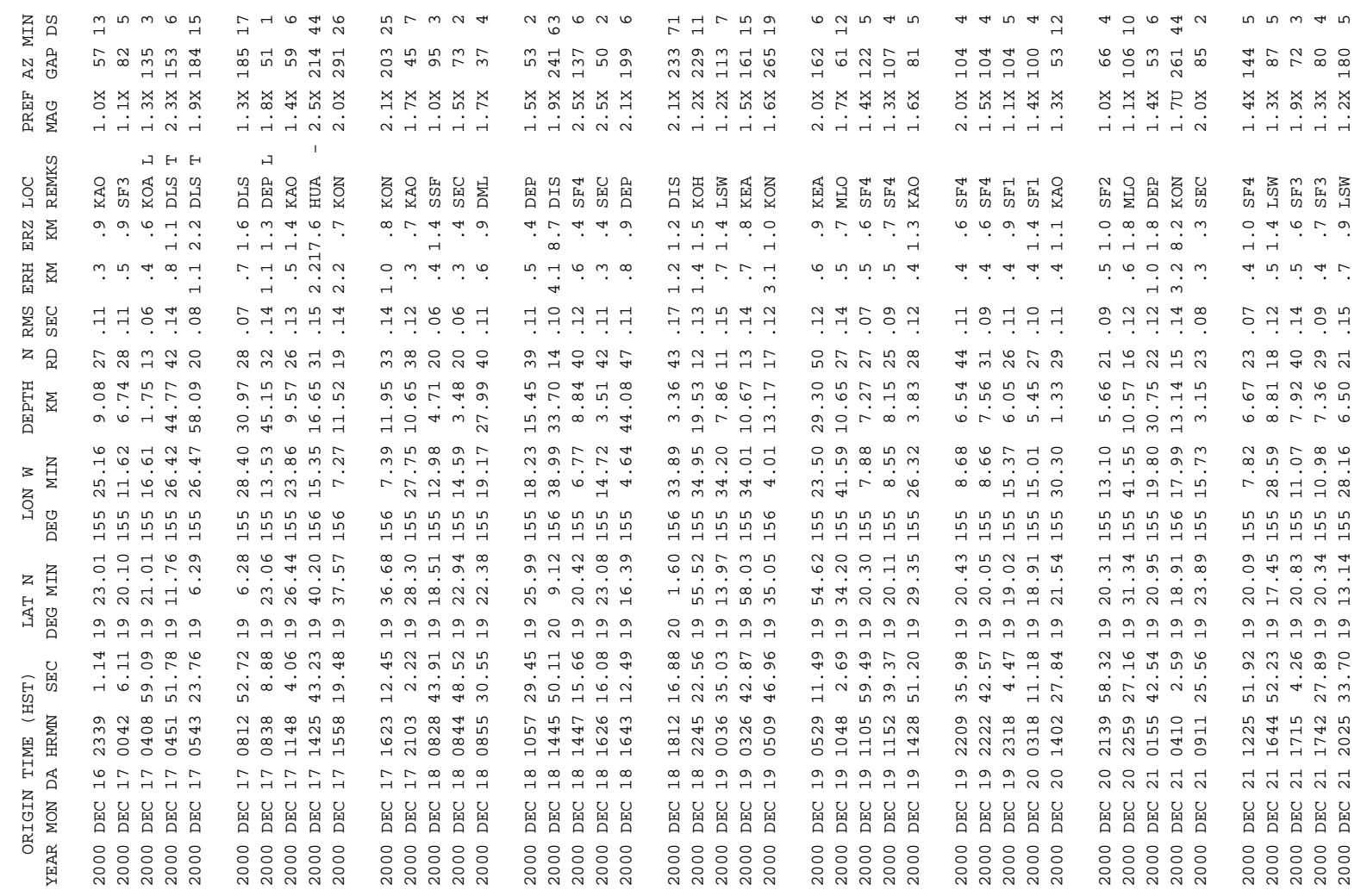




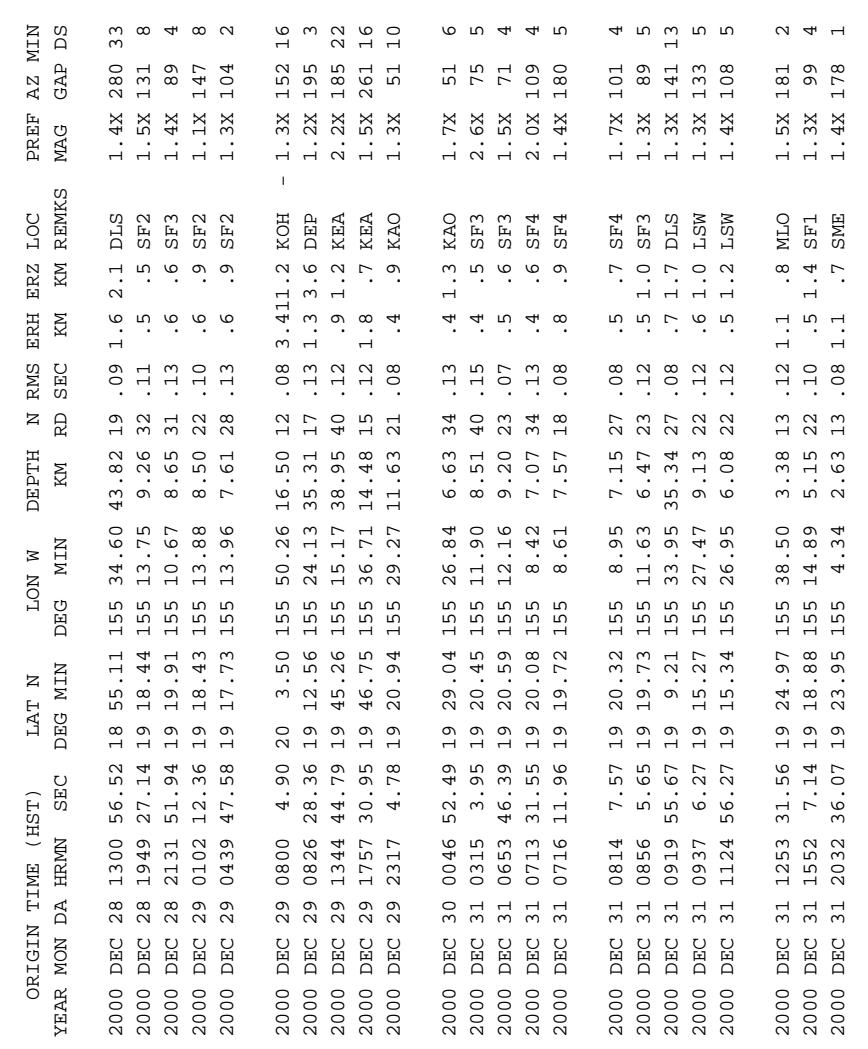


Table 5 .

ORIGIN TIME (HST) LAT $N$ LON W DEPTH N RMS ERH ERZ LOC PREF AZ MIN YEAR MON DA HRMN SEC DEG MIN DEG MIN KM RD SEC KM KM REMKS MAG GAP DS

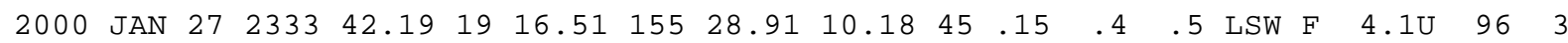
$\begin{array}{llllllllllllllllll}2000 & \mathrm{FEB} & 4 & 0053 & 16.39 & 19 & 16.35 & 155 & 28.09 & 10.56 & 34 & .13 & .3 & .4 & \text { LSW } & 3.0 \mathrm{X} & 96 & 4\end{array}$ $\begin{array}{llllllllllllllllllll}2000 & \text { FEB } & 11 & 1719 & 55.82 & 19 & 34.48 & 156 & 13.98 & 22.07 & 35 & .10 & 1.2 & 2.0 & \text { KON } & \text { F } & 3.3 \mathrm{X} & 220 & 24\end{array}$

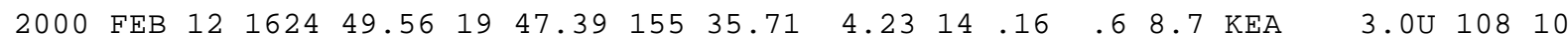
$\begin{array}{llllllllllllllllll}2000 & \text { FEB } & 13 & 0202 & 54.53 & 20 & 9.05 & 155 & 55.74 & 31.76 & 48 & .11 & .6 & .8 & \mathrm{KOH} & 3.3 \mathrm{X} & 134 & 5\end{array}$

$\begin{array}{lllllllllllllllllll}2000 & F E B & 17 & 1418 & 42.32 & 19 & 20.58 & 155 & 16.14 & 35.66 & 32 & .10 & .7 & 1.2 & \text { DEP } & \text { F } & 3.4 X & 148 & 2\end{array}$ $\begin{array}{lllllllllllllllllll}2000 & \text { FEB } & 17 & 1419 & 28.77 & 19 & 20.22 & 155 & 16.76 & 35.29 & 37 & .11 & .7 & .9 & \text { DEP } & \text { F } & 4.5 \mathrm{U} & 111 & 1\end{array}$

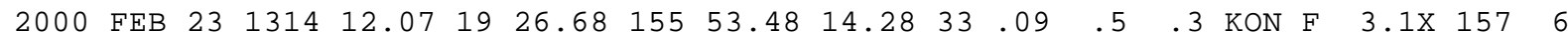

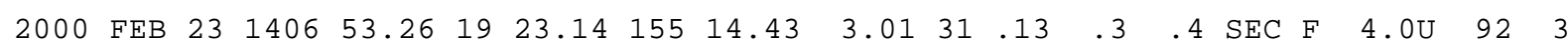

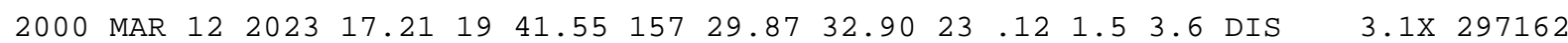

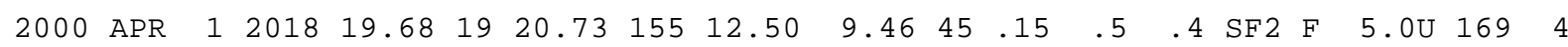

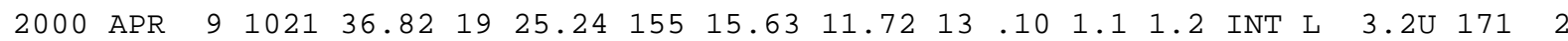

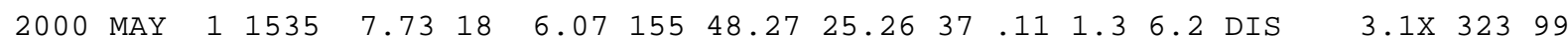
$\begin{array}{lllllllllllllllll}2000 & \text { MAY } 4 & 1743 & 36.34 & 19 & 11.85 & 155 & 38.90 & 7.32 & 42 & .16 & .5 & .8 & \text { LSW } & 3.1 X & 106 & 6\end{array}$

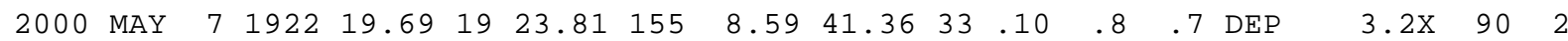

$\begin{array}{llllllllllllllllllll}2000 & \text { MAY } & 15 & 2255 & 19.38 & 19 & 45.92 & 156 & 39.58 & 31.42 & 29 & .10 & 1.1 & 2.3 & \text { DIS } & 3.4 \mathrm{X} & 261 & 68\end{array}$

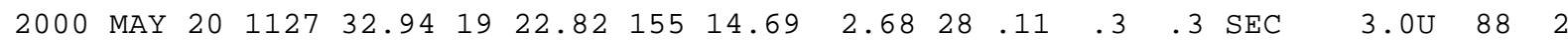

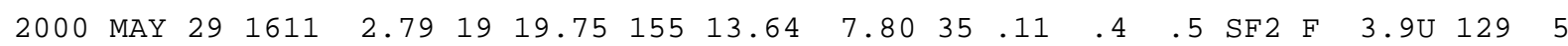

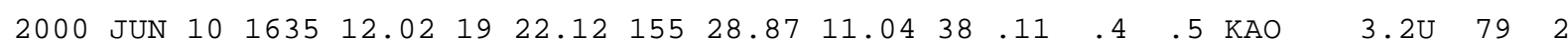

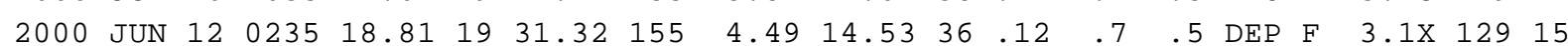

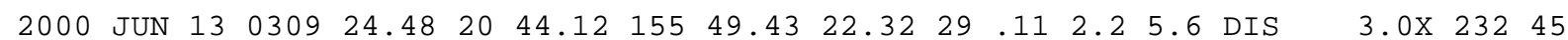

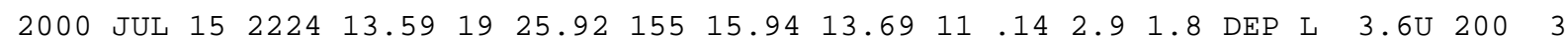

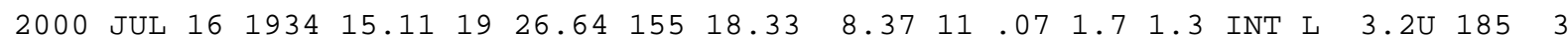

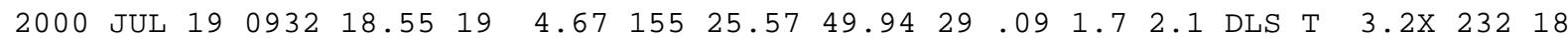

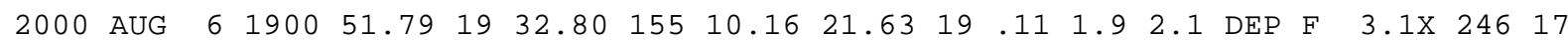

$\begin{array}{llllllllllllllllllll}2000 & \text { OCT } & 13 & 2153 & 42.28 & 20 & 17.84 & 155 & 26.14 & 38.81 & 60 & .10 & .7 & 1.5 & \text { KEA } & \text { F } & 3.9 \times & 205 & 41\end{array}$

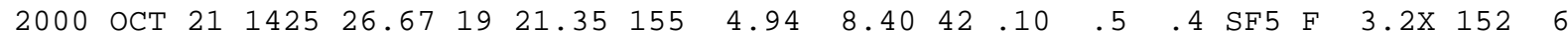

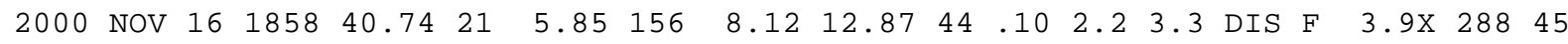
$\begin{array}{lllllllllllllllllll}2000 & \mathrm{DEC} & 8 & 1003 & 11.20 & 19 & 19.58 & 155 & 17.68 & 35.12 & 49 & .11 & .6 & .7 & \mathrm{DEP} & \mathrm{F} & 3.2 \mathrm{X} & 154 & 1\end{array}$ 